\title{
Integration of a personalized health care model into health systems and policies in Europe
}

Citation for published version (APA):

Cesuroglu - de Langen, T. (2016). Integration of a personalized health care model into health systems and policies in Europe. [Doctoral Thesis, Maastricht University]. Maastricht University. https://doi.org/10.26481/dis.20160218tc

Document status and date:

Published: 01/01/2016

DOI:

$10.26481 /$ dis.20160218tc

Document Version:

Publisher's PDF, also known as Version of record

\section{Please check the document version of this publication:}

- A submitted manuscript is the version of the article upon submission and before peer-review. There can be important differences between the submitted version and the official published version of record.

People interested in the research are advised to contact the author for the final version of the publication, or visit the DOI to the publisher's website.

- The final author version and the galley proof are versions of the publication after peer review.

- The final published version features the final layout of the paper including the volume, issue and page numbers.

Link to publication

\footnotetext{
General rights rights.

- You may freely distribute the URL identifying the publication in the public portal. please follow below link for the End User Agreement:

www.umlib.nl/taverne-license

Take down policy

If you believe that this document breaches copyright please contact us at:

repository@maastrichtuniversity.nl

providing details and we will investigate your claim.
}

Copyright and moral rights for the publications made accessible in the public portal are retained by the authors and/or other copyright owners and it is a condition of accessing publications that users recognise and abide by the legal requirements associated with these

- Users may download and print one copy of any publication from the public portal for the purpose of private study or research.

- You may not further distribute the material or use it for any profit-making activity or commercial gain

If the publication is distributed under the terms of Article $25 \mathrm{fa}$ of the Dutch Copyright Act, indicated by the "Taverne" license above, 


\section{Integration of a \\ Personalized Health Care Model \\ into \\ Health Systems and Policies in Europe}

Tomris Cesuroglu 
Integration of a Personalized Health Care M odel into Health Systems and Policies in Europe

Tomris Cesuroglu

ISBN: 978-94-6233-221-8

Cover: Serenad Yılmaz, Veysel Aybakar

Printing: Gildeprint, Enschede

(c) copyright Tomris Cesuroglu, Maastricht, the Netherlands, 2016.

All rights reserved. No part of this publication may be reproduced or distributed in any form or by any means, or stored in a database or retrieval system, without the prior written permission of the copyright owner.

tomris.cesuroglu@gmail.com 


\title{
Integration of a \\ Personalized Health Care Model \\ into \\ Health Systems and Policies in Europe
}

\author{
DISSERTATION \\ to obtain the degree of Doctor at M aastricht University, \\ on the authority of the Rector Magnificus, Prof. dr. L.L.G. Soete \\ in accordance with the decision of the Board of Deans, \\ to be defended in public \\ on Thursday 18 February 2016, at 16:00 hours
}

by

Tomris Cesuroglu 


\section{Supervisor}

Prof. Dr. Frans J. M. Feron

\section{Co-supervisors}

Dr. Anja Krumeich

Dr. Elena V. Syurina

\section{Assessment Committee}

Prof. Dr. Nanne de Vries (chair)

Dr. Heidi C. Howard (Uppsala University, Sweden)

Prof. Dr. David Townend

Dr. Petra Verdonk (Vrije Universiteit Amsterdam)

The project team for this dissertation also included Serdar Savaş as an external member.

The research presented in this dissertation was conducted at the School for Public Health and Primary Care: CAPHRI, Department of Social M edicine, Faculty of Health, M edicine, and Life Sciences, M aastricht University. CAPHRI participates in the Netherlands School of Primary Care Research CaRe. 
Dedicated to my husband M arco, and our children Yunus, M ina and Alanur 



\section{CONIENIS}

Chapter 1. Introduction

PART I - Where to integrate? $\quad 31$

Chapter 2. A framework for public health and health systems interrelations: $\quad 33$ Health Globe

Chapter 3. Looking at the U.S. health system from European perspective

PART II - What to integrate?

Chapter 4. Debating the definition of personalized approaches: Content analysis of practices

Chapter 5. A perspective on personalized health care

Chapter 6 . Introducing a preventive personalized health care model: Gentest

Chapter 7. A systematic approach to assess integration of a preventive personalized health care model into health systems

Chapter 8. General discussion

Appendix

Executive summary | Samenvatting | Özet 305

Valorization

Acknowledgements | Dankwoord | Teşekkürler 329

CV \& Publications 



\section{The list of frequently used abbreviations}

EU: European Union

GDP: Gross domestic product

GP: General practitioners/ Family physicians (physicians who provide medical services within primary care)

HFA: Health for All

HiAP: Health in All Policy

ICT: Information and communication technologies

NCD: Noncommunicable disease

NGO: Non-governmental organization

NHS: National Health Services

OECD: Organisation for Economic Co-operation and Development

PAHO: Pan American Health Organization

PM/PHC: Personalized medicine and personalized health care

U.S.: United States

UK: United Kingdom

UN: United Nations

USA: United States of America

USD: United States Dollars

WHO: World Health Organization 
Our true mentor in life is science.

M ustafa Kemal Atatürk 
Chapter 1

Introduction 


\section{Contents}

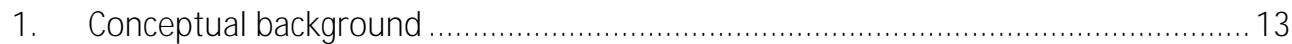

1.1. Burden of chronic diseases and its impact on health systems........................ 13

1.2. A brief look at the underlying causes of complex diseases............................ 17

2. A preventive practice model for personalized health care: Gentest ...................... 21

3. The aim and structure of the dissertation ....................................................... 23

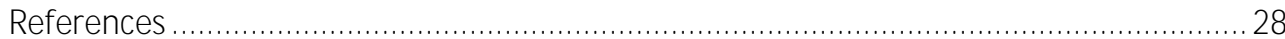


Every day, we read about new scientific discoveries and technological advancements in the newspapers. The news has been very promising for medicine and health, in particular in reference to 'genetics' in the past 15-20 years: "Diabetes gene is found!", "Heart attack gene is found!", "Cancer gene is found", "We'll be able to prevent diabetes in 10 years!"... However, we don't see these 'genes' actually at work in health services. Somehow, they don't complete their journey from the laboratory to the society.

This PhD research deals with the integration of personalized health care applications into health services. The setting, i.e. health services, into which genetic based innovations should be integrated, is a part of the broader context of health systems. Therefore, the first part of the dissertation explores health systems and its interrelations with 'public health'. The second part introduces a preventive personalized health care model called Gentest, within the wider context of the field of personalized medicine and health care. This practice model is used as an example in this dissertation to explore the issues related to integration effectively. The third part investigates how this practice model can be integrated into primary care services within European health systems.

In this introductory chapter, first the conceptual background that signifies the societal need for prevention of chronic complex diseases and an approach towards prevention is explained. Then the practice model is briefly introduced. Finally, the aim and structure of the dissertation is summarized.

\section{Conceptual background}

\subsection{Burden of chronic diseases and its impact on health systems}

As a result of the epidemiological and demographic transition, life expectancy has been rapidly increasing since the $20^{\text {th }}$ century. In the $21^{\text {st }}$ century, the main burden of disease is caused by chronic complex diseases, which stem from the complex interaction of human genome with lifestyle and environmental factors. Cardiovascular and cerebrovascular diseases, cancers and diabetes are among major chronic complex diseases, which account for approximately two third of all deaths in the world. Thus, $21^{\text {st }}$ century brings us to an aged population living with chronic conditions, creating a huge burden on health systems and society.

\section{Definition}

The terms used to refer to this group of diseases vary, depending on the perspective of the disciplines or type of stakeholders. Most common definition, used mostly in health care sector and among health professionals, is 'chronic diseases', referring to their long duration and slow progression. Another term mostly used by organizations and professionals that address the health problems on the population level, such as international and intergovernmental agencies, is 'Noncommunicable diseases' (NCDs). NCDs constitute 
one of the three main clusters of diseases and conditions that lead to health loss in Global Burden of Disease Project [1]. NCDs largely overlap with chronic diseases and are often used synonymously. 'Complex diseases' is another term often used synonymous to 'chronic diseases', but emphasizes the underlying cause of the conditions in question, i.e. complex interaction of the genetic structure of the individuals with lifestyle and environmental factors. This term is more commonly seen in scientific and technological contexts. 'Lifestyle diseases' is sometimes used in media when emphasizing the importance of lifestyle factors in development of chronic diseases. Throughout this dissertation, these terms are used to refer to chronic diseases, depending on the context and purpose.

\section{Total deaths - Global, 2012}

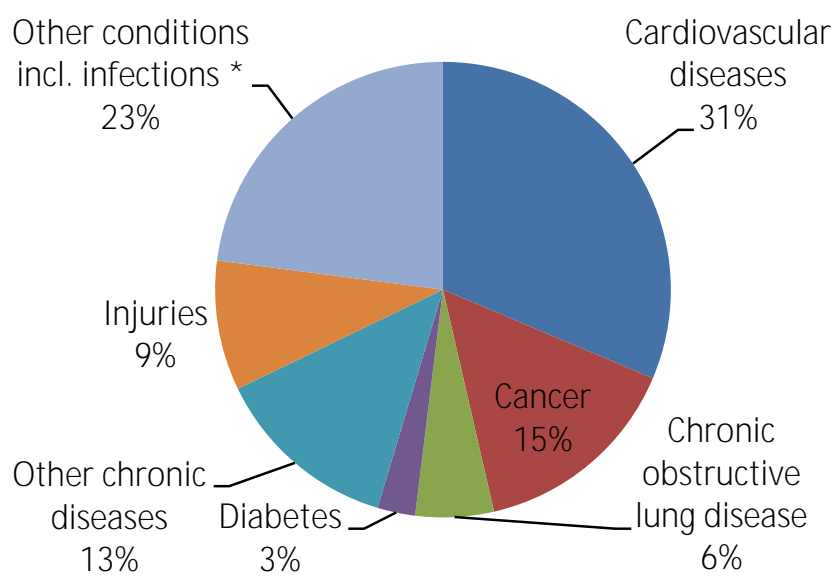

\section{Figure 1: Total deaths by causes}

* Other conditions include communicable, maternal, perinatal and nutritional conditions. Data source: WHO, 2014 [2]

\section{Extent of the problem globally and in Europe}

Chronic diseases are the leading cause of mortality globally, responsible for almost $70 \%$ of the deaths in the world. $40 \%$ of them are premature deaths under the age of 70 years [3]. If not intervened effectively, deaths from chronic diseases will increase by $15 \%$ between 2010 and 2020 [4].

The burden of chronic diseases has been rapidly increasing in all parts of the world. In most countries, they cause $50 \%$ or more of all healthy life-years lost. In richer countries such as North America and most of Europe, this percentage is greater than $80 \%$ [1].

The situation regarding these conditions in Europe is serious due to two reasons. First, despite progress made in reducing mortality from ischemic heart disease and stroke in the 
past 20 years, overall burden of chronic diseases continues to increase [5]. As of 2010, in European Union (EU) and European Free Trade Association (EFTA) countries, chronic diseases are responsible for $90 \%$ of all deaths and $86 \%$ of the disease burden [6].

Secondly, population in Europe is aging very rapidly. The population above the age of 65 , as well as the proportion of aged people to working age (15-64) will double from 2010 to $2060[7,8]$. As the prevalence of chronic diseases increases with age, the problem of caring for elderly with chronic diseases will lead to huge social and economic burden on the European countries. Nevertheless, one must note that chronic diseases are not only the problem of the elderly anymore. Rising numbers of young and middle-aged people have some sort of chronic health problem, leading to premature mortality, as well as morbidity in the working ages [9].

The economic impact of chronic diseases is serious. They constitute huge costs for individuals, families, businesses, governments and health systems, cause billions of euros in losses of the national incomes each year. And the problem is predicted to increase with time. The cost of chronic diseases will double from 2010 to 2030. The anticipated total economic toll of cardiovascular diseases, cancer, chronic lung diseases and diabetes in 2010-2030 globally is more than 30 trillion United States Dollars (USD), representing $48 \%$ of global gross domestic product (GDP) in 2010 and pushing millions of people below the poverty line [10]. Moreover, chronic diseases have a negative effect on the labor market and formation of the human capital [11]. Each $10 \%$ rise in prevalence of chronic diseases is associated with $0,5 \%$ lowering of the rates of annual economic growth [4].

Currently, $70-80 \%$ of all health care budgets, i.e. 700 billion Euro per year, are spent on chronic diseases in EU [12]. The health budgets (in health systems with national health services) and the social insurance funds (in health systems with social health insurance models), as well as the budgets related to chronic care of elderly, will face great difficulties in coping with the financial burden caused by aging populations living with chronic conditions.

\section{Recognition of the problem and development of strategies}

The ever increasing burden of chronic diseases is recognized widely around the world, calling for development and adoption of effective intervention methods for their prevention and control.

A significant step has been the United Nations (UN) High Level Meeting on NCDs, held on 19-20 September 2011. It focused on the four main chronic diseases: cardiovascular disease, cancer, chronic lung diseases and diabetes. This was only the second time in the history of UN General Assembly that a high-level meeting was dedicated to a health issue (the first time was on HIV/AIDS in 2001). As a result of the meeting, world leaders adopted a political declaration, stating that NCDs reached epidemic proportions globally and there 
is an urgent need for greater measures to prevent and control them on global, regional and national levels [13]. Following this, World Health Organization (WHO) developed the Global NCD Action Plan for 2013-2020, which was endorsed by the World Health Assembly in May 2013, together with the global target to reduce deaths under 70 (premature deaths) from NCD's by $25 \%$ by 2025 [14].

WHO Europe had already addressed the issue with 'Gaining Health: the European Strategy for the Prevention and Control of Communicable Diseases' in 2006 [15]. The significance of current and upcoming burden of chronic diseases has also been recognized on the EU level, leading to the first EU Summit on Chronic Diseases in Brussels on 3-4 April 2014 [12].

The above mentioned international/ intergovernmental efforts and many others have been highlighting the importance of prevention as a key aspect of all strategies tackling with the burden of chronic diseases. In addition, management of the diseases and caring for the people living with chronic conditions is becoming a huge problem all around the world, in particular in Europe due to its rapidly aging population.

The question is whether health systems are sufficiently adapted to deal with the burden of chronic diseases as they were designed for the previous century, when communicable diseases accounted for the biggest share of the disease burden. Unlike communicable diseases, chronic complex diseases have multiple causative factors and occur concurrently rather than in isolation (multi-morbidity). To respond to the increasing burden of chronic diseases effectively, the reductionist paradigm of the previous century, i.e. 'single cause -> single disease $->$ single treatment', needs to be renewed with new visions and frameworks, such as a system thinking paradigm [16] and application of 'comprehensive' approaches that address multiple determinants of health. The question therefore is whether current health systems can effectively address them and whether they are proactive, health oriented (not disease), and preventive enough to provide adequate chronic care, and whether they have the right focus on the whole spectrum of health care (not only medicine).

Innovation is essential to equip health systems with new practices in order to respond to the growing burden of chronic diseases [17]. In an era where health systems are under serious pressure of the increasing costs and demands for increasing quality, they must innovate to keep functioning [18]. National Health Services (NHS) of United Kingdom (UK) has put it as: “... simply doing more of what we have always done is no longer an option. We need to do things differently. We need to radically transform the way we deliver services. Innovation is the way - the only way - we can meet these challenges..." [19]. The urgent need for innovation has also been recognized on the EU level and addressed within the European Innovation Partnership on Active and Healthy Ageing [20]. This partnership developed strategies in three areas: prevention and health promotion, care and cure, and active and independent living of elderly people. 


\section{Scope of the dissertation in terms of chronic diseases}

UN High Level Meeting on NCDs and the Global NCD Action Plan 2013-2020 focused on four main chronic diseases: cardiovascular diseases, diabetes, cancer and chronic obstructive lung diseases $[13,14]$. They are responsible for $80 \%$ of the deaths from chronic diseases, and $55 \%$ of all deaths globally (Figure 1). These conditions mostly have an adult onset and share common behavioral risk factors: tobacco use, unhealthy diet, physical inactivity and harmful use of alcohol. Actually, chronic diseases as a group is very broad and also includes issues such as mental health disorders, renal diseases, neurodegenerative conditions, musculoskeletal disorders and many others. They are associated with the risk factors of the above mentioned four major chronic diseases, but also have other specific risk factors. Since designing strategies to target them all would be unwieldy, the UN High Level Meeting on NCDs and the Global NCD Action Plan 2013-2020 have identified its focus as these four major ones. Due to similar concerns, it was decided to limit the scope of this dissertation to major chronic diseases of the adult onset, which share common underlying lifestyle factors. Examples of such conditions are: cardiovascular diseases, diabetes, cancer, chronic lung diseases and osteoporosis. It is of note that improved lifestyle will have a positive effect on not only these diseases, but also several other conditions, as well as slowing down the age related decline in physical and mental functions.

\subsection{A brief look at the underlying causes of complex diseases}

In order to develop strategies to tackle the problem of chronic diseases effectively and develop innovative solutions, we first need to understand how they are developing.

\section{Determinants of health and role of genetic structure in development of complex diseases}

There have been a number of models attempting to explain the factors that influence health and development of diseases [21-27]. What they all have in common is that health is influenced by multiple factors that surround individuals, although the specific clusters, layers and perspectives may vary.

Based on the classical approach to 'determinants of health', health is affected by biological factors including genome, lifestyle and environmental factors, health systems in the broader and health services in the narrower context, and socioeconomic dimensions (Figure 2).

Chronic diseases are caused by the interaction of genetic structure and lifestyle and environmental factors. However, their genetic basis is different from, for example, the underlying abnormalities in genetic diseases. In order to understand the genetic basis of chronic diseases, we may visualize the diseases in a threshold model as shown in Figure 3. Here, diseases are grouped into four major areas, according to the causative factors. 


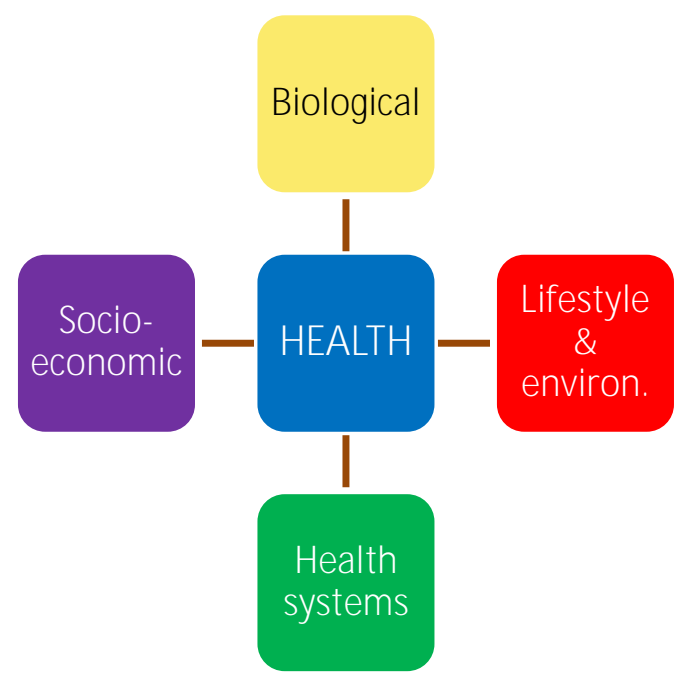

Figure 2: Determinants of health

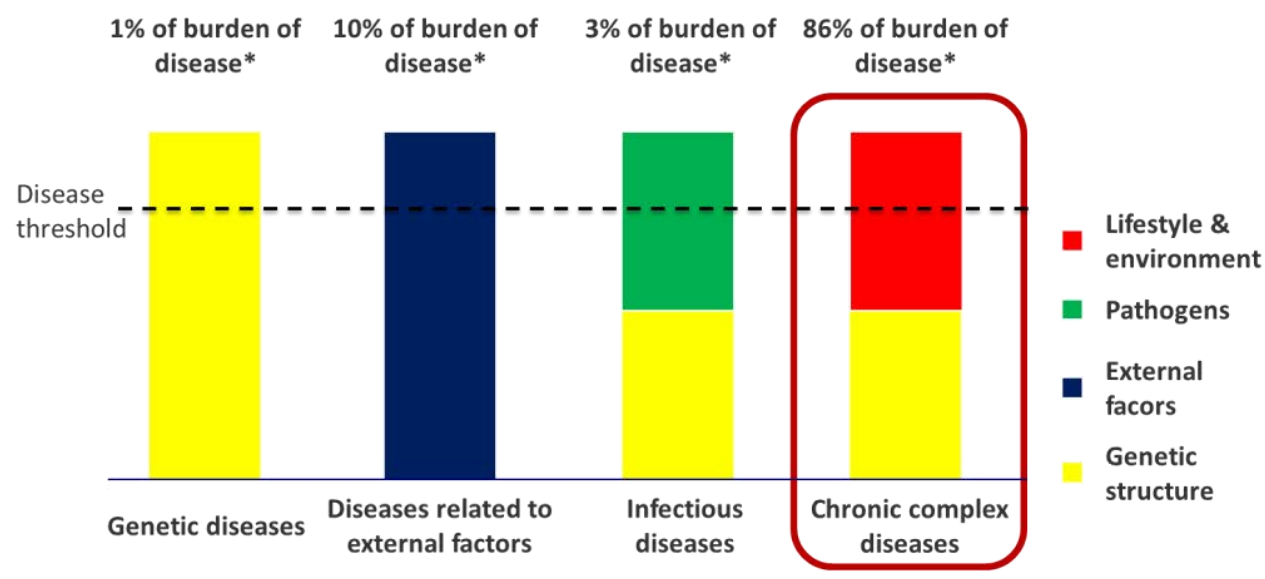

Figure 3: Interaction of genes with lifestyle and environment from a disease threshold perspective

* Estimates for burden of disease in EU and EFTA Countries based on data provided by the Global Burden of Disease Study 2010[6] Source: Modified from Cesuroglu et al., 2009 [28]

The first group is genetic diseases, which are caused by abnormalities in the genetic structure of individuals. Down syndrome, cystic fibrosis, phenylketonuria are disorders caused by deleterious changes in genetic structure. They constitute a small fraction of burden of disease. External and lifestyle and environmental factors play little or no role in most of these diseases (first column in Figure 3). 
The second group is caused by external factors and includes injuries, including traffic accidents, violence, poisoning, etc. Genetic structure doesn't have a prominent role in their development. They constitute around $10 \%$ of the burden of disease in EU and EFTA countries (second column in Figure 3).

The third group includes infectious diseases that were believed to be only caused by pathogens. However, growing body of research indicates that host genomic factors also play an important role in manifestation of infectious diseases (third column in Figure 3). The burden of these conditions used to be very high over the course of the history of mankind; however it reduced drastically throughout the last century, in particular in high-income countries. Although infectious diseases still create a significant burden in certain parts of the world, such as Africa, globally their proportion is reducing.

The fourth group consists of disorders where genetic structure creates a predisposition to the disease, but it is not enough to make one sick. They develop when other factors, mainly unfavorable lifestyle factors, as well as the environment, add on the genetic predisposition (fourth column in Figure 3). These are chronic (or complex) diseases that develop due to the interaction between these genetic and other components. The environment here refers to chronic exposure, whereas external factors in the previous group refer to short term events with substantial effect.

\section{Development of chronic complex diseases}

In order to develop intervention models to prevent chronic diseases, we need to understand when and how to intervene. For this purpose, we take the column of chronic complex diseases in Figure 3 (fourth column), and put it in a life course perspective in Figure 4. In this hypothetical model, the left end of the figure is conception of an individual and the right end is death. The genetic structure (yellow part in the bottom) doesn't change in his/her life course. However, negative lifestyle and environmental conditions add on top of the genetic predisposition in time. For example, maternal smoking behavior, not being breastfed long enough, starting eating junk foods early in life, becoming physically inactive in school years, starting to smoke in teenage years, continuing unhealthy nutrition in early adulthood, living in polluted urban areas, continuing sedentary life, being exposed to work stress, financial problems and marital problems in later adulthood, etc. all add on (Phase I - Disease development).

This continues until the individual reaches the 'early signs' threshold and enters 'Phase II Early signs of disease'. Here, for example, the blood pressure, fasting plasma glucose and LDL cholesterol become higher than normal. The individual is not aware of them; they can only be detected by body measurements, laboratory tests, etc. These are also regarded as 'risk factors'. 
If the individual continues the negative lifestyle and keeps being exposed to negative environmental factors, he crosses the disease threshold and develops, for example, a heart attack. This is 'Phase III - Established disease'.

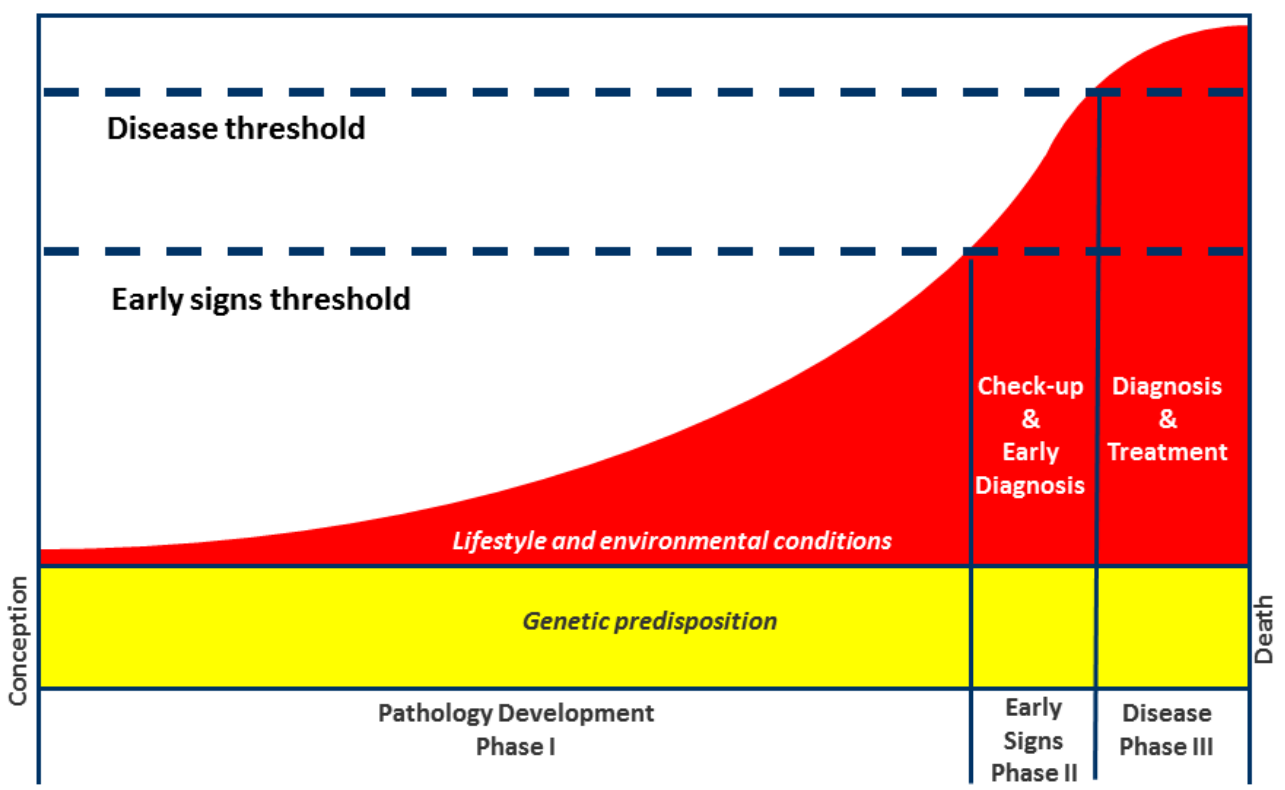

Figure 4: Development of chronic diseases in a time perspective - Disease development curve

Source: This model was developed by Serdar Savas at GENAR Institute for Public Health and Genomics Research, Ankara, Turkey, in 2008, inspired by the disease progression curve of Snyderman [29] to a certain extent.

Until recent past, overwhelming focus of health services has been on diagnosing and curing the diseases after the disease develop, i.e. 'late curative paradigm'. However, in the last decades, we have been witnessing a shift from 'late curative paradigm' towards 'early diagnosis'. Importance of check-up and early diagnosis has being more and more recognized because of possibilities to delay the onset of many diseases with preventive use of medications (such as aspirin, hypertension drugs and statins for cardiovascular disease prevention), as well as targeted diet and exercise. Thus, if the disease is caught and diagnosed in an early stage (Phase II) serious complications can be prevented or delayed. For example, many people with type 2 diabetes live with it undiagnosed, causing serious damage on various organs in the body. Thus, in Phase II, the main preventive intervention is screening for the disease.

The interventions on the earlier phases of disease development are more effective for its prevention, or minimization of the complications. Conversely, as the pathology development progresses, the costs related to detection, management and treatment of the dis- 
ease and/or risk factors are increasing [29]. Thus, the effectiveness of the interventions increases and costs decreases as we shift our focus to the left in Figure 4.

The shift from late curative paradigm to early diagnosis and medical management of the early signs of the disease has made significant contribution to reducing the mortality in industrialized countries for some diseases, such as cardiovascular diseases [30]. Nevertheless, this cannot slow down the increasing burden of many others, such as diabetes, chronic obstructive lung disease, and cancers. Thus, the current shift is not enough. There is still a large area, on the left side of the graphic: Phase I - Pathology development. This is the most crucial time period because the pathology actually develops in the long years before the early signs can be detected, starting from conception. However, this phase is currently not covered effectively by health interventions.

\section{Opportunities brought by personalized medicine and health care}

Currently, the best known prevention for chronic diseases is adopting a healthy lifestyle. However, this cannot be not achieved in many places of the world. Effective intervention models for lifestyle change to prevent these diseases are urgently needed. At this point, personalized medicine and health care can offer significant opportunities.

Throughout the whole spectrum of health care, there has been a gradual shift from a 'one size fits all' strategy towards personalized interventions, which can be applicable for health promotion, disease prevention, diagnosis, treatment, and rehabilitation. This shift is driven by the general societal trend towards personalization, as well as the rapid scientific and technological advancements in genome-based science/technologies and information/communication technologies [31]. This future vision, in the broadest sense, can be called as personalized medicine and health care and offers great opportunities to tackle the burden of chronic diseases.

\section{A preventive practice model for personalized health care: Gentest}

As an endeavor to face the challenge of chronic diseases summarized in previous section, GENAR Institute for Public Health and Genomics Research in Turkey developed a health service model called Gentest.

Gentest is a comprehensive and integrative model for prevention of common chronic diseases in primary care settings. It utilizes individuals' personal health information (clinical information), detailed lifestyle analysis, body composition, genotype, and other biomarkers (such as LDL cholesterol, fasting plasma glucose, etc.) in order to prevent development and progression of chronic diseases in a targeted way. Based on the results of the aforementioned components, risk assessments are made for the major chronic diseases including heart attack, stroke, type 2 diabetes, cancers (lung, breast, prostate, colon, and 
gastric cancers), and osteoporosis. Using all of the assessments, an optimum lifestyle plan, including personal menu plans and food exchange lists, exercise plans, smoking cessation recommendations, and a medical follow-up plan are drawn. The model is developed to be provided via health care professionals. Its mission is changing the behavior and managing the health of individuals according to their health needs and priorities. The conceptual framework of the practice model is presented in Figure 5 [28, 32].

\section{Gentest}

\begin{tabular}{|c|c|c|}
\hline Inputs & Assessments & $\begin{array}{l}\text { Optimum Lifestyle and } \\
\text { Medical Plan }\end{array}$ \\
\hline $\begin{array}{l}\text { - Personal information } \\
\text { - Health information } \\
\text { - Living and working conditions } \\
\text { - Lifestyle information } \\
\text {-Nutrition } \\
\text {-Nutritional habits } \\
\text { - Food consumption data using } \\
\text { Gentest Food Portion Atlas } \\
\text { - Physical activity and exercise } \\
\text { - Smoking } \\
\text {-Anthropometric and bioimpedance } \\
\text { measurements } \\
\text { - Biomarkers } \\
\text { - Genotype information }\end{array}$ & $\begin{array}{l}\text { - Nutritional assessment } \\
\text { - Body composition assessment } \\
\text {-Assessment of physical activity } \\
\text { and exercise } \\
\text {-Assessment of causes of smoking } \\
\text { - Assessment of current health } \\
\text { information } \\
\text { - Genotype assessment } \\
\text { - Assessment of biomarkers } \\
\text { - Assessment of risks of common } \\
\text { chronic diseases, i.e. heart attack, } \\
\text { stroke, type } 2 \text { diabetes, } \\
\text { osteoporosis, cancers (lung, breast, } \\
\text { prostate, colon and stomach) }\end{array}$ & $\begin{array}{l}\text {-Medical evaluation } \\
\text {-Medical follow up plan } \\
\text { - Programme for optimum } \\
\text { body composition } \\
\text {-Nutritional } \\
\text { recommendations } \\
\text { - Menu plans } \\
\text { - Exchange options } \\
\text { - Supplements } \\
\text {-Physical activity and } \\
\text { exercise recommendations } \\
\text { - Exercise programmes } \\
\text { - Recommendations on } \\
\text { quitting smoking }\end{array}$ \\
\hline \multicolumn{3}{|c|}{ Follow up plan (optional) } \\
\hline $\begin{array}{l}\text { Neekly, monthly, yearly evalua } \\
\text { Revisions of the plans }\end{array}$ & & \\
\hline
\end{tabular}

\section{Figure 5: Conceptual framework of Gentest}

Source: Cesuroglu, et al.,2009 [28]

The purpose of Gentest model is prevention of the major chronic diseases and their complications, through all stages of their development and progression, i.e. from the point where there is no disease to the point where there is fully established disease and further towards its complications. Having a look at the disease development curve (Figure 4), the practice model aims to catch the individuals during the stages on the left side, i.e. as early as possible, in order to slow down, delay, or if possible, reverse the progression towards the disease threshold. For this purpose, the model attempts to include as much as possible of all determinants of health to make a 360 degree assessment of health of the individual.

Gentest was developed by GENAR Institute for Public Health and Genomics Research, which was established in 2004, in Hacettepe University Science Park, Ankara Turkey. GENAR aimed to transform scientific developments in the area of biotechnology, especial- 
ly genetics and genomics, into products and services that improves human health, quality of life and performance, and extends life span. At the time of its establishment, it was the third public health genomics center in Europe. The author of this dissertation gained firsthand experience with the model while serving as the Research and Development Coordinator of GENAR in 2005-2010.

Gentest was identified as a best practice model for public health genomics in Europe by the Public Health Genomics European Network (PHGEN) in 2008. It was also successfully piloted by authorized practitioners (mainly physicians and some dieticians) as a service provided to more than 500 individuals in Turkey (as of 2010). These practitioners complied with a specific set of criteria and were authorized for application of this model after successful completion of a training course on personalized medicine and nutrition by the Turkish Society of Public Health Genomics and Nutrigenetics/ Personalized Medicine (TOGEN) in collaboration with the GENAR Institute.

Further information on Gentest and how it is implemented can be found in Chapter 6 and Appendix of this dissertation.

Based on the current trends and developments in the world, feedback of scientific and industrial communities on the model, and past experiences with the practice model Gentest, it was identified that the main implementation area for such a preventive health care product would be primary care services in Europe. Within this setting, it can be provided by general practitioners/ family physicians. However, there has been no example of such a preventive personalized health care model that has been provided to large populations. Thus, there is a need for policy research in order to see how this model can be implemented in health systems, in particular primary care settings, in Europe.

\section{The aim and structure of the dissertation}

This $\mathrm{PhD}$ research has been driven by the societal challenge of the burden of chronic diseases, as well as the opportunities brought by personalized medicine and health care to tackle them. The example used in this dissertation, Gentest, was developed as a personalized health care practice for prevention of chronic diseases. Now the question is how it can be integrated into in primary care services in Europe. Thus, the aim of this dissertation is to explore how a personalized health care model, such as Gentest, can be integrated in primary care services in European health systems, for prevention of chronic diseases.

Under this broad question, 'integration' can be split into three elements: where to integrate, what to integrate, and how to integrate? The dissertation is organized in three parts, each addressing one of these elements, as introduced below. 
Where to integrate? - In order to effectively identify issues related to integration, we first need to understand the setting that the practice model will be integrated into. This setting, in the broadest sense, is health systems. Therefore, Part I defines and introduces a framework for health systems, in its larger context of public health. This will provide the underlying basis needed when identifying integration issues.

What to integrate? - Integration depends on the nature and properties of the intervention. The future vision of personalization of medicine and health care is not addressed with standard terms, nor defined in a standard way. Moreover, there is no clear description of what kinds of practices constitute personalized medicine and health care. Therefore, to have a good understanding of what will be integrated, Part I first looks at the broader context of personalized medicine and health care and, then, introduces the practice model that we aim to integrate into health systems.

How to integrate? - Once we achieve a deeper understanding of 'where to integrate' and 'what to integrate', we need to identify how to approach the integration problem. What are the issues we need to look at when investigating how such a practice model can be integrated into health systems? How can we identify them? Upon investigating existing approaches to answer these questions, Part III proposes a solution: a systematic approach that is built on the health system framework introduced in Part I; and takes the practice model Gentest introduced in Part II as its case example.

After this introduction (Chapter 1), the dissertation presents 6 articles (Chapters 2-7), organized in three parts as laid above, and followed by a general discussion (Chapter 8). The appendix provides additional information on the practice model that is used as an example in this dissertation. The overall structure is presented in Figure 6 and each part is described below.

\section{Part I - Where to integrate?}

In scientific and grey literature, discussions on integration of personalized practices have mostly been focusing on the various separate parts of the health system in a piecemeal way. However, for their effective integration, it is important to see the big picture first. Therefore, in this chapter we took a step back and saw that the whole debate on 'where to integrate' needs to build on the concepts of 'public health' and 'health systems'. However, there is confusion around these concepts and how they are interrelated. If we don't have clarity about them, we cannot identify the issues related to integration of a personalized practice into a health system effectively.

As a solution, Chapter 2 took the endeavor to achieve a coherent terminology for policy makers in local, national and international levels. It does so by integrating the concepts of public health, including health system and its components, into a single coherent framework called Health Globe. Among others, components of health system were described in 
details. As it puts forward a comprehensive approach, this framework can be used to identify all issues relevant to integration in a health system. Thus, Health Globe provides the required framework for identification and assessment of issues related to integration in Part III, which will explore how the practice model can be integrated into health systems.

Among various health systems around the globe, the one of the United States of America (USA) is positioned as the most progressive one in terms of medical technologies. USA also seems to be a leader in development of the concepts and practices of personalized medicine and health care, as most of the existing examples and publications originate there. In order to understand the health system context of these 'personalized' practices, we investigated the status of U.S. health 'system' in Chapter 3. In the context of this dissertation, Chapter 3 serves to the purpose of taking lessons from USA when discussing how to and how not to integrate personalized practices in Europe. In the course of the editorial process of the publication, the paper was abridged and the sections where we explained the shortcomings of the U.S. system in relation to broader policy values were taken out. To maintain coherence within this dissertation, these sections, which were omitted in the original publication, were put back into two boxes in Chapter 3 with an explanatory note.

\section{Integration of a Personalized Health Care Model into Health Systems and Policies in Europe}

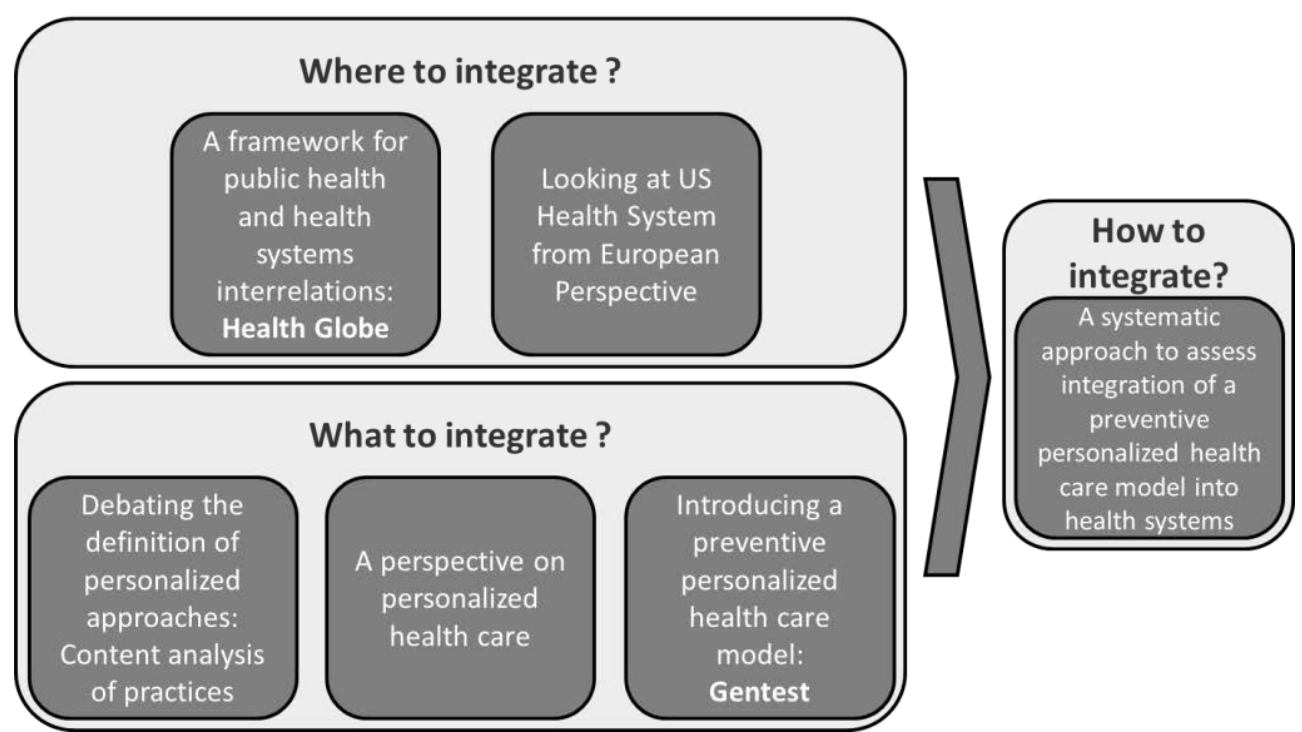

Figure 6: Structure of the dissertation parts and chapters 


\section{Part II - What to integrate?}

The concepts of personalized medicine and health care are new and do not have standard and coherent definitions that are accepted across disciplines, sectors and settings. Without knowing the broad picture of the field, it wouldn't be possible to place Gentest, the practice model that is used as an example in this dissertation, in the right context. Therefore, the broader context of personalized approaches is addressed in Part II, as well as the practice model.

In our efforts to identify the broader context, we realized that there are a variety of terms and definitions for personalized approaches, used in ambiguous and inconsistent ways. They mostly have been made in a top-down manner by influential people or committees. Since the starting point of the PhD research is a practice model that was developed and piloted in real life, in Chapter 4, we chose to provide a different angle on the debate with a 'practice-based perspective', or in other terms a 'bottom-up perspective' by analyzing the content of 'personalized' practices published in the literature. These practices were identified with a systematic search, screening and eligibility strategy. After the content analysis, we reported the notions they imply for the definition of the personalized approaches and saw that 'personalized' practices are not a homogeneous group. Additionally, using three country examples (USA, France and Taiwan) issues related to integration of practices into health services were discussed. While doing that, we used the earlier work on U.S. health system (Chapter 3). Eventually, Chapter 4 highlighted that health systems and policies of countries play a crucial role for successful integration.

Chapter 4 showed the various facets of the personalized approaches but it was Chapter 5 where a working definition of personalized health care was proposed. The article clarified what commonly used terms in the field, including personalized medicine, personalized health care and individualized medicine, meant to the authors. It also conveyed some of the underlying principles that were used in Gentest, including the need to cover all relevant determinants of health, not just genes, when making health care personalized.

Since 'personalized' practices are not a homogeneous group that can be covered in a generic way (as seen in Chapter 4), when developing a systematic approach to identifying issues related to integration of these practices, we needed to focus on a specific example. Chapter 6 provided us this example and introduced Gentest as a practice model for personalized health care. The article summarized the underlying societal need that led to development of the practice model, the approach of GENAR, the content of the practice model and how it was introduced and piloted in Turkey.

\section{Part III - How to integrate?}

After Part I that describes the setting, i.e. health systems, and Part II that focuses on the personalized preventive practice model in its wider context of personalized health care, 
Part III explores how the practice model can be integrated into the setting, i.e. health system.

If we want to integrate Gentest into primary care services in Europe, what are the issues that we should investigate? How can we approach this in a systematic and analytical way? What areas of the health system of the country should be investigated? Chapter 7 aimed to answer these by developing a systematic approach that was built on the health systems framework introduced as a part of Health Globe in Chapter 2. As we've seen in Chapter 4, 'personalized' practices are diverse and cannot be covered with a generic approach. Therefore, Gentest, introduced in Chapter 6, was used to exercise and identify which issues should be covered. Chapter 7 was enriched with examples provided from the Netherlands and the UK, as well as other European settings as necessary. All in all, this chapter provided a systematic approach to identification and assessment of the issues related to integration of the practice model into primary care services in Europe. This method can be applied to other practices and countries and, thus, provide a solution to the broader problem of the integration of personalized medicine and health care practices into health systems.

\section{General Discussion}

Chapter 8 takes a step back and explains why innovations in personalized medicine and health care must be coupled by innovations in the health system and vice-versa to effectively prevent chronic diseases. Room for innovation is addressed for both fields, i.e. health system, as well as its broader context of public health, and personalized medicine and health care.

The practice model Gentest is used as a vehicle in this dissertation in order to base the debate around integration issues on a concrete real life case. In the General Discussion, reflections are made on this choice and how the methodology of using a real life case may contribute to research in this field.

\section{Appendix}

The practice model is used as a practical case, a 'vehicle' in this dissertation, rather than being the central focus of the dissertation in terms of its technical and operational content. For readers who would like to get a better overview of the practice model with its strengths and weaknesses, some additional information is provided in the Appendix to complement Chapter 6, where Gentest was introduced. 


\section{References}

1. Institute for Health Metrics and Evaluation. The Global Burden of Disease: Generating Evidence, Guiding Policy. Seattle, WA: IHM E; 2013. 50 p.

2. Global Health Estimates 2014. Summary Tables, Deaths By Cause, Age and Sex, 2000-2012. Geneva, Switzerland: World Health Organization; 2014. Available from: http://www.who.int/healthinfo/global_burden_disease/en/.

3. Global status report on noncommunicable diseases 2014. Geneva, Switzerland: World Health Organization; 2014. 298 p.

4. Global status report on noncommunicable diseases 2010. Geneva, Switzerland: World Health Organization; 2011. $176 p$.

5. Institute for Health Metrics and Evaluation. The Global Burden of Disease: Generating Evidence, Guiding Policy - European Union and Free Trade Association Regional Edition. Seattle, WA: IHME; 2013. 67 p.

6. Deaths and Disability Adjusted Life Years (DALYs) in EU and EFTA countries for 2010 in Global Burden of Disease Database [Internet]. IHME. 2013 [cited 7 April 2015]. Available from: http://vizhub.healthdata.org/gbd-compare/.

7. The 2012 Ageing Report: Economic and budgetary projections for the $27 \mathrm{EU}$ Member States (2010-2060). Brussels: European Commisssion; 2012. 472 p.

8. Ageing population: projections 2010 - 2060 for the EU27: European Parliament; 2013. Available from: http:// www.europarl.europa.eu/eplibrary/LSS-Ageing-population.pdf.

9. Busse R, Blümel M, Scheller-Kreinsen D, Zentner A. Deaths and burden of chronic disease in Europe. Tackling chronic disease in Europe: strategies, interventions and challenges. Copenhagen: World Health Organization 2010, on behalf of the European Observatory on Health Systems and Policies; 2010.

10. Bloom DE, Cafiero ET, Jané-Llopis E, Abrahams-Gessel S, Bloom LR, Fathima S, et al. The Global Economic Burden of Noncommunicable Diseases. Geneva: World Economic Forum; 2011.

11. Busse R, Blümel $M$, Scheller-Kreinsen D, Zentner A. Economic consequences of chronic disease. Tackling chronic disease in Europe: strategies, interventions and challenges. Copenhagen: World Health Organization 2010, on behalf of the European Observatory on Health Systems and Policies; 2010.

12. The 2014 EU Summit on Chronic Diseases. Brussels, 3 and 4 April 2014 - Conference Conclusions. Luxembourg: European Commission; 2014. Available from: http://ec.europa.eu/health/major_chronic_diseases/docs/ev_20140403_mi_en.pdf.

13. Political Declaration of the High-level M eeting of the General Assembly on the Prevention and Control of Non-communicable Diseases: United Nations General Assembly; 2012. Available from: http://www.who.int/nmh/events/un_ncd_summit2011/political_declaration_en.pdf.

14. Global Action Plan for the Prevention and Control of NCDs 2013-2020. Geneva2013.

15. Gaining health. The European Strategy for the Prevention and Control of Noncommunicable Diseases. Copenhagen: World Health Organization Regional Office for Europe; 2006.

16. Plochg T, Klazinga NS, Schoenstein M, Starfield B. Reconfiguring health professions in times of multimorbidity: Eight recommendations for change. 2011. In: Health Reform: Meeting the Challenge of Ageing and Multiple Morbidities [Internet]. OECD Publishing. Available from: http://dx.doi.org/10.1787/9789264122314-7-en. 
17. Smith $\mathrm{R}$, Corrigan $\mathrm{P}$, Exeter $\mathrm{C}$. Countering non-communicable disease through innovation: Report of the Non-Communicable Disease Working Group 2012: Imperial College London; 2012.

18. Corrigan P, Exeter C, Smith R. Innovate or die. Bmj. 2013 2013-03-27 11:26:30;346.

19. Department of Health UK. Innovation, Health and Wealth, Accelerating Adoption and Diffusion in the NHS. UK: Department of Health; 2011. 34 p.

20. The Commission's Communication: "Taking forward the Strategic Implementation Plan of the European Innovation Partnership on Active and Healthy Ageing": European Commission; 2012. Available from: http://eur-lex.europa.eu/legal-content/EN/ALL/?uri=CELEX:52012DC0083.

21. Lalonde M. A new perspective on the health of Canadians - a working document. Ottawa: M inister of Supply and Services Canada; 1974.

22. Engel $G$. The need for a new medical model: a challenge for biomedicine. Science. 1977 April 8, 1977;196(4286):129-36.

23. Evans RG, Stoddart GL. Producing health, consuming health care. Soc Sci Med. 1990 $/ / ; 31(12): 1347-63$.

24. Committee on Capitalizing on Social Science and Behavioral Research to Improve the Public's Health - Division of Health Promotion and Disease Prevention. Promoting Health: Intervention Strategies from Social and Behavioral Research. Smedley BD, Syme SL, editors: National Academy Press; 2000.

25. The Future of the Public's Health in the 21st Century: The National Academies Press; 2003.

26. Dahlgren G, Whitehead M. "Dahlgren G, Whitehead M (1993). Tackling inequalities in health: what can we learn from what has been tried? Working paper prepared for the King's Fund International Seminar on Tackling Inequalities in Health, September 1993, Ditchley Park, Oxfordshire. London, King's Fund." In: European strategies for tackling social inequities in health: Levelling up Part 2. Copenhagen: World Health Organization Regional Office for Europe 2006.

27. Sameroff A. A Unified Theory of Development: A Dialectic Integration of Nature and Nurture. Child Development. 2010;81(1):6-22.

28. Cesuroglu T, Karaca S, Erge S. A practice model for personalized healthcare with a public health genomics perspective. Personalized M edicine. 2009 2009/09/01;6(5):567-77.

29. Snyderman R, Yoediono Z. Prospective care: a personalized, preventative approach to medicine. Pharmacogenomics. 2006 2006/01/01;7(1):5-9.

30. Ford ES, Ajani UA, Croft JB, Critchley JA, Labarthe DR, Kottke TE, et al. Explaining the Decrease in U.S. Deaths from Coronary Disease, 1980-2000. New Engl J M ed. 2007;356(23):2388-98.

31. Cesuroglu T, van Ommen B, Malats N, Sudbrak R, Lehrach $H$, Brand A. Public health perspective: from personalized medicine to personal health. Personalized Medicine. 2012 2012/03/01;9(2):115-9.

32. Savas B. A Case in Commercial Applications of Nutrigenetics. Abstract A31. In: 1st Congress of the International Society of Nutrigenetics/Nutrigenomics (ISNN). J Nutrigenet Nutrigenomics. 2007;1(1-2):72. 
PART I

WHERE TO INTEGRATE? 
Essentially, all models are wrong, but some are useful.

George E.P. Box \& Norman R. Draper 


\section{Chapter 2}

\section{A framework for public health and health systems interrelations: Health Globe}

B. Serdar Savas and Tomris Cesuroglu

Health Globe: A Proposal for a Coherent Terminology of Public Health Concepts. Submitted. 


\begin{abstract}
There is confusion around concepts of public health, including health system and its components, and how they are related to each other. In this paper we build on the existing literature and aim to integrate these concepts, which have been defined in separate contexts, into a single framework called 'Health Globe' and define them coherently.

In Health Globe, the broad approach to health brought by World Health Organization's 'Health for All' vision is defined as public health and it is illustrated with a globe. Thus, all activities that directly or indirectly affect individuals' health, including all medical and nonmedical interventions, governmental decisions and practices, and activities of the public sector, the private sector and NGOs, should fall within the scope of 'public health'. Activities under the mandate of the ministry of health have been defined as 'health system', which is a part of the Health Globe.
\end{abstract}

Within the Health Globe, there are other constituents that determine health, such as prerequisites for health and non-health systems' health related activities. Governance for health is what keeps all the elements and activities together aligned towards the common goal: "the attainment by all peoples of the highest possible level of health". 


\section{Introduction}

Environmental, political, economic, social and technological changes all have important implications for human health. In this context, in order to enable the countries to tackle current and newly-arising health challenges, guidance is required crucially from international/ intergovernmental bodies on health matters, including World Health Organization (WHO) as the leading one. However, a consensus is lacking on the meaning of concepts of public health, including health system and its components, and the ways public health and health systems relate to each other. It is not possible to provide proper guidance globally if the interrelations of these basic concepts that are used so frequently in the international fora are not clear to all involved parties.

The scientific and grey literature is rich in definitions and descriptions of concepts that are related to public health, health system and its components, and some major areas such as governance for health. Certain groups might consider that there is a fair consensus on one or more of these for different contexts or purposes. However, when it comes to bringing them together to use in practice, various areas of confusion within the terminology appear. There is no conceptual framework that brings all these together in a coherent way and explains how they are interrelated.

The overall aim of this paper is to contribute to the goal of achieving a coherent terminology, mainly from the perspective international/ intergovernmental bodies on health matters, in particular the WHO. For that, we take the endeavor to integrate the concepts of public health, including health system and its components, into a single conceptual framework, explaining how they are interrelated, and provide a visual depiction. We will first make a brief review of the key concepts we will use and highlight the problems with this terminology and causes of confusion. We will then propose our conceptual framework and some further clarifications as possible solutions. Then, we will discuss and conclude with the possible added values of Health Globe in the field.

\section{Main concepts and confusion within the terminology}

In this section, we provide the conceptual basis of the components that will be used in the framework we'll introduce in the following section.

\subsection{Overview of the main terms and definitions}

A brief historical context and the list of concepts that will be used are provided below. We summarize some of the prominent and/or conflicting definitions that we've identified for each.

\section{Context and purpose of 'Public Health'}

The Global Strategy for Health for All by the Year 2000 [1], encapsulates the paradigm shift that took WHO from a 'combating disease' approach to one that considered health 
policy in the broadest sense. WHO's 'Health for All' (HFA) defined health not as the sole domain of national ministries of health, but as a phenomenon that is shaped by the entirety of a nation's activities. As a result, numerous different areas came within the scope of HFA strategies: peace as a prerequisite for health, agricultural policy, income distribution, housing policies, national education, urbanization, etc.

The implementation of HFA strategies necessitates that all societal decisions' implications on health should be considered. Legislation and regulation, governmental implementations of policies, juridical decisions, and the actions of the public and private sector and non-governmental organizations (NGOs), all need to comply with the objective of "the attainment by all peoples of the highest possible level of health" [2]. This approach to decision making refers to 'governance for health' (see later).

Health is not only the product of activities with the primary intention for health. Ottawa Charter introduced that there are pre-requisites for health, which consist of peace, shelter, education, food, income, a stable eco-system, sustainable resources, social justice, and equity [3]. Non-health systems' health related activities are those that don't have health as their primary mandate, but have the potential to influence health, such as traffic control, the taxation of tobacco products, the regulation of agricultural products, etc.

The importance of non-health sectors' activities on the health status of the population is emphasized once more with the introduction of Health in All Policy (HiAP) by the Finnish EU Presidency in 2006 [4]. It emphasizes the fact that all policies such as transport, housing, the environment, education, fiscal policies, tax policies and economic policies have an impact on health. Health is determined by not only health related activities, but also the social and economic context of individuals, emphasizing the social determinants of health as an epidemiological concept [5].

\section{Public Health}

One of the earliest and most prominent public health definitions is made by Winslow. This is the largest possible definition within the context of his time (1920): "the science and art of preventing disease, prolonging life, and promoting physical health and efficiency through organized community efforts for the sanitation of the environment, the control of community infections, the education of the individual in principles of personal hygiene, the organization of medical and nursing services for the early diagnosis and preventive treatment of disease, and the development of the social machinery which will ensure to every individual in the community a standard of living adequate for the maintenance of health".

Until the 1970s, WHO's activities focused primarily on combating disease. Beginning in that decade, HFA policies were adopted in order to attain WHO's constitutional objective of "the attainment by all peoples of the highest possible level of health" [2]. This approach 
brought health's political, economic and social dimensions to the fore, with a particular emphasis on equity. This visionary paradigm shift led to the broadening of the scope of public health. In 1988, Acheson defined public health as "the science and art of preventing disease, prolonging life and promoting health through the organised efforts of society". This definition captures also the HFA approach, and remains complete to this day. That said, attempts have been made to elaborate this definition further and emphasize certain key terms $[6,7]$. It is also of note that, public health encompasses the 'health services', including more narrow concept of 'medical care' [8, 9], as a part of the 'organised efforts of society' in Acheson's definition.

Terms similar to 'public health' are used in different contexts with different meanings, and can lead to confusion: The 'public's health' or the 'health of the public' [10] refers to society's health level. 'Public health services' (emphasis on 'public') refers to health services that are offered by the governmental sector or other public bodies [8], as opposed to the private sector. This is analogous to the use of 'private hospitals' versus 'public hospitals', or 'private health insurance' versus 'public health insurance'. 'Public health services' (emphasis on 'public health') refers to special types of health services. For example, the sanitary-epidemiologic services offered under the former Soviet Union's Semashko scheme are an example of public health services.

Within the context of the Region of Americas (Pan American Health Organization - PAHO), and especially in the USA, the debate has focused on the assignment of a mandate for public health. While early discussions emphasized the responsibilities of public authorities $[11,12]$, the current understanding has settled on a scope that defines society as a whole as actors (i.e., including the private sector) and, therefore, bearing certain responsibilities in the area of public health $[13,14]$. As a part of this discussion, the 10 essential public health services was defined by the Public Health Functions Steering Committee in US [15]. These outline the minimum responsibilities that must be borne by public authorities.

The scope of public health is often narrowed as a part of public health education. This is primarily because such education is provided by medical schools / faculties, and is mislabeled as 'public health', when the content is actually 'community/social medicine' [9]. Similarly, in instances where public health education is provided outside of medical faculties, health services have been moved out of public health's scope, and the term has been limited to the study of environmental and social influences on health and disease, focusing on disease prevention and health promotion [16].

The new European health policy framework developed by the World Health Organization Regional Office for Europe (WHO Europe), Health 2020, initially introduces public health as "...a dynamic network of stakeholders at all levels of society and aims to support action with unity of purpose across the Region." in its Foreword by the Regional Director, $\mathrm{Z}$. Jakab [17]. This approach takes public health at a higher level encompassing all relevant 
actors involved in health. Nevertheless, the rest of the document, as well as other related initiatives of WHO Europe [18], uses 'public health' in the context of a (core) set of services/ operations that are provided in population level, which are apart from health care services.

\section{Health System and its Components}

Various definitions and frameworks on health system have been developed, in particular to provide a basis for assessment of its performance within various countries. A highly coherent and influential one was developed by Murray and Frenk in 2000 [19]. They drew the borders of the health system with 'health actions', which is any set of activities whose primary intent is to improve or maintain health. "And a health system includes the resources, actors and institutions related to the financing, regulation and provision of health actions." [19] This concept was reflected to the framework of WHO on health systems performance [20] and was further elaborated in 2007 to mean "... all organizations, people and actions whose primary intent is to promote, restore or maintain health" [21].

In 2002, PAHO had stated that "Public health is an integral part of the health system which is understood to be the interventions carried out in society with health as the primary goal" [13]. This definition is problematic in terms of both its scope and purpose. By putting it under the health system, it reduces 'public health' into a set of services/ functions/ tasks, and narrows down its meaning.

In 2008, the Tallinn Charter defined a health system as follows: "Within the political and institutional framework of each country, a health system is the ensemble of all public and private organizations, institutions and resources mandated to improve, maintain or restore health. Health systems encompass both personal and population services, as well as activities to influence policies and actions of other sectors to address social, environmental and economic determinants of health." [22] Thus, by going beyond 'the activities whose primary purpose is health', Tallinn definition crossed the demarcation line made in 2000 and entered the non-health sectors' territories when defining health system.

In their conceptual framework, Murray and Frenk defined goals and functions of health systems. Three main goals are health, responsiveness and fairness in financial contribution. Four basic functions that need to be performed by every health system are financing, provision, stewardship and resource generation. This framework provided the basis of WHO's health system performance assessment framework, which was used in World Health Report 2000 [20].

In the context of WHO's guidance development for health systems strengthening, in 2007, WHO simplified this into six building blocks, which are prioritized areas of health systems that commonly needs strengthening: Health services; health workforce; health infor- 
mation system; medical products, vaccines and technologies; health financing; and leadership and governance [21].

A more recent work by Atun and Menabde emphasized that health systems are complex dynamic systems and effective interventions can be developed only when they are analyzed together with their demographic, economic, political, legal and regulatory, epidemiological, social and technological contexts which they interact with [23].

There are also various other frameworks or tool sets that are, for example, developed to measure, assess or compare health systems' performance [24], to guide health reforms [25], to analyze the investments for health systems strengthening [26], or for the context of specific countries [27].

\section{Governance for Health}

The use of the concept of (good) governance [28] became widespread at the end of the 1980s, making its way into WHO terminology in the 1990s, where it was used in the context of public health $[20,29,30]$. Despite the growing interest in this concept globally, a consensus on a clear and actionable description of this function has been lacking [31]. In a recent report commissioned by WHO Europe in the context of Health 2020, Kickbusch and Gleicher define governance for health as "the attempts of governments or other actors to steer communities, countries or groups of countries in the pursuit of health as integral to wellbeing through both a 'whole-of-government' and a 'whole-of-society' approach" [32]. In the broader context of HiAP, governance can be considered as the verb for acting on social determinants of health to achieve HiAP [5]. The whole-of-government and wholeof-society approaches have been embraced in various recent policy documents and reports $[17,33,34]$.

Governance is a term that is distinct from 'government' [35]. It should further be noted that 'government' does not refer solely to the executive branch, but also encapsulates the state's legislative and judiciary functions.

\section{Stewardship (health system function)}

Stewardship defines strategic directions for the health system. It also sets, implements, and monitors the rules of the health system [19]. Stewardship assures a level playing field for all actors in the system [19]. Stewardship can be divided into six subfunctions: overall system design, performance assessment, priority setting, inter-sectoral advocacy, regulation, and consumer protection [19, 36].

\section{Health Services (health system function)}

Provision of health services has been described as "combination of inputs into a production process that takes place in a particular organizational setting and that leads to the delivery of a series of interventions" [19]. Murray and Frenk clarify the conventional dif- 
ference between personal and non-personal health services: "The former refer to services that are consumed directly by an individual, whether they are preventive, diagnostic, therapeutic or rehabilitative, and whether they generate externalities or not. The latter refer to actions that are applied either to collectivities (e.g., mass health education) or to the non-human components of the environment (e.g., basic sanitation)."

\section{Health System Financing (health system function)}

"Health system financing is the process by which revenues are collected from primary and secondary sources, accumulated in fund pools and allocated to provider activities" [19].

\section{Resource Generation (health system function)}

Resources in a health system cover human resources, physical resources such as facilities and equipment, and knowledge [19].

\section{Health Gain}

Health gain is an increase in the measured health of an individual or population, including length and quality of life [37].

\section{Equity}

"Equity in health implies that ideally everyone should have a fair opportunity to attain their full health potential and, more pragmatically, that no one should be disadvantaged from achieving this potential, if it can be avoided. The term inequity ... refers to differences in health which are not only unnecessary and avoidable but, in addition, are considered unfair and unjust." [38]

\section{Environmental Health Services}

In the HFA context, environmental health was defined in the broadest sense as "... those aspects of human health, including quality of life, that are determined by physical, chemical, biological, social and psychosocial factors in the environment." and the environmental health services as implementation of the environmental health policies, without restriction to any sector [39].

\section{Primary Care Services}

Primary care services are the first level of contact that individuals and the family have with health care services, bringing health care as close as possible to where people live and work. These form the first element of a continuing health care process [40, 41]. It should be noted, however, that the scope of the 'primary care services' is narrower than the scope adopted for 'primary health care' in the Alma-Ata declaration [41, 42].

\subsection{Main confusion areas within the terminology}

The terminology around the concepts of public health, including health system and its components, is confusing in various points, such as listed below: 
- Public health concept is used inconsistently, even within WHO, in a way that it means different things in different contexts and time frames, in particularly owing to the use and meaning of 'public' in the noun phrase.

- The relationship of public health with health systems is not clear. This leads to confusions in communication in the field. Some consider that 'public health' or 'health system' contains the other and some consider them equally separate (public health services vs. health (care) services).

- Clarity on the levels of health services is lacking, in particular in relation to what is considered as 'public health services/functions/operations'.

- The place and content of primary care services within the health services is not always clear.

- Governance and stewardship concepts are (still) used inter-changeably, leading to confusion on the responsibilities of ministries of health.

- The growing interest in making health a whole-of-government and whole-ofsociety agenda item is further complicating the clarity of the above-mentioned definitions and creating confusion on who is responsible for which services.

Overall, various concepts are defined for specific purposes, which might be coherent and well valid for their own context. However, they don't make a whole picture when they are brought together in a piecemeal way. There is a need for coherent overarching conceptual framework that integrates them and clarifies their meanings.

\section{Proposed solution}

\subsection{Health Globe}

In this section, we build on the definitions made in the previous section and propose 'Health Globe' as a coherent framework that integrates different elements of public health, including health system and its components, into a single framework. The HFA paradigm of WHO is used as an overarching approach to health, which integrates all components in the Health Globe in Figure 1. It defines the approach adopted by HFA as 'public health' in its entirety. Since Acheson definition is also in line with HFA, it is compatible with Health Globe's approach. Everything that relates to a society's health is thus categorized as a part of public health, in the sense that it aims to attain the highest possible level of health by all peoples.

The involvement of whole-of-society and whole-of-government in health is labeled as governance for health on the Health Globe (the fluid filling the globe in Figure 1), adopting the term in the broadest sense as defined by Kickbusch and Gleicher [32]. Pre-requisites for health and non-health systems' health related activities are depicted with two types of particles as seen in the Health Globe (Figure 1). 


\section{HEALTH GLOBE}
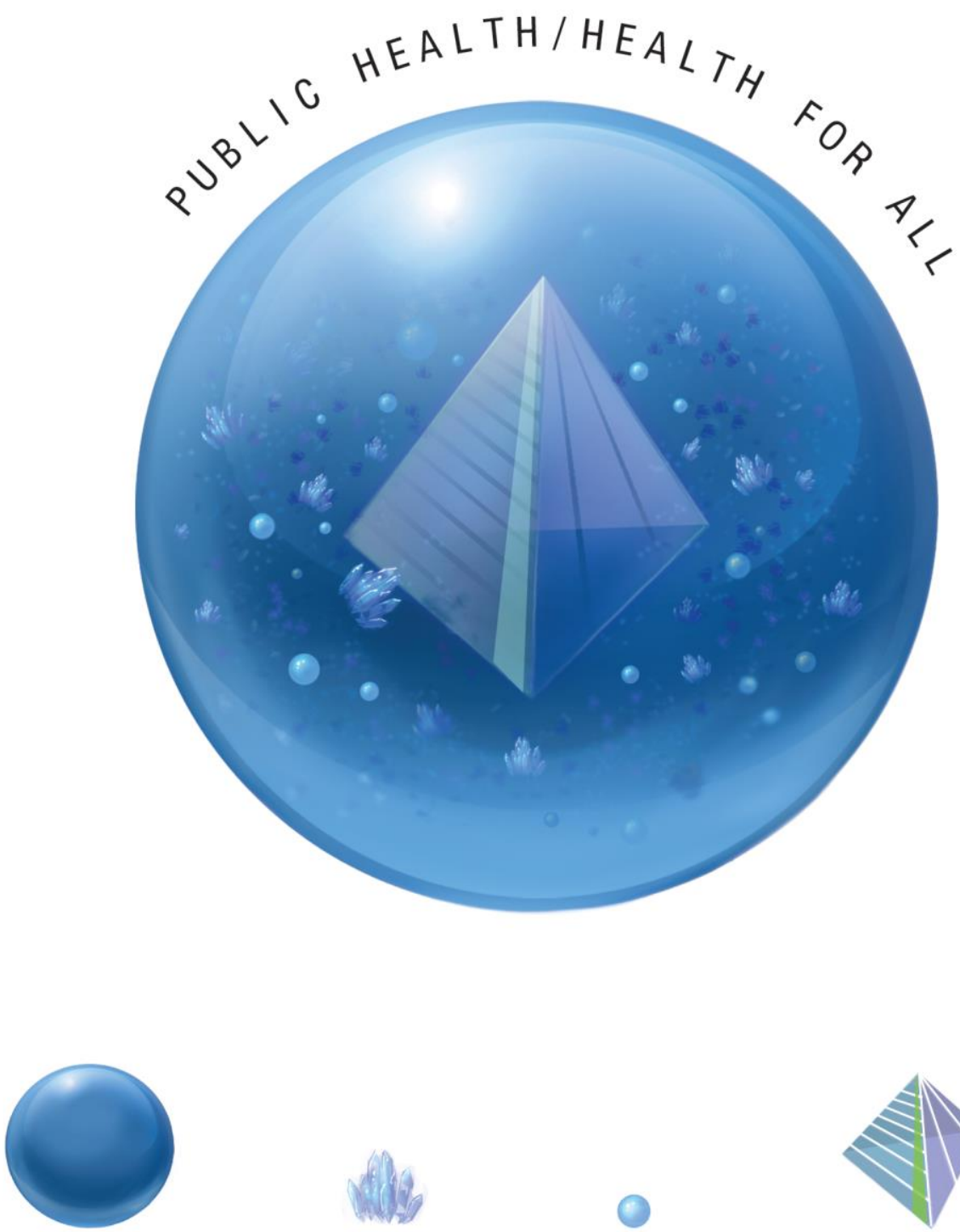

Governance for health

(fluid in the globe)

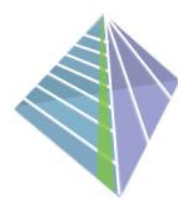

Health system pyramid

\section{Figure 1: Health Globe}




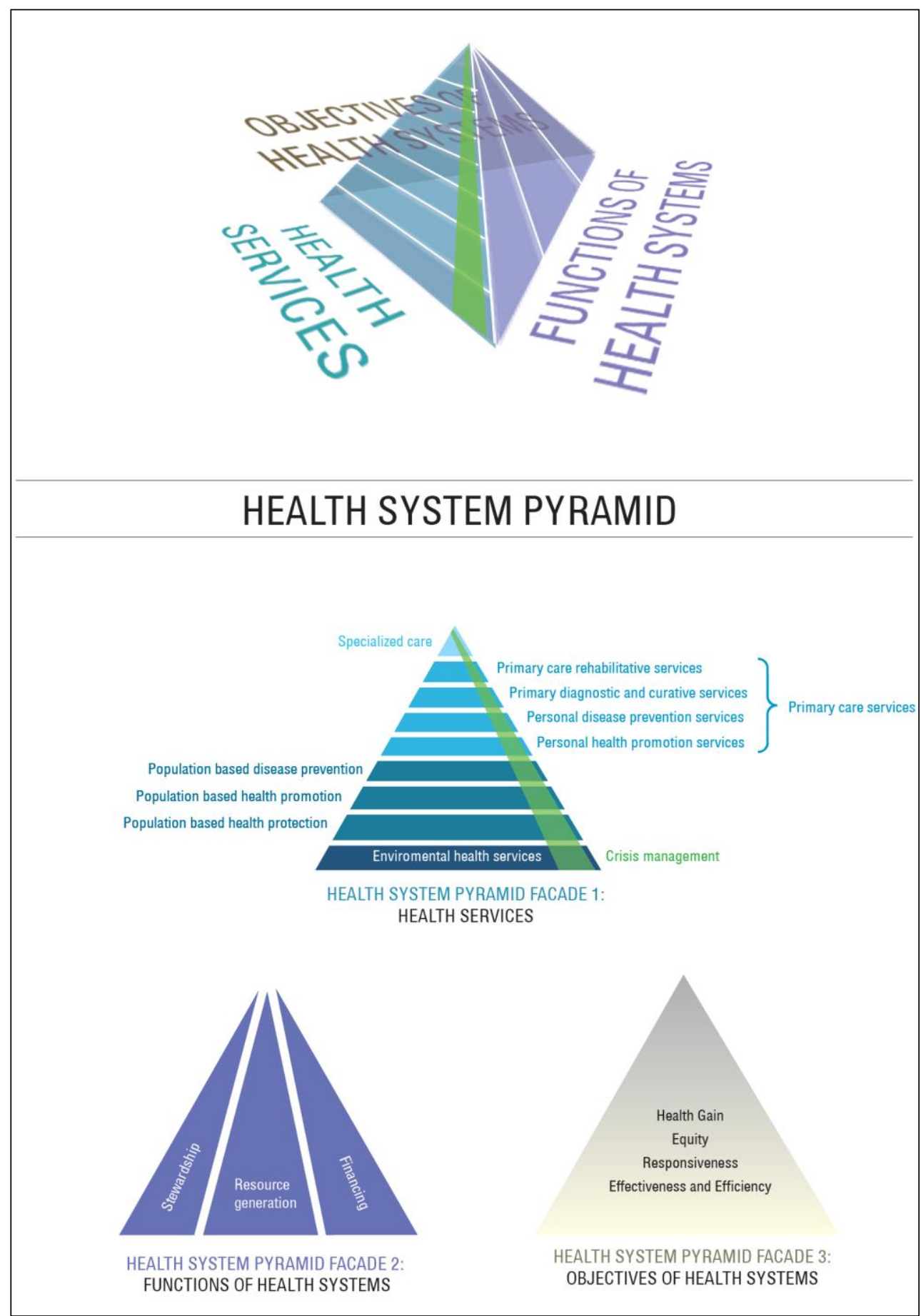

Figure 2: Health System Pyramid and its Three Facades 
The pyramid inside the Health Globe represents the 'health system'. The framework developed by Murray and Frenk provide the coherent basis to structure its components. We adopt this framework to a large extend, while elaborating it at some functions as described below.

As M urray and Frenk described, health system has four key functions: stewardship, financing, service provision and resource generation [19, 20]. In Health Globe, the term 'health services' has been preferred over 'service provision' since it will be covered in more details as lines of services (see later). Here, we adopt the 'health actions' concept [19] and, thus, this pyramid covers activities that fall within the mandates of ministries of health.

For visual clarity, health services function has been depicted in the first facade of the pyramid (Figure 2, Facade 1). The services listed on this surface move up vertically from the bottom: societal to individual; and general to specialized.

As the services go up, they become more specifically a health sector issue and as they go down, they become more multi-sectoral in nature, falling into the domains of non-health sectors as well. For the latter, we cover the tasks of the health system, in particular the contribution of the ministries of health in this pyramid.

Health Globe adopts the clarification made between personal and non-personal health services by Murray and Frenk, and denotes latter as 'population based', in the first facade. Each service type is covered below.

For environmental health services, we adopt a modified version of the definition made by Fitzpatrick and Bonnefoy [39]. They defined the term to cover all activities related to implementation of environmental health policies, by all sectors. For the sake of coherence, in the context of this pyramid, the tasks of the health system, in particular ministries of health, within the environmental health services are covered.

Population based health protection initiatives refer to the regulation of activities that have the potential to damage people's health. These include, among others, the regulation of food additives, safety standards for tools, including toys, and rules such as mandatory seat-belt use in motor vehicles. The health system may not be the one that operates or executes these functions, but play an important role in their realization by creating awareness, generating and disseminating knowledge, developing guidelines and providing advisory and consultancy functions.

Population based health promotion includes initiatives that target the whole society to combat unhealthy behaviors, as well as the promotion of healthy nutrition and physical activity. Depending on the context of the countries, the role of non-health sectors might be more prominent in some of these activities and health system may carry out the tasks 
that support the non-health sectors, as listed above for population based health protection.

The most common examples for population based disease prevention include the vaccination of school children, mass screening campaigns targeting certain types of diseases, disease awareness initiatives and prevention of epidemics.

We adopt the primary care services definition and clarification that was made in the previous section. Additionally, primary care services include home care, which is also given by a primary care services team. Primary care services cover the following four groups:

A) Services provided to the individual for the promotion of a healthy lifestyle, covering aspects such as nutrition, physical activity, smoking cessation, and psychosocial wellbeing, fall under personal health promotion services.

B) Personal disease prevention services refer to interventions that protect against disease at the individual level. They include personal preventive services conducted by a family physician or general practitioner. Influenza shots for seniors, smear tests for women, the prescription of aspirin for those with high cardiometabolic risk, and travel vaccinations (can be provided in different settings) are some examples of such services.

C) Primary diagnostic and curative services are activities that aim to diagnose and treat diseases within the scope of primary care services.

D) Primary care rehabilitative services are composed mostly of follow-ups to specialized care. These include services such as the rehabilitation of patients after cardiac or orthopedic surgeries. These are coordinated through a primary care service provider.

Specialized care covers services provided by specialized health professionals, usually in hospitals, outpatient or inpatient settings. Such activities were formerly classified as secondary or tertiary care services and referred solely to inpatient care. Since this distinction no longer exists, we group all of these activities under specialized care.

Crisis management involves the health services component of emergencies, natural disasters and other health crises. This includes preparedness for and response to all such situations. All levels of health services have their mandates within this vertical responsibility. However, one should keep in mind that crisis management has a wider scope under the governance for health, including risk reduction measures such as flood mitigation works and appropriate land-use planning.

The second facade of the pyramid shows the functions of the health system (Figure 2, Facade 2): stewardship, resource generation and financing. These enable the provision of health services, which was depicted in Facade 1 . They are represented vertically, since 
they involve all levels of the health services covered in the first facade of the pyramid. We adopt the stewardship definition made by M urray and Frenk, summarized in the previous section. However, we modify its subfunctions to include priority setting, legislation and regulation, steering, administration and management. In general, stewardship can be considered as the primary function of a country's ministry of health. In line with Murray and Frenk, resource generation refers to human resources, physical resources and knowledge. Universities, pharmaceutical and medical technology companies and many other public and private organizations and initiatives can fall under this heading. Murray and Frenk's health system financing definition can be shortly sub-grouped as revenue collection, fund pooling and purchasing.

The third facade of the pyramid depicts the objectives of the health system (Figure 2, Facade 3). Modifying Murray and Frenk's slightly, we set the objectives as health gain, equity, responsiveness, and effectiveness and efficiency. We adopt the health gain and equity definitions quoted in the previous section. Health gain is an increase in the measured health of an individual or population, including length and quality of life [37]. Within the context of health services, equity refers to the idea that everyone should have access to health services in accordance with their needs, and should contribute to the financing of these services in accordance with their means. The third objective is enhancing the responsiveness of the health system to the legitimate expectations of the population, as described by Murray and Frenk [19]. Patient satisfaction and satisfaction of the health workforce also fall under this category.

Effectiveness and efficiency are a health system's economic objectives. Effectiveness is about allocating resources to the right interventions to ensure the maximum level of health gain, whereas efficiency is to use resources in the most convenient way to reduce the costs. Effectiveness is doing the right thing; efficiency is doing the thing right.

\subsection{Further clarifications}

A few areas remit further emphases and/or clarifications.

As for the discussion on whether health system is a part of public health or the other way around, as we have proposed in Figure 1 and in line with the scope adopted by HFA, we are in opinion that the health system is a part of public health. As summarized in Section 2 , confusions are mainly caused by the use of noun phrase and the meaning of 'public' in it. In order to avoid such confusions:

- The term 'public health services' used to cover services not targeting individuals, but populations, could be replaced with the term 'non-personal health services' or 'population based services' [8, 19];

- The term 'publicly provided health services' could be used to refer to health services provided by public entities; 
- Reference to the concept of the 'health of the public' could be made using the term the 'public's health'.

The term 'essential public health services/ functions' $[13,15]$ used in the Americas is compatible with the 'health systems', and encompasses various elements from the three facades of the health pyramid. Similar efforts to define 'public health' as tasks, services, operations or functions across countries are highly likely to yield problematic results because these are eventually (sub)functions of health systems and each country have different structures to fulfil them. Furthermore, it creates confusion on the side of policy makers on what public health is. Is it a set of tasks, or is it the broad approach to population health?

To be more explicit; we criticize use of 'public health' in any way that narrows down its meaning to tasks, functions, operations or services. If the purpose is to list a set of specific services that should be provided by (certain) public agencies, it should be done so with the use of the technical name of the services; for example 'sanitation', 'environmental health' or 'training of health workforce'. The tendency to call a set of 'services' as 'public health' seems to be (historically) rooted in the institutions that provide education on these, as conveyed in section 2.1 .

The terms governance and stewardship has often been used inter-changeably depending on the purpose and perspective of the document in question. However, as clarified in the previous section, stewardship refers to a ministry of health's function within a country's health system. The initiatives conducted by a ministry of health in coordination with other ministries and sectors, which aim to influence health through areas outside of the health system, form the ministry's 'governance for health' activities. Therefore, these terms should not be used inter-changeably. As such, the part of the Tallinn Charter on health and stewardship ("activities to influence policies and actions of other sectors to address social, environmental and economic determinants of health" [22]) should be handled at a higher level, under the definition of governance.

\section{Discussion and conclusions}

In this paper, we proposed Health Globe as an endeavor to integrate concepts of public health into a single framework, in order to achieve a coherent terminology for policy makers and those who guide them internationally. In Health Globe, we provided an understanding of the term 'public health' in line with the HFA vision, where all activities that directly or indirectly affect individuals' health, including all medical and non-medical interventions, governmental decisions and practices, and the activities of the public sector, the private sector and NGOs, falls within the scope of 'public health'. 
A point to note here is the distinction between 'individuals' and the 'public'. Any approach that does not include the health services provided to individuals under public health will both prove inadequate in light of the medical advances of the $21^{\text {st }}$ century, and will be problematic from a policy making perspective.

We paid particular attention to design the framework to be applicable to different parts of the globe and different health systems, and conceptually and practically be relevant to real life contexts. It can in particular be useful for WHO, which serves to member states all around the globe.

We believe that Health Globe might provide an added value to the existing literature and practices in three areas:

First of all, Health Globe invites health policy makers to think on crucial points and revisit the HFA strategy in determining a course for public health policy in the $21^{\text {st }}$ century. The discussions that took place after HFA have not been able to make a significant leap over what HFA described in the broad sense. HFA still provides a robust, technically correct, visionary and up-to-date approach. When new approaches beyond HFA are explored in WHO, its member states or academia, this should be kept in mind.

Second point is that Health Globe serves to the efforts to reach a coherent terminology. When we look at the debates in the last decades, we see that a common language hasn't been established in terms of the terminology and concepts. We believe that Health Globe can be instrumental to solve this problem since it aims to integrate the definitions that have been made in different contexts. There will surely be ones who do not agree with this framework. We invite them to propose alternative frameworks that aim to describe the interrelations of these concepts. In such endeavors, it is crucially important to attain coherence and consistency within these frameworks and relevance with the real life in the field. Professional and institutional conservatism must be avoided, as well.

Finally, Health Globe describes the interrelations of these concepts in a simple visual frame. Visual depictions enable concepts and their interrelations to be understood and communicated more easily. This eventually can facilitate the debate on the terminology.

\section{References}

1. Global Strategy for Health for All by the Year 2000. Geneva: World Health Organization 1981.

2. Constitution of the World Health Organization: World Health Organization 2006. Available from: http://www.who.int/governance/eb/who_constitution_en.pdf.

3. Ottawa Charter for Health Promotion, First International Conference on Health Promotion, Ottawa, 21 November 1986: World Health Organization; 1986 November 17-21, 1986. 
4. Ståhl T, Wismar M, Ollila E, Lahtinen E, Leppo K. Health in All Policies: Prospects and potentials. Finnish Ministry of Social Affairs and Health, European Observatory on Health Systems and Policies, 2006.

5. McQueen DV, Wismar M, Lin V, Jones CM. Introduction: Health in All Policies, the social determinants of health and governance. In: McQueen DV, Wismar M, Lin V, Jones CM, Davies $M$, editors. Intersectoral Governance for Health in All Policies. Observatory Studies Series. 26. Copenhagen: World Health Organization on behalf of the European Observatory on Health Systems and Policies; 2012. p. 3-23.

6. World Health Report 1998: Life in the 21st century - A vision for all. Geneva: World Health Organization; 1998.

7. Pencheon D, Guest C, M elzer D, Gray M. Introduction. In: Pencheon D, Guest C, M elzer D, Gray M , editors. Oxford Handbook of Public Health Practice: Oxford University Press; 2006.

8. Frenk J. The new public health. Annu Rev Public Health. 1993;14:469-90. PubMed PM ID: 8323599.

9. Terris M. Editorial: The distinction between public health and community/social/preventive medicine. Journal of public health policy. 1985 Dec;6(4):435-9. PubM ed PMID: 4093498.

10. Verweij M, Dowson A. The Meaning of 'Public' in 'Public Health'. In: Dawson A, Verweij M, editors. Ethics, Prevention, and Public Health: Oxford University Press; 2009.

11. The Future of Public Health. Washington, D.C.: The National Academies Press; 1988.

12. Essential Public Health Functions. 42nd Directing Council \& 52nd Session of the Regional Committe, Washington, D.C., 25-29 September 2000. (CD42/15): Pan American Health Organization, World Health Organization; 2000 [cited 27 August 2012. Available from: http://www.paho.org/english/gov/cd/cd42_15-e.pdf.

13. Public Health in the Americas: Conceptual Renewal, Performance Assessment, and Bases for Action. Washington, D.C.: Pan American Health Organization - Pan American Sanitary Bureau, Regional Office of the World Health Organization; 2002.

14. The Future of the Public's Health in the 21st Century: The National Academies Press; 2003.

15. Public Health Functions Steering Committee. Public health in America 1994 [11 April 2013]. Available from: http://health.gov/phfunctions/public.htm.

16. "L. White. Healing the schism: epidemiology, medicine, and the public's health. New York: Springer-Verlag; 1991." In: Khoury MJ, Gwinn M, Burke W, Bowen S, Zimmern R. Will genomics widen or help heal the schism between medicine and public health? Am J Prev Med. 2007 Oct;33(4):310-7. PubM ed PM ID: 17888858. Epub 2007/09/25.

17. Health 2020. A European policy framework and strategy for the 21st century. Copenhagen: World Health Organization Regional Office for Europe; 2013.

18. European Action Plan for Strengthening Public Health Capacities and Services: World Health Organization Regional Office for Europe; 2012. Available from: http:// www .euro.who.int/en/what-we-do/ event/first-meeting-of-the-european-health-policyforum/documentation/working-papers/strengthening-public-health-capacities-and-services-ineurope-a-framew ork-for-action.

19. Murray C , Frenk J. A framework for assessing the performance of health systems. B World Health Organ. 2000;78(6):717-31. PubM ed PM ID: 10916909. Pubmed Central PM CID: 2560787.

20. World Health Report 2000: Health Systems: Improving Performance. Geneva: World Health Organization; 2000. 206 p. 
21. Everybody's business: Strengthening health systems to improve health outcomes: WHO's framework for action. Geneva: World Health Organization; 2007. 44 p.

22. The Tallinn Charter: Health Systems for Health and Wealth. Adopted at WHO European Ministerial Conference on Health Systems: "Health Systems. Health and Wealth" Tallinn, Estonia, 27 June 2008.: World Health Organization Europe. Available from: http://www.euro.who.int/__data/assets/pdf_file/0008/88613/E91438.pdf.

23. Atun R, Menabde N. Health systems and systems thinking. In: Coker R, Atun R, McKee M, editors. Health Systems and the Challenge of Communicable Disease: Experiences from Europe and Latin America. European Observatory on Health Systems and Policies Series. Berkshire, England: Open University Press; 2008. p. 121-40.

24. Kelley E, Hurst J. Health Care Quality Indicators Project Conceptual Framework Paper. Paris: Organisation for Economic Co-operation and Development; 2006. Available from: http://www.oecd.org/ els/health-systems/36262363.pdf.

25. Roberts M, Hsiao W, Berman P, Reich M. Getting Health Reform Right: A Guide to Improving Performance and Equity: Oxford University Press; 2004. 332 p.

26. Shakarishvili G, Lansang MA, Mitta V, Bornemisza O, Blakley $M$, Kley N, et al. Health systems strengthening: a common classification and framework for investment analysis. Health policy and planning. 2011 Jul;26(4):316-26. PubMed PMID: 20952397. Pubmed Central PMCID: 3118911.

27. The Commonwealth Fund Commission on a High Performance Health System. Framework for a High Performance Health System for the United States: The Commonwealth Fund; 2006. Available from: http://www.commonwealthfund.org/ $/$ media/files/publications/fundreport/2006/aug/framework-for-a-high-performance-health-system-for-the-unitedstates/commission_framework_high_performance_943-pdf.pdf.

28. Sub-Saharan Africa: From Crisis to Sustainable Growth. Washington, D.C.: World Bank; 1989.

29. Health for all in the twenty-first century. Revista Panamericana de Salud Pública. 1998;4:132-41 (condensed from WHO Document A51/5, Health for all in the twenty-first century. Geneva, World Health Organization, 1998).

30. Health21: the health for all policy framework for the WHO European Region. Copenhagen: World Health Organization Regional Office for Europe; 1999.

31. Barbazza E, Tello JE. A review of health governance: Definitions, dimensions and tools to govern. Health Policy. 2014 5//;116(1):1-11.

32. Kickbusch I, Gleicher D. Governance for health in the 21st century Copenhagen: World Health Organization Regional Office for Europe; 2012. 128 p.

33. Adelaide Statement on Health in All Policies: moving towards a shared governance for health and well-being - Adelaide: World Health Organization, Government of South Australia; 2010. Available from: http://www.who.int/social_determinants/hiap_statement_who_sa_final.pdf.

34. UCL Institute of Health Equity. Review of social determinants and the health divide in the WHO European Region: final report. Copenhagen: World Health Organization Regional Office for Europe; 2013. Available from: http://www.instituteofhealthequity.org/projects/who-europeanreview.

35. Dodgson R, Lee K, Drager N. Global Health Governance - A Conceptual Review: Centre on Global Change \& Health, London School of Hygiene \& Tropical Medicine; Dept of Health \& Development, World Health Organization; 2002. Available from: http://whqlibdoc.who.int/publications/2002/a85727_eng.pdf. 
36. Londoño J-L, Frenk J. Structured pluralism: towards an innovative model for health system reform in Latin America1. Health Policy. 1997 7//;41(1):1-36.

37. "Roberts, J.L. Terminology for the WHO Conference on European Health Care Reform. Copenhagen, WHO Regional Office for Europe, 1996." In: Health21: the health for all policy framework for the WHO European Region. Copenhagen: World Health Organization Regional Office for Europe; 1999.

38. Whitehead M. The concepts and principles of equity and health (EUR/ICP/RPD 414, 7734r). Copenhagen: World Health Organization Regional Office for Europe; 1990.

39. Fitzpatrick M, Bonnefoy X. Environmental Health Services in Europe 4: Guidance on the Development of Educational and Training Curricula. Copenhagen: WHO Regional Office for Europe; 1999.

40. Declaration of Alma-Ata - International Conference on Primary Health Care, Alma-Ata, USSR, 6-12 September 1978: World Health Organization. Available from: http://www.who.int/publications/almaata_declaration_en.pdf.

41. Western Pacific regional strategy for health systems based on the values of primary health care: World Health Organization, Western Pacific Region; 2010.

42. Primary health care - Main terms used: WHO Europe web site; [cited 2012 27.08.2012]. Available from: http://www.euro.who.int/en/what-we-do/health-topics/Health-systems/primaryhealth-care/main-terms-used. 
Life is difficult. This is a great truth, one of the greatest truth... Once we truly know that life is difficult-once we truly understand and accept it-then life is no longer difficult. Because once it is accepted, the fact that life is difficult no longer matters.

M. Scott Peck 


\section{Chapter 3}

\section{Looking at the}

U.S. health system from

European perspective

B. Serdar Savas and Tomris Cesuroglu

Health for America: European Arguments for a Paradigm Shift. Abridged version of the chapter is published in Shah A, Colombano J, editors. Learning from the World: New Ideas to Redevelop America Palgrave Macmillan; 2013. p. 167-84. 
Abridged version of this chapter was published as Savas BS, Cesuroglu D. Health for America: European Arguments for a Paradigm Shift. In Shah A, Colombano J, editors. Learning from the World: New Ideas to Redevelop America, Palgrave Macmillan; 2013. p. 169-86.

In the course of the editorial process of the above mentioned book, this chapter was abridged by taking out the two sections that explained the shortcomings of the US system in depth, in particular in relation to broader policy values. In this dissertation, these sections are added in the form of two boxes, as noted also in the text. 


\section{Introduction}

The United States is faced with a major ethical responsibility in health. While it is the world's largest economy, with by far the most powerful army. the level of health of its citizenry is relatively low, out of line with its income and development level.

The health status of the US populace is one of the worst among countries in the Organisation for Economic Co-operation and Development (OECD), as shown by life expectancy, mortality, and morbidity indicators. The US ranks 27 within its peer group of 34 OECD countries for life expectancy at birth $(78,2$ years for the total population in 2009) (see Figure 1). The infant mortality rate was 6,5 deaths per 1.000 live births in 2008 , well above the OECD average of 4,4 in 2009. Obesity is at an alarming rate for adults and children, with $34 \%$ of the adult population classified as obese and $32 \%$ of children classified as obese or overweight in 2008. These levels are the highest among OECD countries.

Given these health indicators, it is interesting to note that the US is by far the highest spender on healthcare among OECD countries (see Figure 2), both in terms of the percentage of gross domestic product $(17 \%)$ and per capita expenditure $(\$ 7.960)$ devoted to health. This per capita number is 2,5 times more than the OECD average of $\$ 3.223$.

Unlike most OECD countries, where health spending is mainly financed by the government out of taxes or social security contributions, government spending in the US accounts only for half of total health expenditure. That said, the total level of health spending in the US is so high that per capita public (i.e., government) spending on health is greater than in all other OECD countries, except for Norway and the Netherlands. Despite this high level of expenditure, the US government provides coverage only for a limited group of people, which includes the elderly and disabled (through Medicare) and a portion of the poor (through Medicaid and the State Children's Health Insurance Program). This per capita amount would be enough for most OECD country governments to provide universal health insurance coverage.

The high expenditure and low health outcomes show that the US health system is ineffective. The country's wealth is not translated into gains in health.

The US system establishes a strong basis for an inequitable distribution of health. There are more than 50 million uninsured people in the U.S. (17\% of the population in 2011) who are not provided health insurance by an employer and are unable to afford, cannot qualify for, or choose not to purchase health insurance. When healthcare services through charity are not available, they simply do not get the medical care and treatment they need. Furthermore, healthcare costs create a significant burden even for insured families. It is estimated that around one-fifth of the insured population is actually 'underinsured'. 


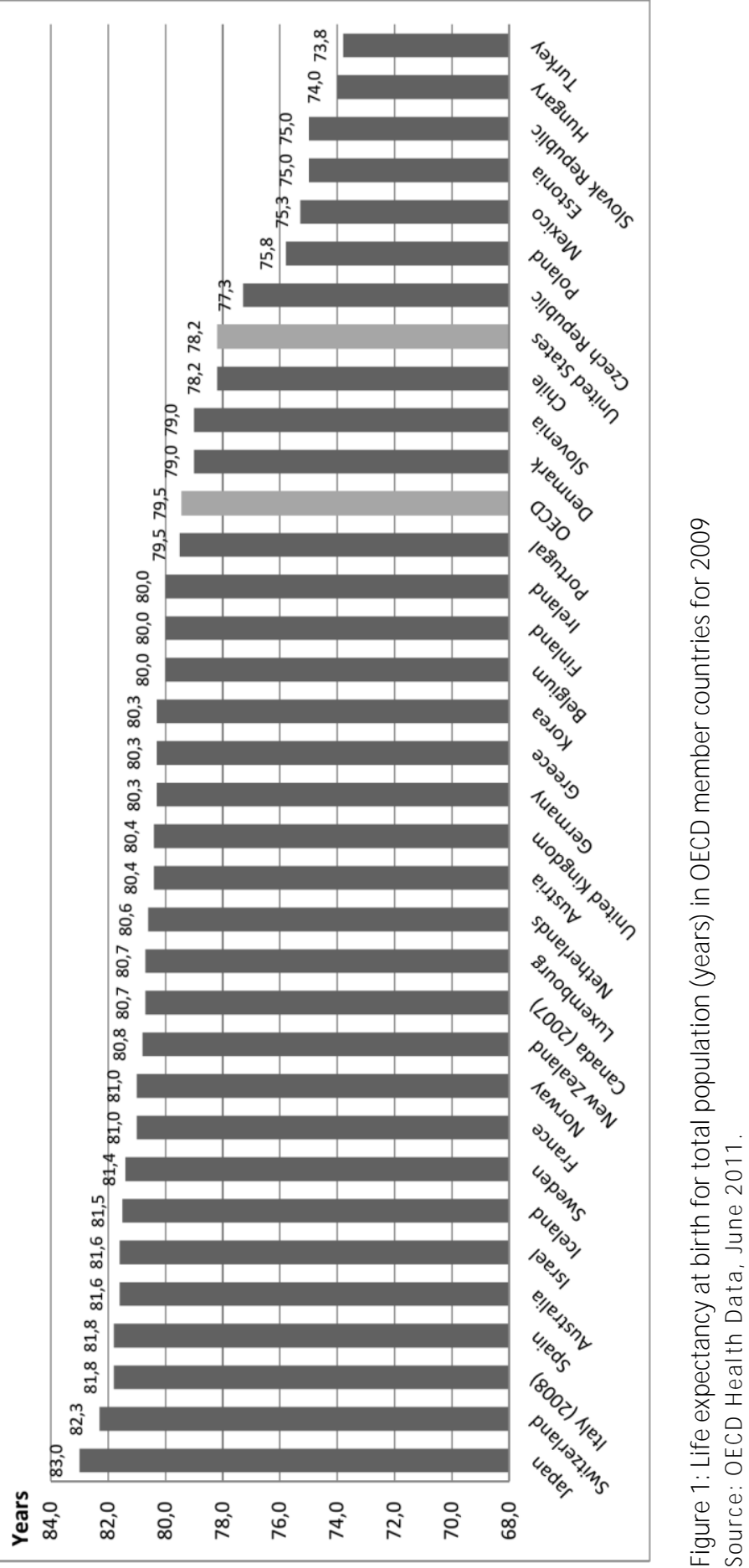




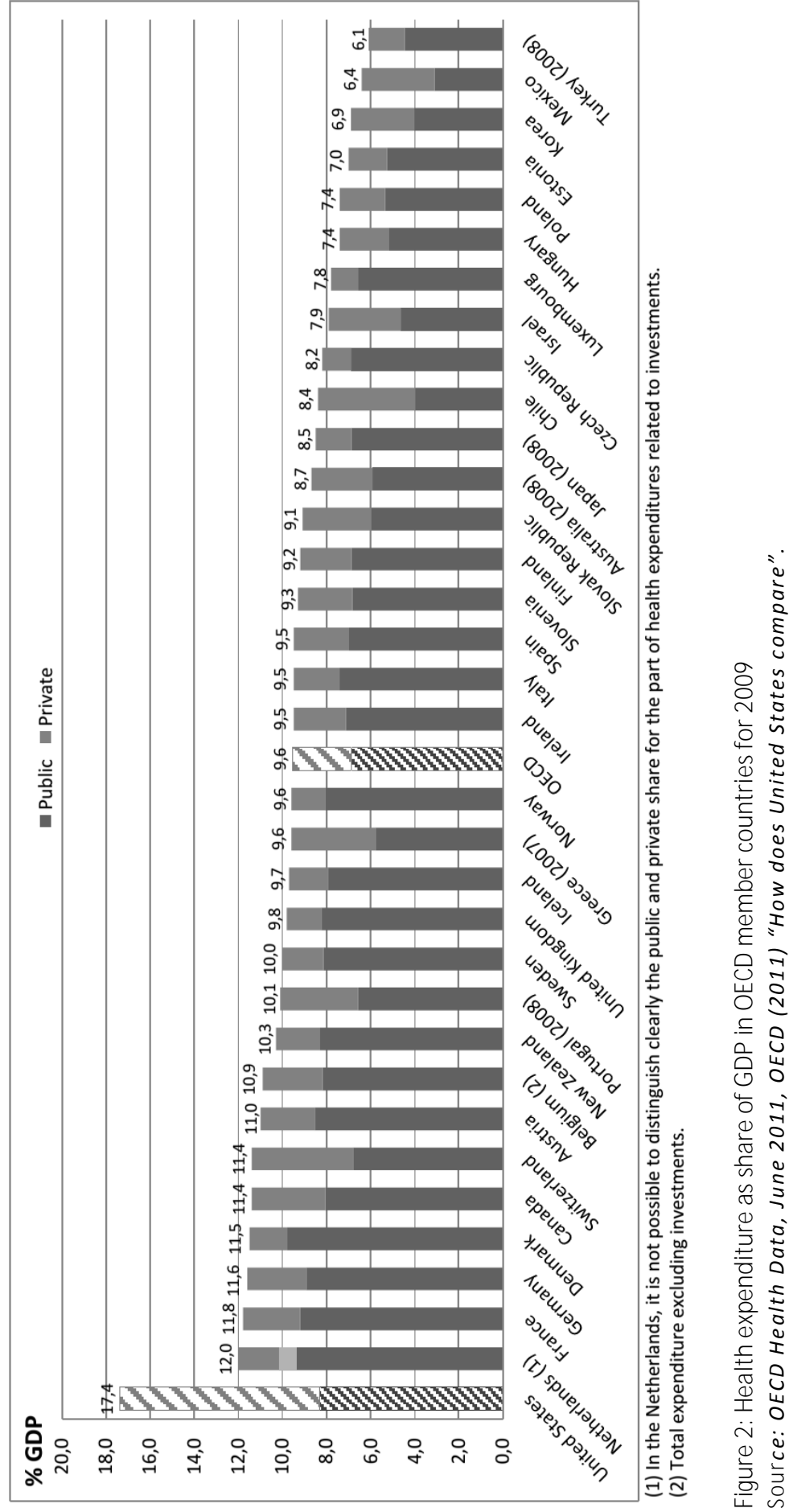


Such people have some form of health insurance, but face significant cost-sharing provisions or limits on benefits. These factors render their insurance useless in paying for needed health services. This is evidenced by the fact that, in 2007 , nearly $80 \%$ of bankruptcies linked to medical expenses (which formed $62 \%$ of all bankruptcies) were filed by people who had health insurance.

The system also hosts disparities in access to health and health status. Even more striking than the percentage of uninsured people is the fact that $10 \%$ of children under 18 are without any coverage, neither public nor private. For children in poverty, this rate increases to $15 \%$. Indicators also show important disparities in health for disadvantaged populations, such as minorities, women, children, and the elderly. For example, Hispanic/Latino Americans and African Americans, and especially their children, are much more affected by the obesity epidemic.

The system causes great inefficiencies owing to various factors, including payment systems that reward inputs, processes, and outputs, rather than actual health outcomes; high administrative costs; and an inadequate focus on disease prevention.

The US healthcare industry's administrative costs are strikingly high. Health administration costs in 1999 were $\$ 1.000$ per capita in the US as compared with $\$ 300$ in Canada. Such costs flow through the system to affect the entire range of actors, including insurance companies, healthcare providers, and pharmaceutical, diagnostic, and medical supplies industries. Additional administrative costs accrue from several factors, including the bureaucracy surrounding the filing of insurance claims, the bureaucracy necessary to ensure compliance with state and federal regulations, lobbying and marketing, and the malpractice insurance system for healthcare professionals-mainly doctors and healthcare facilities. The direct costs of malpractice litigation represent a small fraction of total healthcare costs $(0,46 \%)$. 'Defensive medicine' practices, however, which are characterized by the increased utilization of tests and procedures by physicians to protect against future lawsuits, create the real financial burden on the system. These are estimated by some critics to represent $5-9 \%$ of total health expenditure.

This chapter proposes solutions to problems of the US health system. The solutions we propose draw heavily on the experiences of European countries. We emphasize practical policy choices over academic concerns. While there are also positive properties of the current state of the healthcare sector in the US, some of which are superior to other industrialized countries, for the sake of brevity these will not be covered here. 


\section{Box 1: The main problems of the American Health System ${ }^{1}$}

It is possible to group the problems of America's health system under three major headings:

\section{America does not have a health policy}

For many years, health literature emphasized models that viewed the determinants of disease through a biological framework. During this period, health was supposed to be derived through medical care. This approach was transformed in the 1970 s, as a result of studies that demonstrated the importance of socio-economic, environmental and behavioral factors on health outcomes.

The resulting enlarged set of the determinants of health are shown in Figure 3:

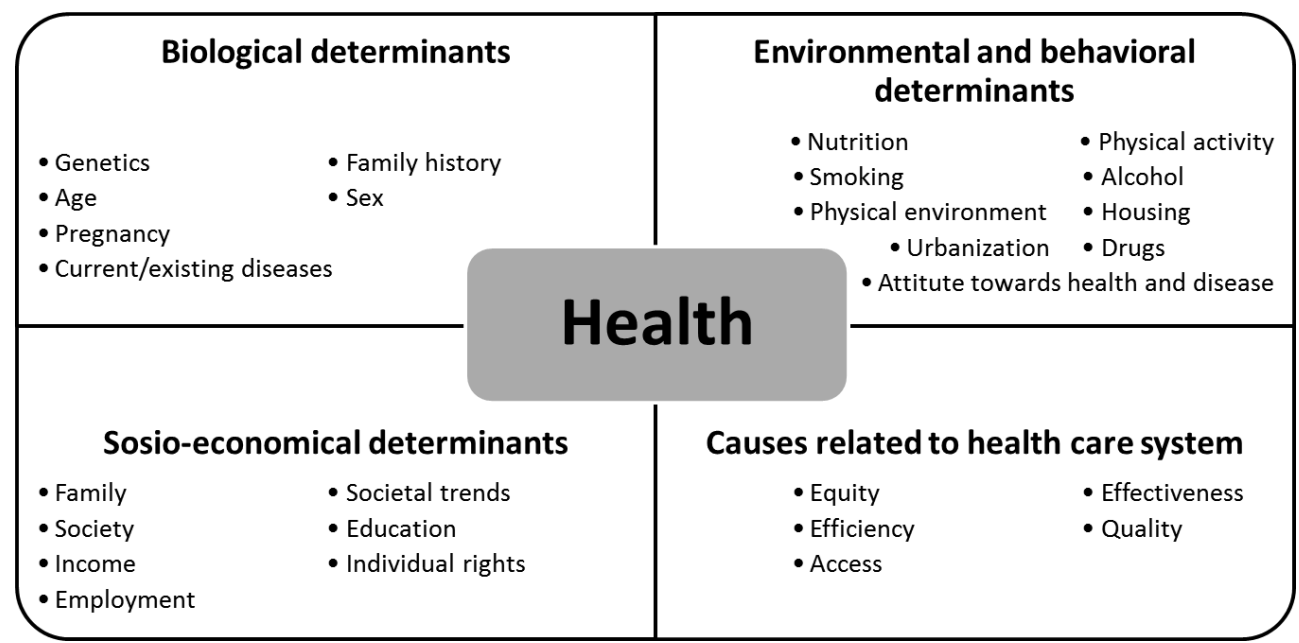

Figure 3: Determinants of health and disease

As seen above, there are four main factors, each with several sub-components that interact to determine an individual's health. A country's health policies must be formulated to address all of these factors.

Yet, discussions around 'health policy' or the formation of a 'health system' in the U.S. are centered solely on the provision of 'health care', just one of the four determinants of health. While it is an important determinant, it cannot be the sole component of a comprehensive system. As such, the U.S. does not have a health policy that leads the nations

\footnotetext{
${ }^{1}$ The section where we explained the shortcomings of the U.S. system in relation to broader policy values was taken out in the course of the editorial process of the publication. To maintain coherence within this dissertation, this material is put back in the form of a box in this chapter.
} 
towards defined health outcomes, based on a thorough engagement of all determinants of health.

\section{America does not have a 'health system'}

Every sub-component of what could be called 'health care' in America developed individually and independently, in an organic manner that responded to certain historical realities. Various health care stakeholders have shaped policy and practice through the years, in accordance with their own desired outcomes and expectations, through lobbying and the targeted shaping of public opinion. Rather than aiming for a healthier nation, the resulting structure is one that serves the interests of a diverse group of stakeholders. In short, there is no health care structure that aims to improve the nation's health.

Debates around 'health problems in USA' are centered on financial issues such as the rising costs of health care and shortfalls in insurance coverage. Barack Obama's 'health reform' is the latest attempt to address these issues. This package falls far short of comprising a 'reform'; it is only another incrementalist effort towards improving health care financing, access, coverage and costs.

However, health care has parts other than financing, such as service delivery and stewardship. America does not have a values-based health system that addresses the population's needs, unites all of these parts, and defines the functions of and relations between the various sub-parts in a consistent manner.

In its simplest form, a 'health system' is composed of four main parts (Figure 4).

'Users' in USA are divided between several sub-groups. Among the insured, there are those insured through their employers or service sectors, those that receive insurance as a result of their age or gender, those that pay for insurance out of pocket, and others that fall into insurance categories as a result of their retirement status or other reasons. Each policy has its idiosyncrasies, with different premium payment features and benefits. There are also people who are not insured. Individuals' benefit packages are not shaped by their needs, but are rather formed by their initial level of health and the amount of their premium payments. This is one reason why the American health system creates inequity.

There is also a wide range of institutions on the 'payer' side. There are those that are not for profit (government schemes such as Medicare and Medicaid) as well as for-profit private insurance companies. Each payer institution has its own internal mechanisms. In general, payers tend to avoid cost-effective interventions that incur costs in the present, but prevent disease and subsequent complications in the future, increase quality of life and prolong life-span. Rather, they choose to pay for the interventions that will save money today and in the short run, but that have continued to result in ever-increasing long term costs. Recent legislative changes made under the presidency of Barack Obama man- 
date ceilings on administrative costs. Yet, instead of actually lowering these costs, these legislative changes are bound to lead to their re-classification, showing up as increased costs elsewhere. Such administrative costs, combined with the profit motive of many payers, result in upward pressure on insurance premiums.

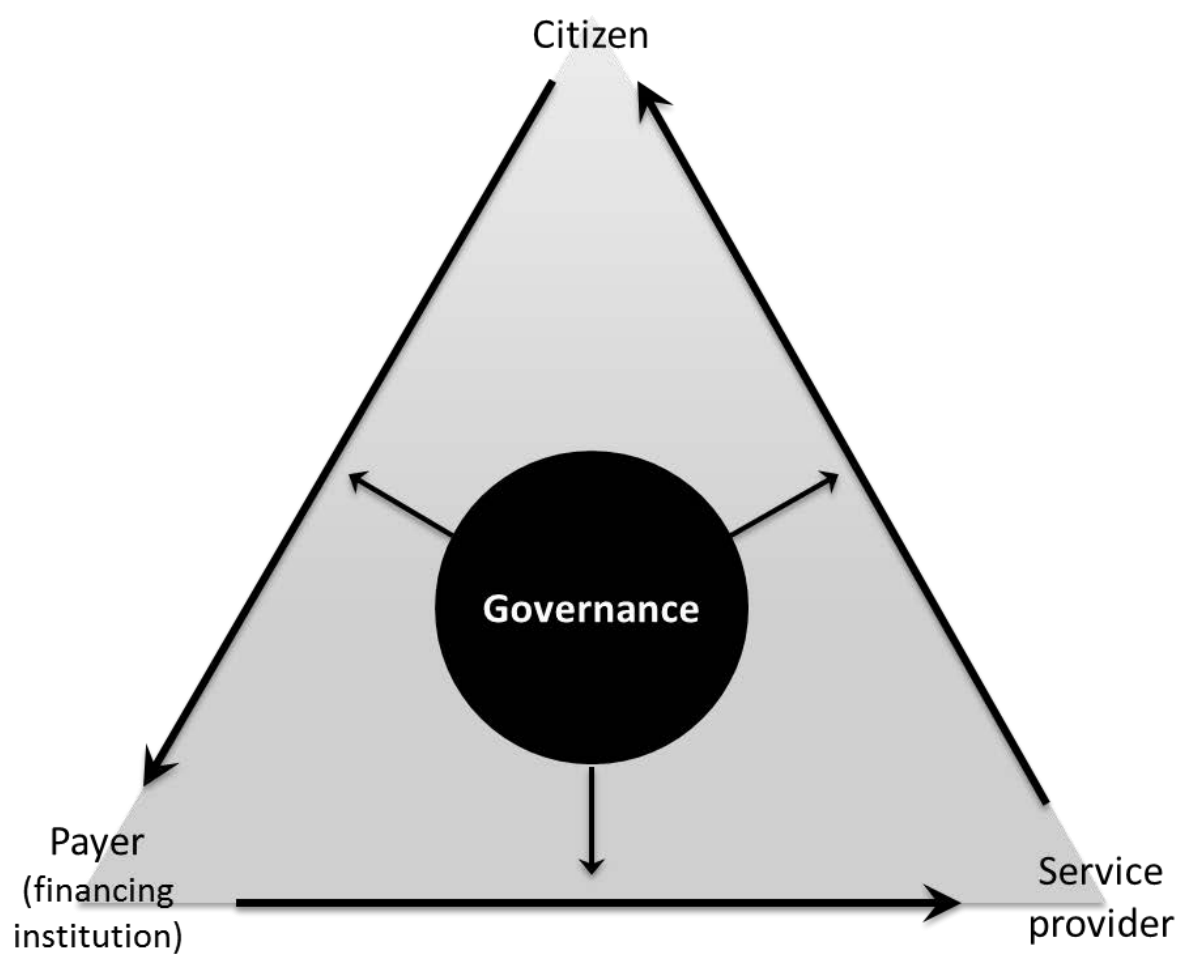

Figure 4: Main parts that constitute a health system

The service provider landscape is similar. It spans public and private, for-profit and nonprofit institutions, without a standardization of functions. Access to primary health care provision is limited, and it can be said that U.S. medical staff are not trained in line with any overarching policy goal. Complex governance systems, profit motives, malpractice insurance and the subsequent tendency for defensive medicine are factors that keep costs high on the health service provider side.

The three parts (Citizens - Payers - Providers) are steered and governed by a complex web of legislation and regulations, the organizational and management perspectives of which are disjoint and irregular. The stewardship and governance of the U.S. health care sector is not premised on common goals and objectives.

The absence of a unified and purposeful health system in USA stems from a belief that health is a commodity that can be allocated efficiently and effectively through a free- 
market mechanism. This view is advocated primarily by stakeholders who benefit from the current system, and discussions relating to the sector consistently feature a misuse of concepts, which is often deliberate.

As a result, the U.S. health system does not aim to achieve a higher, more equally distributed level of health for its citizens. It does not possess a body of values that would direct health initiatives towards areas that would lead to the largest improvements in health. Furthermore, instead of working in harmony in a synergistic manner, the various parts of the health system display tension and dissonance.

\section{The health care debate in America is conducted on ideological grounds, not based on sound economic theory}

An overview of the health care debate in America reveals an ideological approach, as opposed to an economic one. It is continuously claimed that the most appropriate method for the provision of health services is through the rules of a free market economy, that a different approach would constitute socialized medicine, that this would be the implementation of a socialist / communist system, and that this would therefore lead to lower efficiency and quality.

Yet, if one were to leave ideological prejudice aside, one would see, from a scientific perspective, that health economics has much to say on these issues. Our intention within the limited scope of this paper is not to present facts that could be found in any health economics textbook. It is important, nonetheless, to underscore a few points of importance in order to provide a background for our approach.

There is a significant information imbalance between the producers (especially physicians) and the consumers (citizen / patient) of health care. A patient approaches a physician with a complaint. The patient has a need, but this need has not yet been translated into demand in the economic sense. Following an examination and further diagnostic procedures, the physician makes a decision on what should be done with the patient. There is an agency relationship between the patient and the physician. The physician's decision forms the patient's demand. This is called supplier induced demand. Research shows that the demand for treatment induced by the physician is not always for the benefit of the patient. $A$ consideration by the physician of his or her own interests can be a factor that plays into the recommended course of action. This informational asymmetry means that there is limited transparency in a patient - physician relationship. Since transparency is a precondition for the efficient functioning of a free market, supply and demand cannot interact freely under such conditions.

Health care also results in certain externalities, especially in the case of infectious diseases. The vaccination of a child to protect her from an infectious disease reduces the chanc- 
es of her friends catching that disease.

In addition to this, the non-excludable nature of certain health services means that health care is a public good: it is not always possible to prevent people who have not paid for it from benefiting from it. For example, in the fight against malaria, once a drainage, sanitation or spraying effort has been undertaken, it is not possible to tell the remaining mosquitoes to only bite those people that have not paid for these services.

The provision of health services features significant barriers to market entry, especially for physicians. Anyone can sell a car, a television or a vacation, but not anyone can sell diagnostic and treatment services. A country can import rice and textiles, but not health services. Medical associations, not only in America but throughout the world, endeavor to erect barriers to entry for others in an effort to protect the interests of their professional membership. There are also many instances of barriers to entry in the market for insurance, leading to certain regional insurance monopolies.

Monopolies also exist in the pharmaceutical sector, where pharmaceutical companies are granted immunity from competition through legal arrangements such as patents and data protection, so that they can profit from their significant investments in research and development.

M ost of the factors listed above lead to price increases and the implementation of treatments that are harmful or the health benefits of which are limited. Furthermore, in a free market system where health care is purchased through out-of-pocket expenditure, citizens without the ability to pay cannot afford health care, leading to serious health inequities.

The above is not an exhaustive list of arguments for why health care cannot be treated as a free market commodity. A survey of health economics literature will yield other arguments, in addition to further detail on those listed above.

For years, the main beneficiaries of the status quo of the American health system have been groups that have benefited from the market failures outlined above. As such, these groups deliberately refrain from using scientific or economic arguments, and employ ideological terminology that prevents the formation of a healthy, facts-based debate. 


\section{Box 2: Values and principles that should guide establishment of American Health System ${ }^{2}$}

America needs a comprehensive, holistic, rational change in its understanding of 'health policies' and 'health systems'. This requires a fundamental paradigm shift in the political, social and economic dimensions of health, challenging certain prevailing presumptions in American politics. Such a transformation cannot be implemented through one, two or three presidential terms. It requires a state policy that will manage change for several decades. This paradigm shift can be affected by developing an American Health Policy and establishing an American Health System based on certain values and principles.

There are several values and principles inherent in many European health policies, which would be required for such a paradigm shift in America:

1. Health is a basic human right

2. Health is the complex result of many biological, social, economic, environmental, behavioral and health care related factors, and cannot be reduced to 'health care' alone.

3. Health can be improved through a concerted effort undertaken by society as a whole

4. Health policies must be long term and supra-political

5. A health system should be based on the following combination of values:
a. Health gain
b. Solidarity
C. Equity
d. Effectiveness
e. Efficiency

6. Factors such as externalities and the vast information asymmetries between users and providers / payers mean that a free market approach to health care will result in significant shortcomings

7. Health is a public good

There is a discourse in USA that embraces these values and principles and incorporates suggestions that would take America towards such a values-based approach. 'The Future of Public's Health in the 21st Century' report of the Institute of Medicine (2002) can be

\footnotetext{
${ }^{2}$ The section where we summarized the recommended policy values and principles was taken out in the course of the editorial process of the publication. To maintain coherence within this dissertation, this material is put back in the form of another box in this chapter.
} 
cited as one such example. Despite thorough and thoughtful studies and recommendations put forward by these commissions, this fundamental approach and its accompanying system of values have not been able to penetrate into the health sector. As a result of the absence of a unified health system, discussions relating to public health take place in a vacuum that remains separated from the health services sector.

\section{Developing an 'American Health Policy'}

An American Health Policy must address all the determinants of health. It should not be legally binding. It should aim to be a guiding document that draws a framework for future governments, decision makers, managers, health professionals, and other related industry participants. Such a document should aim to declare a 'national commitment' to improving the health of the American nation. As a result, such a policy must incorporate rational and measurable long-term goals for mortality and morbidity.

For example, the policy could set a target for "reducing deaths from coronary heart disease in the US by $20 \%$ by 2040". Such a target would necessitate changes in socioeconomic policies, individuals' behaviors, and environmental factors, in addition to changes in the provision of healthcare. These changes would include a range of efforts, such as the prevention of obesity, the promotion of healthy eating habits and exercise, campaigns against tobacco use, the taxation of certain 'public bads', the identification of risks for coronary heart disease in the population and its sub-groups, the establishment of risk control systems, an analysis of the most cost-effective treatment methods, and the development of early diagnostics and timely intervention methods. Only a small proportion of these activities relate to the healthcare sector. The majority involves areas ranging from education to agriculture to labor policies, from taxation to inner-city traffic controls and athletics. The American Health Policy must establish a platform for all of these activities. It must further ensure that the health impacts of non-health policies (e.g., construction, transportation, energy policies) are considered and evaluated at all times using health impact assessment tools.

Targets must be established in a similar fashion for all matters that lead to loss of health and life, from diabetes to osteoporosis, from asthma to traffic accidents. The necessary activities relating to each such field must be identified through a participatory, multisectoral process, as in the example of coronary heart disease above.

In general, the American Health System must aim for the maximum possible level of health for its citizens. As explained above, this will require explicit commitments to reach predefined targets within a certain timeframe. Ideally, targets should be 'SMART': Specific, Measurable, Accurate, Realistic, and Time-bound. 
Successful target setting requires a thorough understanding of health and disease and the ability to measure the effectiveness of health interventions, as well as epidemiological, demographics, and modeling expertise. The US is not short on such skills.

We argue that a policy for health extends far beyond 'healthcare'. As such, the responsibility for developing such policies should not rest with healthcare professionals, but should be the task of an inter-sectoral body formed under the auspices of the federal government. This body should include members of the government, the private sector, and society at large. As it conducts its works at the federal level, similar state commissions should be formed to perform the same task at the state level. Inconsistencies between federal and state policies must then be resolved in order to create nationwide harmony in policy goals and targets.

Our coronary heart disease example set 2040 for its targeted completion date. Yet it is important to establish interim targets and milestones while pursuing long-term policies. M onitoring bodies at the federal and state levels must follow and evaluate developments. Furthermore, the development of an American Health Policy must be accompanied by an effective communication strategy.

The development of a national health policy in Finland is a good example for such a comprehensive effort, and can serve as a useful case study. The Health 2020 document that has recently been developed by the World Health Organization's Regional Office for Europe can also provide an inspiring framework.

\section{Developing an American Healthcare System}

For an American Health Policy to reach its goals, and for healthcare in America to develop into a congruent system, the structure and function of its parts must be defined, with the aim that these parts are made to work together as a whole.

Healthcare must become preventive, predictive, personalized, and participatory. A 'one size fits all' approach is no longer viable. Developments in genomics and system biology further mandate the need for older practices to give way to new ones. While designing the American Healthcare System, this vision of the future, its possibilities, and its prerequisites must be taken into consideration.

\section{The system's users}

In its simplest form, a 'health system' is composed of four main parts: the system's users (citizen), payer (financing institution), service providers, and governance. It is the basic human right of every American citizen to access health services in accordance with his or her needs. This is the model for almost all European countries. Financial, physical, social, and economic barriers in the way of access must be removed. While all barriers must be 
tackled, the most pressing obstacles are financial. Those who are most in need of healthcare are also individuals of lower socioeconomic standing. However, since risks are individually determined in the American insurance model, these individuals have to pay higher premiums for healthcare. Insurance companies have also been allowed to keep high-cost individuals out of the system. Bans and regulations cannot prevent these practices entirely as long as the system is profit-oriented; different methods of exclusion will continue to develop.

\section{The payers' perspective}

Healthcare financing must be organized such that it fosters social cooperation and solidarity. Within this model the healthy will support the sick, the young will support the elderly, and the rich will support the poor.

A comprehensive financing system must begin with the establishment of an insurance pool that covers every citizen. In general, bigger insurance pools are better able to provide coverage for catastrophic expenses. Universal coverage is a standard characteristic of European healthcare systems. These can be grouped into two main categories.

The first is the Beveridge model, which originated in the United Kingdom. It is used by a number of European countries, including the United Kingdom (through the National Health Service) and the Scandinavian countries. Under this system, all health expenses are paid by the government through the public budget. Taxpayers do not pay a supplemental premium for healthcare.

The second is the Bismarck model, which originated in Germany and whose variants developed in countries in continental Europe. This is a health insurance system of a public nature. Individuals, with the help of employees, transfer a portion of their salaries to insurance funds as a type of earmarked tax. As such, citizens pay a health premium alongside their taxes.

On both models, the premium or tax that an individual pays correlates to the individual's income level. A poor, sick individual, who would have paid a higher premium for health insurance in America, would, under both systems, pay less than a healthy individual with high income. This is where the social solidarity of the European models becomes apparent.

The health system that has been developed in Turkey over recent years is an example worth analyzing. The Turkish Healthcare Reform was developed in the 1990s and implemented in the 2000s. At the time of its development, a key topic of debate was the choice of financing through general taxes or through a separate health insurance contribution. 
Tax revenues in Turkey are primarily composed of taxes deducted from the incomes of salaried workers, and indirect value added taxes (VATs), which are paid at the same level regardless of income. Income taxes and corporate taxes contribute much less to the tax pool than in other European countries or in the US, as a result of an ineffective tax collections mechanism. Financing health expenses through taxes would therefore result in the financing of the system by lower income groups. Turkey has chosen a general health insurance model and tries to ensure equity through premiums linked to an individual's level of income. In this system, premiums of individuals below a certain income level are paid by the state. The inequality that would result from financing healthcare through taxes was compared with the extra costs that would result from the need to collect additional premiums, and a choice was made in favor of the latter.

Conditions in the US are significantly different from those in Turkey. America has an effective tax collection system. Tax receipts are composed primarily of income and corporate taxes, and the level of taxation is, for the most part, directly correlated with income, leading to a relatively just tax structure. As such, leveraging the pre-existing tax system to cover health expenditure would be a more equitable approach to financing healthcare. A co-payment requirement might be considered, once again in line with an individual's ability to pay. Those without an ability to pay would be exempt from such a requirement. The collection of additional health premiums would also create an additional expense item in the US. Therefore, paying for healthcare through general tax receipts would not only be more equitable but would also keep costs lower by avoiding expenses associated with the collecting of premiums.

Every state should allocate resources from its tax pool for healthcare. Additionally, some funds may be transferred to states from the federal budget to provide nationwide equity. Regarding the use of resources by individual states, we suggest the creation of 'Geographic Purchasing Units' (GPUs) for every million citizens. These units would purchase health services from providers based on the health indicators, primary health problems, age and gender distributions, and ethnicities prevailing in their areas. These purchasing units must also be skilled in public health, epidemiology, statistics, economic evaluations of healthcare, health services planning, health service quality, contracting, and contract management.

\section{The reorganization of healthcare providers}

Owing to the introduction of new technologies, the distinction between primary, secondary, and tertiary care levels is not as strong as it was 30 years ago. However, these concepts are of particular importance for the US. All individuals should be analyzed for risks. Precautions should be taken for high-risk individuals, especially those with chronic or complex risks, such as heart attack, stroke, diabetes, cancers, or depression (risk management). For individuals with developed diseases, complications, and other co- 
morbidities, they should be prevented through secondary prevention (disease management). In severe cases where co-morbidity has developed, tertiary prevention (case management) should be applied.

We recommend a three-level structure that consists of first-level healthcare, outpatient specialist care, and inpatient specialist care (hospital care). First- level healthcare should be positioned at the center of the decision-making mechanism, taking the driver's seat. It would consist of family physicians or general practitioners, supported by nurses, midwives (in Holland, for example, around $30 \%$ of deliveries are midwife-led home deliveries), technicians, and administrative staff, and would be the first stop for all individuals, with the exception of emergencies. First-level healthcare providers (also referred to as primary care services) should assume a gatekeeper role. Pediatricians and gynecologists should be no exception.

Based on the experiences of countries such as the UK, Denmark, Iceland, Finland, Norway, and Sweden, it is expected that an efficiently organized first-level healthcare unit will be able to treat at least $80 \%$ of all cases. First-level healthcare units should be responsible for meeting outcome-based performance goals set with regard to the population they serve. These units should have responsibilities in primary, secondary, and tertiary prevention areas, and they should be responsible for ensuring continuation of care. First- level healthcare units must employ emerging information, genetics, diagnostic, and treatment technologies through a 'point of care' approach. These units can bring together public and private elements. Such decisions should be taken based not on ideological factors but on a case-by-case basis for each unit.

A careful forecast of the needs of physicians and nurses must be modeled when transitioning to such a system. This model must account for population and workload distribution, and should feed into further plans regarding the formation of training processes that will develop the required skills and knowledge base.

Outpatient and inpatient specialist care could be purchased from public or private sources. What is important is that these providers can fulfill the services at the prices determined by the GPUs in their areas. Competition will play a role for reducing prices and increasing health outcomes, and will be critical in ensuring that the American Healthcare System meets its targets.

In the new system, health outcomes must feature into providers' compensation schemes. Current payment models (fee for service, diagnosis-related groups, cost and volume, and others) are based on inputs, processes, and outputs. The quality evaluation of providers is also based on an inputs-processes- outputs triangle. There is a false perception that outputs from high-quality inputs and processes will produce good outcomes. For this reason, quality and certification services in the US are limited in their abilities to increase health. 
As in the Danish example, the indicators of providers with regard to health outcomes should be compared to determine the providers with the best health results. By creating databases, every clinic and doctor will be able to compare their results with others. These databases should be anonymous, since the goal is not to create a system of rewards and penalties but, rather, to generate better health outcomes.

Current malpractice regulations should also be addressed. These regulations create a financial burden on the system and on patients because of excessive insurance expenses that add to costs. Malpractice compensation is also seen as a source of income for some patients, and is a profitable field for lawyers and insurance companies. In short, financial compensation leads, ultimately, to behavior that increases the system's costs. Therefore, rather than wealth-producing compensation for patients, malpractice regulations should be directed toward professional development. These regulations should, therefore, be set by professional bodies within an ethical and scientific framework, and should not employ tools that create a financial burden.

\section{Managing Change}

The organizational and systems changes recommended above are complicated and difficult to undertake. This chapter proposes a paradigm shift that is hard for many stakeholders to accept. Heading the list of these stakeholders are medical professionals, private forprofit insurers, private for-profit providers, pharmaceutical companies, and medical device manufacturers. As in the past, these groups will advance the following arguments:

1. The American health system is the best in the world and does not need big structural changes.

2. The US is a world leader in medical practices.

3. The free market is a magic bullet for every problem.

4. The professional freedom of physicians is indispensable.

5. Citizens should make their own decisions.

6. Citizens should have the right to choose.

7. Proposed structural changes will cause job losses in the health sector.

The first six arguments are ideologically driven and are crafted to serve the interests of the groups listed above. In the past, such initiatives to change the system have been called 'socialized medicine' in an effort to press the American public's 'hot buttons'. As for the last argument, while job loss will be encountered within unnecessary structures and functions, these losses will be countered with employment gains necessitated by new structures and functions. 
It is not adequate to simply make the right arguments; the manner in which they are communicated is also important. The development of the American Health Policy must begin with the formation of an eight-digit annual PR budget and a well-executed campaign. Those who profit from the status quo spend hundreds of millions of dollars on campaigns and lobbying. To counter this force, non-governmental organizations (NGOs) and other civil society groups must be strengthened and supported under an umbrella 'Health for America' campaign.

Transition to the new system should be progressive and tiered. The old system's infrastructure and capacities should be recycled and incorporated to the extent that this is possible. This would also ease some of the resistance toward a new system. For example, Health Maintenance Organizations (HM Os) and private health insurers may be used when creating the GPUs. Providers in the new system will mostly comprise current providers with different, disciplined work methods.

\section{Conclusion}

Chronic and complex diseases are rising at a steady pace around the world, and especially in the US. One need not consult a psychic to see that the American public's health is worsening, that healthcare spending will continue to spiral upward, and that the system will eventually come to a gridlock. It is not a technically difficult task to affect the paradigm shift proposed in this chapter, to create a national health policy and develop a better healthcare system. There is considerable experience and know-how in these fields, especially originating in Europe.

As a result of concerted lobbying efforts by the beneficiaries of the status quo, the US has so far not been able to take radical action for reform and has wasted time with incrementalist, palliative initiatives. The efforts of politicians cannot arrest this trajectory and usher in the change required for a healthier American nation with a functional health system. In addition to political power, this paradigm shift requires people with effective leadership. There are several instances of such transformative change in American history. The task at hand is to demonstrate the leadership not to pursue short-term political gains but to state bravely the actions that need to be taken in order to improve the health of a nation that is so important to the rest of the world. Success will come gradually through the focused and persistent efforts of the leaders of this movement. 


\section{Bibliography}

Administrative costs of health care coverage: American M edical Association; [17.10.2011]. Available from:

http://healthcarereform.procon.org/sourcefiles/administrative_costs_of_health_care_coverag e_ama.pdf.

Anderson KJ. A review of health care reform in the United States and in Alaska. Int J Circumpol Heal. 2010;69(5):424-36.

Baris E, Mollahaliloglu S, Aydin S. Healthcare in Turkey: from laggard to leader. Brit M ed J. 2011;342.

Boyle S. United Kingdom (England): Health system review.: World Health Organization, on behalf of the European Observatory on Health Systems and Policies; 2011. 483 p.

Brubaker LM, Picano E, Breen DJ, Marti-Bonmati L, Semelka RC. Health care systems of developed non-U.S. nations: strengths, weaknesses, and recommendations for the United States-observations from internationally recognized imaging specialists. AJR Am J Roentgenol. 2011;196(1):W30-6.

Busse R, Riesberg A. Health Care Systems in Transition: Germany. Copenhagen: World Health Organization Regional Office for Europe on behalf of the European Observatory on Health Systems and Policies; 2004. 234 p.

Carey D, Herring B, Lenain P. Health Care Reform in the United States: OECD; 2009. Available from: http://www.oecd.org/officialdocuments/publicdisplaydocumentpdf/?doclanguage $=e n \& c o t e=e$ co/wkp(2009)6.

Cassels A. Health Sector Reform: Key Issues in Less Developed Countries. Journal of International Development. 1995;7(3):329-47.

Cinsel Sağlık ve Üreme Sağlığı Ulusal Stratejik Eylem Planı [Sexual and Reproductive Health National Strategic Action Plan for Health Sector, 2005-2015]. Prepared for M inistry of Health- Republic of Turkey, UNPFA. M other and Child Health and Family Planning General Directorate, M inistry of Health, Republic of Turkey; 2005.

Coons S). Health care reform in the United States: so near and yet so far. Clin Ther. 2010;32(3):5445.

Cutler DM . The American Healthcare System. Medical Solutions. 2008 June 2008:20-4.

DeNavas-Walt C, Proctor BD, Smith JC, U.S. Census Bureau. Income, Poverty, and Health Insurance Coverage in the United States: 2009. Washington D.C.: U.S. Census Bureau; 2010. Available from: https://www.census.gov/prod/2010pubs/p60-238.pdf.

Diyabet 2020: Vizyon ve Hedefler - Türkiye [Diabetes 2020: Vision and Targets - Turkey]. Istanbul: Turkish Diabetes Foundation, under the auspices of the Turkish Ministry of Health, in collaboration with World Health Organization Regional Office for Europe and International Diabetes Foundation Europe, 2010.

Docteur E, Oxley H. Health-Care Systems: Lessons from the Reform Experience: OECD; 2003. Available from: http:// www.oecd.org/ els/health-systems/22364122.pdf.

Doherty RB. Universal Health Care Coverage in the United States: Is It "Slip Slidin' Away"? Ann Intern Med. 2011;154(2):118-+.

Donaldson MS, Yordy KD, Lohr KN, Vanselow NA. Primary Care: America's Health in a New Era. Washington D.C.1996. Available from: http:// www.nap.edu/openbook.php?isbn=0309053994.

Dougherty D, Conway PH. The "3T's" road map to transform US health care - The "how" of highquality care. Jama-J Am Med Assoc. 2008;299(19):2319-21. 
Looking at the U.S. health system from European perspective

European Health Care Reforms: Analysis of Current Strategies: World Health Organization Regional Office For $\quad$ Europe; $1996 . \quad$ Available from: http://www.euro.who.int/__data/assets/pdf_file/0005/111011/sumhecareform.pdf.

Fielding JE, Teutsch S, Breslow L. A Framework for Public Health in the United States. Public Health Reviews. 2010;32(1):174-89.

Figueras J, M cKee M, Cain J, Lessof S, editors. Health systems in transition: learning from experience: World Health Organization, on behalf of European Observatory on Health Systems and Policies; 2004.

Figueras J, Robinson R, Jakubowski E, editors. Purchasing to improve health systems performance. Berkshire, England: Open University Press; 2005.

Figueras J, Saltman RB. Building upon comparative experience in health system reform. Eur J Public Health. 1998;8(2):99-101.

Figueras J. Health system reforms and post-modernism - The end of the big ideas. Eur J Public Health. 2003;13(1):79-82.

Frieden TR. A Framework for Public Health Action: The Health Impact Pyramid. Am J Public Health. 2010;100(4):590-5.

Funding health care: options for Europe. M ossialos E, Dixon A, Figueras J, Kutzin J, editors. Buckingham, Philadelphia: Open University Press; 2002.

Galas DJ, Hood L. Systems Biology and Emerging Technologies Will Catalyze the Transition from Reactive M edicine to Predictive, Personalized, Preventive and Participatory (P4) Medicine. Interdisciplinary Bio Central. 2009;1(2):1-4.

Garson A. The US healthcare system 2010 - Problems, principles, and potential solutions. Circulation. 2000;101(16):2015-6.

Garssen J, van der Meulen A. Perinatal mortality in the Netherlands. Backgrounds of a worsening international ranking. Demographic Research. 2004;11:357-94.

Geyman JP. Myths as barriers to health care reform in the United States. Int J Health Serv. 2003;33(2):315-29.

Glenngård AH, Hjalte F, Svensson M, Anell A, Bankauskaite V. Health Systems in Transition: Sweden. Copenhagen: World Health Organization Regional Office for Europe on behalf of the European Observatory on Health Systems and Policies; 2005.

Grob GN. Founding Friends: Families, Staff, and Patients at the Friends Asylum in Early NineteenthCentury Philadelphia. Journal of the History of Medicine and Allied Sciences. 2008;63(3):405-7.

Halldorsson M. Health care systems in transition: Iceland. Copenhagen: World Health Organization Regional Office for Europe on behalf of the European Observatory on Health Systems and Policies; 2003.

Health care reform: what the United States can learn from the experience of other developed nations. Health Serv Res. 2010;45(2):588-601.

Health Insurance Coverage Status and Type of Coverage All Persons by Age and Sex: 1999 to 2009 (Table HIA-2) [Internet]. 2011. Available from: http://www.census.gov/hhes/www/hlthins/ data/historical/footnotes.html.

Health Reform Project, Final Report: Prepared by BSS -United Health Systems for the Ministry of Health, Republic of Turkey \& the World Bank; 2003.

Health Reform Update: American Public Health Association; 2011 [24.06.2011]. Available from: http://www.apha.org/NR/rdonlyres/F188C29D-C39C-4959-97A6-

751017AA4DE9/0/HealthReformUpdateOneYearAnniversary.pdf. 
Health Transformation Program in Turkey, Progress Report: Republic of Turkey, Ministry of Health; 2010. Available from: http://www.saglik.gov.tr/EN/belge/2-387/health-transformationprogramme-in-turkey---progress-re-.html.

Health21: the health for all policy framework for the WHO European Region. Copenhagen: World Health Organization Regional Office for Europe; 1999.

HealthCare.gov Washington, D.C.: Department of Health \& Human Services, USA; 2011 [June 2011]. Available from: www.healthcare.gov.

HealthCare.gov. About the Law (Affordable Care Act) 2010 [24.06.2011]. Available from: http://www.healthcare.gov/law/about/index.html.

HealthCare.gov. About the Law: Provisions of the Affordable Care Act, By Year 2010 [24.06.2011]. Available from: http://www.healthcare.gov/law/about/ order/byyear.html.

Heffler S, Smith S, Keehan S, Borger C, Clemens M K, Truffer C. U.S. health spending projections for 2004-2014. Health affairs. 2005;Suppl Web Exclusives:W5-74-W5-85.

Himmelstein DU, Thorne D, Warren E, Woolhandler S. Medical bankruptcy in the United States, 2007: results of a national study. Am J Med. 2009;122(8):741-6.

Hoffman B. Health care reform and social movements in the United States. Am J Public Health. 2003;93(1):75-85.

Hood L, Friend SH. Predictive, personalized, preventive, participatory (P4) cancer medicine. Nat Rev Clin Oncol. 2011;8(3):184-7.

Hood L. A Doctor's Vision of the Future of Medicine. Newsweek. 2009 Jul 13, 2009.

IOM - Institute of Medicine. Insuring America's Health: Principles and Recommendations. Washington D.C.: The National Academies Press; 2004. Available from: http://iom.nationalacademies.org/en/Reports/2004/Insuring-Americas-Health-Principles-andRecommendations.aspx.

IOM -Institute of M edicine. State of the USA Health Indicators: Letter Report. Washington D.C.: The National Academies Press; 2009.

Jacobs LR, Skocpol T. Health Care Reform and American Politics: What Everyone Needs to Know New York: Oxford University Press, USA; 2010 October 20, 2010.

Johnsen JR. Health Systems in Transition: Norway. Copenhagen: World Health Organization Regional Office for Europe on behalf of the European Observatory on Health Systems and Policies; 2006. $167 \mathrm{p}$.

Kessler DP, McClellan M. Do Doctors Practice Defensive Medicine? [Working Paper]: National Bureau of Economic Research; 1996. Available from: http://www.nber.org/papers/w5466.

Kickbusch I. Old Europe - New Public Health. Prev Chronic Dis. July 2007;4(3):1-3.

Kinney ED. Health Care Financing and Delivery in the United States, M exico, and Canada: Establishing Intentional Principles for Sound Integration. Wisconsin International Law Journal. 2009;26.

Knickman JR, Kovner AR. Overview: The State of Health Care Delivery in the United States. In: Kovner AR, Knickman JR, editors. Jonas and Kovner's health care delivery in the United States. 9 ed. New York: Springer Publishing Company, LLC; 2008.

Leu RE, Rutten FFH, Brouwer W, M atter P, Rütschi C. The Swiss and Dutch Health Insurance Systems: Universal Coverage and Regulated Competitive Insurance Markets: The Commonwealth Fund; 2009. Available from: http://www.commonwealthfund.org/publications/fundreports/2009/jan/the-swiss-and-dutch-health-insurance-systems--universal-coverage-andregulated-competitive-insurance. 
Looking at the U.S. health system from European perspective

Longest BB. Health Policymaking in the United States. 5th ed: Health Administration Press; 2010 M ay $15,2010$.

Manchikanti L. Health Care Reform in the United States: Radical Surgery Needed Now More Than Ever. Pain Physician. 2008;11(1):13-42.

McCanne DR. The Organization for Economic Cooperation and Development and Health Care Reform in the United States. Int J Health Serv. 2009;39(4):699-704.

McClellan MB. Testimony of Mark B. McClellan, MD, PhD Administrator Centers for Medicare \& Medicaid Services Before the House Committee on Ways \& M eans Hearing on Medicare Prescription Drug Benefit: Department of Health \& Human Services USA; 2006. Available from: http://www.hhs.gov/asl/testify/t060503a.html.

Mello MM. Understanding medical malpractice insurance: A primer. The Synthesis Project, The Robert Wood Johnson Foundation, 2006 January 2006. Report No.: 8.

M ortality and Burden of Disease Estimates for WHO Member States in 2004. World Health Organization; 2009.

Naderi PS, M eier BD. Privatization within the Dutch context: a comparison of the health insurance systems of the Netherlands and the United States. Health (London). 2010;14(6):603-18.

Neonatal and Perinatal Mortality: Country, Regional and Global Estimates: World Health Organization; 2006.

OECD Health Data 2011 - Frequently Requested Data [Internet]. 2011 [cited 17.10.2011]. Available from: http://www.oecd.org/dataoecd/52/42/48304068.xls.

OECD. Health at a Glance Europe 2010: OECD Publishing; 2010. Available from: http://ec.europa.eu/health/reports/docs/health_glance_en.pdf.

OECD. How Does the United States Compare: OECD; 2011. Available from: http://health.citizing.org/library/ 1272.

Ogden CL, Carroll MD. Prevalence of Obesity Among Children and Adolescents: United States, Trends 1963-1965 Through 2007-2008. Health E-Stats [Internet]. June 2010. Available from: http://www.cdc.gov/nchs/data/hestat/obesity_child_09_10/obesity_child_09_10.pdf.

Ogden CL, Carroll MD. Prevalence of Overweight, Obesity, and Extreme Obesity Among Adults: United States, Trends 1960-1962 Through 2007-2008. Health E-Stats [Internet]. June 2010 17.10.2011. Available from: http://www.cdc.gov/nchs/data/ hestat/obesity_adult_07_08/obesity_adult_07_08.pdf.

Ottawa Charter for Health Promotion, First International Conference on Health Promotion, Ottawa, 21 November 1986: World Health Organization; 1986 November 17-21, 1986.

Prosser LA, Koplan JP, Neumann PJ, Weinstein MC. Barriers to using cost-effectiveness analysis in managed care decision making (Abstract). Am J Manag Care. 2000;6(2):173-9.

Q\&A: US healthcare reform at BBC News: BBC News; 2011 [24.06.2011]. Available from: http://newsvote.bbc.co.uk/mpapps/pagetools/print/news.bbc.co.uk/2/hi/americas/8160058.s tm?ad=1.

Reinhardt UE, Hussey PS, Anderson GF. U.S. Health Care Spending In An International Context. Health affairs. 2004;23(3):10-25.

Remarks by the President on Health Insurance Reform Glenside, Pennsylvania: The White House; 2010 [24.06.2011]. Press Release]. Available from: http://www.whitehouse.gov/the-pressoffice/remarks-president-senate-passage-health-insurance-reform.

Ricciardi W. EUPHA President's Column--EUPHA and WHO together in developing the new European policy for health. The European J ournal of Public Health. 2011;21(3):394. 
Rosenau PV, Lako CJ. An Experiment with Regulated Competition and Individual Mandates for Universal Health Care: The New Dutch Health Insurance System. J Health Polit Polic. 2008;33(6):1031-55.

Saltman RB, Bankauskaite V, Vrangbaek K. Decentralization in health care. Strategies and outcomes. Berkshire, England: Open University Press; 2007.

Saltman RB, Busse R, Figueras J. Social health insurance systems in western Europe. Berkshire, England: Open University Press; 2004.

Saltman RB, Figueras J, editors. European health care reform. Analysis of current strategies. Copenhagen: World Health Organization Regional Office for Europe; 1997.

Saltman RB, Figueras J. Analyzing the evidence on European health care reforms. Health Affairs. 1998;17(2):85-108.

Saltman RB. Health care reform. Int J Qual Health C. 1998;10(3):261-.

Savas BS, Cesuroglu T, editors. Sağlık Yöneticileri için Üreme Sağlığı Hizmetleri Yönetimi [Management of Reproductive Health Services for Health Managers]: Birlesik Saglik Sistemleri Ltd. (United Health Systems Ltd.); 2004.

Savas BS, Gedik G, Craig M. The reform process. In: M cKee M, Healy J, Falkingham J, editors. Health care in central Asia. European Observatory on Health Care Systems Series. Buckingham, Philadelphia: Open University Press; 2002. p. 79-91.

Savas BS, Karahan Ö, Saka RÖ. Health care systems in transition: Turkey. Thomson S, Mossialos E, editors. Copenhagen: European Observatory on Health Care Systems; 2002.

Savas BS, Ustunel A. Planning the implementation of Health Sector Reform in Central Asia. In: Feachem Z, Hensher M, Rose L, editors. Implementing health sector reform in Central Asia. Washington D.C.: The World Bank; 1998.

Savas BS. M ACH: a methodology for analysing contracting in health care. Copenhagen: World Health Organization, Regional Office for Europe; 2000.

Savas BS. Report on the implementation of health care reforms in Kyrgyzstan for the period May November 1997. World Health Organization, Europe \& Manas Health Care Reforms Programme of Kyrgyzstan, 1998.

Schäfer W, Kroneman M, Boerma W, Berg M vd, Westert G, Devillé W, et al. The Netherlands: Health system review2010.

Schoen C, Davis K, How SK, Schoenbaum SC. U.S. health system performance: a national scorecard. Health affairs. 2006;25(6):w457-75.

Shearer G. Prevention Provisions in the Affordable Care Act: American Public Health Association; 2010. Available from:

https://www.apha.org/ /media/files/pdf/topics/aca/prevention_aca_final.ashx.

Shortell SM, Gillies R, Wu F. United States Innovations in Healthcare Delivery. Public Health Reviews. 2010;32(1):190-212.

Silver MHW. Patients' rights in England and the United States of America: The Patient's Charter and the New Jersey patient bill of rights: A comparison. J Med Ethics. 1997;23(4):213-20.

Squires DA. The U.S. Health System in Perspective: A Comparison of Twelve Industrialized Nations: The Commonwealth Fund; July 2011. Available from: http:// www.commonwealthfund.org/publications/ issue-briefs/2011/jul/us-health-system-inperspective. 
Ståhl T, Wismar M, Ollila E, Lahtinen E, Leppo K. Health in All Policies: Prospects and potentials. Finnish Ministry of Social Affairs and Health, European Observatory on Health Systems and Policies, 2006.

Starfield B. Is US health really the best in the world? Jama-J Am M ed Assoc. 2000;284(4):483-+.

Stevens RA. History \& Health Policy in the United States: Putting the Past Back In - Investigator Awards in Health Policy. Research in Profile. 2007 September 2007.

Stevens RA. History and Health Policy in the United States: Putting the Past Back In. Apple R, Rosenberg CE, Burns LR, editors. New Brusnwick, New Jersey: Rutgers University Press; 2006.

Strandberg-Larsen M, Nielsen MB, Vallgårda S, Krasnik A, Vrangbæk K. Denmark: Health system review. Copenhagen: World Health Organization on behalf of the European Observatory on Health Systems and Policies; 2007.

Tanne JH. American Medical Association backs health reform despite challenges from some members. Brit M ed J. 2011;342.

Tanne JH. Obama administration wins first appeal court ruling on health reform. Brit Med J. 2011;343.

Taylor CE. Learning from Health-Care Experiences in Developing-Countries. Am J Public Health. 1993;83(11):1531-2.

The Burden of Chronic Diseases and Their Risk Factors: National and State Perspectives: U. S. Department Of Health and Human Services, Centers for Disease Control and Prevention; 2004. Available from: http://9healthfair.publichealthpractice.org/documents/burden_book2004.pdf.

The Commonwealth Fund Commission on a High Performance Health System. Why Not the Best? Results from the National Scorecard on U.S. Health System Performance: The Commonwealth Fund; 2011. Available from: http://www.commonwealthfund.org/publications/fundreports/2011/oct/why-not-the-best-2011.

The Economic Case for Health Care Reform: Executive Office of the President, Council of Economic Advisors; $2009 . \quad$ Available from: https://www.whitehouse.gov/administration/eop/cea/TheEconomicCaseforHealthCareReform l.

The Future of the Public's Health in the 21st Century. Washington D.C.: The National Academies Press; 2003. Available from: http:// www.nap.edu/openbook.php? record_id=10548.

The Ljubljana Charter on Reforming Health Care in Europe: World Health Organization Regional Office for Europe; 1996 18.06.1996.

The Staff of the Washington Post. Landmark: The Inside Story of America's New Health Care Law and What It M eans for Us All: PublicAffairs; 2010.

The Vision, M ission, and Goals of Healthy People 2020: U.S. Department of Health and Human Services; 2010. Available from: http://healthypeople.gov/2020/about/default.aspx.

Transformation in Health: M inistry of Health, Republic of Turkey; 2003 December 2003.

Underinsured in America: Is Health Coverage Adequate? [Fact Sheet]. Washington D.C.: The Henry J. Kaiser Family $2002 . \quad$ Foundation; Available from: http://www.kff.org/uninsured/loader.cfm?url=/commonspot/security/getfile.cfm\&PagelD=14 136.

Update on Funding and Implementation of Public Health and Prevention Provision in the Patient Protection and Affordable Care Act (ACA). American Public Health Association, Center for Public Health Policy, 2011 June 2011. Report No. 
van de Ven WPM M, Schut FT. Universal mandatory health insurance in the Netherlands: A model for the United States? Health affairs. 2008;27(3):771-81.

Vienonen M, Jankauskiene D, Vask A. Towards evidence-based health care reform. B World Health Organ. 1999;77(1):44-7.

Vuorenkoski L. Finland: Health System Review. Mladovsky P, Mossialos E, editors. Copenhagen: World Health Organization on behalf of the European Observatory on Health Systems and Policies; 2008.

Westert GP, van den Berg MJ, Zwakhals SLN, Heijink R, de Jong JD, Verkleij H. Dutch Health Care Performance Report 2010, Executive summary. Bilthoven: National Institute for Public Health and the Environment (RiVM); 2010. Available from: http:// www.gezondheidszorgbalans.nl/dsresource? type=pdf\&disposition=inline\&objectid=riv $m p: 256249 \&$ versionid $=\&$ subobjectname $=$

Wiegers TA, Hukkelhoven CW. The role of hospital midwives in the Netherlands. BMC Pregnancy Childbirth. 2010;10:80.

Wismar M, M cKee M, Ernst K, Srivastava D, Busse R. Health targets in Europe. Learning from experience. Copenhagen: World Health Organization on behalf of the European Observatory on Health Systems and Policies; 2008.

Woolhandler S, Campbell T, Himmelstein DU. Costs of health care administration in the United States and Canada. New Engl J M ed. 2003;349(8):768-75.

Yong PL, Saunders RS, Olsen L. The Healthcare Imperative: Lowering Costs and Improving Outcomes: Workshop Series Summary. Washington D.C.: The National Academies Press; 2010. Available from: https://www.iom.edu/Reports/2011/The-Healthcare-Imperative-Lowering-Costs-andImproving-Outcomes.aspx.

Zwar NA. Health care reform in the United States: an opportunity for primary care? M ed J Australia. 2010;192(1):8-. 
PART II

WHAT TO INTEGRATE? 
The worst form of inequality is to try to make unequal things equal. Aristotle 


\section{Chapter 4}

\section{Debating the definition of personalized approaches: Content analysis of practices}

Tomris Cesuroglu, Elena Syurina, Frans Feron and Anja Krumeich

The Other Side of the Coin for Personalized Medicine and Health Care: Content Analysis of 'Personalized' Practices in the Literature. Submitted. 


\begin{abstract}
Background

Various terms and definitions are used to describe personalized approaches to medicine and health care, but in ambiguous and inconsistent ways. They mostly have been defined in a top-down manner. However, actual practices might take different paths. Here, we aimed to provide a 'practice-based' perspective on the debate by analyzing the content of 'personalized' practices published in the literature.
\end{abstract}

\title{
Methods
}

The search in PubMed and EMBASE (April 2014) using the terms frequently utilized for personalized approaches resulted in 5.333 records. Two independent researchers used different strategies for screening, resulting in 157 articles describing 88 'personalized' practices that were implemented or presented on at least one individual/patient case. The content analysis was grounded on this data and didn't have a priori analytical frameworks.

\section{Results}

'Personalized medicine and health care' can be a commodity in health care market, a way how health services are provided, or a keyword for emerging applications. It can help individuals/patients to gain control of their health, health professionals to provide better services, health care organizations to increase effectiveness and efficiency, or national health systems to increase performance. Country examples indicated that for integration of practices into health services, attitude towards innovations and health system and policy context is important. Categorization based on the terms or the technologies used, if any, wasn't possible.

\section{Conclusions}

This study is the first to provide a comprehensive content analysis of the 'personalized' practices in the literature. Unlike the top-down definitions, our findings highlighted not the technologies but real life issues faced by the practices. 'Personalized medicine' and 'personalized health care' can be differentiated by using former for specific tools available and latter for health services with a holistic approach, implemented in certain contexts. To realize integration of personalized medicine and health care into real life, science, technology, health policy and practice, and society domains must work together. 


\section{Introduction}

A trend towards 'personalization' in medicine and health care has become increasingly prominent in the past 10-15 years. It has been perceived and described from different angles by various opinion leaders, institutions and stakeholder organizations. The terms used include 'personalized medicine', 'personalized health care (healthcare)', 'P4 Medicine - Predictive, Personalized, Preventive/ Preventive, and Participatory Medicine', 'individualized medicine', 'precision medicine' and 'systems (bio)medicine'.

These terms are heavily used in both scientific and grey literature. However, until now, there has been no consensus on their precise meaning and how they are distinguished. The situation is complicated by ambiguous and inconsistent use of terminology. For example, in some cases two terms are used interchangeably (e.g. individualized medicine and personalized medicine at Medical Subject Headings of PubM ed [1]) whereas in another, each are used to describe different concepts [2]. Each individual, research group or manuscript seems to have a preferred term and working definition for it. Moreover, many publications seem to use 'personalized medicine' as a buzz word and/or a 'catch-all' term, resulting in a steep increase in its appearance in the literature $[3,4]$. These create chaos within terminology in the literature. Within the present article, the abbreviation PM/PHC will be used to address the wide scope of the personalized medicine, personalized health care and others.

Problems with the terminology have been a subject to several publications [2-10], the most recent of them being a comprehensive overview by Pokorska-Bocci [3]. So far, influential definitions and descriptions of the field have mainly been launched by institutions [11-14], organizations representing major stakeholder groups such as industry and academia [15-17], and respected opinion leaders [18-23]. The body of literature that addressed or discussed the terminology has mostly referred to these influential 'top-down' definitions. There is an implicit assumption that there is such thing as a best definition or classification of PM/PHC and that the practices in this field will intend to develop accordingly. However, as the studies in other advanced technological domains have shown [24], in reality, actual practices in health care might take their own path, thus 'enacting' in their practices implicit definitions of what is at stake in their field. These definitions then determine what direction the field is heading towards.

The problem with the definitions and terminology is not just a linguistic one. In the broader context, it is very likely to be connected with the bigger problem of unexpectedly slow integration of personalized practices in real life. PM/PHC has been on the agenda of scientific fora for over a decade. Personalized approaches were proposed to be a solution to the main problems of today's health systems, including prevention and treatment of chronic complex diseases, which are responsible for the main burden of disease globally $[19,23,25,26]$. However, PM/PHC hasn't fulfilled the expectations so far. Diffusion of 
practices in regular health services hasn't taken place with the exception of cancer related programs in some countries. Can the underlying assumptions and origins of the top-down definitions be contributing to this problem? If so, can the practice side of the coin give us clues on what matters for integration of personalized approaches in health care?

Inspired by these ideas, we decided to look at the PM/PHC practices that are emerging in the literature, which use various terms listed above. We assumed that these practices actually reflect their implicit views on PM/PHC and their analysis may provide better insight in what is happening on the practice side of the field. Thus, this study aims to start developing an understanding of how the field can be defined from a 'practice-based' perspective. This can make a valuable contribution to the debate on the terms and definitions, and help seeing how the field is actually evolving. We will then look at what the findings mean for integration of PM/PHC into real life. In the discussion, we will compare the implicit notions on PM/PHC as enacted in actual practices with notions underlying the 'top-down' definitions, and propose a 'practice-based' look the health care integration problem in the field and possible solutions.

\section{Methods}

\subsection{Approach}

In order to approach the field from a 'bottom-up' or 'practice-based' perspective and avoid the influence of the top-down definitions, we needed to be careful about common assumptions seen in 'top-down' definitions. We identified two of them as important.

First one is linked to the drivers of the personalized vision. Two drivers seem to have a prominent place in the background of top-down definitions: 1) the general societal trend towards personalization and 2) the scientific discoveries and technological developments in various fields, mainly genome-based and information/communication technologies (ICT) [2]. The top-down definitions seem to be influenced in particular by the second driver. M ost of them tend to define the field from the perspective of a certain sector or discipline, such as by the use of certain interventions (drugs, etc.) or technologies (genomics, ICT, etc.) $[1,11,12,15,18,19]$. In this work, we took a sector, discipline- and technology- independent stand, which can help us identify new innovative approaches that are emerging using tools and disciplines that are not traditionally covered in descriptions of the personalized approaches.

Second assumption we avoided was thinking in silos of the 'pipeline' approach to innovations in health care. In the top-down approaches, innovative practices or interventions are often perceived to be an end result of the research and (technological) development process. The whole process is seen as stages, such as basic research, applied research, development, pilot and implementation, which are assumed to follow one another in a linear way. However, in real life, neither scientific advancements nor technologies get into 
health practice in a predictable pattern or 'pipeline' [27]. Innovations stemming from actual practices might not necessarily follow those in a structured way and the borders between these stages are not always clear [24].

\subsection{Systematic search and selection strategy}

In order to identify the practices, we used a systematic search and selection strategy summarized in Figure 1. It is based on the PRISM A flow diagram [28], modified according to the purpose of our study. For full details of the process, refer to Supplementary material 1.

\section{Information sources and search strategy}

PubMed (via PubMed.gov on 1 April 2014) and EMBASE (via OVID on 14 April 2014) were chosen for the search as they cover the highest number of journals related to 'personalized medicine'.

The following terms were searched in the title or abstract of the manuscripts: personalized health care (healthcare), personalized medicine, P4 medicine, precision medicine, stratified medicine, systems (bio)medicine, individualized medicine and their UK English versions (personalised and individualised). The search was limited to English articles, with no time limitations.

The search within PubMed resulted in 4.317 records and 6.328 in EMBASE, all of which were imported to EndNote. Duplicates and meeting/conference abstracts were removed. This resulted in 5.333 records.

The search was restricted to scientific publications only and did not include websites, reports and other grey literature sources, since it not always possible to assess the validity of claims made about the practices in those mediums.

\section{Screening and selection process}

The focus of this study is practices presented in relation to PM/PHC. Since 'health practice' or 'medical practice' doesn't have a formal and/or operational definition, we used the concept of 'health intervention', as "...an activity performed for, with or on behalf of a person or a population whose purpose is to improve, assess or modify health, functioning or health conditions." [29]. Thus, our inclusion criteria focused on articles that describe a health intervention that is developed (designed) and/or implemented and presented in association with one of the terms used to describe PM/PHC (see the search terms). 


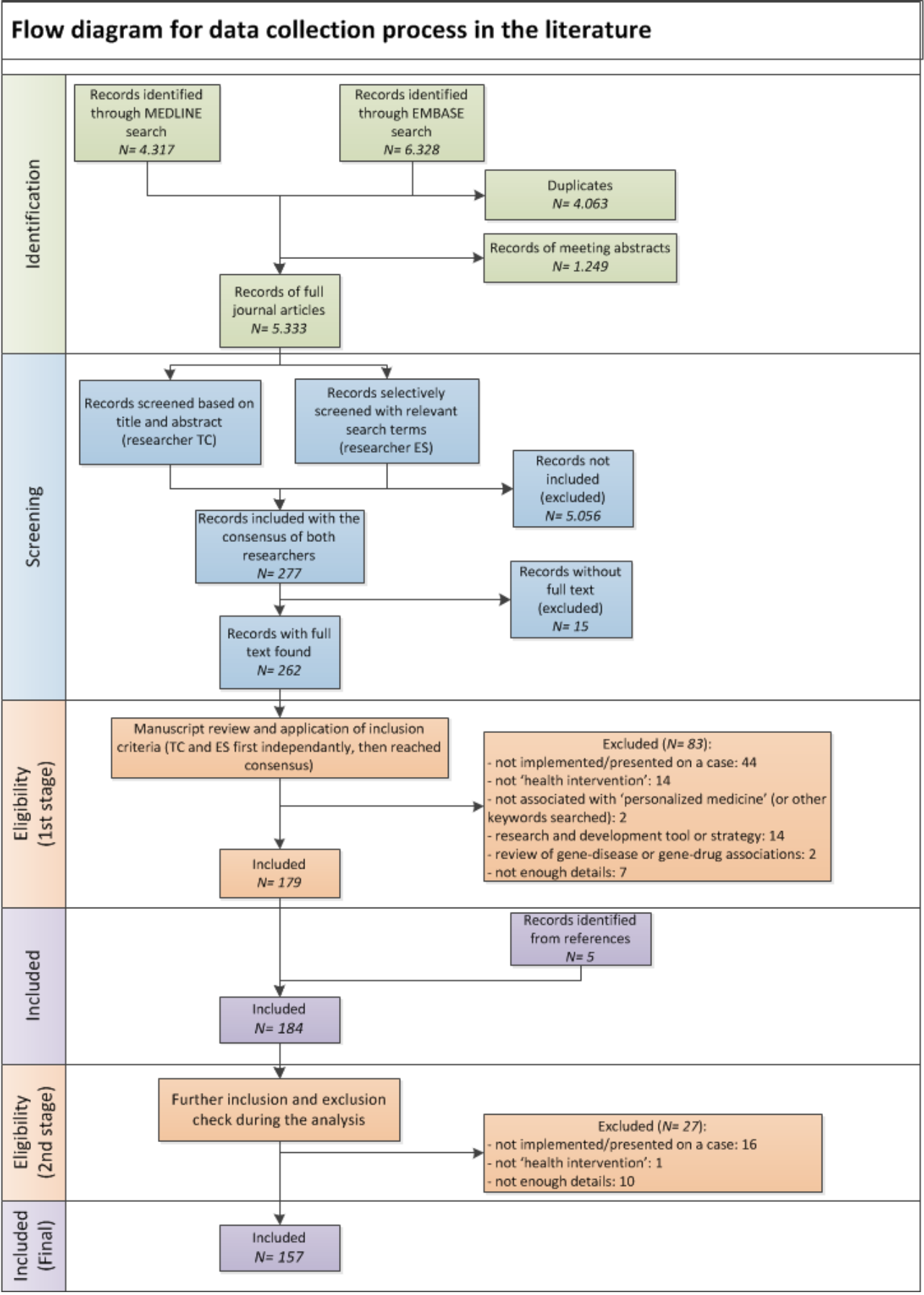

Figure 1: Flow diagram of data collection process 
More precisely, we searched for health intervention/ practices that 1) were implemented on at least one real-life case or had contacted with the patient/ individual or his data (i.e. a case, series of cases, feasibility studies, pilots, trials, reports on products/ services on the market; etc.) and 2) had enough description details provided in the article(s). The stage of research, development and implementation, how well the implementation is established, or the technological components were not included in the analysis.

The selection was done independently by two researchers (TC and ES) on two occasions. First, during the initial screening, resulting in 277 articles and second, during the eligibility screening. Within the screening phase, independent researchers used two different strategies for triangulation. The results of both researchers were compared and differences were discussed until consensus was reached. At this stage 179 articles were included. Upon inclusion some of the articles identified from the references and exclusion of those not providing enough information, the end list of 157 articles was formed. The reasons for exclusion are summarized in Figure 1.

\subsection{Identification of practices from the articles}

Practices were extracted from the articles, different articles describing the same practice were identified, and list of the practices was formed. From this stage onwards, practices (not the articles) became the unit of analysis in this study. Some practices could be clustered under certain groups, based on their similarities. For example, various drug-genetic test pairs could be grouped under 'pharmacogenetics', since no apparent differences were expected during the analysis and the available data in the articles was also presented to support grouping. Eventually, 9 groups of practices were formed (treated as one practice in the rest of this article) in addition to 79 individual ones, summing up to 88 practices (see Table S1 in Supplementary material 2). The "P- numbers" in Table S1 are used as the identifier of each practice in the rest of the article and referred with a "P-" in front of it in brackets. For example, (P-1) refers to "Group: Pharmacogenetics (drug - gene)". Details of the group of practices are presented in Table S2 in Supplementary material 2.

\subsection{Content analysis}

The author (TC) read the articles, investigated the practices from different perspectives, identified their distinctive properties and discussed various categorization possibilities with the co-authors (ES, AK, FF). We decided to start with the axis of 'application form and context', since many articles provided ample information on that topic (Axis-1). While comparing similarities and contrasting differences among the practices within and across categories, the categorization was refined and re-defined. This resulted in reorganization of the practices under the categories. This took several rounds, until the description of the categories matched with the content of the practices included and the categories became mutually exclusive. 
The second area we decided to investigate focused on "who is served by this practice?". This involves 'users' or 'clients' (Axis-2). Using a method similar to Axis-1, we identified various groups who are eventually served by this practice and clustered them in 4 groups. Many of the practices served to more than one group, some of which were minor or optional clients, or implied to be served in the articles. We preferred to note these in the tables as well (with notes such as 'minor', 'optional', 'indirect') because many of the practices are in the conception or infancy phases now and in the future may evolve to directions that seem minor now. Eventually, the groups that emerged in this axis were not mutually exclusive. Therefore, we called them 'groups' rather than 'categories'.

During several discussions we identified that issues such as who the practice is available for, who controls access to it and who benefits from it are important topics for integration of practices into (health) services. However, these topics are tightly related to the health system, including the health financing, of the country where the practice is implemented. However, as we collected our data from the scientific literature, where information on revenue models, benefits, availability, access etc. are not reported regularly, we chose to present three country examples to compare how the practices are integrated.

The analysis was made by the author, and co-authors challenged it providing critical feedback in several rounds. The discussions among the team continued until an agreement on the axes and their components was reached. MSExcel was used for the analysis.

Before and after the analysis process summarized above, we also explored if there is an identifiable pattern in the use of different terms referring to the personalized approaches. However, we didn't observe a consistent pattern in their use in terms of technologies and/or disciplines involved, the purpose (prevention, therapy, disease management, etc.), target groups, etc. We didn't observe a pattern in terminology according to the categories we identified either.

After the analysis, the results were summarized into tables, where short descriptions based on the articles were added. Since we based the analysis on only the articles, changes that happened afterwards the publications, such as stopping service or changing its form, were not taken into account. For practices that were reported to have a web site, the web sites were checked to see if the provider has apparently changed or stopped services after the publication and this was put as a brief note in the relevant tables as necessary.

\section{Results}

The basic properties of the 88 practices identified from the articles are presented in Table $\mathrm{S} 1$ in Supplementary material 2. Figure 2 presents the general view of the axes and their components, which are summarized in below text with examples from the practices. 


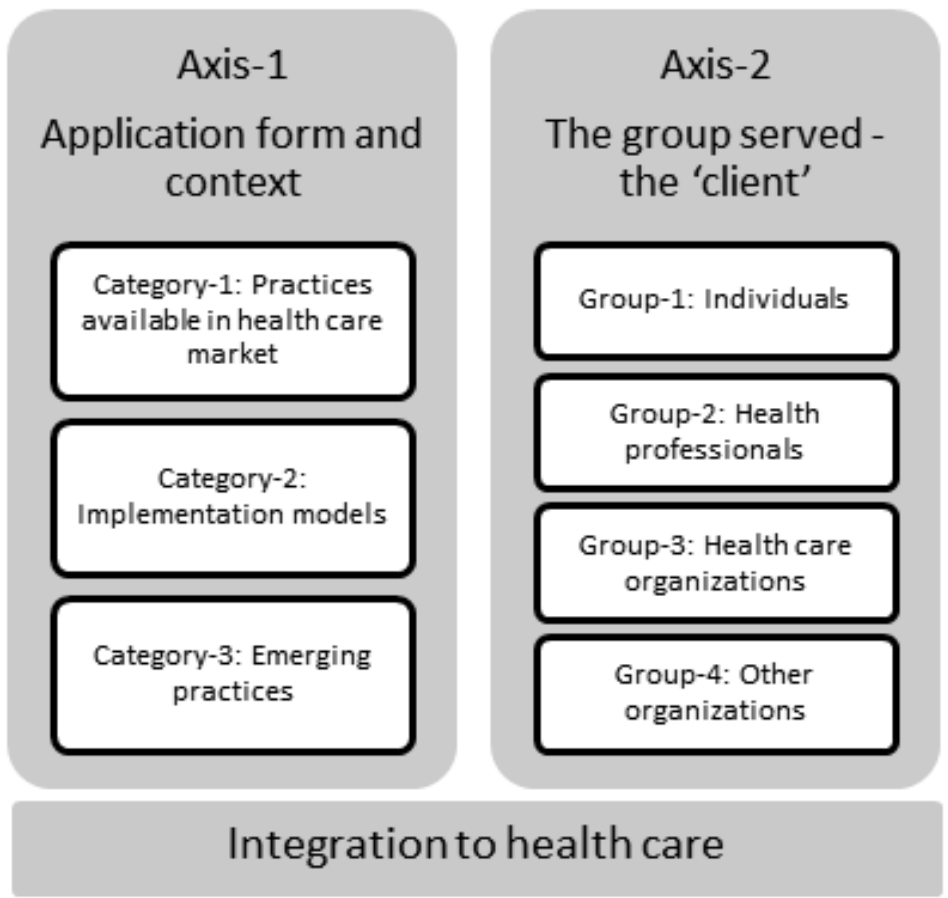

Figure 2: The axes and their components that emerged from the analyzed practices

\subsection{Axis-1: Application form and context}

Data analysis revealed three main categories in terms of the application form and context: 1) Practices available to be used in health services, implying that PM/PHC is a commodity that is provided on the health care market; 2 ) Implementation models where the product or service is developed and/or implemented in a wider context (i.e. in an institution), implying that PM/PHC is a way how health services are organized and provided; and 3) Emerging practices which haven't evolved to one of the first two groups yet, implying $\mathrm{PM} / \mathrm{PHC}$ as keyword which indicate a 'future' or 'novel' potential.

All practices were categorized in only one of the three categories, except for Gentest (P20) [26] and Genelnsight (P-32) [30] as these two practices had two service facets. Practices are summarized in separate tables for each category in Supplementary material 2 (Tables S3, S4 and S5 for Category-1, 2 and 3, respectively).

\subsubsection{Category-1: Practices available in health care market}

This category includes products/services that are available in the health care sector/market. Their uptake and use depends on the decision of the health professional and/or individuals/patients. Tests, test-drug pairs, drugs, (bio)informatics services, devices, online tools, and web sites fall into this group. Most of these are commodities available 
on health care market, which have a cost for the payer (whomever the payer is, i.e. third party payer, the patient or others) just as other services and products in health care. An example is pharmacogenetic tests and related drugs (P-1), which are ordered by the physician. (see Box 1)

A few interventions are available publicly, not commercially, e.g. the tools available freely in the literature and internet for predicting the risk or prognosis of a disease, such as coronary risk scores (P-12). (see Box 1 )

\section{Box 1: Examples to practices available in health care market (category-1)}

\section{Pharmacogenetic tests and related drugs (P-1)}

In pharmacogenetic testing, the sample of the patient is tested for a certain (genetic or genomic) biomarker in the laboratory to predict the response of the individual to a certain drug. The physician can use this information to choose the most appropriate drug or decide its dose. For example, individuals respond differently to warfarin, an anticoagulant drug used for prevention and treatment of thrombosis and thromboembolism. Therefore the warfarin dose must be titrated according to the response of the individuals, as measured by the INR (International Normalized Ratio) value in the blood. Therapeutic index for INR is rather narrow, and higher and lower than desired levels increase risk of serious complications (hemorrhage) and lack of desired effect (thrombosis). Genetic polymorphisms in CYP2C9 and VKORC1 genes are responsible for the $40-60 \%$ of the variability in the therapeutic warfarin dose. Testing individuals for these polymorphisms before the initiation of warfarin therapy can help to optimize the starting dose of the warfarin and might reduce the risk of hemorrhagic complications [31-36].

\section{Coronary risk scores (P-12)}

The risk of cardiovascular diseases can be calculated using classical scoring systems such as Framingham risk score (USA) [37]. The scoring is available in the literature or can be calculated automatically in the internet when the personal clinical variables of the individuals, such as age, gender, total and HDL cholesterol levels, smoking status and hypertension/ blood pressure information are entered (http://cvdrisk.nhlbi.nih.gov/).

\subsubsection{Category-2: Implementation models}

This category includes products or services that are developed for and/or implemented in a certain context; i.e. a health care institution, a country and worldwide. Practices can be run under a project, a program or a service framework that aims to implement, pilot, or assess feasibility of the implementation of products or services, as well as related tools in a certain setting. The implemented products or services can already be available on the market (category-1) or developed specifically for that setting. These PM/PHC practices imply that PM/PHC is not just a tool, product or service available (category-1), but also the way how health services are organized and delivered. 
We labeled the practices in this category broadly as 'implementation models' because they not only focus on the product/ service or its technical use, but also take into account its implementation within an organization or setting. By definition, they all are expected to involve a health professional for their administration, except Athlete Biological Passport (explained below). Examples to three implementation contexts are presented below. (see Box 2)

1. Institutional context: Projects, programs or service frameworks developed for and/or implemented at a specific health care institution are included under the institutional context'. They can be as small as a physician practice, or as large as a chain or group of major academic medical centers, including home health services.

A variety of practices are implemented in an institutional context. For example, a number of programs explore, develop and pilot the ways of implementing pharmacogenomics in clinical practice in major medical centers (P-25, P-57, P-62, P-66, P-68, P-82). Some of the practices are service frameworks that are developed at specific institutions, focusing on other aspects of personalizing the health services, such as psychosocial needs (P-86).

2. National context: The only example of implementation in national scale is the French National Cancer Institute's (French $\mathrm{NCl}$ ) nationwide program for tumor genomics (P-44).

3. International context: Currently, the only practice in this subcategory is Athlete Biological Passport (P-42), a program offered by World Anti-Doping Agency and implemented by anti-doping agencies around the globe.

\section{Box 2: Examples to implementation models (category-2)}

\section{Pharmacogenetics related programs (P-25, P-57, P-62, P-66, P-68, P-82)}

A number of programs involve genotyping a group of patients for identified genotypes before the relevant drug and testing is indicated (preemption), and aim to investigate the results of this practice in various aspects, such as, outputs, patient outcomes and costs, and others: CLIPMERGE PGx Program at Mount Sinai Medical Center (P-25) [38], PREDICT project at Vanderbilt (P-57) [39], Personalized Medicine Program at University of Florida Health (P-62) [40], Clinical Pharmacogenomics Implementation Program at University of Chicago (P-66) [41, 42], Coriell Personalized Medicine Collaborative (Pharmacogenomics) (P-68) $[43,44]$, and Preemptive PGx protocol at M ayo Clinic (P-82) [45], all in USA.

\section{Youth Area for adolescent and young adult cancer patients (P-86)}

'Youth Area' at CRO National Cancer Institute, Italy is a special unit for adolescents and young adults at a cancer research hospital to provide assistance to them, in particular for their psychosocial needs [46]. 


\section{French National Cancer Institute's (NCI) nationwide program for tumor genomics} (P-44)

The program aims to provide available genomic tests to all relevant cancer patients in France. French $\mathrm{NCl}$ runs it with a network of 28 laboratories in France. Each laboratory is located in a specific geographic area to provide tumor genomics services to all cancer (public and private), thus covering whole France. Funded by the French $\mathrm{NCl}$ and Ministry of Health, all patients have free access to the identified biomarkers that predict response to certain cancer treatments [47].

\section{Athlete Biological Passport (P-42)}

Athletes' are monitored longitudinally with tests on hematologic and steroidal profiles. Recent changes and disparities in the parameters can be a result of performanceenhancing drugs or a medical condition that needs to be investigated. The program is developed by the World Anti-Doping Agency. Anti-doping agencies around the globe can implement it using the relevant guidelines [48].

\subsubsection{Category-3: Emerging practices}

Practices in this category include remaining ones. They have made a 'contact with the patient (data)', however information on its current use is not (yet) available in the literature. These, unlike practices in category-1, are not yet available on the market (at the time of the data collection) and are not developed for a specific (institutional) context like the ones in category-2. They may evolve into these two, or to other directions. (For examples, see Box 3)

We didn't observe a pattern by which the practices per se can be grouped, but the ways they are reported in the articles seem to have three prominent ways:

1. Demonstration of applicability or proof of concept: These practices were applied or presented on cases (one or more) or datasets, such as the 'IT-based Diagnostic Instrument System' (P-13), a personal wearable device and home tele-health system for measurement of a variety of parameters and bio-signals of the individuals. The applicability of algorithms or bioinformatics tools are demonstrated by applying them on datasets, such as the algorithm that predict the outcome of gliomas based on their transcriptomic profile, GliomaPredict (P-28).

2. Trial implementation: These practices are implemented specifically for research purposes in one or more settings. This can be a pilot to assess feasibility, usability, clinical validity and utility (efficacy and effectiveness). Unlike the pilots reported in category-2 of Axis-1, which were programs developed for specific institutions, here, the product or service is not developed for the setting where it is tested. 
An example is the Computer-Assisted Brief Intervention for Tobacco (CABIT) Program (P47), which is a web-based, multimedia tobacco intervention for use in opportunistic settings. It was piloted in a number of health care settings for its initial evaluation including aspects of patient and provider satisfaction and treatment initiation. It is unknown at this point if it became a long-term program for those settings or if it became available for different settings. Another example explored physicians' reactions to the prototype of an algorithm and reporting system for a pharmacogenetic test (CYP3A5 - tacrolimus dose) (P85).

3. Exploratory/investigational cases: These cases initially had an investigational or exploratory purpose, without knowing if it would have specific health practice implications. $A$ specific health practice wasn't (fully) designed prior to the start of the case, but eventually led to a health practice. 'integrative Personal Omics Profile (iPOP)' project (P-50) carried out longitudinal extensive omics profiling on blood components from a generally healthy individual. Another individual followed various parameters related to his body longitudinally using digital biomedical measurement devices and blood and stool biomarkers ( $P$ 59). In both examples, the follow-up led to clinical diagnosis of diseases.

\section{Box 3: Examples to emerging practices (category-3)}

\section{IT-based Diagnostic Instrument System (P-13)}

It is a set of diagnostic medical instruments which have been integrated into a personal wearable device (wrist-worn health monitoring device), which measure a variety of parameters in the body, a blood glucometer integrated to a cell phone and a home telehealth system including bio-signal measurement systems that can measure, for example, ECG, body temperature, body-fat ratio on a toilet seat. It is developed at Seul National University (Korea) [49].

\section{GliomaPredict (P-28)}

GliomaPredict is an algorithm for prediction of the outcome of gliomas (a brain tumor) based on their transcriptomic profile. It is validated on datasets and available for use in one institution, National Cancer Institute, USA [50].

\section{Computer-Assisted Brief Intervention for Tobacco (CABIT) Program (P-47)}

CABIT is an opportunistic computer based tool to assist quitting smoking in clinical settings (Cooper University Hospital \& Polaris Health Directions, Inc., USA). It is a self-administered computerized assessment to produce reports for health care provider and patient. Based on the assessment, the tool provides a video intervention to the individual (based on the stage-of-change for the individuals smoking habit) and provides referral for smoking cessation [51]. 


\section{A prototype for automated interpretation of a pharmacogenetic (CYP3A5 - tacro- limus dose) (P-85)}

Tacrolimus is a drug used to prevent rejection in organ transplantation. The needed dose for this drug may change according to the CYP3A5 genotype. An algorithm and reporting system was developed for CYP3A5 test and recommending tacrolimus dose at Oslo University Hospital, Norway [52].

\section{integrative Personal Omics Profile (iPOP' project) $(P-50)$}

Extensive omics profiling of blood components from a generally healthy individual was performed over a 14 month period at Stanford University, USA. The whole genome sequencing data was analyzed with RiskOGram algorithm, which integrates information from multiple alleles associated with disease risk. It indicated an increased risk of type 2 diabetes, which actually developed during the follow-up period and was diagnosed by a physician [53]. The primary aim of the project was not to provide a 'health practice'. However, the analysis made on the whole genome data, i.e. risk assessment of various diseases, had implications as a health practice.

\section{Quantified-self (P-59)}

An individual followed various parameters related to his body longitudinally using digital biomedical measurement devices and blood and stool biomarkers (USA). He observed that episodically elevated stool protein markers were accompanied by increased blood CRP levels, indicting inflammation. He got colon imaging studies and biopsies, which diagnosed that he had a late-onset Crohn's disease, which is an inflammatory bowel disease [54].

\subsection{Axis-2: The group served by the practice, i.e. the 'user/client'}

This axis involves with the question "who is the main group or entity served by this practice?" In other words, "who turns out to be the main 'user' or 'client' of this practice, and what definition of PM/PHC does this imply"?

Data analysis revealed 4 main groups: 1 ) Individual (including the patient), implying that (PM/PHC) means tools that involve and/or empower the individuals to gain control over matters related to their health; 2) Health professionals, implying that PM/PHC tools help them provide better tailored and therefor better quality health services; 3 ) Health care organizations, implying that PM/PHC means ways to increase effectiveness and efficiency of health care delivery; 4) Other organizations, such as a payer organization, a national health system and international system.

Axis-2 is summarized in Tables S3, S4 and S5 as a separate column in Supplementary material 2. 


\subsubsection{Group-1: Individuals}

If the articles indicate that the practice is used by the individual for management of their health or disease (risk), it is considered to serve the individual. The term individual covers both 'patients', i.e. people with disease, and healthy individuals. (For examples, see Box 4)

A prominent example here is direct-to-consumer genetic tests that identify predisposition to or predict risk of certain diseases (P-6). The individual can order the test and receive the results without the involvement of a health professional. Another example includes various smartphone based personal, pervasive health informatics services that support individuals' self-care to stay healthy and prevent diseases (P-9).

The practices serving primarily to the individual place him/her in the center of the service and help them be more involved with their health. Here, personalization implies empowering persons to take care of themselves or to gain control of their own health.

This group also includes practices that serve the people surrounding the individuals, such as families of the adolescents and young adults in cancer wards (P-86) (see Box 2). This is considered as a continuum of the 'individual centered' approach; taking the individual with people who can support or help them.

\section{Box 4: Examples to practices serving to individuals (group-1)}

\section{Direct-to-consumer genomic tests ('personal' or 'consumer' genomics) (P-6)}

These tests are also called 'consumer genomics' or 'personal genomics' services, commonly exemplified by 23andMe [55-59]. 23andM e, a U.S. company, provides genome wide DNA test ("Personal Genome Scan") over the internet directly to consumers. A saliva sample of the individual is used to detect variations in the DNA, with the purpose to provide information on two areas: ancestry/genealogy and health. Under the 'health' section, disease risks based on common polymorphisms as well as highly penetrant variations, carrier status of monogenic disorders, some physical traits and response to drugs are provided online as results of the test. Individuals are free to take these to their doctors for discussion or medical follow-up. (This description is based on the articles included, with the latest from 2012. It is of note that the content of 23andM e changed in December 2013 due to the warning of Food and Drug Administration of USA [60] and the company no longer offers the 'health' section to new customers [61]).

\section{Smartphone-Based Personal, Pervasive Health Informatics Services (P-9)}

Smartphone-based personal health informatics, including sensor, signal and imaging informatics are applications for provision and improvement of diagnosis, acute and chronic treatment, and rehabilitative health services as well as supporting self-care to stay healthy/prevention of diseases [62]. Many of these services are available for the individu- 
als, for example, for fitness monitoring using GPS and accelerometers, sensors for heart rate monitors, and devices that combine these to a wrist-worn device such as FitBit.

\subsubsection{Group-2: Health professionals}

If the health professionals use the practice for medical management of the disease (risk) or the patient, it is considered to serve this group. Laboratory tests ordered by the physician and the drugs prescribed are included here because they mainly assist the medical management of the disease, carried out by the physician and other health professionals $A$ prominent example here is tumor genetics/genomics tests and related drugs (P-2). (For examples, see Box 5)

An electronic questionnaire that identifies the psycho-cognitive status of the patients before the physician meets them (ALGA-C, P-84) provides feedback to the physician to optimize his/her communication style and interaction with the patient. Thus, the questionnaire is filled in by the patient but it eventually is a tool that serves the physician.

A few practices serve primarily both the individuals and the health professionals. One of them is Gentest, which is a preventive practice model provided to individuals via authorized physicians (P-20). The output of the service is a report, which includes, among others, the summary of assessments made for the individual, an optimum lifestyle program and a medical follow-up program, thus serving to both the individual and the physician.

The practices that primarily serve the health professional are PM/PHC tools that help the health professional (mostly a physician) deliver even better or more accurate health services to the individuals, potentially leading to more successful prevention, diagnosis and treatment. They can be perceived as 'high-profile' or elaborate products or services used by professionals.

\section{Box 5: Examples to practices serving to health professionals (group-2)}

\section{Tumor genetics/ genomics tests and related drugs (P-2)}

Here, the laboratory test is carried out on the tumor tissue, to predict its response to a given cancer drug [63-68]. The technology used for the test varies: genetic tests (specific mutation or sequencing), gene expression analysis, or immunohistochemistry, in situ hybridization, etc. In the recent years, there have been increasing number of cases of pairs of cancer drugs and accompanying laboratory tests (companion diagnostics), which are developed, received regulatory approval and presented to the health care market together $[33,66,67,69-73]$.

ALGA-C (questionnaire that measures and collects psycho-cognitive information about patients) (P-84)

ALGA-C is an electronic questionnaire focusing on cognitive, physical-related and psychological aspects of the patients. It is filled by the patients before meeting with the doctor. 
The results provide a psycho-cognitive picture of the patient with whom the physician is about to meet. The physician can use this information to optimize the communication style and interaction with the patient. It is developed by the P-medicine project (Greece, Italy, UK) [74].

\section{Gentest (P-20)}

Gentlest is an integrative preventive model that utilizes an individual's personal health information, family history, detailed lifestyle analysis (nutritional habits, food consumption using a food portion size atlas, physical activity and exercise, and smoking), anthropometric and bioimpedance measurements, genotype, and other biomarkers to stratify individuals into risk groups to prevent complex diseases in a targeted way. It is provided to individuals via authorized physicians. Based on the results of the aforementioned components, risk assessments are made for the major complex diseases including heart attack, stroke, type 2 diabetes, cancers (lung, breast, prostate, colon, and gastric cancers), and osteoporosis and an optimum lifestyle plan, including personal menu plans and food exchange lists, exercise plans, smoking cessation recommendations, and a medical follow-up plan are drawn. It is developed by GENAR Institute for Public Health and Genomics Research in Turkey [26].

\subsubsection{Group-3: Health care organizations}

Practices which have a major organizational component within the health services, such as involvement of different stakeholders (disciplines/departments) within an organization or across organizations for development and implementation of it, clinical and/or laboratory workflows, and targeting to increase effectiveness and efficiency in a health care institution, are included here. These are actually issues that are related to the goals of a health care provider institution, i.e. effectiveness, efficiency (costs) and patient and employee satisfaction. Thus, PM/PHC becomes tools or ways for health care organizations to perform better in their services, which may help to improve their businesses in the competitive environment. (For examples, see Box 6)

The Mayo Clinic's Individualized Medicine Clinic (P-71) is an example of an initiative that opened a new service line within an organization. This initiative involved several issues such as setting up and/organizing the infrastructure (laboratory, coordination, systems to handle samples and report results, education materials, etc.), putting the clinical workflow, and putting the administrative workflow which includes, among others, coverage of test costs by the insurance, involving relevant disciplines within the organization, etc.

Geisinger M edical Center introduced algorithms and warning systems in their service lines with the aim to reduce unnecessary blood component utilization for patients undergoing surgery (P-33). This involved several stakeholders at the hospital and served to increase effectiveness and efficiency of the patient care. 
This group is strongly related to the second category in Axis-1, i.e. Implementation Models. All the practices in category- 2 had a direct or indirect component of health care organizations, as summarized above.

\section{Box 6: Examples to practices serving to health professionals (group-3)}

\section{Mayo Clinic's Individualized Medicine Clinic (P-71)}

Two lines of services are introduced in this clinic: 1) tumor genomics services for patients with advanced cancer who fail standard therapy; 2) Whole Exome Sequencing and interpretation services for patients with a suspected genetic condition for whom previous genetic testing did not reveal an etiology. A common infrastructure for these was built and processes were designed with involvement of several disciplines and functions. These included IT, bioinformatics, bioethics, patient access management, education, revenue cycle, operations and the research sequencing facilities under the Individualized Medicine Clinic Working Group (USA) [75].

\section{Geisinger blood component utilization program (P-33)}

Geisinger M edical Center (USA) initiated a program with the overall goal to reduce unnecessary blood component utilization for patients undergoing surgery. Within this program, algorithms were developed to clarify when and which blood components to use and a system was set for communication between the pathologist and the patients' clinical team $7 / 24$, involving clinical pathologist when too much blood components were used for a given patient in the given time frame. The program was developed by the Transfusion Committee of the hospital [76].

\subsubsection{Group-4: Other organizations}

Three practices go beyond the borders of health care organizations. The first one eventually serves a national health system: the French $\mathrm{NCl}^{\prime}$ s nationwide program for tumor genomics (P-44) (see Box 2). The program aims not only increasing effectiveness and efficiency of cancer treatment, but also providing equitable services to the population. These correspond to the main goals of a health system (effectiveness, efficiency, patient and employee satisfaction and equity). Thus, PM/PHC becomes linked to tools/ways for health systems to perform better.

Second example is a health care payer organization: a social health insurance fund, Techniker Krankenkasse, in Germany, which implies that PM/PHC is not only for health care providers but also for payers. Within the 'Telemedicine for the Heart' program, telemedicine services for heart failure patients within their insured population were provided ( $\mathrm{P}$ 60) (see Box 7). In the case of the payer organization, PM/PHC becomes a tool in the service package of the payer organization to provide more efficient and effective services. 
Third practice is an international system of national and local antidoping agencies, as well as the World Anti-doping agency (P-42) (see Box 2).

\section{Box 7: Examples to practices serving to other organizations (group-4)}

\section{Telemedicine for the Heart (P-60)}

A telemedicine program which consists of nurse-calls to motivate patients to perform regular self-measurements (blood pressure, pulse, weight) and their recording was offered to the members of Techniker Krankenkasse, which is a sickness fund (social health insurance fund) in Germany. In the case the measured values are outside of set limits, calls to physicians were placed for therapy adjustments [77].

\subsection{Integration of practices into real life}

The two axes summarized above can give new perspectives on PM/PHC. However, they are not enough to provide a wider picture of issues around integration of these practices into real life (health) services. Are these practices available to everyone in their respective countries as commodities in the health care market (category-1)? Are the implementation models (category-2) available in all health institutions in a given country? Even if they are available, can the individuals access them without geographical, financial or other barriers? What about the situation globally?

It was not possible to get the answers to these questions for most of the practices because issues around availability and accessibility are not regularly reported in articles describing practices in the scientific literature. Moreover, many of these are inherent in the health system where the provider is placed in. Considering these, we chose three country examples among the practices in category-2: USA, France and Taiwan, which included some information on these dimensions.

\subsubsection{Cases: USA, France and Taiwan}

In category-2, (implementation models) we see that several medical centers (centers that combine health care service provision, research and medical education) and other major health care providers in USA have initiated some sort of a program or project on 'personalized', 'individualized', or 'precision' medicine in the last five years. These programs often involve a genetic component, such as tumor and cancer genomics and pharmacogenomics (P-25, P-30 [78], P-32, P-38 [79], P-41 [80], P-56 [81], P-57, P-61 [82], P-62, P-71, P-75 [83], P-77 [84], P-80 [85], P-82) (see Box 2 and Box 6). Many of the institutions initializing these projects are world-renowned major medical centers, which often pioneer introduction of innovations in mainstream medicine in the world. Many of their 'personalized' programs in category-2 are probably one of the first of their kind in USA or worldwide.

However, from 'availability' point of view, these 'personalized' programs are actually 'prestigious' services that are available to a very limited number of people who have geo- 
graphical and financial access to these major medical centers. For the rest of the U.S. population, the availability is unknown.

Moreover, the U.S. health system has serious problems with accessibility to health services. Uninsured and underinsured population and ineffectiveness of the insurance based health finance are major ones that leads to disparities in the system [86].

In France, the NCl's nationwide initiative provides tumor genetics/ genomics services to all cancer patients and is funded by the $\mathrm{NCl}$ and $\mathrm{M}$ inistry of Health (P-44, see Box 2). Thus, tumor genomics services are not limited to certain hospitals, or geographical regions and are available and accessible (geographically and financially) for all cancer patients who need it. This indicates "equity".

Third example is from Taiwan. A program called Ubiquitous Healthcare was developed and introduced at the National Taiwan University Hospital (P-24). It is a set of telehealth/tele-care solutions which aim to provide services to patients living in remote areas. In a sense, it is an attempt to overcome geographic accessibility problems, in the context of a country that embraced mobile technologies widely. However, the fact that this service is being promoted among the target group of patients by a marketing team suggests that it is to be paid out-of-pocket. The costs seem not to be extremely high (e.g. monthly cost of a cardiovascular patient is around 150 EUR). Nevertheless, for people from lower income groups, it can still be unaffordable. Thus, at this stage where it is not yet covered by the National Health Insurance of Taiwan, it is available only to people who has access to the university hospital at least once, and can afford it.

\section{Box 8: Example to a practice provided with out-of-pocket payment}

\section{Ubiquitous Healthcare (P-24)}

National Taiwan University Hospital (Taipei, Taiwan), combined the home healthcare service network and sensors network into a new service known as the Ubiquitous Healthcare (U-Health) service system in order to continually provide personalized healthcare services to patients who live in remote areas. The system included six service components that are introduced in an integrated manner: tele-education, tele-diagnosis, tele-monitoring, teleconsultation, tele-therapy and lifetime health. The services were targeted towards the following patient segments: post-discharge patients, patients suffering from cardiovascular diseases, patients suffering from chronic diseases and patients in hospice care. It reduced re-hospitalization rate and emergency room visits during the post-discharge period. [87] 


\subsubsection{Contextual factors: attitude towards innovations and health systems and policies}

These cases indicate that two factors, among others, may be important for integration of personalized practices in services: attitude of health care providers and society towards innovations and the health system and policy context.

USA has long been regarded as a global leader in medical technology innovation [88] and, as a matter of fact, PM/PHC have been closely linked to technological advancements. Apart from the publication tradition of these high profile centers in scientific journals in English; high number of publications from USA may give the impression that uptake of innovation is higher in USA. However, we don't know if these services are limited to these high profile centers, or widely available. There is a strikingly different example from the other side of the Atlantic: French NCl's initiative, making tumor genomics services available and accessible for everyone who needs it.

These contrasting cases illustrate that not only the attitude of the country towards health innovations, but also the health system and policy values play an important role in the uptake and implementation of personalized practices. French health system has 'equity' as a central policy value, reflected with universal health coverage for all citizens. In line with this, they provide this service in an equitable way to all citizens with a special program. On the other hand, a prominent policy value in U.S. health system is 'liberty', supporting the free market economy largely even in the health system, at cost of the equity [86, 89] (see also Chapter 3). Thus, prestigious high profile medical centers compete for newer and more innovative applications. Lack of equity as a policy value also results in a huge proportion of uninsured and underinsured population who cannot access health services. This means that, even if the individuals can geographically access the high-profile medical centers, they may not be able to access the 'prestigious' 'personalized' services due to their inability to pay for it.

We also see the effect of components of health systems on these examples. Such contextual issues are not explicitly addressed, but implied in a few of the articles. For example, some of the health care institutions from USA refer to 'insurance coverage' for the practices they offer [75, 82, 90, 91]. In the country context, this implies all the issues behind it within the U.S. health system, which create a barrier to operationalization of these services such as the difficulty of dealing with multiple payers (insurances) which decide independently on coverage of these new, not yet standardized, 'personalized' services. On the other hand, in a single payer health system, i.e. France, it was possible to cover the whole population for tumor genomics services with a special program and dedicated funds for it.

In the case of Taiwan, where out-of-pocket services account for almost $40 \%$ of the health care expenditure [92], we see that a tele-care practice enters the market as an out-ofpocket service. For some countries, it is customary for new technologies to enter the 
health care market as an out-of-pocket service and gradually become covered by third party payers. This means that the availability and accessibility of a practice in a given country can evolve with time.

\section{Discussion}

\subsection{Summary of the main findings}

In this study, a systematic search and inclusion strategy was used to identify 'personalized' practices within the scientific literature. Neither the terminology used to describe the practices (personalized, individualized, systems, medicine, health care, etc.) nor the technologies used showed a consistent pattern among the included practices. Thus a different angle grounded on the practices was taken to analyze them to reveal notions of PM/PHC.

The analysis of 'the application form and context' indicated that PM/PHC can be a commodity for the health market, a way in which health services are organized/provided, or 'future' or 'novel' emerging applications. When reviewing whom these practices were aiming to serve, we identified that PM/PHC can serve individuals (or patients) to empower them to gain control of their health, health professionals to help them provide 'even' better health services, health care organizations to help increasing the effectiveness/ efficiency of health care delivery in the competitive environment, or national health systems to help increasing their performance in terms of effectiveness/efficiency and equity. During the analysis it was noted that for successful integration of the practices into health services, not only the attitude of the country towards innovations, but also the health system and policies context is crucially important.

\subsection{Strengths and limitations}

This article aimed to investigate the field of "personalized" care from a practice-based perspective, not restricted by current (top-down) theoretical frameworks. Due to uniqueness of the research aim and heterogeneity of the included practices, a combination of methods was used at every step of analysis. Among these were: triangulation with different strategies applied by two independent researchers during the screening phase, utilization of the 'health intervention' definition of 'International Classification of Health Interventions (ICHI)' to assist the selection process, content analysis grounded on the data (practices/articles) for identification of relevant patterns. This combination of methods and tools allowed us to include a broad range of sectors/technologies and research/ development stages, which enabled us to demonstrate the spectrum of 'personalized' practices reported in the literature. To our best knowledge, this makes the paper unique in the field.

The main limitation of our study is that the search was carried out using the scientific literature alone without inclusion of information from web sites. As a consequence, some 
well-established existing practices may not have been included in the analysis. This restriction was deliberately made in order to ensure the quality and credibility of the practices analyzed.

Data on integration of practices into health services was very limited. Therefore, three prominent and contrasting country examples were identified to discuss some of the important factors that affect availability and accessibility. Since only the identified articles were analyzed, we weren't able to cross-check or elaborate on all aspects. Further research with a targeted approach using various other data sources can yield a more comprehensive analysis of the issues raised.

\subsection{Implications of the findings for the integration of personalized approaches}

Since early 2000s, there has been a growing expectation that 'personalized medicine' would enter the health services within a decade and gradually become common practice. In the early stages, it was mostly genomics that fueled these expectations, but later 'personalization' became a broader theme. However, as years proceeded, the genome-based applications did not enter routine practices as anticipated. This led to questioning whether personalization of medicine is a 'myth' rather than reality [93-95]. It is now expected that clear examples of application of genome-based information in medicine and health care may start to flourish in this decade, but will probably become more prominent in the period beyond the year 2020 [96]. The below discussion on the implications of our 'practicebased' findings for integration of PM/PHC may provide insights on some of the possible factors contributing to these unfulfilled claims. Among them are: excessive technological focus, issues with definitions, issues with the transformation of practices, and discrepancy between initial vision and practice.

\subsubsection{Excessive technological focus}

There is a striking contrast in terms of the technological orientation, in particular genomics, between the top-down descriptions and the findings of this study. The axes that emerged in our study didn't point technologies or methods to define PM/PHC. Instead, they reflected on some of the real life issues that practices face.

Many of the 'top-down' descriptions build the definition of the personalized approaches on the use genome-based technologies and molecular markers [12, 14, 15, 18]. However, many of the practices identified in this study didn't have a genomic or molecular component. This is illustrated in the Results section by giving at least two examples to each category and group: one that includes and one that doesn't include genomic/molecular technologies. Besides, there are some practices that have no technological component at all (e.g., P-12, P-81 [97], P-86, P-18 [98]). Thus, our findings indicate that it is not the use of certain technologies or methods, including genomic or molecular markers what makes PM/PHC 'personalized'. 
The problems around equating PM/PHC to genomics have already been raised. First of all, this causes attribution of currently unfulfilled expectations of genomic medicine to the broader application of PM/PHC [5]. Furthermore, genomic markers can be one of the components but is not the only factor that makes individuals unique or medicine personalized [2].

\subsubsection{The definitions}

The axes that emerged in this study were very different from the dimensions highlighted by the more common top-down definitions. However, the practices still did not yield a single common theme shared among them, which was also the case for top-down definitions as addressed in the Introduction. Our findings indicated that PM/PHC practices are present in several forms and contexts, serving a variety of groups, having different connotations in terms of availability and accessibility around the world. It is not a realistic expectation to have one definition to cover all of these. Is PM/PHC a commodity that can be bought or sold? Is it a tool to empower individuals to gain more control of their health? Is it a tool for health professionals to provide even better health care? Is it the way how health services are organized and provided? Is it something or anything novel (with or without genomics) that is emerging? Is it 'prestigious' applications in 'high-profile' clinics? All or none of these?

Thus, similar to the case of top-down definitions, a consistent and unified definition of the personalized approaches cannot be made from the practice-based perspective either. This means that people may presume that they talk about same concepts when they are using the same terms, while they actually have very different perceptions and ideas. This scene can be recognized by many people who try to follow the field via the literature or by participating in related meetings, in particular the multidisciplinary ones. Lack coherence in the use of concepts and terminology can be a significant factor contributing to the unfulfilled claims.

Looking at some specific aspects of existing definitions, the distinction made earlier between 'personalized medicine' and 'personalized health care' [5] seems to apply to the practices as well. The findings of the axis-1 indicated that having a product or service available for use in the market is one thing (category-1), and actually implementing it in a certain context is another (category-2).

The 'personalized health care' description $[5,23,99,100]$ seems to match with the category-2, which includes practices that are implemented in a certain context, mostly a health care provider setting. Typical examples of 'personalized health care' are My PREVENT Plan (P-49)[101] and Gentest (P-20), which use personal health profiles and 'personalized medicine tools' in a broader context to prepare personalized health plans (the former focus more on chronic disease management whereas the latter on their prevention). 
In the framework of Simmons et al., personalized health care is differentiated from the personalized medicine [5]. While the former is focused on a strategic approach to patient care, i.e. health services, the latter deals with the use of specific 'tools' in medicine. Further, 'personalized medicine' definition by Personalized Medicine Coalition, USA [15], has some sort of a match with category-1 in the sense that the Coalition also position personalized medicine as a commodity in health care market, but with particular focus on diagnostic tests and related drugs which involve genomic and molecular markers [102]. Most practices in category-1 are some sort of tests or drugs that are, however, not restricted to genomics or molecular markers.

On a map of the various concepts defining the personalized approaches, Pokorska-Bocci et al. depicted personalized health care as a superset of all of them [3]. This gives the impression that the difference is in their scope, but it is probably beyond that. There are two dimensions that distinguish them. First one is the application form and context, as supported with our findings and explained above (commodities/tools in the market vs. implementation model in a health care context). Second dimension is that personalized medicine is used to refer to specific tools, whereas personalized health care is used for a broader, holistic approach to health.

In brief, our findings support the strategy to use 'personalized medicine' for specific tools that are available for practice in medicine (such as tests, test-drug pairs, predictive algorithms, etc.), as in category-1, whereas refer to personalized health care for health services that have a holistic approach to the individual to be implemented in certain contexts.

\subsubsection{Transformation of practices}

$\mathrm{PM} / \mathrm{PHC}$ is a new and dynamic field, thus it is possible that the available approaches transform or converge from one category to another. For example, an implementation model designed by one institution (category-2) may become a solution available to other stakeholders (category-1), i.e. Genelnsight Suite (P-32), or a generic practice available within the health care market (category-1), i.e. Gentest (P-20). Practices in category-3 (emerging practices) might converge to those available on the market (category-1) or institutional models (category-2). It is possible that some will evolve in different ways that hasn't been identified in this study. A targeted research using different data sources can explore how the practices evolve and transform through time.

For example, integrative Personal Omics Profile (iPOP) (P-50) and an example of 'quantified self' movement by Smarr (P-59) are categorized as emerging practices (category-3) in axis-1. They are single cases that use longitudinal health data, in line with the P4 medicine vision of Leroy Hood [25]. A new initiative called 100K Wellness Project takes the P4 medicine vision to a larger scale longitudinal research project with a practice component, where health coaches are trained to guide the participating individuals to change their 
lifestyle based on available 'actionable' data [25]. If we apply the distinction between personalized medicine (tools/commodities) vs. personalized health care (implementation models) to this case, $100 \mathrm{~K}$ Wellness Project is essentially an example to personalized health care, indicating transition from P4 medicine towards P4 health care.

Such transformations indicate the highly dynamic nature of the field, which is making the definitions more difficult. Moreover, by the time one definition framework is agreed on, it runs a risk to become outdated as new advances have emerged.

\subsubsection{Discrepancy between initial descriptions and practice}

One of the factors that contribute to why the field of PM/PHC hasn't fulfilled the expectations might be how PM/PHC was envisaged and defined in the first place. The top-down descriptions focus on the theoretical promises of available and forthcoming technologies and try to guide the field in that direction. They were designed mostly by highly influential individuals and committees on the science and technology side and assume that if the technology alone is good enough, it can solve the problems of today's health care. For example, P4 medicine was proposed to eventually lead to a universal democratization of health care by increasing effectiveness of preventive and curative interventions, and reducing the costs, thus lessening the social and financial burden of diseases $[19,25]$.

There is clearly a difference between how the field is depicted in these theoretical models and real life as reflected in the practices we identified and analyzed. In real life, innovations do not diffuse and enter services just because they are technically possible [103]. Policy makers, practitioners and/or individuals do not (decide to) use tools just because they exist. In line with the authors such as Bijker et al. [104] and Berg and Mol [105], development and integration of new practices are much more complex than the science and technology alone. It involves people, functions and bodies in health services in the whole context of health system, in interaction with the society.

Health practice side of the coin has been widely neglected. Practitioners are not 'empty vessels', who only absorb all the literature, guidance and visions set forth by the commissions and opinion leaders. As Green states, they are "... full of prior knowledge, attitudes, beliefs, values and, above all, contextual constraints at any given point in practice time" [27]. Practitioners adjust methods and technologies they use in order to successfully adapt them to the context, which inevitably changes and transforms them [105]. At the end of the day, it will be those health care practices, which will actually construct the field and shape how personalized approaches will actually take place in medicine and health care.

Health systems and policies context has also been underrepresented. For example, can inequity in a health system be solved by personalized practices alone? As seen in the case examples of USA, France and Taiwan, strengths and weaknesses of health systems have direct implications on the personalized practices. The practice cannot be isolated from the 
context. Moreover, issues related to health systems cannot be solved in isolation either. Various publications have discussed and reported the barriers, challenges or agendas for/ of personalized medicine. They list a number of items that need to be solved, such as reimbursement, data issues, physician education, and others, and try to tackle each individually, one at a time, independent from each other and the general context. This isolated and piecemeal approach is unlikely to solve the real life problems efficiently because they are all interlinked with each other and the context. Health system must be addressed in its entirety.

\subsection{Proposed solution: towards a true multi-disciplinary ap- proach in the field}

Realization of the PM/PHC depends on science, technology, health care and society domains [106]. Health care domain includes not only the health professionals who are in the front line of health services, but also other professionals, functions and bodies that make health care services available within local, regional and national context, thus covering both health policy and practice. When defining and working towards PM/PHC, predominance of specific domains hinders the progress by creating unrealistic expectations. Instead, the field must be created with a collaborative effort of all domains.

These efforts should not be limited to higher-level meetings, where academicians assemble and discuss the topics. To bring PM/PHC into practice, these domains must come together effectively in teams working on concrete projects. An inspiring example relevant to this field is the Institute for Systems Biology (ISB) in USA. Established in 2000, co-founded by Hood, ISB has been pioneering the field of systems biology. To study complex biological systems in a holistic way, a cross-disciplinary culture of research which uses technology to solve biological questions was required. For that, ISB created a special and unusual institutional platform, where scientists from different disciplines learn one another's languages and work effectively in teams, instead of disciplinary departments [107].

To bring PM/PHC to life, we also need an integrative 'systems' approach. The system here is much broader than the organism. It includes science, technology, health and society domains. Just like the interaction of genes, lifestyle and environmental factors, socioeconomic factors and health services, which determine health and disease, individuals from different disciplines and sectors need to come together, interact with each other and form real collaborations to realize PM/PHC. Various mechanisms can be used to facilitate this, such as double appointments in science, policy and practice, financial incentives to make different parties work together, and events across domains to trigger cross-fertilization. In terms of facilitating structures, ISB can be an inspiring example. In addition, education of health professionals (under and postgraduate), in particular physicians, is crucially important for them to be able to adopt innovations in practice. 
PM/PHC can fulfill its promises only if it is tackled in a collective way. If the field focuses only on science and technology, it can make significant discoveries and perhaps bring out tools for the health care market, but their real life use will be questionable.

\section{Conclusion}

This work presents an important endeavor of looking at the practice side of the coin of PM/PHC. For the first time, such analysis was based on empirical data in such an extensive way. The perspective presented in this article, methods used and the findings may trigger discussions on new ways to describe the personalized approaches. The practices identified and earlier publications show that personalized medicine and personalized health care are different entities, one involving commodities and tools available in health care market and the other involving holistic approaches to individuals and their implementation in actual settings. Science or technology alone is not enough to have an impact on the health of individuals. Health policy and practice side of the coin must also be taken into account to address innovation for health in its entirety. For that, science, technology, health care (including policy and practice) and society domains should work collectively towards realization of PM/PHC.

\section{Acknowledgments}

We thank Dr. Serdar Savas for his feedback on the manuscript, in particular on health systems related issues.

\section{Competing Interests}

I have read the journal's policy and the authors of this manuscript have the following competing interests: TC is among the developers of Gentest, which is among the included practices as a result of the systematic search and inclusion process.

\section{Supplementary files}

Supplementary material 1. Detailed description of the search and selection process

Supplementary material 2. Lists of practices identified (Tables S1, S2, S3, S4 and S5 summarizes the practices identified as a result of the systematic literature search.) 


\section{References}

1. Individualized Medicine at M eSH (Medical Subject Headings) Bethesda MD, USA: National Center for Biotechnology Information, U.S. National Library of Medicine; [20.06.2014]. Available from: http:// www.ncbi.nlm.nih.gov/mesh/?term=personalized +medicine.

2. Cesuroglu $T$, van Ommen B, Malats $N$, Sudbrak $R$, Lehrach $H$, Brand A. Public health perspective: from personalized medicine to personal health. Personalized Medicine. 2012 2012/03/01;9(2):115-9.

3. Pokorska-Bocci A, Stewart A, Sagoo GS, Hall A, Kroese M, Burton H. 'Personalized medicine': what's in a name? Personalized Medicine. 2014 2014/03/01;11(2):197-210.

4. Schleidgen S, Klingler C, Bertram T, Rogowski WH, Marckmann G. What is personalized medicine: sharpening a vague term based on a systematic literature review. BMC Med Ethics. 2013;14:55.

5. Simmons LA, Dinan MA, Robinson TJ, Snyderman R. Personalized medicine is more than genomic medicine: confusion over terminology impedes progress towards personalized healthcare. Personalized M edicine. 2011 2012/01/01;9(1):85-91.

6. ESF Forward Look - Personalised Medicine for the European Citizen. Strasbourg, France: European Science Foundation; 2012. 61 p.

7. Redekop WK, Mladsi D. The faces of personalized medicine: a framework for understanding its meaning and scope. Value in health : the journal of the International Society for Pharmacoeconomics and Outcomes Research. 2013 Sep-Oct;16(6 Suppl):S4-9.

8. Roden DM, Tyndale RF. Genomic medicine, precision medicine, personalized medicine: what's in a name? Clinical pharmacology and therapeutics. 2013 Aug;94(2):169-72.

9. Siest G. Systems medicine, stratified medicine, personalized medicine but not precision medicine. Drug metabolism and drug interactions. 2014;29(1):1-2.

10. Lopez-Campos GH, Lopez-Alonso V, M artin-Sanchez F. Is Precision Medicine different from Personalised Medicine? A Biomedical informatics perspective. Stud Health Technol Inform. 2014;202:20-3.

11. National Research Council (US) Committee on A Framework for Developing a New Taxonomy of Disease. Toward Precision Medicine: Building a Knowledge Network for Biomedical Research and a New Taxonomy of Disease. Washington, DC, USA: The National Academies Press (US); 2011.

12. President's Council of Advisors on Science and Technology (US). Priorities for Personalized Medicine. Washington, D.C.: Office of Science and Technology Policy, Executive Office of the President $\quad 2008.2$ Available from: http://www.whitehouse.gov/files/documents/ostp/PCAST/pcast_report_v2.pdf.

13. United States Department of Health and Human Services. Personalized Health Care: Opportunities, Pathways, Resources 2007. Available from: http:// www.genome.gov/Pages/Policy/PersonalizedM edicine/DHHSReportPersonalizedHealth. pdf.

14. Stratification biomarkers in personalised medicine (Summary Report of the "Workshop to clarify the scope for stratification biomarkers and to identify bottlenecks in the discovery and the use of such biomarkers", European Commission, Brussels, 10-11 June 2010). Brussels: European Commission, DG Research; 2010. Available from: http://ec.europa.eu/research/health/pdf/ biomarkers-for-patient-stratification_en.pdf.

15. Abrahams E, Ginsburg GS, Silver M. The Personalized Medicine Coalition: goals and strategies. American journal of pharmacogenomics : genomics-related research in drug development and clinical practice. 2005;5(6):345-55. 
16. CASyM Europe - Coordinating Action Systems Medicine: Implementation of Systems Medicine accross Europe. CASyM Report: 1sr CASyM open stakeholder consultation conference, Lyon, March 2013: CASyM administrative office, Project Management Jülich, Forschungszentrum Jülich GmbH, Germany; [updated August 2013].

17. Bousquet J, Anto JM, Sterk PJ, Adcock IM, Chung KF, Roca J, et al. Systems medicine and integrated care to combat chronic noncommunicable diseases. Genome M ed. 2011;3(7):43.

18. Hood L. A personal journey of discovery: developing technology and changing biology. Annual review of analytical chemistry (Palo Alto, Calif). 2008;1:1-43.

19. Hood L. A Doctor's Vision of the Future of M edicine. Newsweek. 2009 Jul 13, 2009.

20. Topol EJ. Transforming Medicine via Digital Innovation. Sci Transl Med. 2010 January 27, 2010;2(16):16cm4.

21. Topol E. The Creative Destruction of Medicine: How the Digital Revolution Will Create Better Health Care New York, NY: Basic Books; 2012.

22. Snyderman R, Williams RS. Prospective medicine: the next health care transformation. Acad Med. 2003 Nov;78(11):1079-84.

23. Snyderman R. Personalized health care: from theory to practice. Biotechnology journal. 2012 Aug; 7(8):973-9.

24. Bijker WE, Hughes TP, Pinch T, editors. The Social Construction of Technological Systems. anniversary ed. Boston, MA: The MIT Press; 2012.

25. Hood L, Price ND. Demystifying Disease, Democratizing Health Care. Sci Transl M ed. 2014 February 26, 2014;6(225):225ed5.

26. Cesuroglu T, Karaca S, Erge S. A practice model for personalized healthcare with a public health genomics perspective. Personalized Medicine.

27. Green LW. Making research relevant: if it is an evidence-based practice, where's the practice-based evidence? Family Practice. 2008 December 1, 2008;25(suppl 1):i20-i4.

28. Moher D, Liberati A, Tetzlaff J, Altman DG, Group P. Preferred reporting items for systematic reviews and meta-analyses: the PRISMA statement. PLoS Med. 2009 Jul 21;6(7):e1000097.

29. WHO-FIC Family Development Committee. International Classification of Health Interventions (ICHI) - alpha version: World Health Organization; 2012. Available from: http://sydney.edu.au/health-sciences/ncch/docs/ICHI-alpha-version2012.pdf.

30. Aronson SJ, Clark EH, Babb LJ, Baxter S, Farwell LM, Funke BH, et al. The Genelnsight Suite: a platform to support laboratory and provider use of DNA-based genetic testing. Human mutation. 2011 May;32(5):532-6.

31. Donnan JR, Ungar WJ, Mathews M, Hancock-Howard RL, Rahman P. A cost effectiveness analysis of thiopurine methyltransferase testing for guiding 6-mercaptopurine dosing in children with acute lymphoblastic leukemia. Pediatric blood \& cancer. 2011 Aug;57(2):231-9.

32. Babic N. Clinical pharmacogenomics and concept of personalized medicine. Journal of Medical Biochemistry. 201201 Oct;31(4):281-6.

33. Cohen J, Wilson A, Manzolillo K. Clinical and economic challenges facing pharmacogenomics. The pharmacogenomics journal. 2013 Aug;13(4):378-88.

34. Faruki $\mathrm{H}$, Lai-Goldman M. Application of a pharmacogenetic test adoption model to six oncology biomarkers. Personalized M edicine. 2010 July; 7(4):441-50. 
35. M ini E, Nobili S. Pharmacogenetics: implementing personalized medicine. Clinical cases in mineral and bone metabolism : the official journal of the Italian Society of Osteoporosis, Mineral M etabolism, and Skeletal Diseases. 2009 Jan;6(1):17-24.

36. Ritchie MD. The success of pharmacogenomics in moving genetic association studies from bench to bedside: study design and implementation of precision medicine in the post-GWAS era. Human genetics. 2012 Oct;131(10):1615-26.

37. Topol EJ, Lauer MS. The rudimentary phase of personalised medicine: coronary risk scores. Lancet. 2003 Nov 29;362(9398):1776-7.

38. Gottesman O, Scott SA, Ellis SB, Overby CL, Ludtke A, Hulot JS, et al. The CLIPM ERGE PGx Program: clinical implementation of personalized medicine through electronic health records and genomics-pharmacogenomics. Clinical pharmacology and therapeutics. 2013 Aug;94(2):214-7.

39. Pulley JM, Denny JC, Peterson JF, Bernard GR, Vnencak-Jones CL, Ramirez AH, et al. Operational implementation of prospective genotyping for personalized medicine: the design of the Vanderbilt PREDICT project. Clinical pharmacology and therapeutics. 2012 Jul;92(1):87-95.

40. Weitzel KW, Elsey AR, Langaee TY, Burkley B, Nessl DR, Obeng AO, et al. Clinical pharmacogenetics implementation: Approaches, successes, and challenges. American journal of medical genetics Part C, Seminars in medical genetics. 2014 M ar;166(1):56-67.

41. O'Donnell PH, Danahey K, Jacobs M, Wadhwa NR, Yuen S, Bush A, et al. Adoption of a clinical pharmacogenomics implementation program during outpatient care-initial results of the University of Chicago "1,200 Patients Project". American journal of medical genetics Part C, Seminars in medical genetics. $2014 \mathrm{Mar} ; 166(1): 68-75$.

42. Dolan ME, Maitland ML, O'Donnell PH, Nakamura Y, Cox NJ, Ratain MJ. Institutional Profile: University of Chicago Center for Personalized Therapeutics: research, education and implementation science. Pharmacogenomics. 2013 Sep;14(12):1383-7.

43. Gharani N, Keller MA, Stack CB, Hodges LM, Schmidlen TJ, Lynch DE, et al. The Coriell personalized medicine collaborative pharmacogenomics appraisal, evidence scoring and interpretation system. Genome medicine. 2013 Oct 18;5(10):93.

44. Huang RS, Gamazon ER. Translating pharmacogenomics discoveries into the clinic: an implementation framework. Genome medicine. 2013 Oct 18;5(10):94.

45. Bielinski SJ, Olson JE, Pathak J, Weinshilboum RM, Wang L, Lyke KJ, et al. Preemptive genotyping for personalized medicine: design of the right drug, right dose, right time-using genomic data to individualize treatment protocol. M ayo Clinic proceedings. 2014 Jan;89(1):25-33.

46. Mascarin M, Truccolo I, Byther E, Capone D, Elia C. Cancer, Adolescence, and Their Peers: "They'll give you a Story". Journal of cancer education : the official journal of the American Association for Cancer Education. 2014 Mar 18.

47. Andre F, Nowak F, Arnedos M, Lacroix L, Viens P, Calvo F. Biomarker discovery, development, and implementation in France: a report from the French National Cancer Institute and cooperative groups. Clinical cancer research : an official journal of the American Association for Cancer Research. 2012 Mar 15;18(6):1555-60.

48. Sottas PE, Robinson N, Rabin 0 , Saugy $M$. The athlete biological passport. Clinical chemistry. 2011 Jul;57(7):969-76.

49. Chun H, Kang J, Kim KJ, Park KS, Kim HC. IT-based diagnostic instrumentation systems for personalized healthcare services. Studies in health technology and informatics. 2005;117:180-90.

50. Li A, Bozdag S, Kotliarov Y, Fine HA. GliomaPredict: a clinically useful tool for assigning glioma patients to specific molecular subtypes. BMC medical informatics and decision making. 2010;10:38. 
51. Boudreaux ED, Bedek KL, Byrne NJ, Baumann BM, Lord SA, Grissom G. The ComputerAssisted Brief Intervention for Tobacco (CABIT) program: a pilot study. Journal of medical Internet research. 2012;14(6):e163.

52. Laerum H, Bremer S, Bergan S, Grunfeld T. A taste of individualized medicine: physicians' reactions to automated genetic interpretations. Journal of the American Medical Informatics Association : JAM IA. 2014 Feb;21(e1):e143-6.

53. Chen R, Mias Gl, Li-Pook-Than J, Jiang L, Lam HY, Chen R, et al. Personal omics profiling reveals dynamic molecular and medical phenotypes. Cell. 2012 M ar 16;148(6):1293-307.

54. Smarr L. Quantifying your body: a how-to guide from a systems biology perspective. Biotechnology journal. 2012 Aug;7(8):980-91.

55. Arribas-Ayllon M, Sarangi S, Clarke A. Promissory accounts of personalisation in the commercialisation of genomic knowledge. Communication \& medicine. 2011;8(1):53-66.

56. Palmer JE. Genetic gatekeepers: regulating direct-to-consumer genomic services in an era of participatory medicine. Food and drug law journal. 2012;67(4):475-524, iii.

57. Platt D. When consumers get their genomes. Personalized M edicine. 2009;6(6):669-79.

58. Richards M. Reading the runes of my genome: A personal exploration of retail genetics. New Genetics and Society. 2010 September;29(3):291-310.

59. Tenenbaum JD, James A, Paulyson-Nunez K. An altered treatment plan based on direct to consumer (DTC) genetic testing: Personalized medicine from the patient/pin-cushion perspective. Journal of Personalized M edicine. 2012 December;2(4):192-200.

60. Inspections, Compliance, Enforcement, and Criminal Investigations - 23andMe, Inc. 11/22/13: U.S. Food and Drug Administration; 2013 [updated 25.11.201321.12.2013]. Available from: http://www.fda.gov/iceci/enforcementactions/warningletters/2013/ucm376296.htm.

61. 23ANDME, INC. PROVIDES UPDATE ON FDA REGULATORY REVIEW: 23andMe inc.; 2013 [updated 5.12.201321.12.2013]. Available from: http://mediacenter.23andme.com/pressreleases/23andme-inc-provides-update-on-fda-regulatory-review/.

62. Wac K. Smartphone as a personal, pervasive health informatics services platform: literature review. Yearbook of medical informatics. 2012;7(1):83-93.

63. Andre F, Ciccolini J, Spano JP, Penault-Llorca F, Mounier N, Freyer G, et al. Personalized medicine in oncology: where have we come from and where are we going? Pharmacogenomics. 2013 Jun;14(8):931-9.

64. Arnedos M, Vielh P, Soria JC, Andre F. The genetic complexity of common cancers and the promise of personalized medicine: is there any hope? The Journal of pathology. 2014 Jan;232(2):274-82.

65. Bailey AM, Mao Y, Zeng J, Holla V, Johnson A, Brusco L, et al. Implementation of biomarker-driven cancer therapy: existing tools and remaining gaps. Discovery medicine. 2014 Feb;17(92):101-14.

66. Cheng S, Koch WH, Wu L. Co-development of a companion diagnostic for targeted cancer therapy. New biotechnology. 2012 Sep 15;29(6):682-8.

67. Donnelly E. XalKori: A new paradigm of therapeutic and companion diagnostic codevelopment. Regulatory Rapporteur. 2013;10(2):9-11.

68. Van Allen EM, Foye A, Wagle N, Kim W, Carter SL, McKenna A, et al. Successful wholeexome sequencing from a prostate cancer bone metastasis biopsy. Prostate Cancer and Prostatic Diseases. 2014 M arch;17(1):23-7. 
69. Waring PM. Matching patients with drugs: Triumphs and challenges. Personalized Medicine. 2006 August;3(3):335-44.

70. Ong FS, Das K, Wang J, Vakil H, Kuo JZ, Blackwell WL, et al. Personalized medicine and pharmacogenetic biomarkers: progress in molecular oncology testing. Expert review of molecular diagnostics. 2012 Jul; 12(6):593-602.

71. Love D, Stratton E, Stocum M. Best practices for companion diagnostic and therapeutic development: Translating between the stakeholders. New Biotechnology. 201215 Sep;29(6):689-94.

72. Leopold C, Vogler S, Habl C, Mantel-Teeuwisse AK, Espin J. Personalised medicine as a challenge for public pricing and reimbursement authorities - A survey among 27 European countries on the example of trastuzumab. Health policy (Amsterdam, Netherlands). 2013 Dec;113(3):313-22.

73. Ou SH, Bartlett CH, M ino-Kenudson M, Cui J, lafrate AJ. Crizotinib for the treatment of ALKrearranged non-small cell lung cancer: a success story to usher in the second decade of molecular targeted therapy in oncology. The oncologist. 2012;17(11):1351-75.

74. Kondylakis H, Kazantzaki E, Koumakis L, Genitsaridi I, Marias K, Gorini A, et al. Development of interactive empowerment services in support of personalised medicine. Ecancermedicalscience. 2014;8:400.

75. Lazaridis KN, McAllister TM, Babovic-Vuksanovic D, Beck SA, Borad MJ, Bryce AH, et al. Implementing individualized medicine into the medical practice. American journal of medical genetics Part C, Seminars in medical genetics. 2014 Mar;166(1):15-23.

76. Brown RE, Dorion RP, Trowbridge C, Stammers AH, Fitt W, Davis J. Algorithmic and consultative integration of transfusion medicine and coagulation: a personalized medicine approach with reduced blood component utilization. Annals of clinical and laboratory science. 2011 Summer;41(3):211-6.

77. Sohn S, Helms TM, Pelleter JT, Muller A, Krottinger Al, Schoffski O. Costs and benefits of personalized healthcare for patients with chronic heart failure in the care and education program "Telemedicine for the Heart". Telemedicine journal and e-health : the official journal of the American Telemedicine Association. 2012 Apr;18(3):198-204.

78. MacDonald DJ, Blazer KR, Weitzel JN. Extending comprehensive cancer center expertise in clinical cancer genetics and genomics to diverse communities: the power of partnership. Journal of the National Comprehensive Cancer Network : JNCCN. 2010 M ay;8(5):615-24.

79. Khan NA, Peterson JF. A surveillance tool to support quality assurance and research in personalized medicine. AMIA Annual Symposium proceedings / AMIA Symposium AMIA Symposium. 2011;2011:701-8.

80. Roychowdhury S, lyer MK, Robinson DR, Lonigro RJ, Wu Y-M, Cao X, et al. Personalized Oncology Through Integrative High-Throughput Sequencing: A Pilot Study. Science Translational Medicine. 2011 November 30, 2011;3(111):111ra21.

81. Printz C. Universities bring personalized medicine to the forefront of patient care: two programs aim to make customized treatments easily accessible. Cancer. 2012 M ay 15;118(10):25634.

82. Teng K, Eng C, Hess CA, Holt MA, Moran RT, Sharp RR, et al. Building an innovative model for personalized healthcare. Cleveland Clinic Journal of M edicine. 2012 Apr;79 Suppl 1:S1-9.

83. Meric-Bernstam F, Farhangfar C, M endelsohn J, Mills GB. Building a personalized medicine infrastructure at a major cancer center. Journal of clinical oncology : official journal of the American Society of Clinical Oncology. 2013 M ay 20;31(15):1849-57.

84. Patel CJ, Sivadas A, Tabassum R, Preeprem T, Zhao J, Arafat D, et al. Whole genome sequencing in support of wellness and health maintenance. Genome M ed. 2013 Jun 27;5(6):58. 
85. Vorderstrasse AA, Ginsburg GS, Kraus WE, Maldonado MC, Wolever RQ. Health coaching and genomics-potential avenues to elicit behavior change in those at risk for chronic disease: protocol for personalized medicine effectiveness study in air force primary care. Global advances in health and medicine : improving healthcare outcomes worldwide. $2013 \mathrm{M}$ ay;2(3):26-38.

86. Savas BS, Cesuroglu T. Health for America: European Arguments for a Paradigm Shift. In: Shah A, Colombano J, editors. Learning from the World: New Ideas to Redevelop America Palgrave Macmillan; 2013. p. 167-84.

87. Chang HL, Shaw MJ, Lai F, Ko WJ, Ho YL, Chen HS, et al. U-Health: An example of a highquality individualized healthcare service. Personalized M edicine. 2010 November;7(6):677-87.

88. PricewaterhouseCoopers. PwC Medical Technology Innovation Scorecard Highlights: PricewaterhouseCoopers; 2013 [14.11.2014]. Available from: www.pwc.com/InnovationScorecard.

89. Wang XL, Gui Q, Liu BW, Jin ZP, Chen Y. Enabling Smart Personalized Healthcare: A Hybrid Mobile-Cloud Approach for ECG Telemonitoring. IEEE journal of biomedical and health informatics. 2014 May;18(3):739-45.

90. Farrugia G, Weinshilboum RM . Challenges in implementing genomic medicine: the Mayo Clinic Center for Individualized Medicine. Clinical pharmacology and therapeutics. 2013 Aug;94(2):204-6.

91. McClure JB, Swan GE, St John J, Fauver R, Javitz HS, Bergen AW, et al. Pharmacogenetic smoking cessation intervention in a health care setting: a pilot feasibility study. Nicotine $\&$ tobacco research : official journal of the Society for Research on Nicotine and Tobacco. 2013 Feb;15(2):51826.

92. Checking up on Taiwan healthcare - Market challenges and opportunities: PricewaterhouseCoopers Taiwan; 2012 [18.12.2014]. Available from: https://www.pwc.tw/en_Tw/tw/industries/publications/assets/healthcare-en.pdf

93. Dabrock P, Braun M, Ried J. Individualized Medicine Between Hype and Hope: Exploring Ethical and Societal Challenges for Healthcare. Münster: LIT Verlag M unster; 2012.

94. Chabner BA, Ellisen LW, lafrate AJ. Personalized Medicine: Hype or Reality. The Oncologist. 2013 June 1, 2013;18(6):640-3.

95. Greenberg T. Conference Scene: Personalized Medicine comes to Harvard. Personalized M edicine. 2011 2012/01/01;9(1):17-8.

96. Green ED, Guyer MS. Charting a course for genomic medicine from base pairs to bedside. Nature. 2011 Feb 10;470(7333):204-13.

97. Barnard KD, Lloyd CE, Dyson PA, Davies MJ, O'Neil S, Kamunilli N, et al. Kaleidoscope model of diabetes care: time for a rethink? Diabet M ed. 2014 Feb 7.

98. Kazak AE. Pediatric Psychosocial Preventative Health Model (PPPHM): Research, practice, and collaboration in pediatric family systems medicine. Families, Systems and Health. 2006 Winter;24(4):381-95.

99. Simmons LA, Wolever RQ, Bechard EM, Snyderman R. Patient engagement as a risk factor in personalized health care: a systematic review of the literature on chronic disease. Genome Med. 2014 Feb 26;6(2):16.

100. Snyderman R, Yoediono Z. Perspective: Prospective health care and the role of academic medicine: lead, follow, or get out of the way. Acad Med. 2008 Aug;83(8):707-14.

101. Burnette R, Simmons LA, Snyderman R. Personalized health care as a pathway for the adoption of genomic medicine. Journal of personalized medicine. 2012 December;2(4):232-40. 
102. Personalized Medicine Coalition. The case for personalized medicine (4th Edition). Washington D.C. (US): PMC; 2014 25.06.2015]. Available from: http://www.personalizedmedicinecoalition.org/Resources/The_Case_for_Personalized_M edicine.

103. Rogers EM . Diffusion of Innovations. Third ed. NY, USA: The Free Press; 1983. 453 p.

104. Bijker WE. How is technology made?-That is the question! Cambridge Journal of Economics. 2009 January 1, 2009.

105. Berg M, Mol A, editors. Differences in Medicine: Unraveling Practices, Techniques, and Bodies. Durham, NC and London: Duke University Press,; 1998.

106. Periodic Report of P4-MEDICINE@EU project - Evaluation of a Best Practice Model for Personalized Health Care within Public Health Genomics (Project No.:272563). Submitted to the European Commission, Research Executive Agency, Marie Curie Actions (Intra-European Fellowships for Career Development) on 05.06.2014. 2014. Available from: Report Summary http://cordis. europa.eu/result/rcn/150016_en.html.

107. Hood L. Systems biology and p4 medicine: past, present, and future. Rambam Maimonides medical journal. $2013 \mathrm{Apr}$;(2):e0012. 


\section{Supplementary Material 1 \\ Detailed description of the search and selection process}

\section{Choice of databases}

We searched for journals that include 'personalized' or 'personalised' in their title or description in National Library of M edicine Catalog as well as Google.

- NLM Catalog search (18.03.2014) with the terms "personalized medicine journal" and "personalised medicine journal"

- Google search (18.03.2014) with the term "journal personalized medicine"

We identified 9 scientific journals with these terms in their name or description. By checking the journal web sites and, when necessary, databases, we listed where each journal is indexed. We identified that among the database combinations, PubMed and EMBASE together would give the maximum number of journals covered for the search (8), and therefore decided to choose them. The journals included in either of those databases are:

- $\quad$ Current Pharmacogenomics and Personalized Medicine (Bentham Science, Dubai)

- $\quad$ EPM A Journal (BioM ed Central)

- Journal of Personalized M edicine (MDPI, Basel)

- Journal of Translational M edicine (BioM ed Central)

- $\quad$ Personalized M edicine (Future Medicine, London)

- $\quad$ Personalized Medicine Universe (Elsevier)

- $\quad$ Pharmacogenomics and Personalized Medicine (Dove Press, New Zealand)

- Mount Sinai Journal of Medicine: A Journal of Translational and Personalized Medicine (Wiley)

Only one journal, International Journal of Functional Informatics and Personalised Medicine (InderScience, UK) is indexed in neither PubM ed, nor EM BASE.

\section{Identification}

PubMed was searched on 1 April 2014 via PubMed.gov and EM BASE on 14 April 2014 via OVID. The following search string was used in both:

\footnotetext{
"personalized health care" OR "personalized healthcare" OR "personalized medicine" OR "P4 medicine" OR "precision medicine" OR "stratified medicine" OR "systems biomedicine" OR "systems medicine" OR "individualized medicine" OR "personalised health care" OR "personalised healthcare" OR "personalised medicine" OR "individualised medicine"
}

In both databases the title or abstract was searched, limited to English articles, without time limits. 
The search at PubMed gave 4.317 records and EM BASE 6.328, all of which were imported to EndNote. By using different strategies in EndNote (automated, half automated and manual) 4.063 duplicates were removed. It is of note that in this very large set of records, duplicates were present not only between databases, but also within databases. Records of meeting abstracts were also removed since there is no full-text available for them and 5.333 records remained.

Inclusion criteria: Our inclusion criteria was "health intervention that is developed (designed) and/or implemented and presented in association with one of the terms used to describe the personalized vision in medicine and health care (see the search terms)". To be more clear in our inclusion criteria, we looked for health intervention/ practices which were implemented/demonstrated on at least one case (it can be as an example or a pilot; a drug and test on the market; a short test to assess feasibility, etc.) and had enough details provided in the relevant article(s). These criteria applied to both Screening and Eligibility stages.

\section{Screening}

Screening was carried out by two independent researchers, with two different strategies. First researcher (TC) screened all of the 5.333 titles and, as necessary, abstracts.

For triangulation, second researcher (ES) applied a different strategy: she made a second search within the database of 5.333 records using a large list of terms that are potentially relevant for identifying the practices and screened titles and abstracts of the records revealed by this search. The second search terms included the following:

\footnotetext{
"analytical model", "application", "case study", "clinic", "clinical”, "clinical practice", "clinical AND evaluate", "clinical AND translation", "education", "evaluate", "evaluation", "implement", "implementation", "innovat", "innovation", "introduce", "introduction", "introduction AND clinic", "model", "patient", "patient data", "pilot", "practical", "practice", "real-life", "testing", "tool", "transition", "translate", "translation", "valorization"
}

The two researchers compared and discussed their results and reached a consensus for inclusion of 277 records in the screening phase. Full text manuscripts were obtained for 262 records (95\% of the included ones).

\section{Eligibility (1 ${ }^{\text {st }}$ stage)}

Articles were reviewed using the inclusion criteria as set above by two researchers (TC and ES) independently. They compared their results, had a discussion and reached a consensus to include full text of 179 articles in the analysis. The reasons for exclusion (83 records) are presented below: 
- $\quad$ not implemented/presented on a case: 44

- $\quad$ not 'health intervention': 14

- $\quad$ not associated with 'personalized medicine' (or other keywords searched): 2

- $\quad$ research and development tool or strategy: 14

- $\quad$ review of gene-disease or gene-drug associations: 2

- $\quad$ not enough details: 7

Some articles could have been excluded for more than one reason from the above list, but it was counted only for the most apparent reason.

\section{Additional references}

While the full texts were investigated, additional records were identified from the references. They were included only if they were present in the first set of 5.333 records and complied with the inclusion criteria. This brought 5 additional references, leading to 184 included items.

\section{Eligibility (2 ${ }^{\text {nd }}$ stage)}

While specific practices were determined from the articles (see below) it was identified that 27 articles didn't actually meet the inclusion criteria and therefore excluded (see Figure 1). The reasons for exclusion (27 records) are presented below:

- $\quad$ - not implemented/presented on a case: 16

- $\quad$ - not 'health intervention': 1

- $\quad$ not enough details: 10

Eventually, 157 articles were included.

For the flow diagram, please refer to Figure 1 of the article. 


\section{Supplementary Material 2 Lists of practices identified}

This material contains the tables that list, summarize and present the properties of the practices identified as a result of the systematic literature search. The articles referred in the tables are listed at the end under 'references'. Table S1 to S5 summarizes the practices identified as a result of the systematic literature search.

\section{List of tables:}

Table S1: Basic properties of included practices

Table S2: Details of grouped practices and their references

Table S3: Practices available in health care market (Category-1)

Table S4: Implementation models (Category-2)

Table S5: Emerging practices (Category-3) 

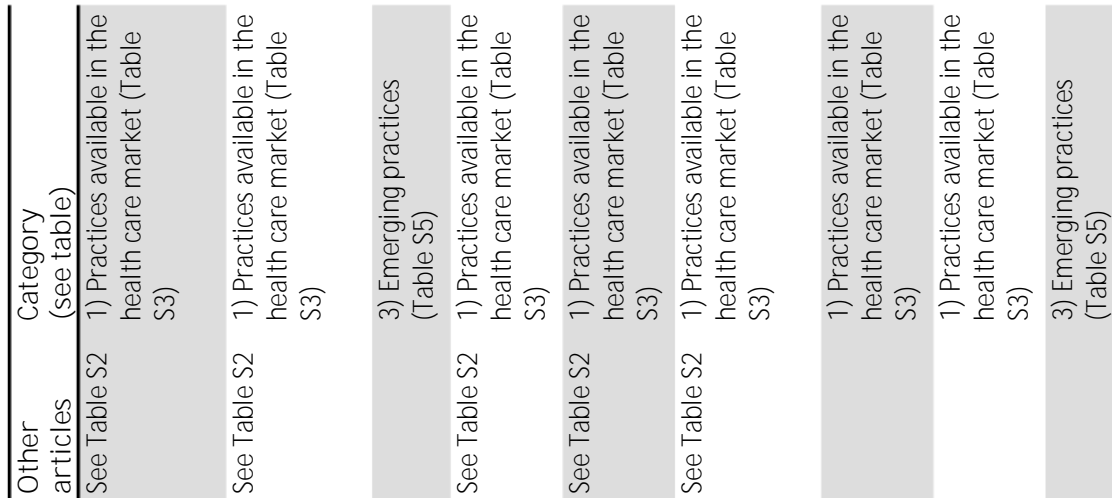

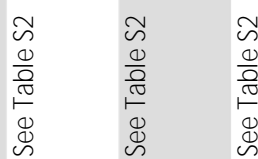

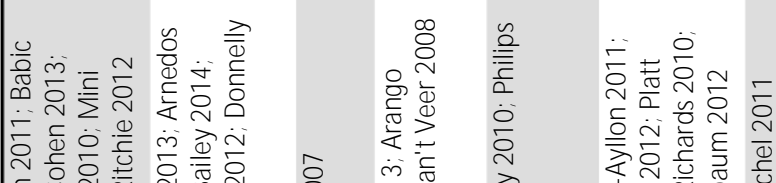

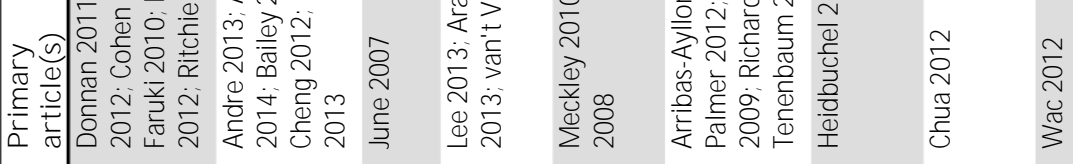

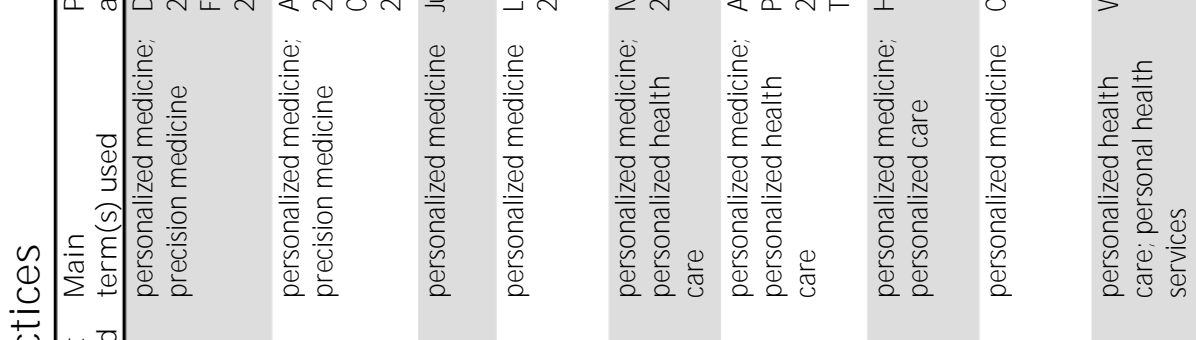

\&ิ छे

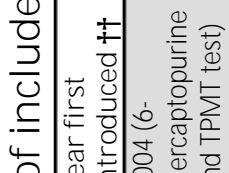

\&্

8

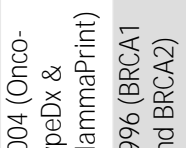

.

$\frac{0}{\frac{0}{0}}$ 

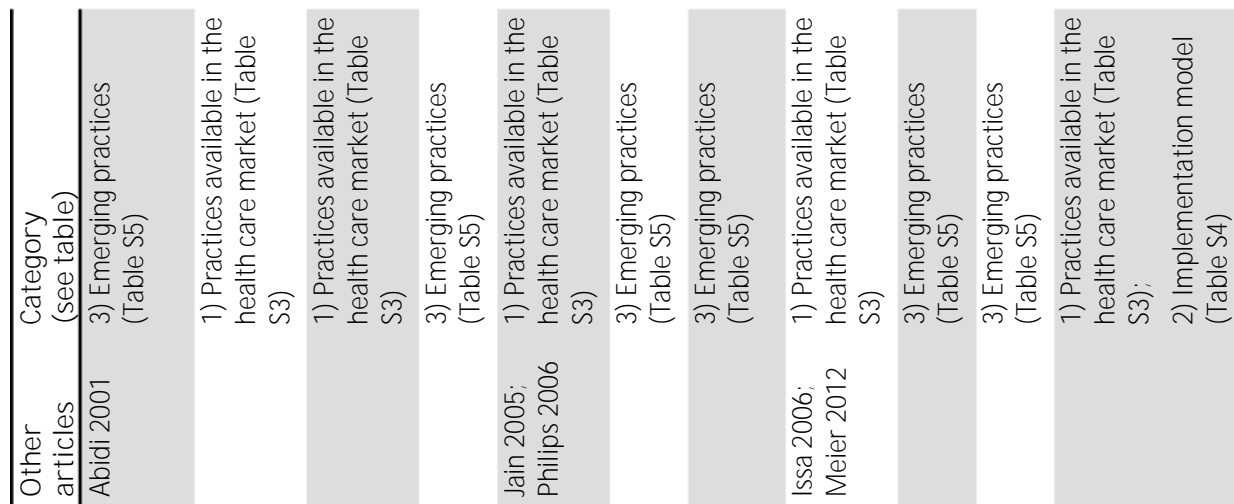

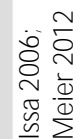

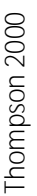

$m$
$\frac{1}{2}$
$\frac{0}{\circ}$
$\frac{1}{\circ}$

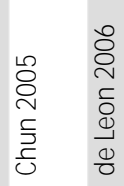

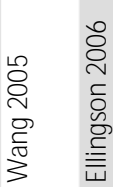

กั

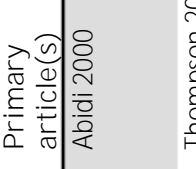

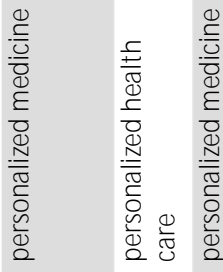

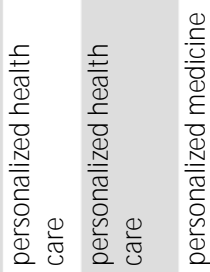

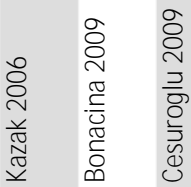

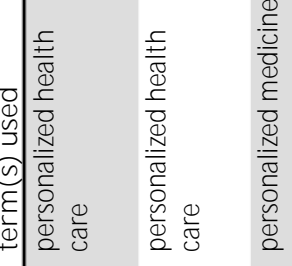

\&

\&ి

\&

:

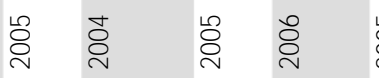
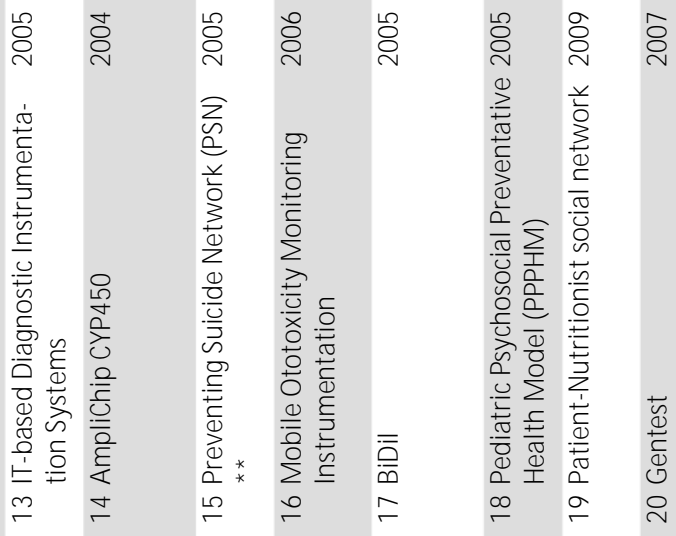

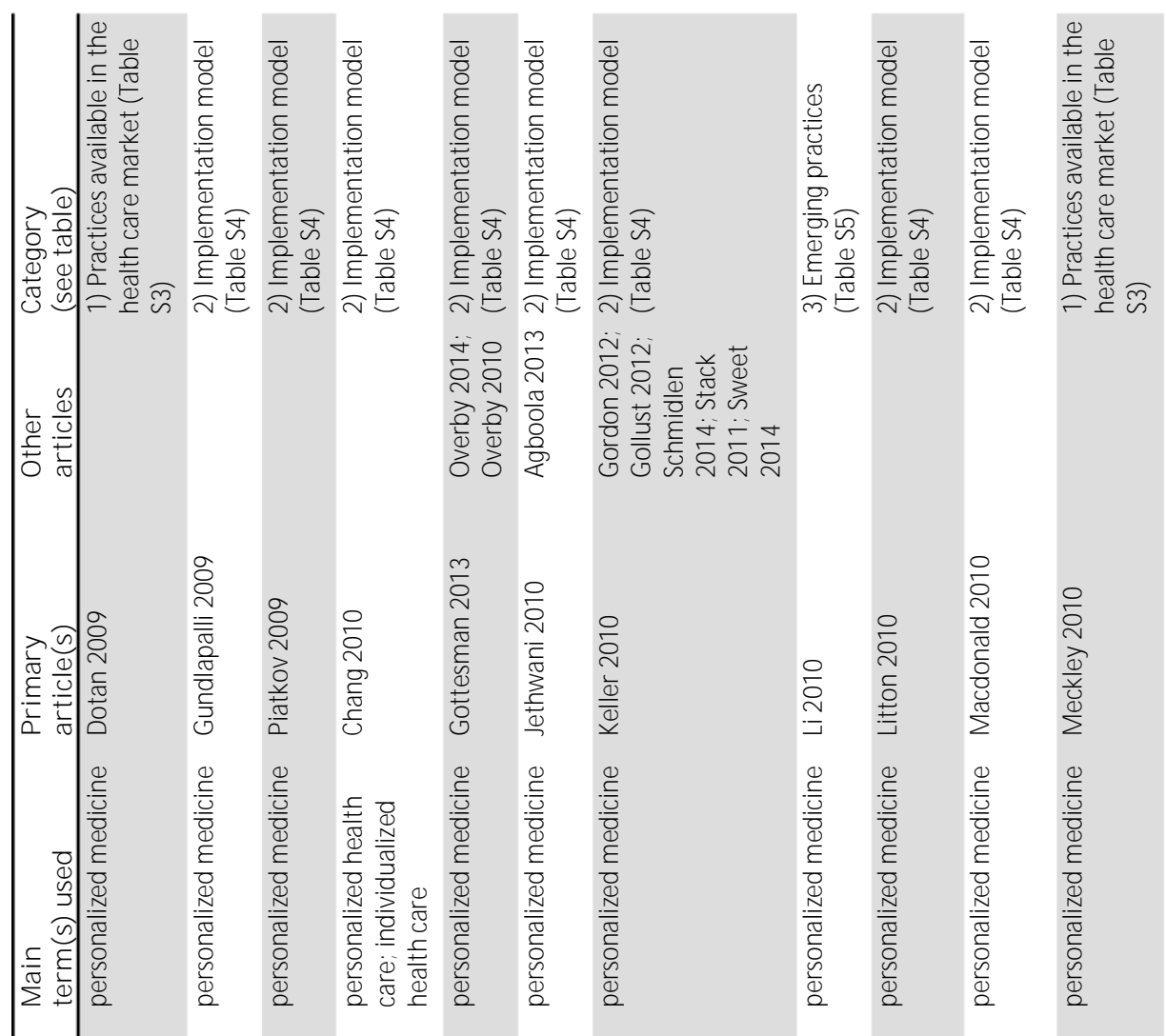

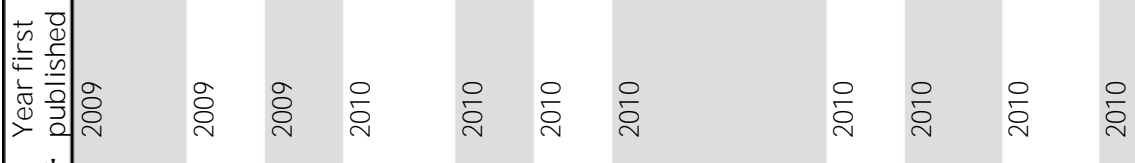

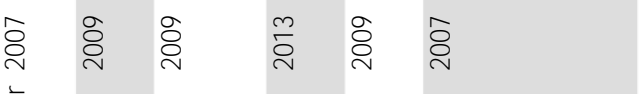

ำ
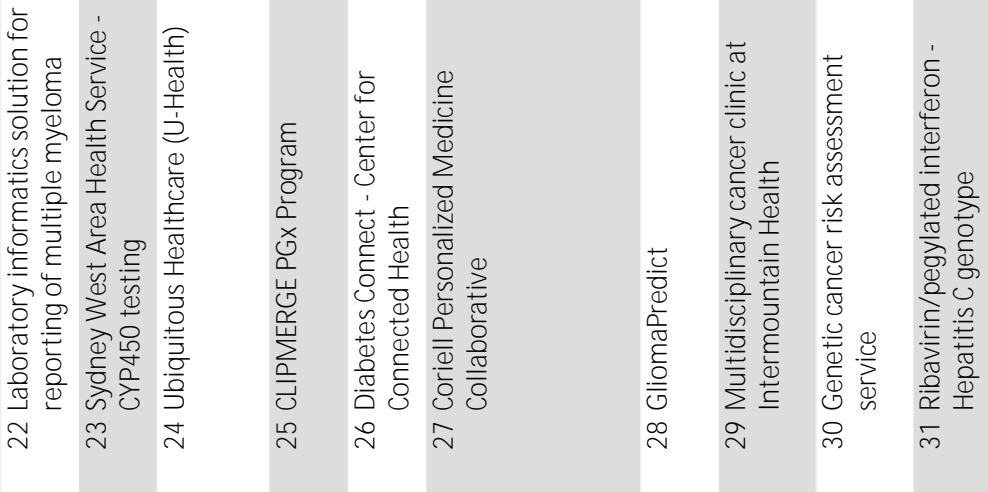


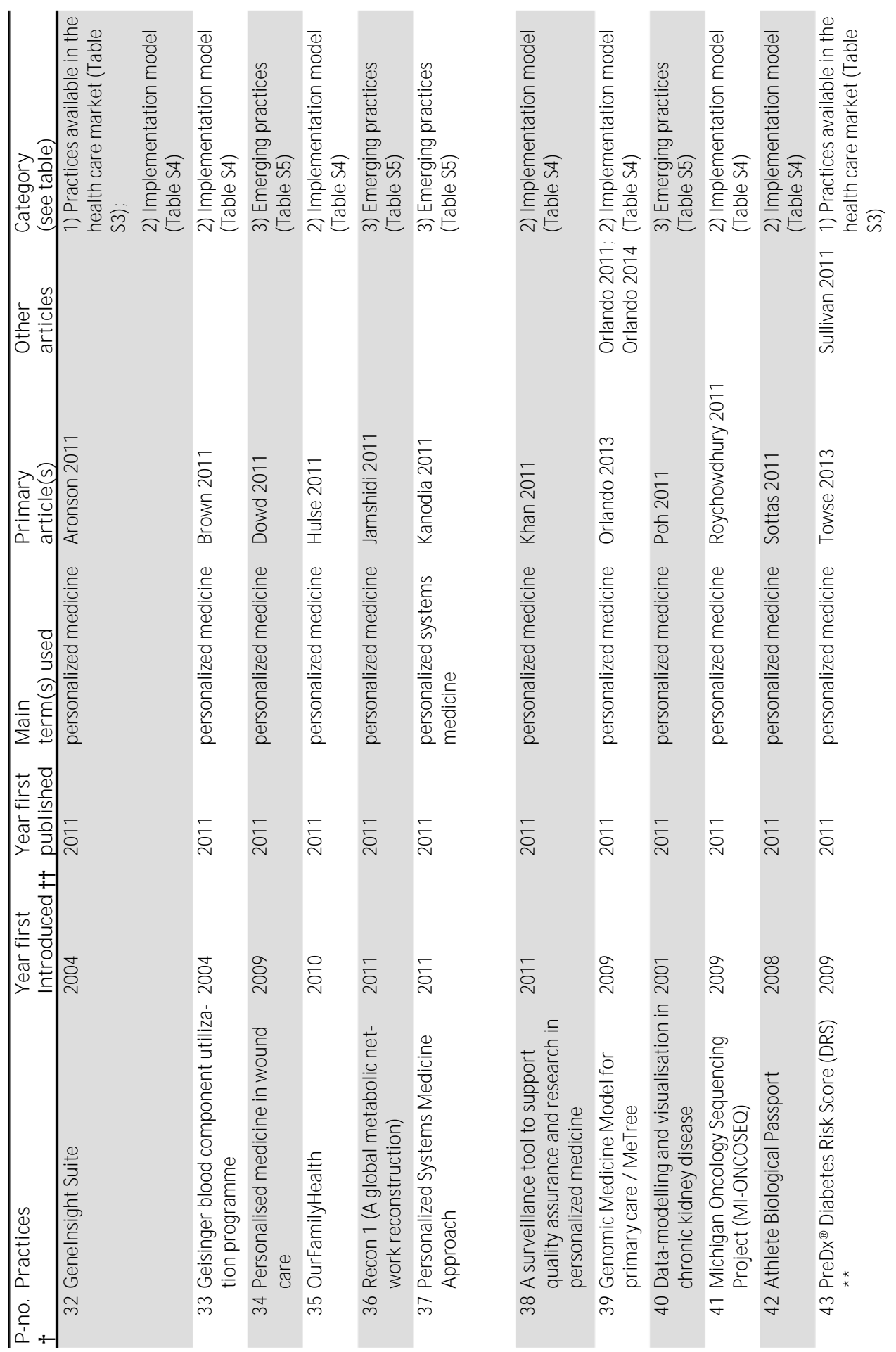




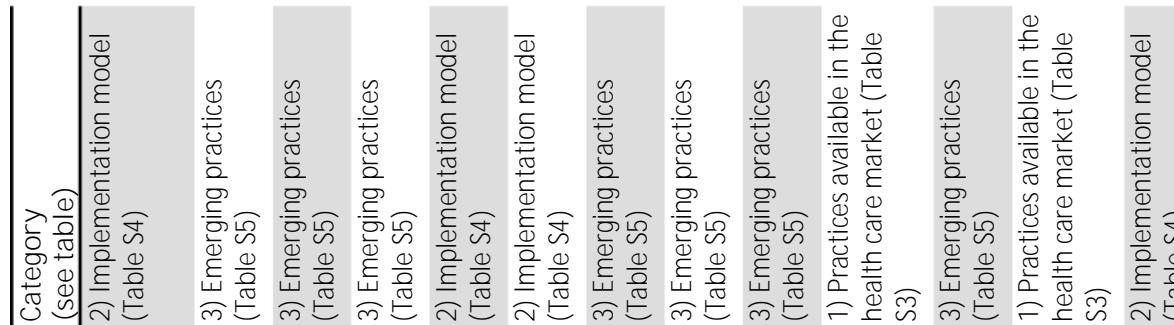

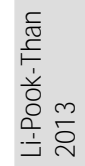

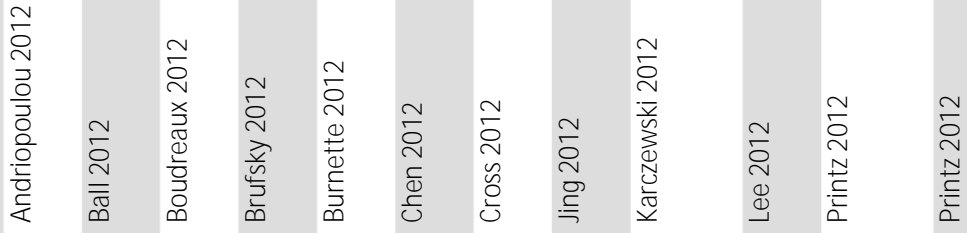

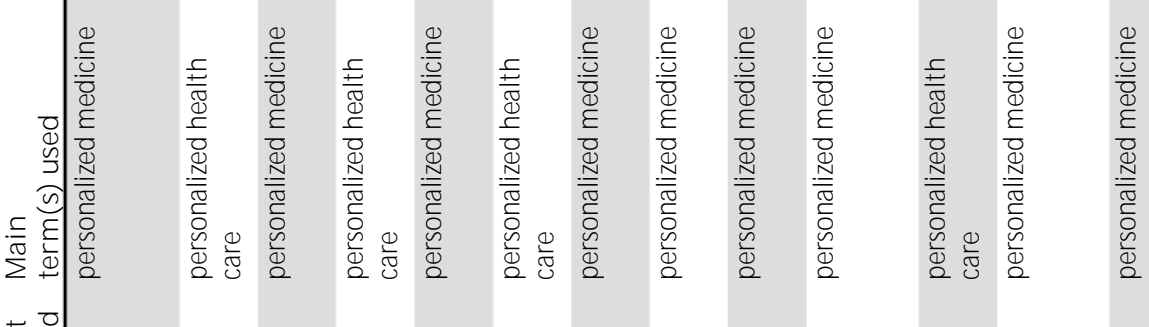

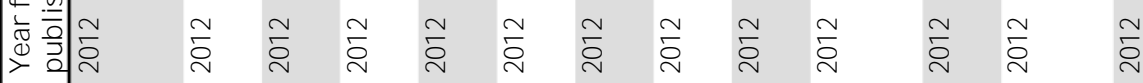

ปี

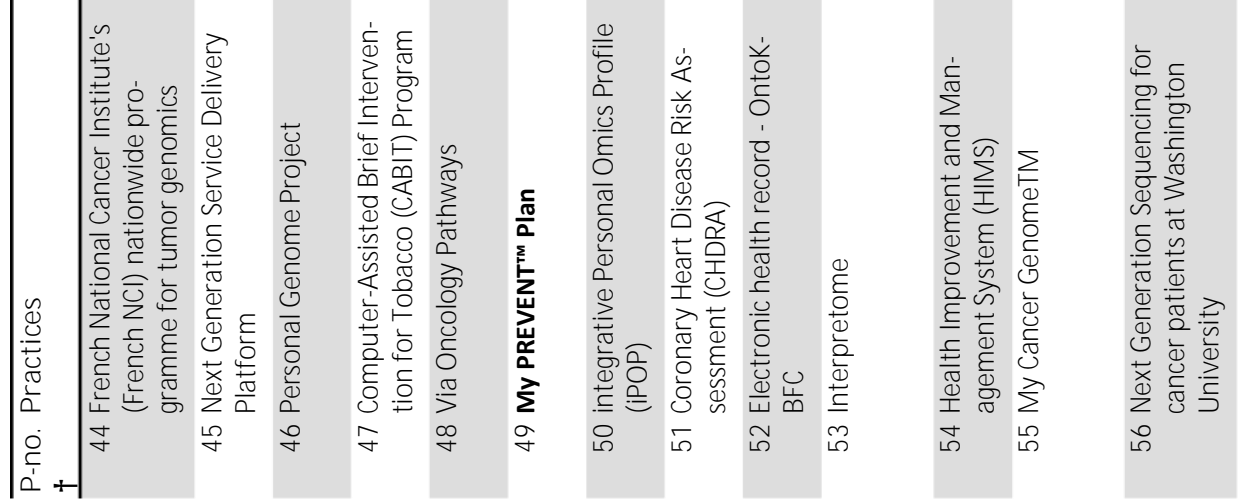




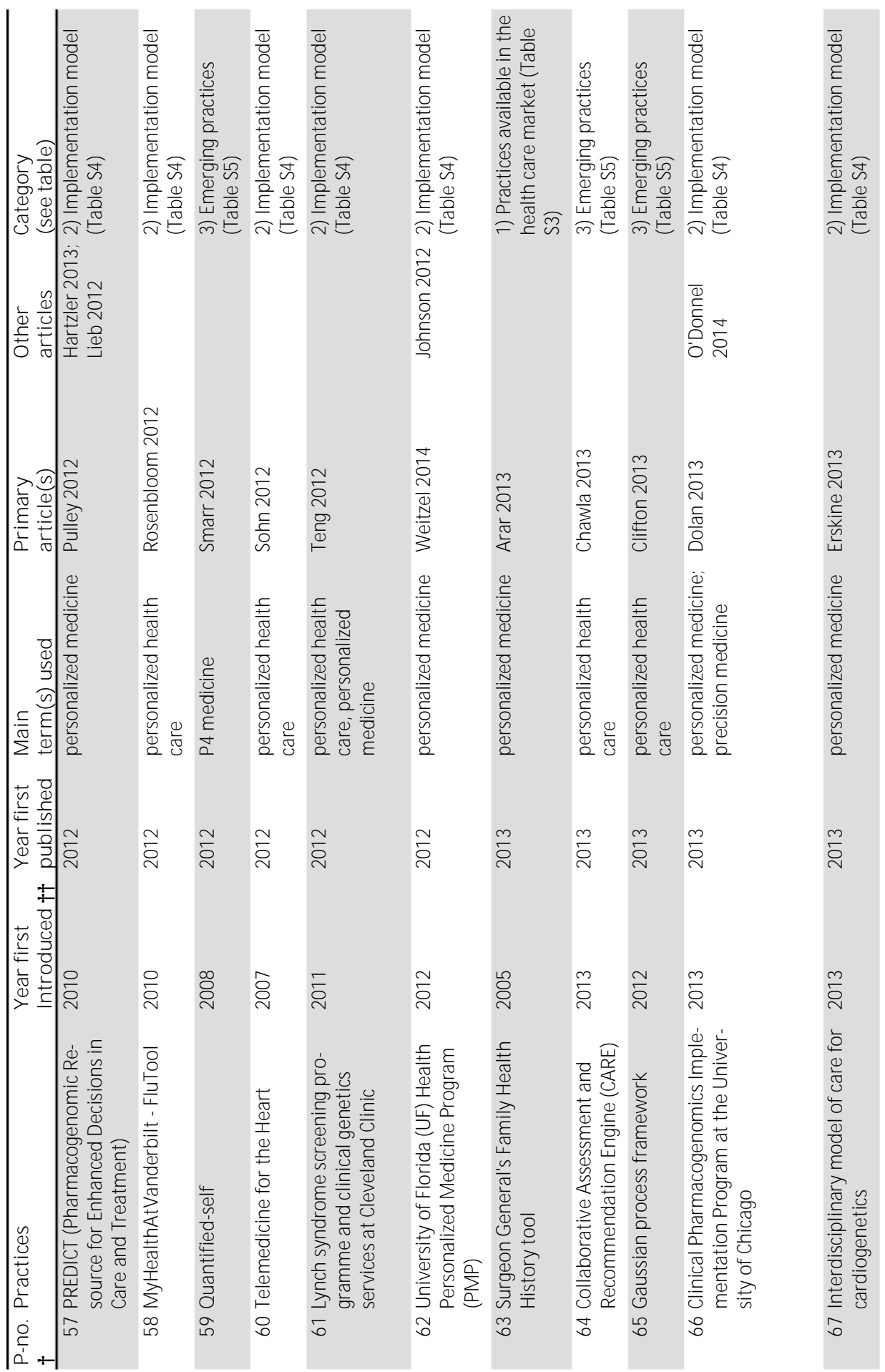




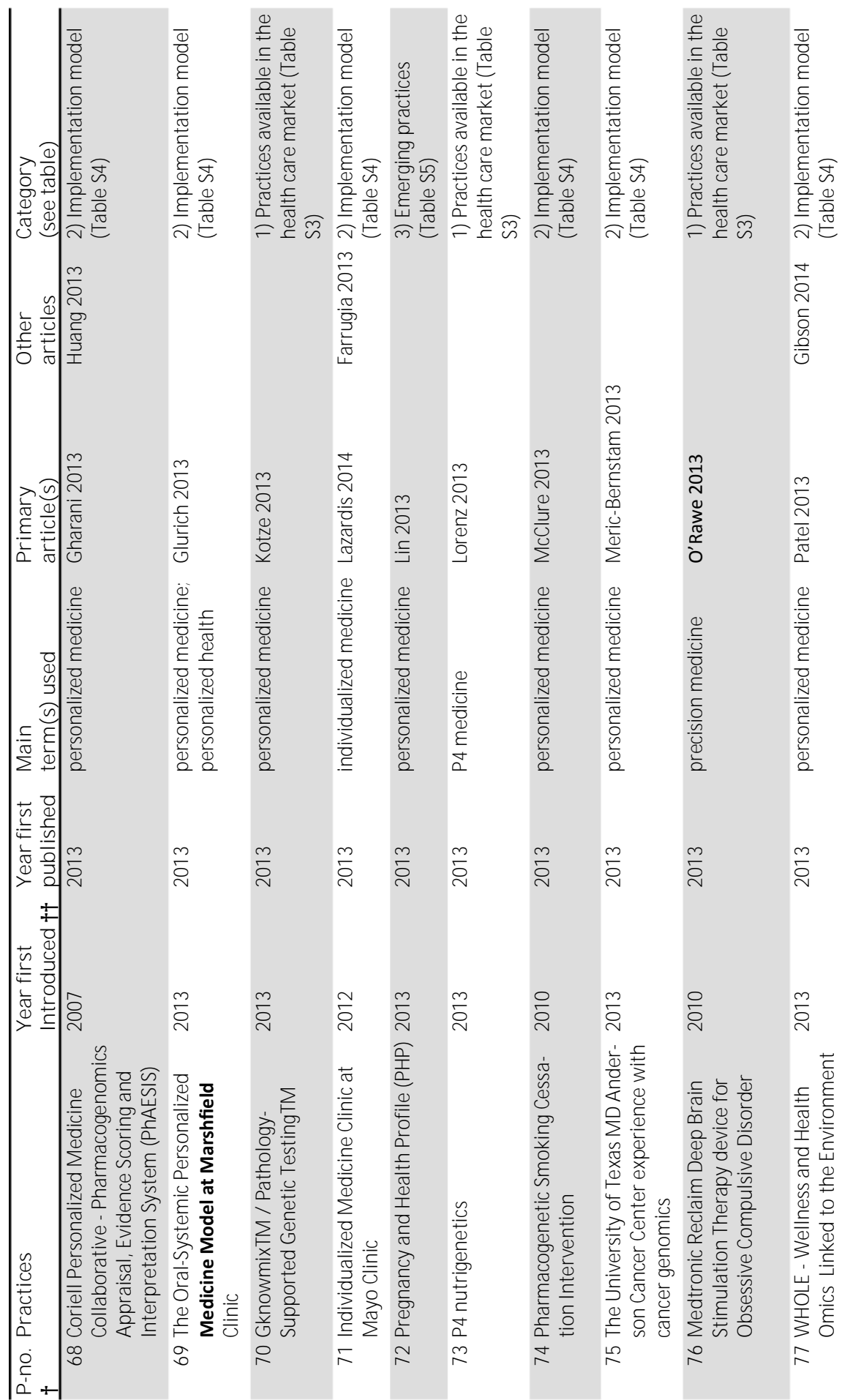




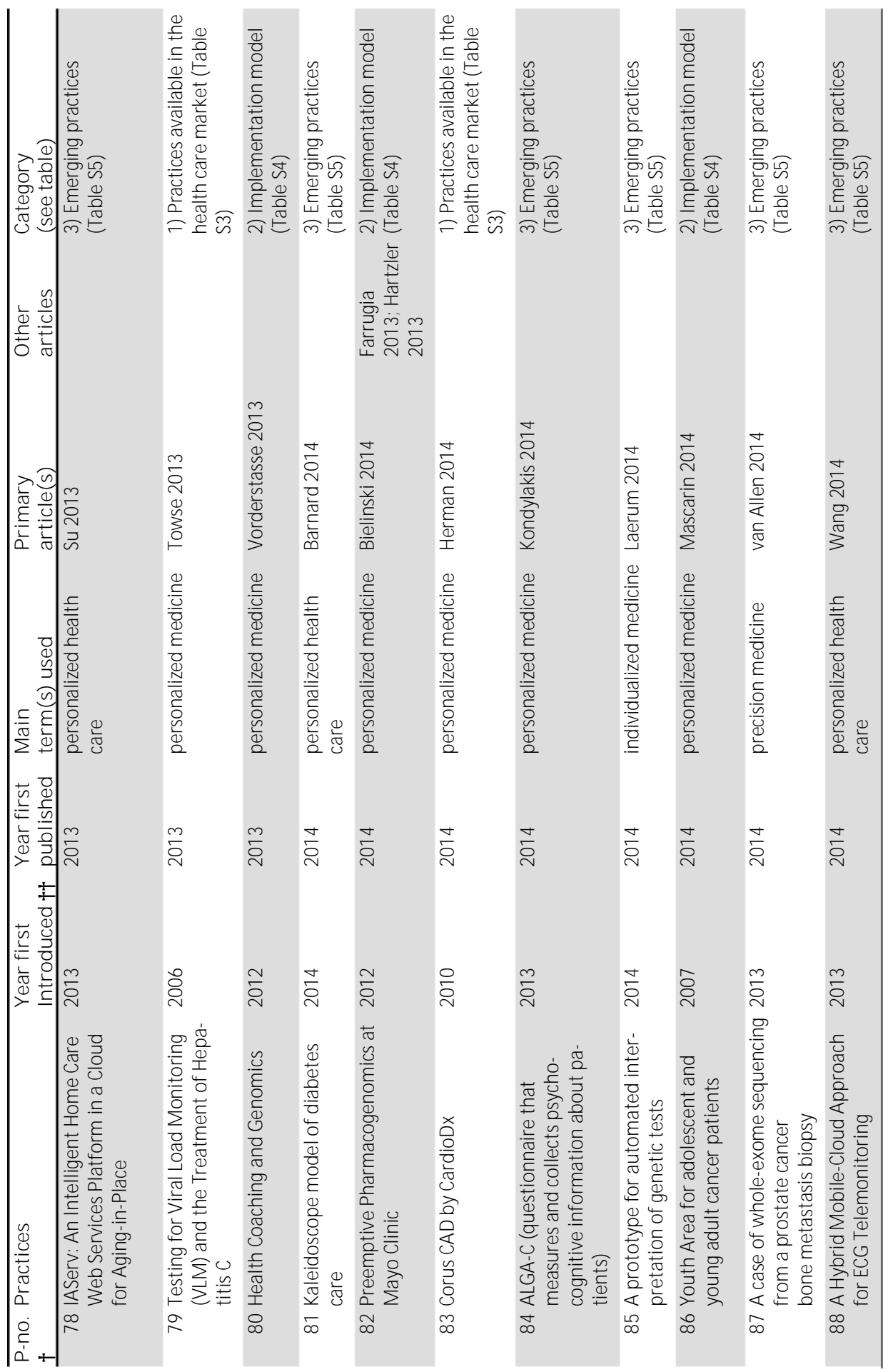




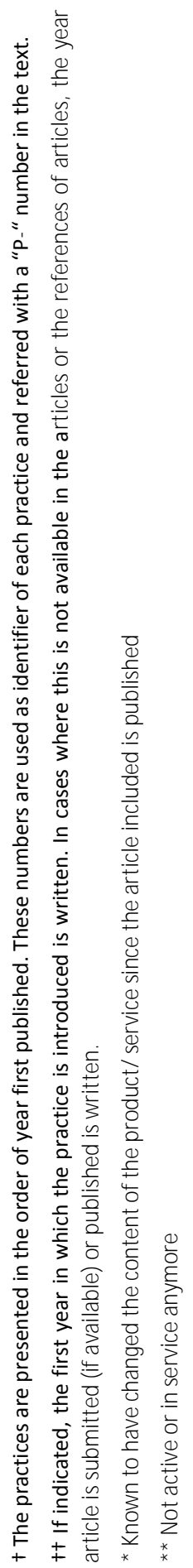




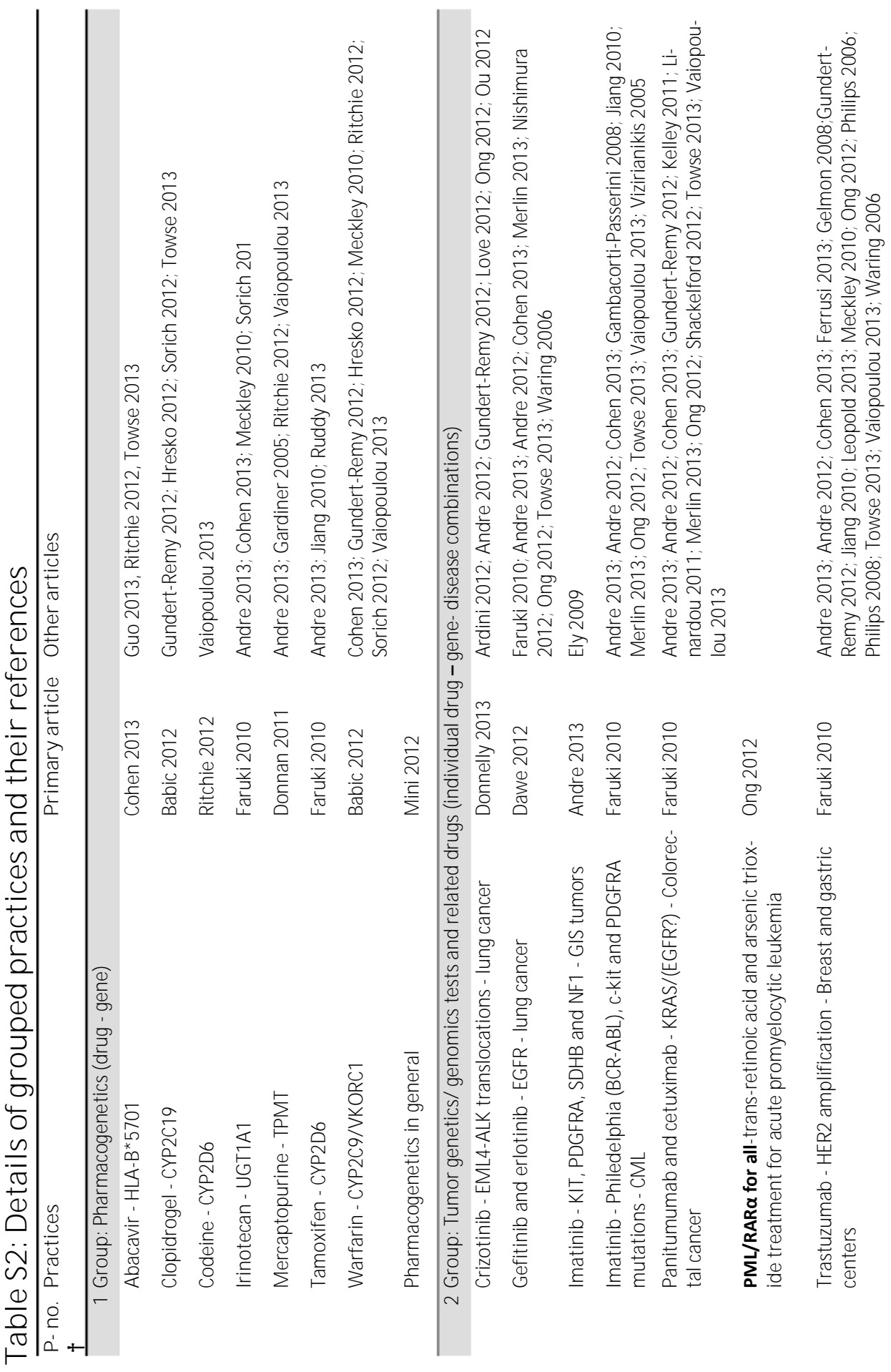




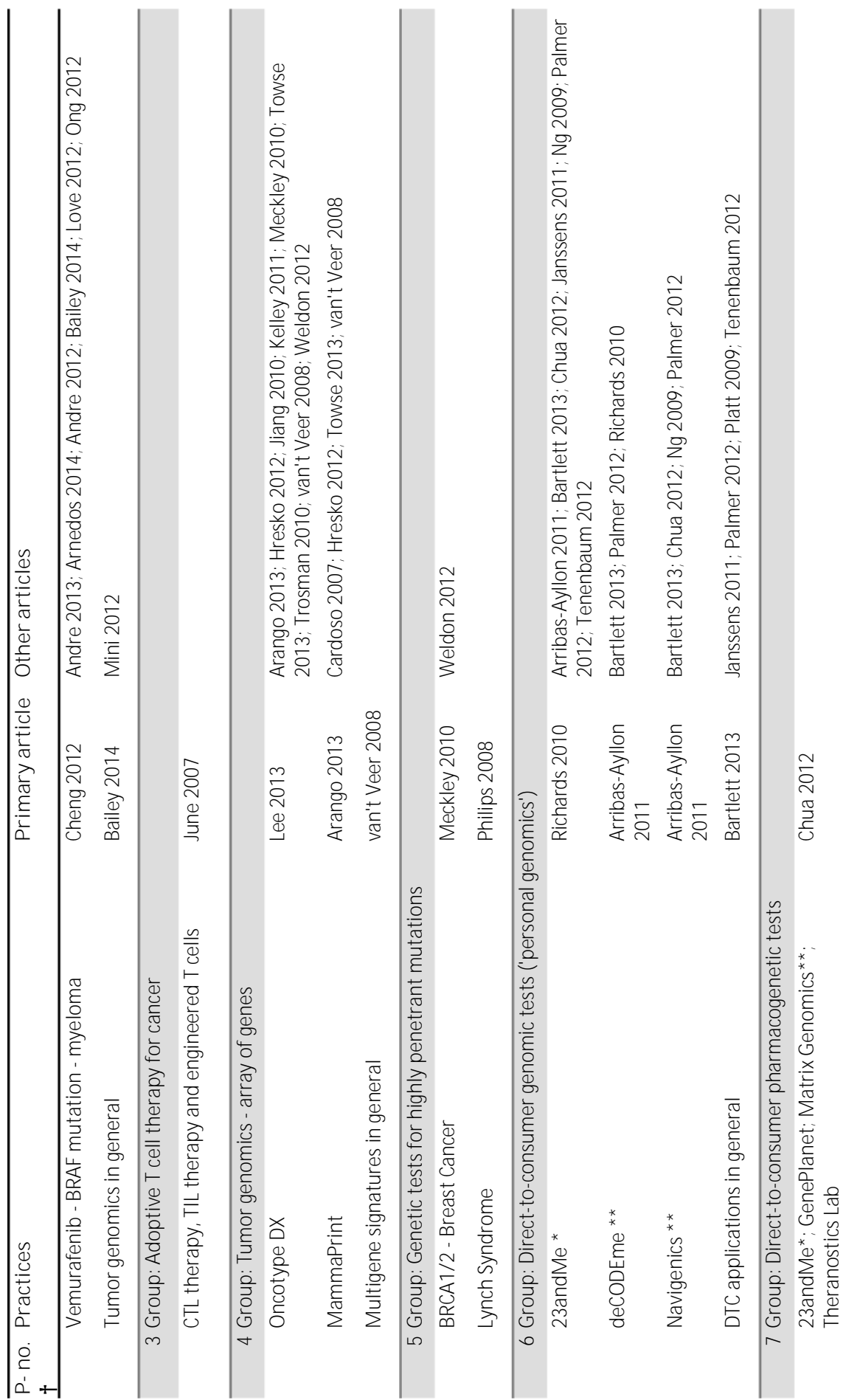




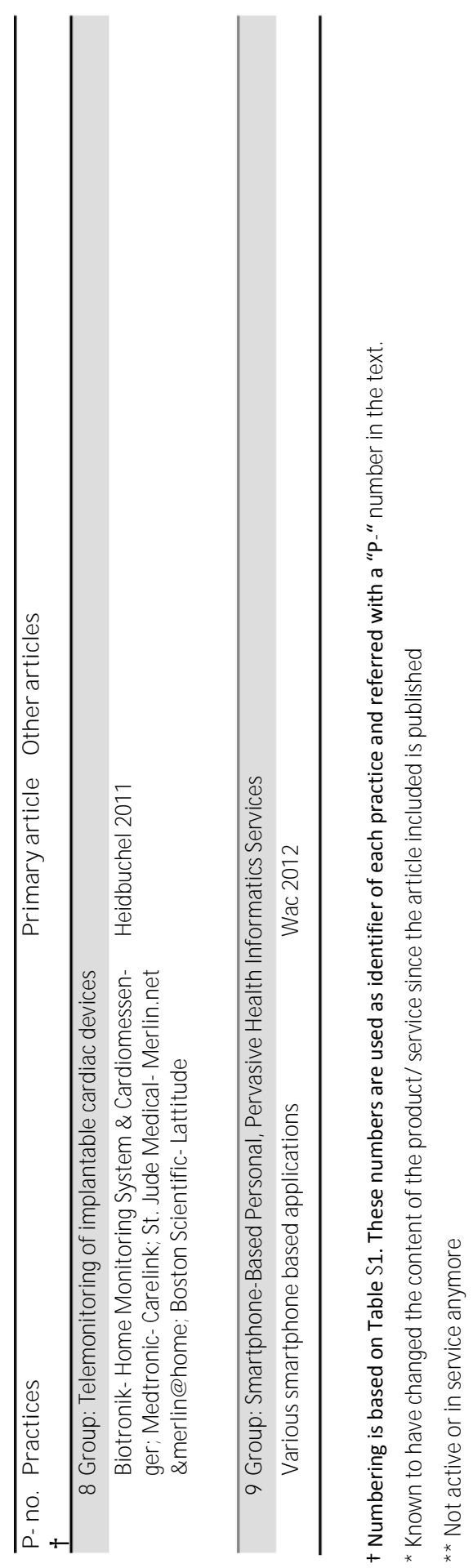




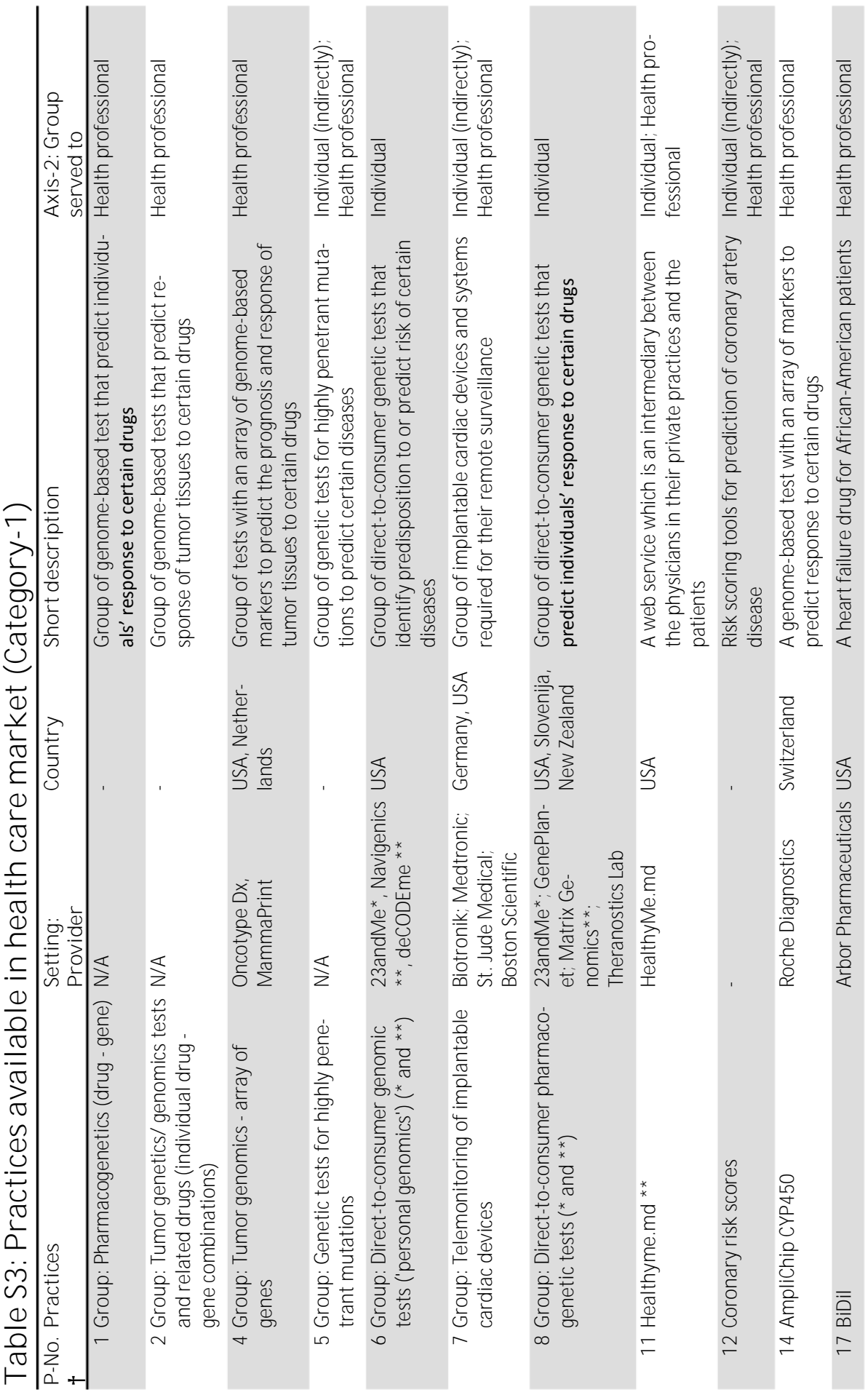




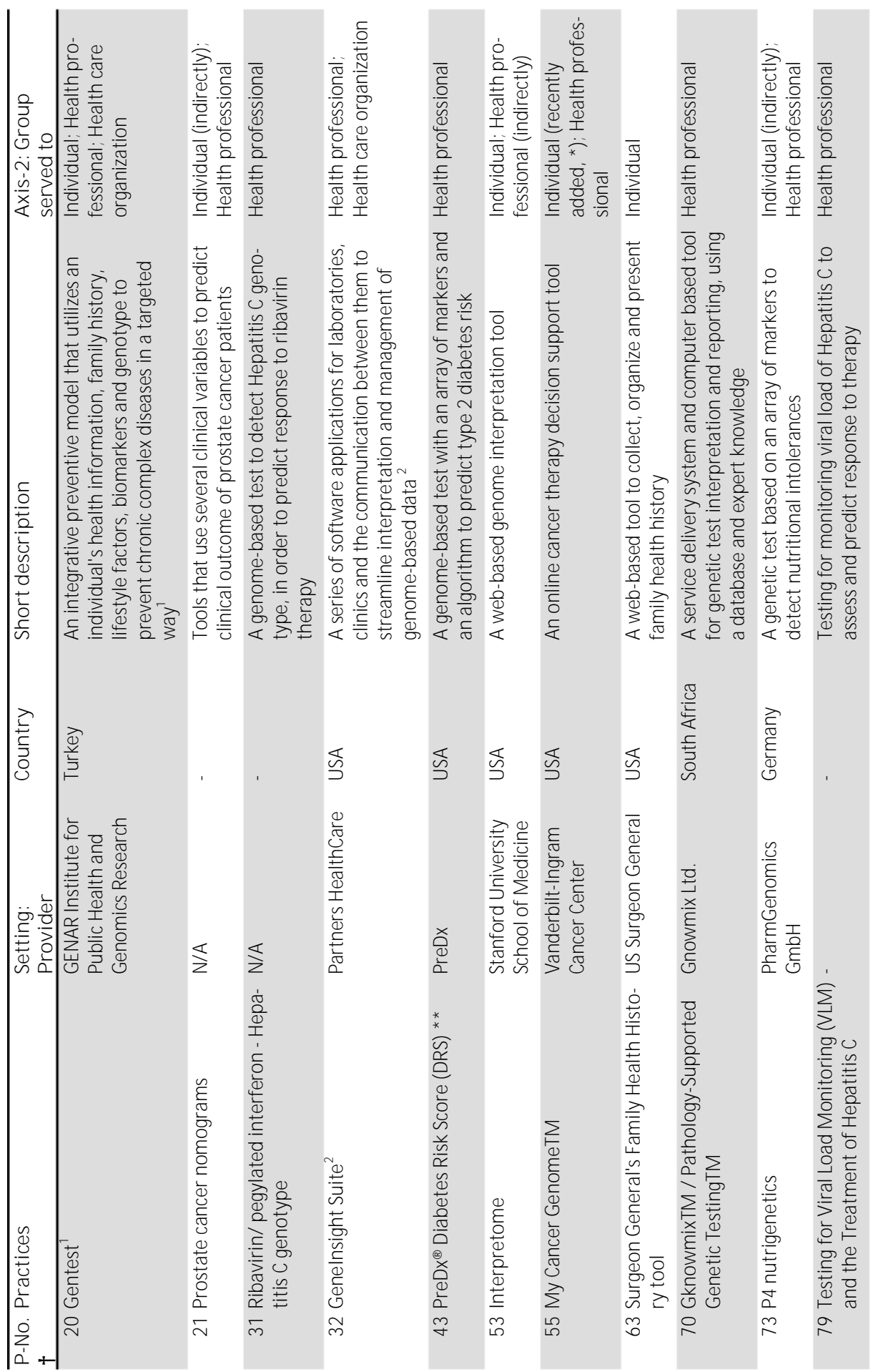




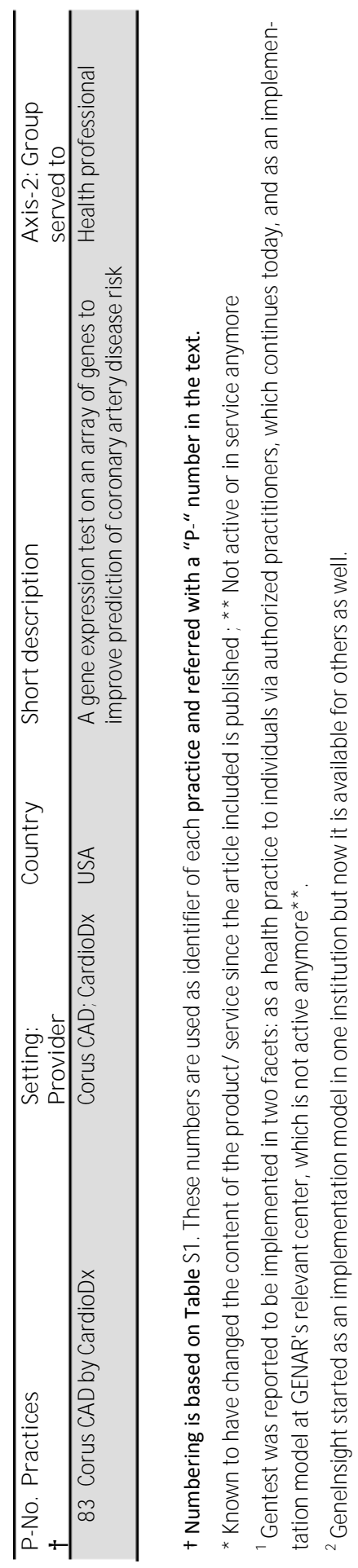




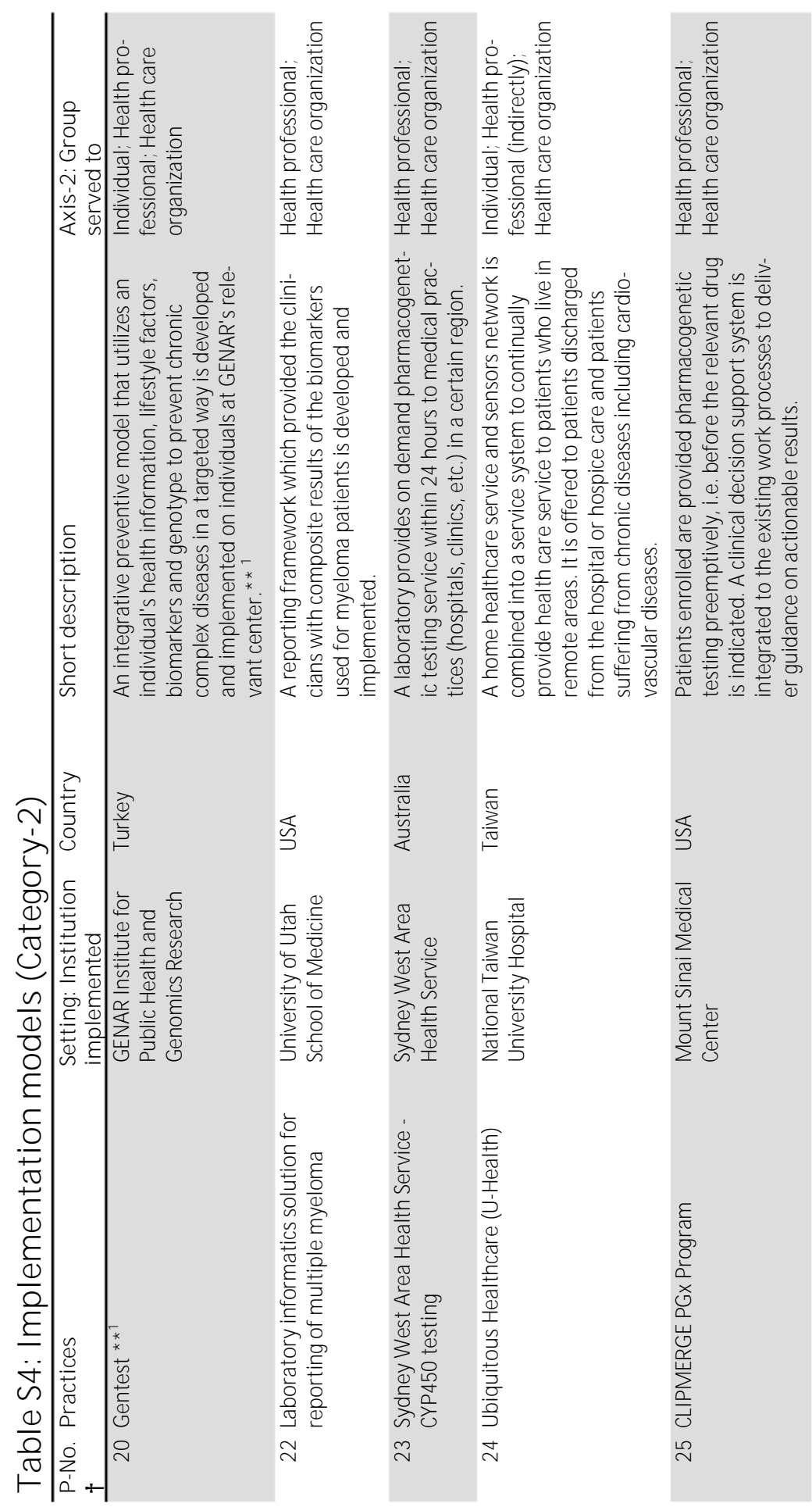




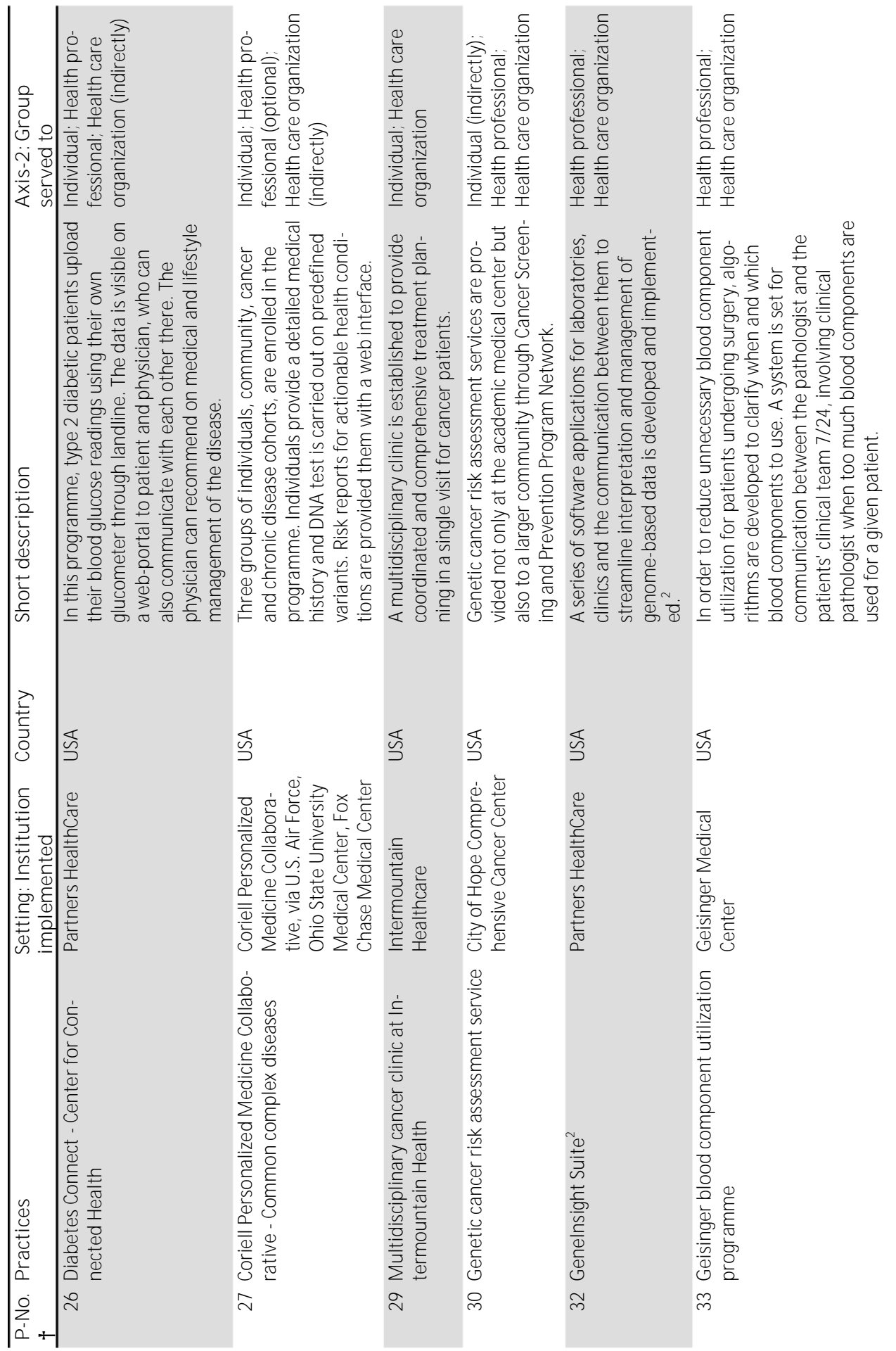




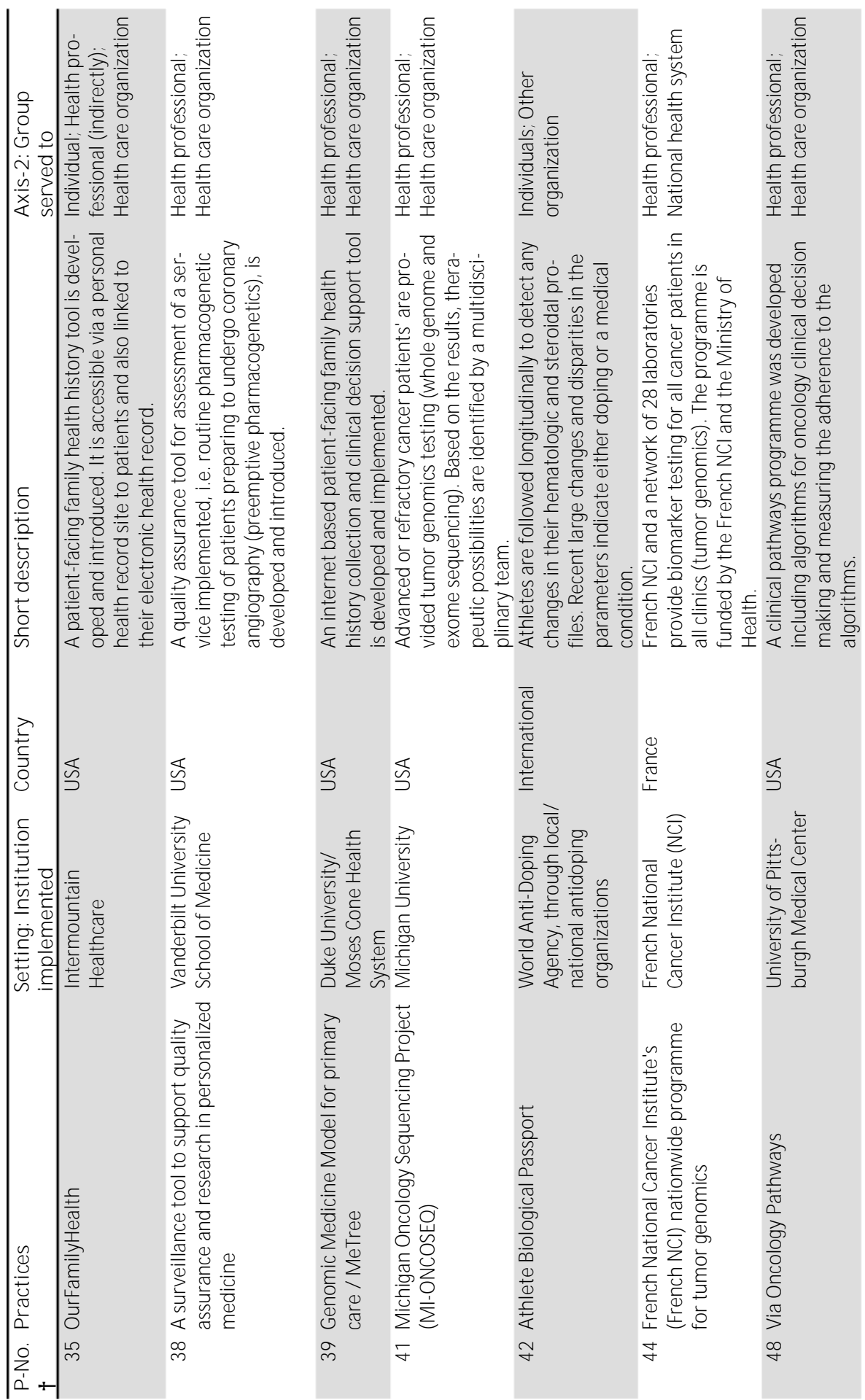




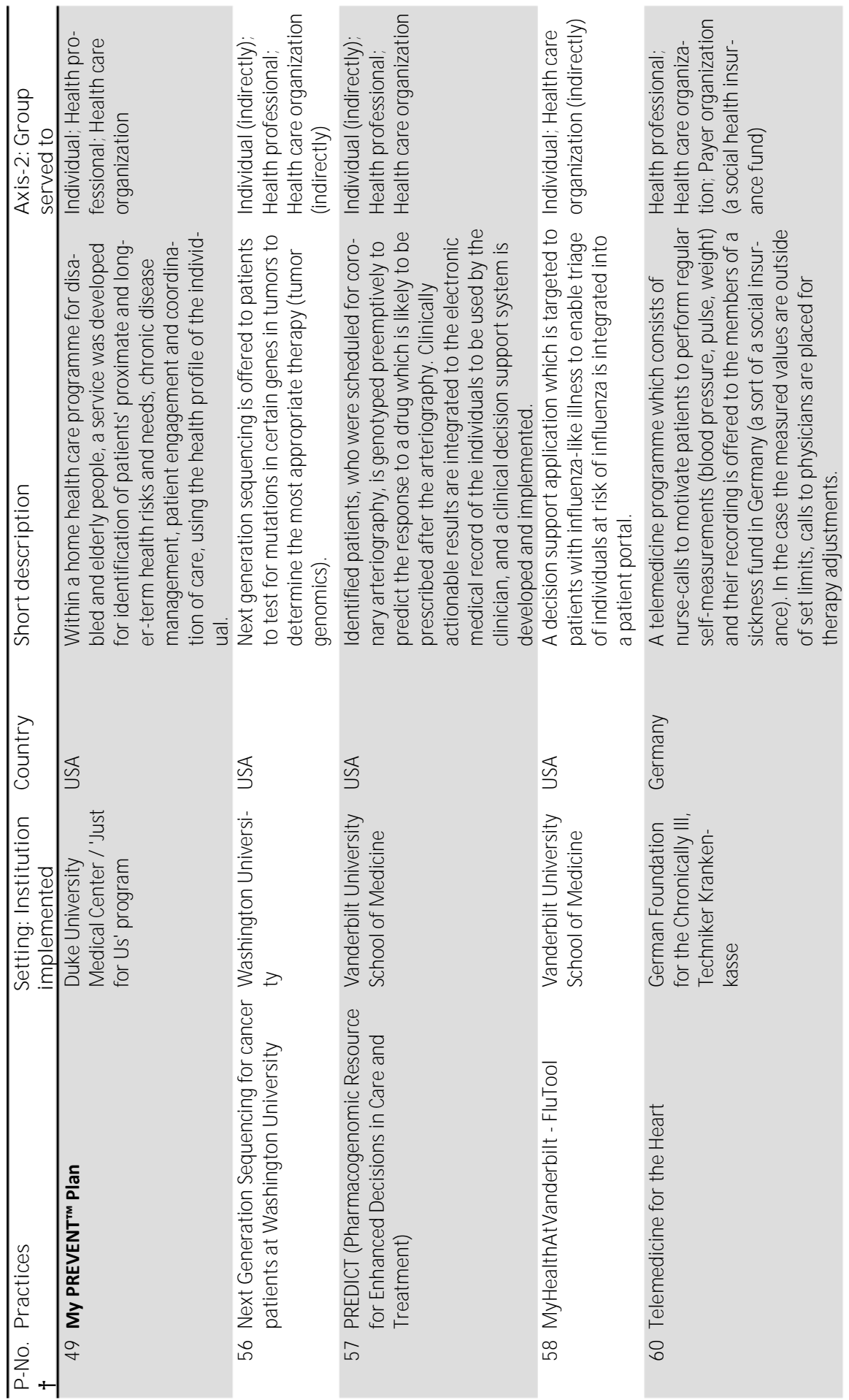




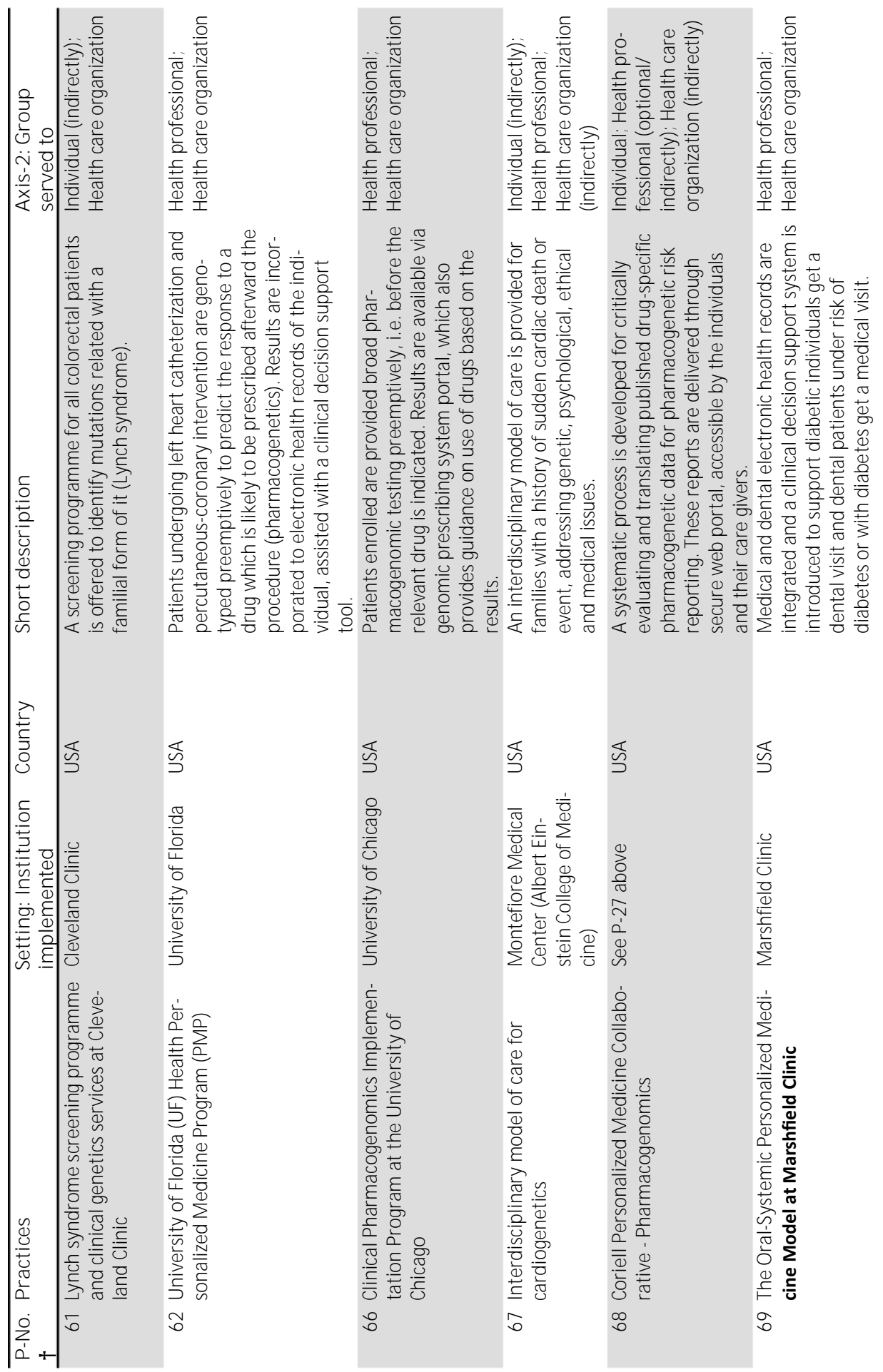




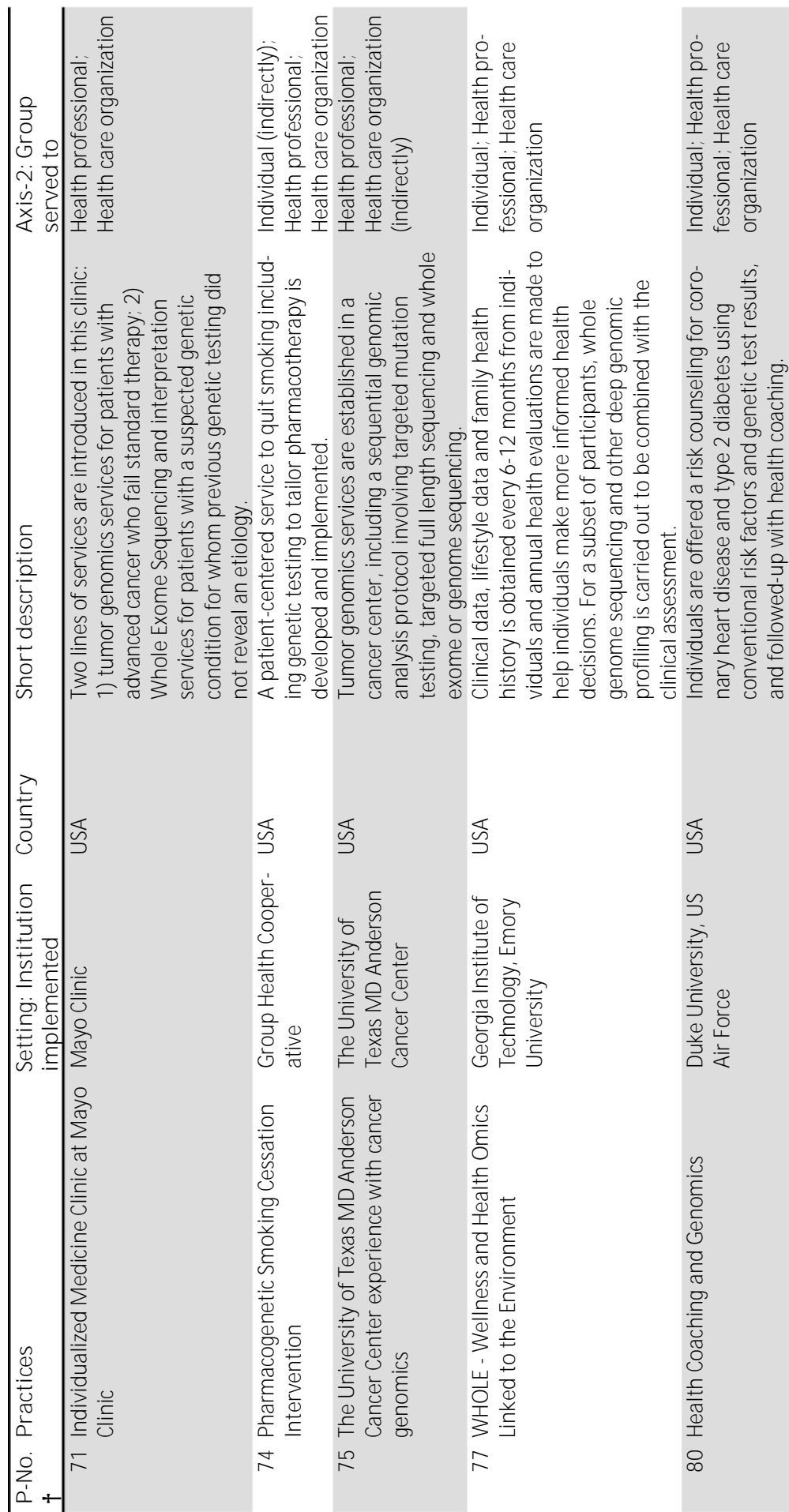




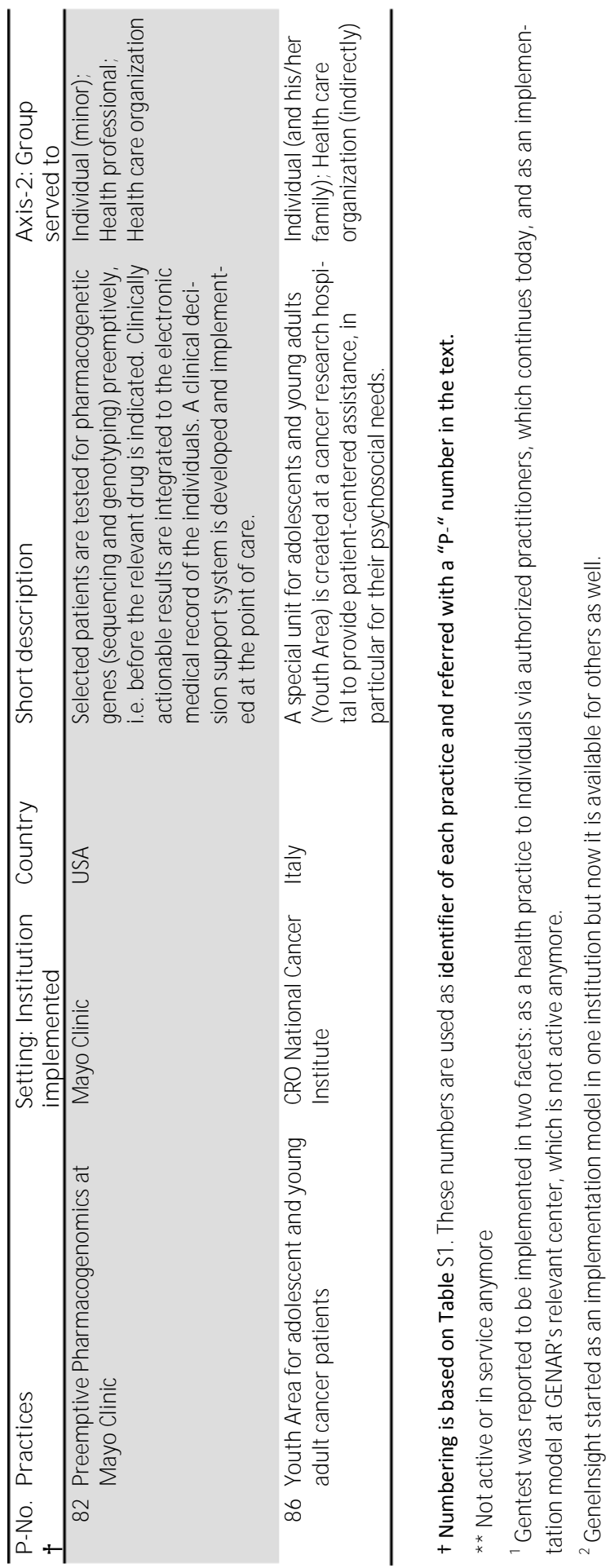




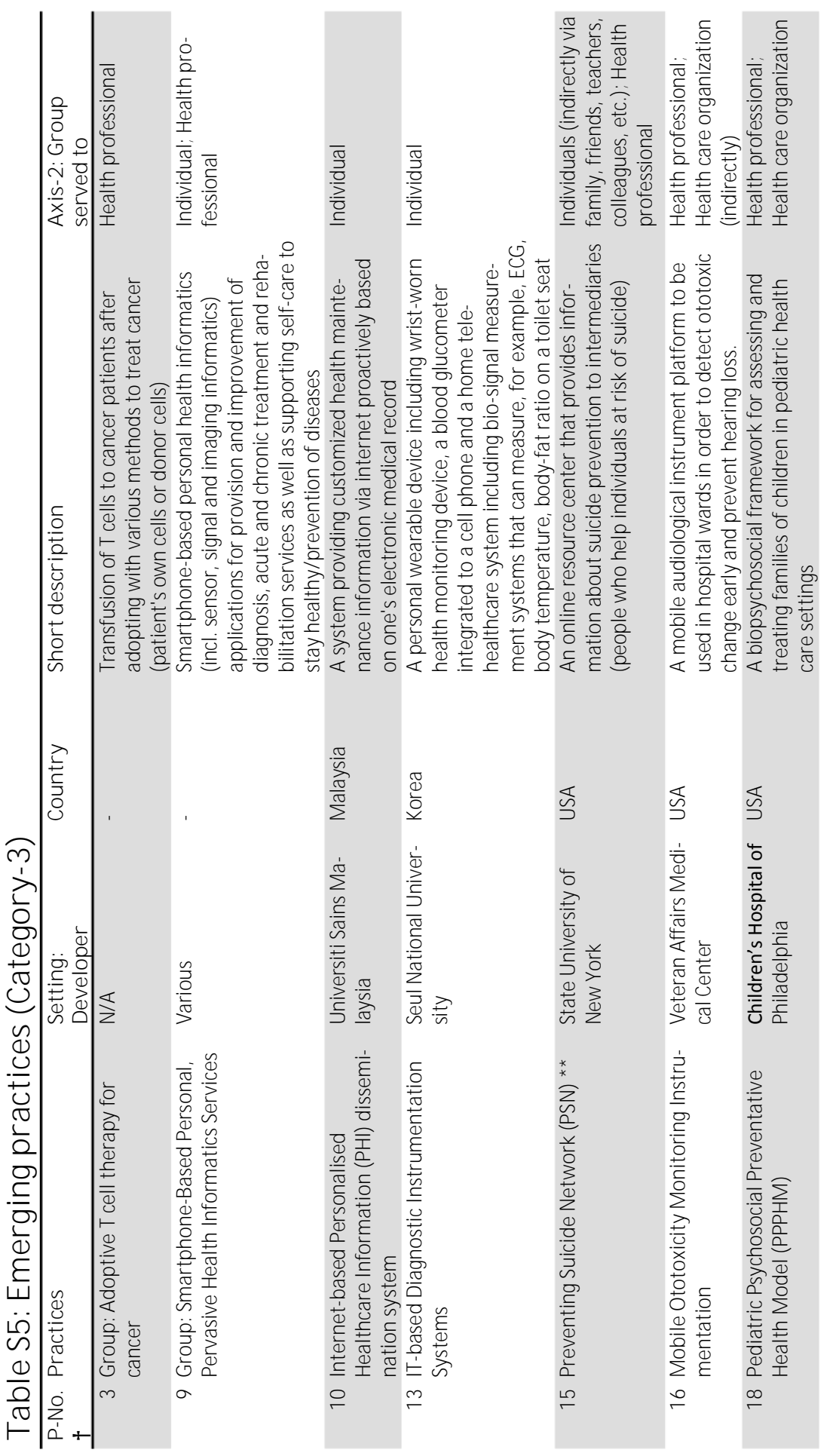




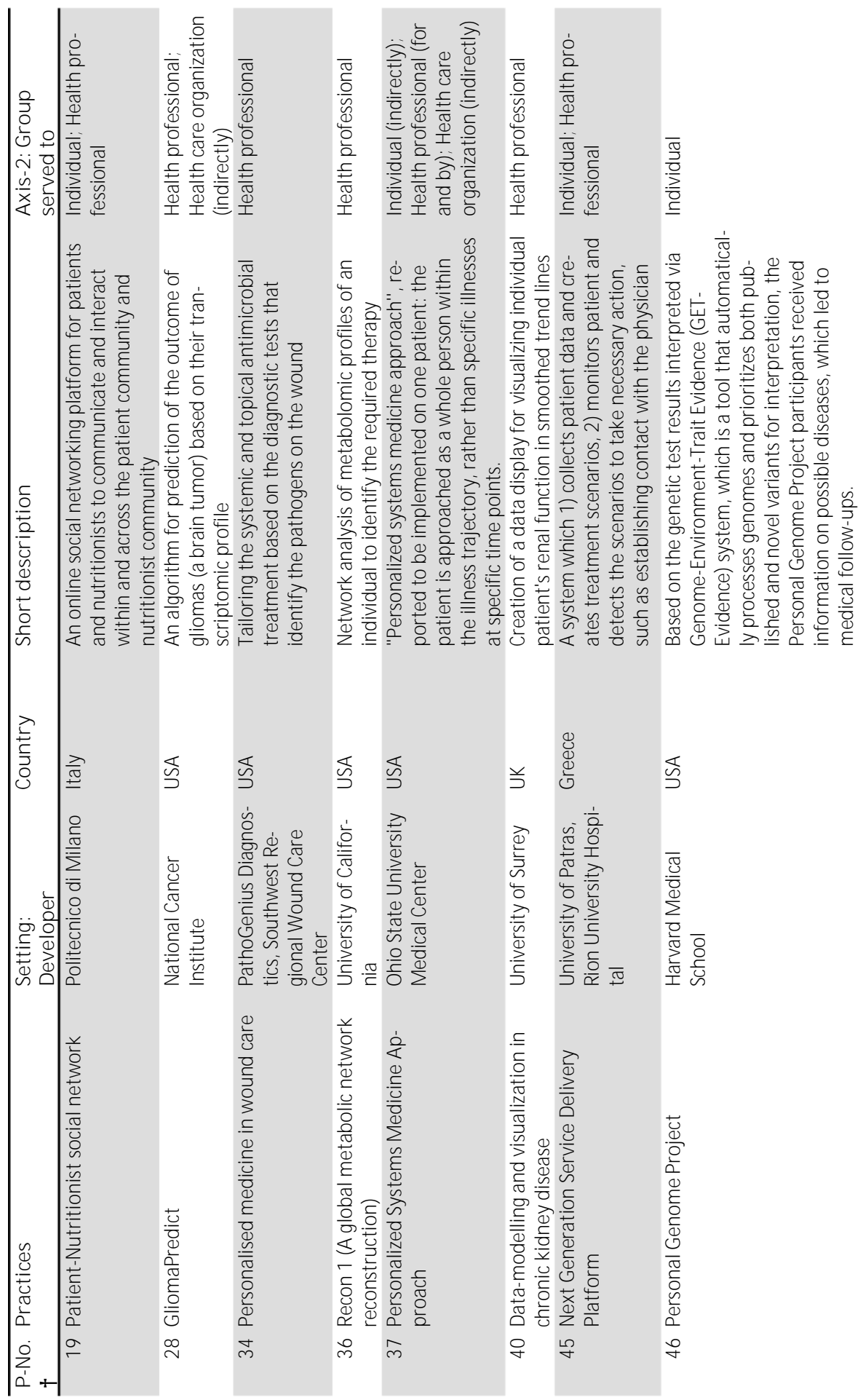




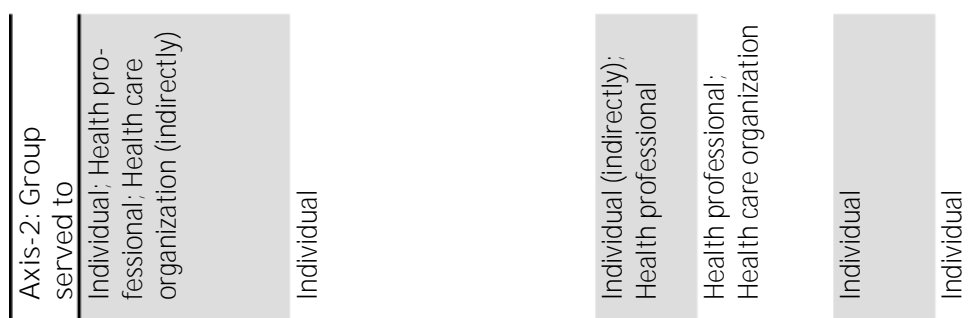

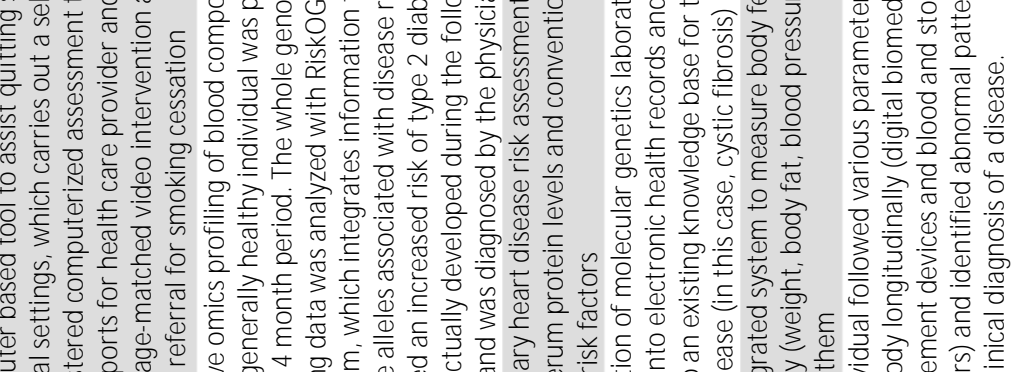

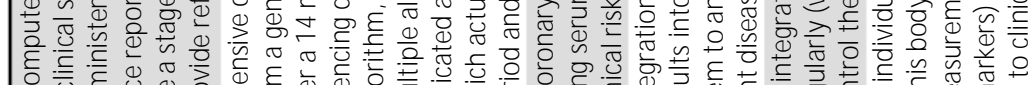

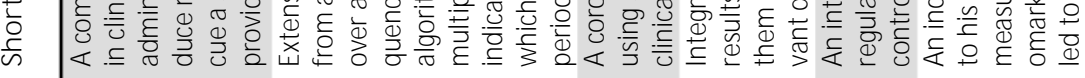

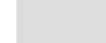




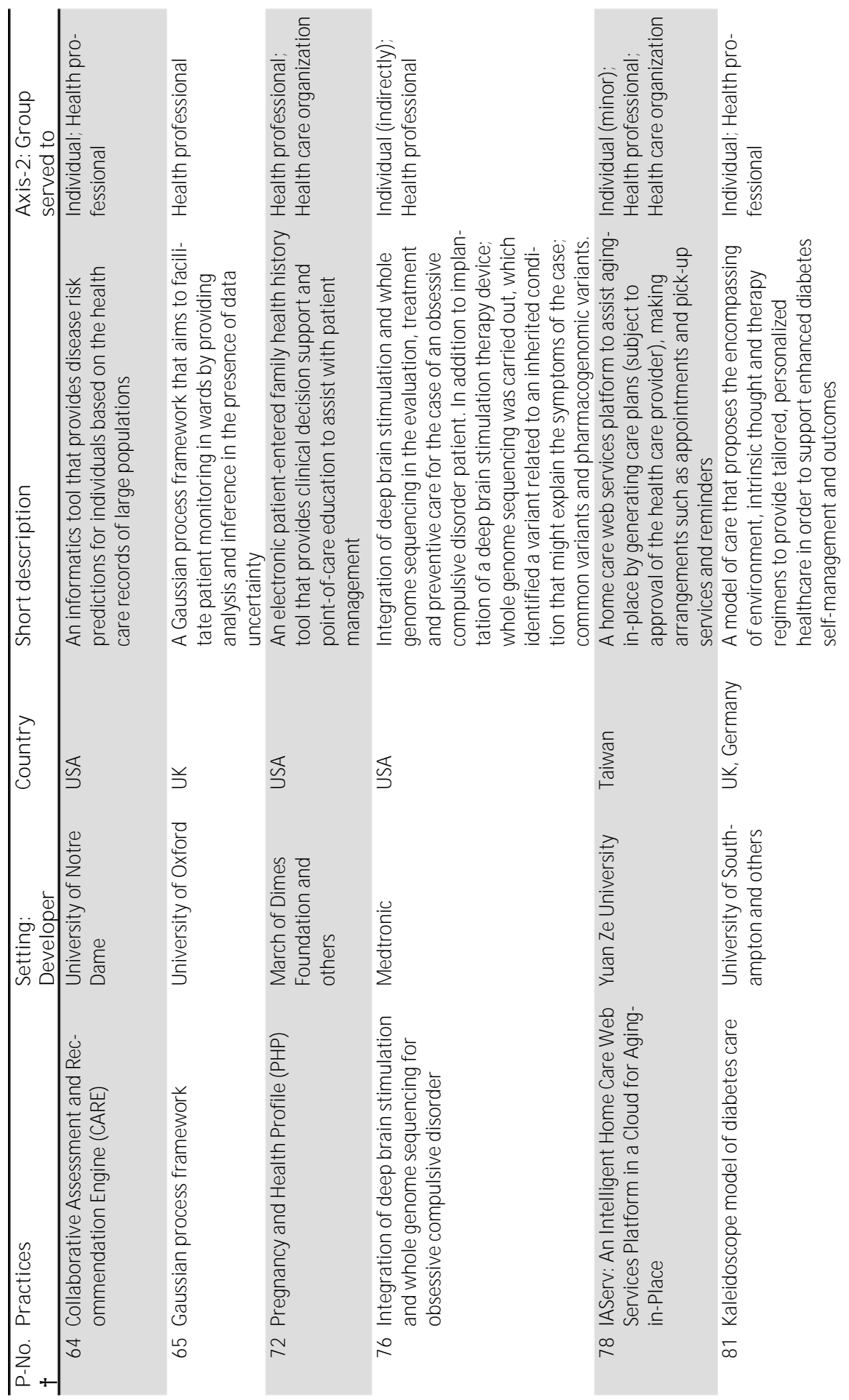




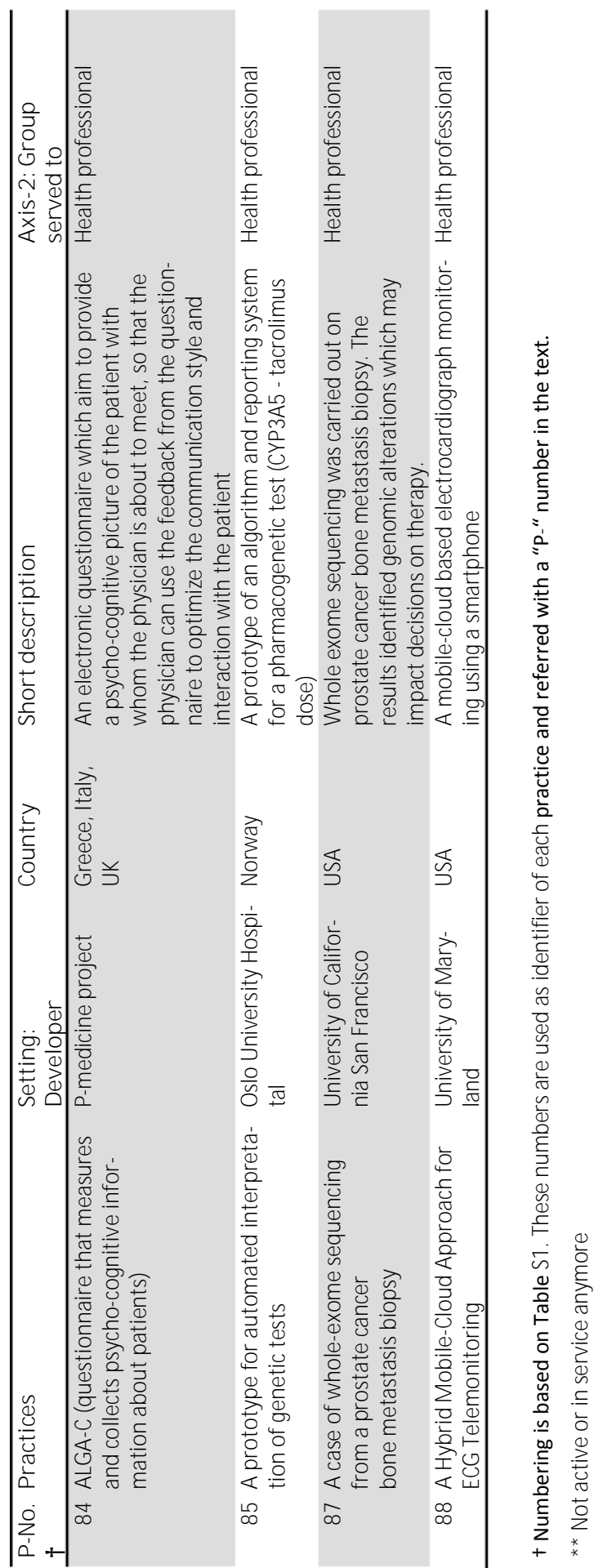




\section{References}

Abidi SS, Goh A. A personalised Healthcare Information Delivery System: pushing customised healthcare information over the WWW. Studies in health technology and informatics. 2000;77:663-7.

Abidi SS, Han CY, Abidi SR. Patient empowerment via 'pushed' delivery of Personalised Healthcare educational content over the internet. Studies in health technology and informatics. 2001;84(Pt 2):1425-9.

Agboola SO, Ball M, Kvedar JC, Jethwani K. The future of Connected Health in preventive medicine. QJM : monthly journal of the Association of Physicians. 2013;106(9):791-4.

Andre F, Ciccolini J, Spano JP, Penault-Llorca F, M ounier N, Freyer G, et al. Personalized medicine in oncology: where have we come from and where are we going? Pharmacogenomics. 2013;14(8):931-9.

Andre F, Nowak F, Arnedos M, Lacroix L, Viens P, Calvo F. Biomarker discovery, development, and implementation in France: a report from the French National Cancer Institute and cooperative groups. Clinical cancer research : an official journal of the American Association for Cancer Research. 2012;18(6):1555-60.

Andriopoulou FG, Lazarou NG, Lymberopoulos DK. A proposed Next Generation Service Delivery Platform (NG-SDP) for eHealth domain. Conference proceedings : Annual International Conference of the IEEE Engineering in Medicine and Biology Society IEEE Engineering in Medicine and Biology Society Conference. 2012;2012:6064-7.

Arango BA, Rivera CL, Gluck S. Gene expression profiling in breast cancer. American journal of translational research. 2013;5(2):132-8.

Arar N, Seo J, Abboud HE, Parchman M, Noel P. Veterans' experience in using the online Surgeon General's family health history tool. Personalized medicine. 2011;8(5):523-32.

Ardini E, Galvani A. ALK Inhibitors, a Pharmaceutical Perspective. Frontiers in oncology. 2012;2:17.

Arnedos M, Vielh P, Soria JC, Andre F. The genetic complexity of common cancers and the promise of personalized medicine: is there any hope? The Journal of pathology. 2014;232(2):274-82.

Aronson SJ, Clark EH, Babb LJ, Baxter S, Farwell LM, Funke BH, et al. The Genelnsight Suite: a platform to support laboratory and provider use of DNA-based genetic testing. Human mutation. 2011;32(5):532-6.

Arribas-Ayllon M, Sarangi S, Clarke A. Promissory accounts of personalisation in the commercialisation of genomic knowledge. Communication \& medicine. 2011;8(1):53-66.

Babic N. Clinical pharmacogenomics and concept of personalized medicine. Journal of Medical Biochemistry. 2012;31(4):281-6.

Bailey AM, Mao Y, Zeng J, Holla V, Johnson A, Brusco L, et al. Implementation of biomarker-driven cancer therapy: existing tools and remaining gaps. Discovery medicine. 2014;17(92):101-14.

Ball MP, Thakuria JV, Zaranek AW, Clegg T, Rosenbaum AM, Wu X, et al. A public resource facilitating clinical use of genomes. Proceedings of the National Academy of Sciences of the United States of America. 2012;109(30):11920-7.

Barnard KD, Lloyd CE, Dyson PA, Davies MJ, O'Neil S, Kamunilli N, et al. Kaleidoscope model of diabetes care: time for a rethink? Diabetic medicine : a journal of the British Diabetic Association. 2014.

Bartlett G, Avard D, Knoppers BM. A new twist on an old problem: Primary care physicians and results from direct-to-consumer genetic testing. Personalized M edicine. 2013;10(8):827-33. 
Bielinski SJ, Olson JE, Pathak J, Weinshilboum RM, Wang L, Lyke KJ, et al. Preemptive genotyping for personalized medicine: design of the right drug, right dose, right time-using genomic data to individualize treatment protocol. M ayo Clinic proceedings. 2014;89(1):25-33.

Bonacina S, Privitera YA, M arsilio S, M ontin E, Passarelli F, Masseroli M, et al. A web-based tool for cooperating behaviors in eating and physical activity control. Studies in health technology and informatics. 2009;144:155-9.

Boudreaux ED, Bedek KL, Byrne NJ, Baumann BM , Lord SA, Grissom G. The Computer-Assisted Brief Intervention for Tobacco (CABIT) program: a pilot study. Journal of medical Internet research. 2012;14(6):e163.

Brown RE, Dorion RP, Trowbridge C, Stammers AH, Fitt W, Davis J. Algorithmic and consultative integration of transfusion medicine and coagulation: a personalized medicine approach with reduced blood component utilization. Annals of clinical and laboratory science. 2011;41(3):211-6.

Brufsky A, Lokay K, M cDonald M. Driving Evidence-Based Standardization of Care within a Framework of Personalized Medicine. American Society of Clinical Oncology educational book / ASCO American Society of Clinical Oncology M eeting. 2012;32:e62-e5.

Burnette R, Simmons LA, Snyderman R. Personalized health care as a pathway for the adoption of genomic medicine. Journal of Personalized Medicine. 2012;2(4):232-40.

Cardoso F, Piccart-Gebhart M, Van't Veer L, Rutgers E. The M INDACT trial: the first prospective clinical validation of a genomic tool. M olecular oncology. 2007;1(3):246-51.

Cesuroglu T, Karaca S, Erge S. A practice model for personalized healthcare with a public health genomics perspective. Personalized Medicine. 2009;6(5):567-77.

Chang HL, Shaw MJ, Lai F, Ko WJ, Ho YL, Chen HS, et al. U-Health: An example of a high-quality individualized healthcare service. Personalized M edicine. 2010;7(6):677-87.

Chawla NV, Davis DA. Bringing big data to personalized healthcare: a patient-centered framework. Journal of general internal medicine. 2013;28 Suppl 3:S660-5.

Chen R, Mias GI, Li-Pook-Than J, Jiang L, Lam HY, Chen R, et al. Personal omics profiling reveals dynamic molecular and medical phenotypes. Cell. 2012;148(6):1293-307.

Cheng S, Koch WH, Wu L. Co-development of a companion diagnostic for targeted cancer therapy. New biotechnology. 2012;29(6):682-8.

Chua EW, Kennedy M A. Current State and Future Prospects of Direct-to-Consumer Pharmacogenetics. Frontiers in pharmacology. 2012;3:152.

Chun H, Kang J, Kim KJ, Park KS, Kim HC. IT-based diagnostic instrumentation systems for personalized healthcare services. Studies in health technology and informatics. 2005;117:180-90.

Clifton L, Clifton DA, Pimentel MA, Watkinson PJ, Tarassenko L. Gaussian processes for personalized e-health monitoring with wearable sensors. IEEE transactions on bio-medical engineering. 2013;60(1):193-7.

Cohen J, Wilson A, Manzolillo K. Clinical and economic challenges facing pharmacogenomics. The pharmacogenomics journal. 2013;13(4):378-88.

Cross DS, McCarty CA, Hytopoulos E, Beggs M, Nolan N, Harrington DS, et al. Coronary risk assessment among intermediate risk patients using a clinical and biomarker based algorithm developed and validated in two population cohorts. Current medical research and opinion. 2012;28(11):1819-30.

Dawe DE, Ellis PM. Challenges in implementing personalized medicine for lung cancer within a national healthcare system. Journal of Personalized M edicine. 2012;2(3):77-92. 
de Leon J. AmpliChip CYP450 test: personalized medicine has arrived in psychiatry. Expert review of molecular diagnostics. 2006;6(3):277-86.

Dolan ME, M aitland ML, O'Donnell PH, Nakamura Y, Cox NJ, Ratain MJ. Institutional Profile: University of Chicago Center for Personalized Therapeutics: research, education and implementation science. Pharmacogenomics. 2013;14(12):1383-7.

Donnan JR, Ungar WJ, Mathews M, Hancock-Howard RL, Rahman P. A cost effectiveness analysis of thiopurine methyltransferase testing for guiding 6-mercaptopurine dosing in children with acute lymphoblastic leukemia. Pediatric blood \& cancer. 2011;57(2):231-9.

Donnelly E. XalKori: A new paradigm of therapeutic and companion diagnostic co-development. Regulatory Rapporteur. 2013;10(2):9-11.

Dotan ZA, Ramon J. Nomograms as a Tool in Predicting Prostate Cancer Prognosis. European Urology, Supplements. 2009;8(9):721-4.

Dowd SE, Wolcott RD, Kennedy J, Jones C, Cox SB. M olecular diagnostics and personalised medicine in wound care: assessment of outcomes. Journal of wound care. 2011;20(5):232, 4-9.

Ellingson RM, Helt WJ, Kelt PV, Fausti SA. Instrumentation system upgrade supports mobile personalized healthcare delivery. Conference proceedings: Annual International Conference of the IEEE Engineering in Medicine and Biology Society IEEE Engineering in M edicine and Biology Society Conference. 2006;1:6233-6.

Ely S. Personalized medicine: individualized care of cancer patients. Translational research : the journal of laboratory and clinical medicine. 2009;154(6):303-8.

Erskine KE, Griffith E, Degroat N, Stolerman M, Silverstein LB, Hidayatallah N, et al. An interdisciplinary approach to personalized medicine: case studies from a cardiogenetics clinic. Personalized medicine. 2013;10(1):73-80.

Farrugia G, Weinshilboum RM . Challenges in implementing genomic medicine: the Mayo Clinic Center for Individualized Medicine. Clinical pharmacology and therapeutics. 2013;94(2):204-6.

Faruki H, Lai-Goldman M. Application of a pharmacogenetic test adoption model to six oncology biomarkers. Personalized M edicine. 2010;7(4):441-50.

Ferrusi IL, Earle CC, Trudeau M, Leighl NB, Pullenayegum E, Khong H, et al. Closing the personalized medicine information gap: HER2 test documentation practice. The American journal of managed care. 2013;19(1):838-44.

Gambacorti-Passerini C. Part I: M ilestones in personalised medicine--imatinib. The lancet oncology. 2008;9(6):600.

Gardiner SJ, Begg EJ. Pharmacogenetic testing for drug metabolizing enzymes: Is it happening in practice? Pharmacogenetics and Genomics. 2005;15(5):365-9.

Gelmon K. Part II: Milestones in personalised medicine--trastuzumab. The lancet oncology. 2008;9(7):698.

Gharani N, Keller MA, Stack CB, Hodges LM, Schmidlen TJ, Lynch DE, et al. The Coriell personalized medicine collaborative pharmacogenomics appraisal, evidence scoring and interpretation system. Genome medicine. 2013;5(10):93.

Gibson G. Wellness and health omics linked to the environment: the WHOLE approach to personalized medicine. Advances in experimental medicine and biology. 2014;799:1-14.

Glurich I, Acharya A, Shukla SK, Nycz GR, Brilliant MH. The oral-systemic personalized medicine model at M arshfield Clinic. Oral diseases. 2013;19(1):1-17. 
Gollust SE, Gordon ES, Zayac C, Griffin G, Christman M F, Pyeritz RE, et al. Motivations and perceptions of early adopters of personalized genomics: perspectives from research participants. Public health genomics. 2012;15(1):22-30.

Gordon ES, Griffin G, Wawak L, Pang H, Gollust SE, Bernhardt BA. "It's not like judgment day": public understanding of and reactions to personalized genomic risk information. Journal of genetic counseling. 2012;21(3):423-32.

Gottesman O, Scott SA, Ellis SB, Overby CL, Ludtke A, Hulot JS, et al. The CLIPM ERGE PGx Program: clinical implementation of personalized medicine through electronic health records and genomics-pharmacogenomics. Clinical pharmacology and therapeutics. 2013;94(2):214-7.

Gundert-Remy U, Dimovski A, Gajovic S. Personalized medicine - where do we stand? Pouring some water into wine: a realistic perspective. Croatian medical journal. 2012;53(4):314-20.

Gundlapalli AV, Delgado JC, Jackson BR, Tricot GJ, Hill HR. Composite patient reports: a laboratory informatics perspective and pilot project for personalized medicine and translational research. Summit on translational bioinformatics. 2009;2009:39-43.

Guo Y, Shi L, Hong H, Su Z, Fuscoe J, Ning B. Studies on abacavir-induced hypersensitivity reaction: a successful example of translation of pharmacogenetics to personalized medicine. Science China Life sciences. 2013;56(2):119-24.

Hartzler A, McCarty CA, Rasmussen LV, Williams MS, Brilliant M, Bowton EA, et al. Stakeholder engagement: a key component of integrating genomic information into electronic health records. Genetics in medicine : official journal of the American College of Medical Genetics. 2013;15(10):792-801.

Hawkins-Taylor C, Carlson AM. Communication strategies must be tailored to a medication's targeted population: Lessons from the case of BiDil. American Health and Drug Benefits. 2013;6(7):401-12.

Heidbuchel $\mathrm{H}$. Telemonitoring of implantable cardiac devices: hurdles towards personalised medicine. Heart (British Cardiac Society). 2011;97(11):931-9.

Herman L, Froelich J, Kanelos D, St Amant R, Yau M, Rhees B, et al. Utility of a Genomic-based, Personalized Medicine Test in Patients Presenting With Symptoms Suggesting Coronary Artery Disease. Journal of the American Board of Family M edicine : JABFM . 2014;27(2):258-67.

Housman L. Innovative tests for patient-tailored treatments: Demonstrating value in the age of personalized medicine. Personalized M edicine. 2011;8(2):183-9.

Hresko A, Haga SB. Insurance coverage policies for personalized medicine. Journal of Personalized Medicine. 2012;2(4):201-16.

Huang RS, Gamazon ER. Translating pharmacogenomics discoveries into the clinic: an implementation framework. Genome medicine. 2013;5(10):94.

Hulse NC, Ranade-Kharkar P, Post H, Wood GM, Williams MS, Haug PJ. Development and early usage patterns of a consumer-facing family health history tool. AMIA Annual Symposium proceedings / AM IA Symposium AM IA Symposium. 2011;2011:578-87.

Issa AM, Weiss LT, Tufail W, Phillips KA, Van Bebber SL. Innovation in personalized medicine: BiDil as a case study for integrating clinical and policy developments. Personalized Medicine. 2006;3(4):421-7.

Jain KK. Applications of AmpliChip CYP450. Molecular diagnosis : a journal devoted to the understanding of human disease through the clinical application of molecular biology. 2005;9(3):119-27. 
Jamshidi N, Miller FJ, Mandel J, Evans T, Kuo MD. Individualized therapy of HHT driven by network analysis of metabolomic profiles. BM C systems biology. 2011;5:200.

Janssens AC, Wilde AA, van Langen IM. The sense and nonsense of direct-to-consumer genetic testing for cardiovascular disease. Netherlands heart journal : monthly journal of the Netherlands Society of Cardiology and the Netherlands Heart Foundation. 2011;19(2):85-8.

Jethwani K, Kvedar J. Behavioral phenotyping: A tool for personalized medicine. Personalized Medicine. 2010;7(6):689-93.

Jiang $Y$, Wang $M$. Personalized medicine in oncology: tailoring the right drug to the right patient. Biomarkers in medicine. 2010;4(4):523-33.

Jing X, Kay S, Marley T, Hardiker NR, Cimino JJ. Incorporating personalized gene sequence variants, molecular genetics knowledge, and health knowledge into an EHR prototype based on the Continuity of Care Record standard. Journal of biomedical informatics. 2012;45(1):82-92.

Johnson JA, Burkley BM, Langaee TY, Clare-Salzler MJ, Klein TE, Altman RB. Implementing personalized medicine: development of a cost-effective customized pharmacogenetics genotyping array. Clinical pharmacology and therapeutics. 2012;92(4):437-9.

June $\mathrm{CH}$. Adoptive $\mathrm{T}$ cell therapy for cancer in the clinic. The Journal of clinical investigation. 2007;117(6):1466-76.

Kanodia AK, Kim I, Sturmberg JP. A personalized systems medicine approach to refractory rumination. Journal of evaluation in clinical practice. 2011;17(3):515-9.

Karczewski KJ, Tirrell RP, Cordero P, Tatonetti NP, Dudley JT, Salari K, et al. Interpretome: a freely available, modular, and secure personal genome interpretation engine. Pacific Symposium on Biocomputing Pacific Symposium on Biocomputing. 2012:339-50.

Kazak AE. Pediatric Psychosocial Preventative Health Model (PPPHM): Research, practice, and collaboration in pediatric family systems medicine. Families, Systems and Health. 2006;24(4):38195.

Keller M A, Gordon ES, Stack CB, Gharani N, Sill CJ, Schmidlen TJ, et al. Coriell personalized medicine collaborative: A prospective study of the utility of personalized medicine. Personalized Medicine. 2010;7(3):301-17.

Kelley RK, Van Bebber SL, Phillips KA, Venook AP. Personalized medicine and oncology practice guidelines: a case study of contemporary biomarkers in colorectal cancer. Journal of the National Comprehensive Cancer Network : JNCCN. 2011;9(1):13-25.

Khan NA, Peterson JF. A surveillance tool to support quality assurance and research in personalized medicine. AMIA Annual Symposium proceedings / AMIA Symposium AMIA Symposium. 2011;2011:701-8.

Kondylakis H, Kazantzaki E, Koumakis L, Genitsaridi I, M arias K, Gorini A, et al. Development of interactive empowerment services in support of personalised medicine. Ecancermedicalscience. 2014;8:400.

Kotze MJ, Van Velden DP, Botha K, Badenhorst CH, Avenant H, Van Rensburg SJ, et al. Pathologysupported genetic testing directed at shared disease pathways for optimized health in later life. Personalized M edicine. 2013;10(5):497-507.

Laerum H, Bremer S, Bergan S, Grunfeld T. A taste of individualized medicine: physicians' reactions to automated genetic interpretations. Journal of the American Medical Informatics Association : JAMIA. 2014;21(e1):e143-6. 
Lazaridis KN, M cAllister TM, Babovic-Vuksanovic D, Beck SA, Borad MJ, Bryce AH, et al. Implementing individualized medicine into the medical practice. American journal of medical genetics Part C, Seminars in medical genetics. 2014;166(1):15-23.

Lee HJ, Kang KJ, Park SH, Ju SJ, Jin M H, Park BN. Effect of Integrated Personalized Health Care System on Middle-Aged and Elderly Women's Health. Healthcare informatics research. 2012;18(3):199-207.

Lee JJ, Chu E. Oncotype DX for colon cancer: Are we ready for prime time in personalized medicine? Current Colorectal Cancer Reports. 2013;9(4):312-6.

Leopold C, Vogler S, Habl C, M antel-Teeuwisse AK, Espin J. Personalised medicine as a challenge for public pricing and reimbursement authorities - A survey among 27 European countries on the example of trastuzumab. Health policy (Amsterdam, Netherlands). 2013;113(3):313-22.

Li A, Bozdag S, Kotliarov Y, Fine HA. GliomaPredict: a clinically useful tool for assigning glioma patients to specific molecular subtypes. BMC medical informatics and decision making. 2010;10:38.

Lieb W, Volzke H, Pulley JM, Roden DM, Kroemer HK. Strategies for personalized medicine-based research and implementation in the clinical workflow. Clinical pharmacology and therapeutics. 2012;92(4):443-5.

Lin BK, Edelman E, M clnerney JD, O'Leary J, Edelson V, Hughes KS, et al. Personalizing prenatal care using family health history: Identifying a panel of conditions for a novel electronic genetic screening tool. Personalized M edicine. 2013;10(3):307-18.

Linardou H, Briasoulis E, Dahabreh IJ, M ountzios G, Papadimitriou C, Papadopoulos S, et al. All about KRAS for clinical oncology practice: gene profile, clinical implications and laboratory recommendations for somatic mutational testing in colorectal cancer. Cancer treatment reviews. 2011;37(3):221-33.

Li-Pook-Than J, Snyder M. iPOP goes the world: integrated personalized Omics profiling and the road toward improved health care. Chemistry \& biology. 2013;20(5):660-6.

Litton G, Kane D, Clay G, Kruger P, Belnap T, Parkinson B. M ultidisciplinary cancer care with a patient and physician satisfaction focus. Journal of oncology practice / American Society of Clinical Oncology. 2010;6(6):e35-7.

Lorenz M, Klemm M, Eidens M, Fleischer M, Weise A, Braun W, et al. Actionable nutrigenetics for genetically based diseases? a new critical path to P4 medicine. Current Pharmacogenomics and Personalized M edicine. 2013;11(2):167-80.

Love D, Stratton E, Stocum M. Best practices for companion diagnostic and therapeutic development: Translating between the stakeholders. New Biotechnology. 2012;29(6):689-94.

MacDonald DJ, Blazer KR, Weitzel JN. Extending comprehensive cancer center expertise in clinical cancer genetics and genomics to diverse communities: the power of partnership. Journal of the National Comprehensive Cancer Network : JNCCN. 2010;8(5):615-24.

Mascarin M, Truccolo I, Byther E, Capone D, Elia C. Cancer, Adolescence, and Their Peers: "They'll give you a Story". Journal of cancer education : the official journal of the American Association for Cancer Education. 2014.

McClure JB, Swan GE, St John J, Fauver R, Javitz HS, Bergen AW, et al. Pharmacogenetic smoking cessation intervention in a health care setting: a pilot feasibility study. Nicotine $\&$ tobacco research : official journal of the Society for Research on Nicotine and Tobacco. 2013;15(2):51826.

M eckley LM, Neumann PJ. Personalized medicine: factors influencing reimbursement. Health policy (Amsterdam, Netherlands). 2010;94(2):91-100. 
Meier RJ. A critique of race-based and genomic medicine. Collegium antropologicum. 2012;36(1):510.

Meric-Bernstam F, Farhangfar C, Mendelsohn J, Mills GB. Building a personalized medicine infrastructure at a major cancer center. Journal of clinical oncology : official journal of the American Society of Clinical Oncology. 2013;31(15):1849-57.

M erlin T, Farah C, Schubert C, Mitchell A, Hiller JE, Ryan P. Assessing personalized medicines in Australia: a national framework for reviewing codependent technologies. M edical decision making : an international journal of the Society for Medical Decision Making. 2013;33(3):333-42.

M ini E, Nobili S. Pharmacogenetics: implementing personalized medicine. Clinical cases in mineral and bone metabolism : the official journal of the Italian Society of Osteoporosis, Mineral M etabolism, and Skeletal Diseases. 2009;6(1):17-24.

$\mathrm{Ng} \mathrm{PC}$, Murray SS, Levy S, Venter JC. An agenda for personalized medicine. Nature. 2009;461(7265):724-6.

Nishimura T, Kato H, Ikeda N, Kihara M, Nomura M, Kato Y, et al. Cancer Phenotype Diagnosis and Drug Efficacy within Japanese Health Care. International journal of proteomics. 2012;2012:921901.

O'Donnell PH, Danahey K, Jacobs M, Wadhwa NR, Yuen S, Bush A, et al. Adoption of a clinical pharmacogenomics implementation program during outpatient care-initial results of the University of Chicago "1,200 Patients Project". American journal of medical genetics Part C, Seminars in medical genetics. 2014;166(1):68-75.

Ong FS, Das K, Wang J, Vakil H, Kuo JZ, Blackwell WL, et al. Personalized medicine and pharmacogenetic biomarkers: progress in molecular oncology testing. Expert review of molecular diagnostics. 2012;12(6):593-602.

O'Rawe JA, Fang H, Rynearson S, Robison R, Kiruluta ES, Higgins G, et al. Integrating precision medicine in the study and clinical treatment of a severely mentally ill person. Peer]. 2013;1:e177.

Orlando LA, Hauser ER, Christianson C, Powell KP, Buchanan AH, Chesnut B, et al. Protocol for implementation of family health history collection and decision support into primary care using a computerized family health history system. BM C health services research. 2011;11:264.

Orlando LA, Henrich VC, Hauser ER, Wilson C, Ginsburg GS. The genomic medicine model: An integrated approach to implementation of family health history in primary care. Personalized Medicine. 2013;10(3):295-306.

Orlando LA, Wu RR, Beadles C, Himmel T, Buchanan AH, Powell KP, et al. Implementing family health history risk stratification in primary care: Impact of guideline criteria on populations and resource demand. American journal of medical genetics Part C, Seminars in medical genetics. 2014;166(1):24-33.

Ou SH, Bartlett CH, Mino-Kenudson M, Cui J, lafrate AJ. Crizotinib for the treatment of ALKrearranged non-small cell lung cancer: a success story to usher in the second decade of molecular targeted therapy in oncology. The oncologist. 2012;17(11):1351-75.

Overby $\mathrm{CL}$, Erwin AL, Abul-Husn NS, Ellis SB, Scott SA, Obeng AO, et al. Physician attitudes toward adopting genome-guided prescribing through clinical decision support. Journal of Personalized Medicine. 2014;4(1):35-49.

Overby CL, Tarczy-Hornoch P, Hoath JI, Kalet IJ, Veenstra DL. Feasibility of incorporating genomic knowledge into electronic medical records for pharmacogenomic clinical decision support. BM C bioinformatics. 2010;11 Suppl 9:S10.

Palmer JE. Genetic gatekeepers: regulating direct-to-consumer genomic services in an era of participatory medicine. Food and drug law journal. 2012;67(4):475-524, iii. 
Patel CJ, Sivadas A, Tabassum R, Preeprem T, Zhao J, Arafat D, et al. Whole genome sequencing in support of wellness and health maintenance. Genome medicine. 2013;5(6):58.

Phillips KA. The intersection of biotechnology and pharmacogenomics: health policy implications. Health affairs (Project Hope). 2006;25(5):1271-80.

Phillips KA, Liang SY, Van Bebber S. Challenges to the translation of genomic information into clinical practice and health policy: Utilization, preferences and economic value. Current opinion in molecular therapeutics. 2008;10(3):260-6.

Piatkov I, Jones T, Rochester C. Cytochrome P450 loss-of-function polymorphism genotyping on the Agilent Bioanalyzer and clinical application. Pharmacogenomics. 2009;10(12):1987-94.

Platt D. When consumers get their genomes. Personalized M edicine. 2009;6(6):669-79.

Poh N, de Lusignan S. Data-modelling and visualisation in chronic kidney disease (CKD): a step towards personalised medicine. Informatics in primary care. 2011;19(2):57-63.

Printz $\mathrm{C}$. Universities bring personalized medicine to the forefront of patient care: two programs aim to make customized treatments easily accessible. Cancer. 2012;118(10):2563-4.

Pulley JM, Denny JC, Peterson JF, Bernard GR, Vnencak-Jones CL, Ramirez AH, et al. Operational implementation of prospective genotyping for personalized medicine: the design of the Vanderbilt PREDICT project. Clinical pharmacology and therapeutics. 2012;92(1):87-95.

Richards M. Reading the runes of my genome: A personal exploration of retail genetics. New Genetics and Society. 2010;29(3):291-310.

Ritchie MD. The success of pharmacogenomics in moving genetic association studies from bench to bedside: study design and implementation of precision medicine in the post-GWAS era. Human genetics. 2012;131(10):1615-26.

Rosenbloom ST, Daniels TL, Talbot TR, McClain T, Hennes R, Stenner S, et al. Triaging patients at risk of influenza using a patient portal. Journal of the American Medical Informatics Association : JAM IA. 2012;19(4):549-54.

Roychowdhury S, Iyer MK, Robinson DR, Lonigro RJ, Wu Y-M, Cao X, et al. Personalized Oncology Through Integrative High-Throughput Sequencing: A Pilot Study. Science Translational Medicine. 2011;3(111):111ra21.

Ruddy KJ, Desantis SD, Gelman RS, Wu AH, Punglia RS, Mayer EL, et al. Personalized medicine in breast cancer: tamoxifen, endoxifen, and CYP2D6 in clinical practice. Breast cancer research and treatment. 2013;141(3):421-7.

Schmidlen TJ, Wawak L, Kasper R, Garcia-Espana JF, Christman MF, Gordon ES. Personalized Genomic Results: Analysis of Informational Needs. Journal of genetic counseling. 2014.

Shackelford RE, Whitling NA, M cNab P, Japa S, Coppola D. KRAS Testing: A Tool for the Implementation of Personalized M edicine. Genes \& cancer. 2012;3(7-8):459-66.

Smarr L. Quantifying your body: a how-to guide from a systems biology perspective. Biotechnology journal. 2012;7(8):980-91.

Sohn S, Helms TM, Pelleter JT, Muller A, Krottinger Al, Schoffski O. Costs and benefits of personalized healthcare for patients with chronic heart failure in the care and education program "Telemedicine for the Heart". Telemedicine journal and e-health : the official journal of the American Telemedicine Association. 2012;18(3):198-204.

Sorich MJ, M cKinnon RA. Personalized medicine: potential, barriers and contemporary issues. Current drug metabolism. 2012;13(7):1000-6.

Sottas PE, Robinson N, Rabin O, Saugy $M$. The athlete biological passport. Clinical chemistry. 2011;57(7):969-76. 
Stack CB, Gharani N, Gordon ES, Schmidlen T, Christman MF, Keller M A. Genetic risk estimation in the Coriell Personalized Medicine Collaborative. Genetics in medicine : official journal of the American College of M edical Genetics. 2011;13(2):131-9.

Su CJ, Chiang CY. IAServ: an intelligent home care web services platform in a cloud for aging-in-place. International journal of environmental research and public health. 2013;10(11):6106-30.

Sullivan SD, Garrison LP, Jr., Rinde H, Kolberg J, M oler EJ. Cost-effectiveness of risk stratification for preventing type 2 diabetes using a multi-marker diabetes risk score. Journal of medical economics. 2011;14(5):609-16.

Sweet K, Gordon ES, Sturm AC, Schmidlen TJ, Manickam K, Tol AE, et al. Design and implementation of a randomized controlled trial of genomic counseling for patients with chronic disease. Journal of Personalized M edicine. 2014;4(1):1-19.

Tenenbaum JD, James A, Paulyson-Nunez K. An altered treatment plan based on direct to consumer (DTC) genetic testing: Personalized medicine from the patient/pin-cushion perspective. Journal of Personalized Medicine. 2012;2(4):192-200.

Teng K, Eng C, Hess CA, Holt MA, Moran RT, Sharp RR, et al. Building an innovative model for personalized healthcare. Cleveland Clinic journal of medicine. 2012;79 Suppl 1:S1-9.

Thompson R. Developing and marketing a women's health Web site. HealthlyM e.md seeks to provide personalized health care over the Internet. M arketing health services. 2000;20(3):42-4.

Topol EJ, Lauer MS. The rudimentary phase of personalised medicine: coronary risk scores. Lancet. 2003;362(9398):1776-7.

Towse A, Ossa D, Veenstra D, Carlson J, Garrison L. Understanding the economic value of molecular diagnostic tests: Case studies and lessons learned. Journal of Personalized Medicine. 2013;3(4):288-305.

Trosman JR, Van Bebber SL, Phillips KA. Coverage policy development for personalized medicine: private payer perspectives on developing policy for the 21-gene assay. Journal of oncology practice / American Society of Clinical Oncology. 2010;6(5):238-42.

Vaiopoulou A, Gazouli M, Karikas GA. Pharmacogenomics: current applications and future prospects towards personalized therapeutics. Journal of BUON : official journal of the Balkan Union of Oncology. 2013;18(3):570-8.

Van Allen EM, Foye A, Wagle N, Kim W, Carter SL, McKenna A, et al. Successful whole-exome sequencing from a prostate cancer bone metastasis biopsy. Prostate Cancer and Prostatic Diseases. 2014;17(1):23-7.

van't Veer LJ, Bernards R. Enabling personalized cancer medicine through analysis of geneexpression patterns. Nature. 2008;452(7187):564-70.

Vizirianakis IS. Improving pharmacotherapy outcomes by pharmacogenomics: from expectation to reality? Pharmacogenomics. 2005;6(7):701-11.

Vorderstrasse AA, Ginsburg GS, Kraus WE, Maldonado MC, Wolever RQ. Health coaching and genomics-potential avenues to elicit behavior change in those at risk for chronic disease: protocol for personalized medicine effectiveness study in air force primary care. Global advances in health and medicine : improving healthcare outcomes worldwide. 2013;2(3):26-38.

Wac K. Smartphone as a personal, pervasive health informatics services platform: literature review. Yearbook of medical informatics. 2012;7(1):83-93.

Wang X, Gui Q, Liu B, Jin Z, Chen Y. Enabling Smart Personalized Healthcare: a Hybrid Mobile-Cloud Approach for ECG Telemonitoring. IEEE journal of biomedical and health informatics. 2013. 
Wang YD, Phillips-Wren G, Forgionne G. E-delivery of personalised healthcare information to intermediaries for suicide prevention. International journal of electronic healthcare. 2005;1(4):396412.

Waring PM. Matching patients with drugs: Triumphs and challenges. Personalized Medicine. 2006;3(3):335-44.

Weitzel KW, Elsey AR, Langaee TY, Burkley B, Nessl DR, Obeng AO, et al. Clinical pharmacogenetics implementation: Approaches, successes, and challenges. American journal of medical genetics Part C, Seminars in medical genetics. 2014;166(1):56-67.

Weldon CB, Trosman JR, Gradishar WJ, Benson AB, 3rd, Schink JC. Barriers to the use of personalized medicine in breast cancer. Journal of oncology practice / American Society of Clinical Oncology. 2012;8(4):e24-31. 
It is more important to know what sort of person has a disease than to know what sort of disease a person has.

Hippocrates 


\section{Chapter 5}

\section{A perspective on personalized health care}

Tomris Cesuroglu, Ben van Ommen, Nuria Malats, Ralph Sudbrak, Hans Hans Lehrach and Angela Brand Public health perspective: from personalized medicine to personal health. Personalized Medicine. 2012;9(2):115-9. 
A gradual shift from a 'one-size-fits-all' strategy towards personalized interventions is observed in the whole spectrum of healthcare, including personalized prevention, diagnosis and therapy, and the concept of personal health. This shift is caused by two major drivers. The first one is a general societal trend towards 'personalization', where individuals demand services or products that are customized to their needs, possibilities and choices. The second driver is rapid scientific and technological advancements in genomebased science and technologies (which is a term that covers the full breadth of knowledge that is being amassed in all 'omics fields [1, 2]), information, and information and communication technologies (ICT).

The future paradigm in health and healthcare has become increasingly visible in the past 10-15 years and has been commonly defined as 'personalized medicine' although the exact definition varies among different stakeholders and opinion leaders. In the ongoing communications, the term personalized medicine comprises two separate and independent approaches. The first approach is based on biomarkers and statistical methods subdividing a heterogeneous group into smaller less heterogeneous groups leading to stratified medicine. The stratification into smaller and smaller subgroups is limited by the statistical power, and may therefore, not incrementally lead to individualization. The other approach is based on 'omics and other data obtained from the individual in conjunction with computer modeling approaches leading to 'individualized medicine'.

'Personalized' mostly refers to the use of genome-based information and technologies for providing more stratified (and possibly personalized) interventions. Some authors put the emphasis not only on medicine but on healthcare as a whole and prefer the term 'personalized healthcare', which is mostly used interchangeably with personalized medicine. However, personalized healthcare is much broader in its scope and covers all interventions in a healthcare system including interventions in the medical, as well as, public health setting [3].

The publication of the initial draft and final version of the Human Genome Project in the early 2000s created high expectations for 'genome-based' medicine and personalized medicine. But only with the intervention of the new sequencing technologies termed nextgeneration sequencing, it was possible to analyze the genomes of individuals in larger numbers. Although it is now evident that genomic information has revolutionized our understanding of (human) biology, its translation in drug discovery and medical practice has not been straightforward. So far 'genome-based' personalized medicine has provided limited output that can be translated into practice. This led to questioning whether personalization of medicine with genome-based information is a 'myth' or reality [4, 5]. We now recognize that the last decade was more about understanding the biology of genomes, whereas the coming decade will bring more information for understanding the biology of diseases [6]. Clear examples of advancing the science of medicine and improving the effectiveness of healthcare using genome-based information can be expected to 
start to flourish in this decade, but will probably become more prominent in the period beyond the year 2020 [6].

In addition to genome-based personalization, there are other drivers that will mark the future trends and opportunities in medicine and healthcare, such as prediction by using early markers of progression from a healthy state to disease, prevention by personalized early diagnosis and targeting the specific mechanisms that are determining the disease progression in each individual, and participation of the individual in decision-making and management of the disease. Together, these aspects shape predictive, preventive, personalized and participatory (P4) Medicine [7]. It is of note that developments in imaging and sensor technologies will also play an important role in the near future to individualize medicine and healthcare.

There are several issues and challenges in the journey towards this future paradigm of medicine and healthcare. In this commentary, we are addressing some of them that might be critical on the way from genome-based discoveries to real-life implementation with a public health perspective.

\section{Interaction among factors that surround the individual}

In the initial comments on personalized medicine, genomic information of individuals was the main focus. This was interpreted commonly by media and some scientists in a way that the genome, which is unique for every individual, would be the ultimate means for individualization. The truth here is both shocking and simple: our genome is not the only factor that makes us unique. As genome science evolves, the old truth that the phenotype is shaped by interaction of our genotype with our environment re-emerges and straightforward interpretation of genotypic variation into personalized medicine is 'confounded' by multiple environmental interactions. Often genotypic variations turn out to be only minor contributors to disease etiology. The environmental factors surrounding individuals, such as exposure to pathogens or toxins, socioeconomic factors, physical activity, nutrition and other lifestyle factors are constantly interacting with each other, as well as with the genomes. As science progresses, the importance of understanding these genomeenvironment interactions is getting more and more important. We are moving towards a new understanding of health and disease, showing that these genome-environment interactions change from day-to-day within an individual. Neither genomics nor the environment is stable. Biological pathways or networks are permanently interacting with environmental networks such as social networks. Thus, a comprehensive model of future healthcare taking into account integrative genomics alongside environmental, social and lifestyle factors will become essential to realize the P4 M edicine as the future paradigm of healthcare systems. 
Specific examples of environmental factors are the 'exposome' of individuals, representing the combined exposures from all sources that reach the internal chemical environment, and can be measured as metals in toenails [8, 9]. Pathogens are also strong external factors that lead to disease. A prominent example of interaction of pathogens and the host genome is human papilloma virus and cervical cancer, whereby a pathogen may cause cervical cancer although 'cancer' is a disease traditionally classified as 'noncommunicable'. Moreover, one of the factors that determines if and when progression to the disease will take place is the host's genomic background [10]. The future is likely to show us more and more of such examples.

The interaction of genome and lifestyle, as well as other environmental factors, may become more visible by studying epigenomics. Epigenomics may also provide insights to interactions with socioeconomical factors and play a crucial role for public health by bridging social and biomedical sciences [11]. For example, nightshift work, which can be categorized as a 'lifestyle' or 'socioeconomical' factor, increases the risk of breast cancer [12]. This effect may be mediated by an epigenomic change in circadian rhythm genes and 'night shift' may also turn into an inherited risk factor for breast cancer with potential effect for future generations [13].

\section{From genomic to systems approaches in personalized health}

The path to evolve from genome-based personalized medicine to personalized medicine that takes all the relevant factors into account is the transition from genomic to systems approaches in personalized health. Systems biology is defined as an integrative, interdisciplinary approach to biological science that is built around the concept of close integration of computational methods, technology development and global measurement and analysis of biological systems [14]. The integrative approach of systems biology has so far focused on the biological determinants of health and integrates various levels of information for discovery of disease causing biological networks. For example, nutritional systems biology [15] integrates 'nutrition', which is classified as a 'lifestyle factor', into systems biology studies by taking into account all biological mechanisms and processes that are affected by dietary components on all relevant levels of complexity. Integration of other determinants of health into systems biology approaches may provide further insight to understanding the biology of health and diseases. This approach is supported by the Public Health Genomics European Network (PHGEN), which is currently producing the first edition of "European Best Practice Guidelines for Quality Assurance, Provision and Use of Genome-based Information and Technologies" assisting all EU Member States [European Commission. European best practice guidelines for quality assurance, provision and use of genome-based information and technologies (2012), Policy report in preparation] [16, 17].

The integrative approach should not only be a concept for scientific discoveries, but also a main constituent of personalized medicine approaches in practice. In order to manage an 
individual's health, disease risks and diseases, we need to involve all available information related to the individual; make a 360-degree assessment of the individual's health, including personal, health, socioeconomic and lifestyle information, biomarkers and 'omics information; develop a personalized plan; and follow-up the progress made by the individual [18]. There are published and unpublished models indicating similar integrative approaches, which are conceptually developed $[3,19]$, prepared for implementation or developed as a practice model and implemented as a pilot study [18]. It is likely that more integrative approaches will soon emerge around the globe.

\section{Role of the individual}

While various attempts are made to understand and manage health, disease risk and diseases, the perspective and expectations of the individual, who is in the center of all, should also be taken into account. Individuals are taking more and more control of their health because they have better access to health-related information and a higher desire to control their own life and health. It is not uncommon anymore to hear about patients who challenge their healthcare professionals with information they have found on the internet or obtained from their 'peers' with the same condition and/or symptoms. Social networking platforms such as PatientsLikeM e [20] enable its members to share condition, treatment and symptom information in order to monitor their health over time and learn from real-world outcomes. Furthermore, recently Sage Bionetworks launched a project called 'Portable Legal Consent' being a tool for patients to tell doctors, researchers, companies and other stakeholders in the healthcare system, that they, the patients, own the rights to the data generated from their bodies. Portable Legal Consent states that "what the patient desires is for the data to be shared broadly in the public domain, to serve scientific progress as a whole, regardless of the particular individual or institution that makes the breakthrough" [21].

Individuals should have the lifelong skills to find and assess the relevant and reliable information on the internet, which is also related to the concept of health literacy. In Europe the consortium of the European Health Literacy Project (HLS-EU) [22] defined four dimensions of health literacy. Using this definition health literacy can be used as a catalyst to accelerate the accessibility, understandability, appraisal and application of genome-based information matching the needs within different population groups. In this context, examples such as Google [23] provide a powerful health literacy friendly electronic environment facilitating self-management, simplicity and user-centered applications. Thus, the future patient may turn from a passive consumer of health interventions into a proactive consumer or 'prosumer' [24, 25]. 


\section{ICT as facilitating factors}

ICT plays a central role in the implementation of personalized medicine in real life at several points. The development of a new, data-rich, individualized medicine is likely to surpass the demands of all other ICT development fields. As data-intensive analysis and computer-intensive modeling technologies become common clinical practice, ICT capacity and organization will become key limiting factors in medicine, resulting in shifts of resources from personnel intensive to ICT intensive application. Data-rich, individualized medicine poses unprecedented challenges for ICT, in hardware, storage and communication.

The combined genomic and phenotypic analysis may become very complex and needs algorithms and mathematical models to provide a clear diagnosis and therapy. A doctor's computational brainpower is not enough to process all of the different factors surrounding the individual where there are hundreds or maybe even thousands of data to be considered, make current status and risk assessments, and based on these, draw personal plans for management of the individual's health, risks and diseases. ICT is also essential in optimal coaching and patient empowering, both in complicated therapies (compliance) and in lifestyle coaching for prevention and health optimization. In order to facilitate a healthcare professional's adoption of personalized medicine, we need strong information and communication platforms integrated into healthcare services to collect and analyze data, assess and interpret it, develop personal plans, follow-up the implementation and provide updates and revisions to the plan. At the same time, this should be a closed circuit that feeds science in identifying new hazards, both environmental and genomics, for disease risk and progression [26].

The IT Future of Medicine (ITFoM) provides such a platform for Europe being one of six pilot projects in the European Future and Emerging Technologies Flagship scheme [27]. It produces computational models of individual persons ('the virtual twin'), which will follow the person during the whole life course through the healthcare systems enabling healthcare professionals to virtually simulate and optimize treatments. In the end, medical decision-making may turn into in silico decision-making.

\section{Conclusion}

There are several opportunities and challenges on the way to the future vision of medicine and healthcare. One of the key factors for its realization is aiming for an integrative approach towards an individual's health, risks and diseases, considering all relevant factors surrounding them, such as genome, stages of life (from preconception to old age), exposure to pathogens, nutrition, toxins, physical activity and other lifestyle factors, social interactions, stress and other socioeconomic factors, and healthcare systems. ICT will be critically important at all phases of this journey, including scientific discovery and translation and application to healthcare practices. 


\section{Future perspective}

Personal health drives a fundamental change not just in what is known, but also in how we think of ourselves and the way we are living, thus redefining our society. The political will is there, but we have to prepare for all the various organizational changes. The real paradigm shift depends on the willingness to restructure policies. For realization of P4 Medicine, there is a clear urgency to prepare healthcare systems and policy makers in time.

\section{References}

1. Genome-based Research and Population Health. Report of an expert workshop held at the Rockefeller Foundation Study and Conference Centre Bellagio, Italy, 14-20 April 2005: Public Health Genetics Unit, Cambridge, UK; Institute for Public Health Genetics, University of Washington, Seattle, USA; Office of Genomics \& Disease Prevention, Center for Disease Control and Prevention, Atlanta, USA 2005. Available from: http://www.phgen.eu/typo3/fileadmin/downloads/bellagio011807.pdf.

2. Burke W, Khoury MJ, Stewart A, Zimmern RL. The path from genome-based research to population health: development of an international public health genomics network. Genet Med. 2006 Jul;:8(7):451-8.

3. Simmons LA, Dinan MA, Robinson TJ, Snyderman R. Personalized medicine is more than genomic medicine: confusion over terminology impedes progress towards personalized healthcare. Personalized M edicine. 2011 2012/01/01;9(1):85-91.

4. Emanuel E, editor Is Personalized Medicine a Myth? (Key note presentation). 7th Annual Personalized Medicine Conference; 2011 09-10 November 2012; The Joseph B. Martin Conference Center, Harvard Medical School, Boston, MA, USA: Partners HealthCare Center for Personalized Genetic Medicine.

5. Greenberg T. Conference Scene: Personalized Medicine comes to Harvard. Personalized Medicine. 2011 2012/01/01;9(1):17-8.

6. Green ED, Guyer MS. Charting a course for genomic medicine from base pairs to bedside. Nature. 2011 Feb 10;470(7333):204-13.

7. Hood L, Friend SH. Predictive, personalized, preventive, participatory (P4) cancer medicine. Nat Rev Clin Oncol. 2011 M ar;8(3):184-7.

8. Rappaport SM , Smith MT. Epidemiology. Environment and disease risks. Science. 2010 Oct 22;330(6003):460-1. PubM ed PM ID: 20966241. Epub 2010/10/23.

9. Amaral AF, Porta M, Silverman DT, Milne RL, Kogevinas M, Rothman N, et al. Pancreatic cancer risk and levels of trace elements. Gut. 2011 Dec 19.

10. Wang SS, Gonzalez P, Yu K, Porras C, Li Q, Safaeian M, et al. Common genetic variants and risk for HPV persistence and progression to cervical cancer. PLoS One. 2010;5(1):e8667.

11. Brand A. Public health genomics--public health goes personalized? Eur J Public Health. 2011 Feb;21(1):2-3.

12. Schernhammer ES, Laden F, Speizer FE, Willett WC, Hunter DJ, Kawachi I, et al. Rotating night shifts and risk of breast cancer in women participating in the nurses' health study. J Natl Cancer Inst. 2001 Oct 17;93(20):1563-8.

13. Hoffman $A E, Y i C H$, Zheng $T$, Stevens RG, Leaderer $D$, Zhang $Y$, et al. CLOCK in breast tumorigenesis: genetic, epigenetic, and transcriptional profiling analyses. Cancer Res. 2010 Feb 15;70(4):1459-68. 
14. Galas DJ, Hood L. Systems Biology and Emerging Technologies Will Catalyze the Transition from Reactive Medicine to Predictive, Personalized, Preventive and Participatory (P4) Medicine. Interdisciplinary Bio Central. 2009;1(2):1-4.

15. van Ommen B. Personalized nutrition from a health perspective: Luxury or necessity? Genes Nutr. 2007 Oct;2(1):3-4.

16. Coggi PT. A European view on the future of personalised medicine in the EU. Eur J Public Health. 2011 Feb;21(1):6-7.

17. Public Health Genomics European Network - Official Website [cited 201231 January 2012]. Available from: http://www.phgen.eu/typo3/index.php.

18. Cesuroglu T, Karaca S, Erge S. A practice model for personalized healthcare with a public health genomics perspective. Personalized M edicine. 2009 2009/09/01;6(5):567-77.

19. Bousquet J, Anto JM, Sterk PJ, Adcock IM, Chung KF, Roca J, et al. Systems medicine and integrated care to combat chronic noncommunicable diseases. Genome Med. 2011;3(7):43.

20. PatientsLikeM e [31 January 2012]. Available from: www.patientslikeme.com.

21. Portable legal consent - WeConsent.us [31 January 2012]. Available from: http://weconsent.us/ faq.

22. HLS-EU: European health literacy survey [31 January 2012]. Available from: www.healthliteracy.eu

23. Google - Search Engine [31 January 2012]. Available from: www.google.com.

24. Brand $A$, Brand $H$. Health literacy and public health genomics: innovation management by citizens. Public Health Genomics. 2011;14(4-5):193-4.

25. Sorensen $\mathrm{K}$, Brand $\mathrm{H}$. Health literacy: the essential catalyst for the responsible and effective translation of genome-based information for the benefit of population health. Public Health Genomics. 2011;14(4-5):195-200.

26. National Research Council (US) Committee on A Framework for Developing a New Taxonomy of Disease. Toward Precision Medicine: Building a Knowledge Network for Biomedical Research and a New Taxonomy of Disease. Washington, DC, USA: The National Academies Press (US); 2011.

27. ITFoM - IT Future of Medicine Web Site [31 January 2012]. Available from: http://www.itfom.eu/. 
If we could give every individual the right amount of nourishment and exercise, not too little and not too much, we would have found the safest way to health.

Hippocrates 


\section{Chapter 6}

\section{Introducing a preventive personalized health care model: Gentest}

Tomris Cesuroglu, Sefayet Karaca and Sema Erge

A practice model for personalized healthcare with a public health genomics perspective. Personalized Medicine. 


\begin{abstract}
Epidemiological and demographic transition has brought populations to an extended life expectancy in the $21^{\text {st }}$ century. The diseases of this century are complex, which stem mainly from the complex interactions of the human genome with lifestyle and environmental factors. These diseases are common, chronic and costly. Currently, the best-known prevention for complex diseases is adopting a healthy lifestyle. However, this is not achieved in many places in the world. Effective intervention models, including lifestyle changes for the prevention of these diseases, are urgently needed. In this report, we introduce a preventive healthcare model based on personalized healthcare. It is based on the application of public health genomics tools and concepts on an individual level, in order to stratify individuals according to risk groups, prevent diseases and detect them early.
\end{abstract}


Life expectancy has been steadily increasing in almost all regions of the world since the beginning of the 20th Century. This happened as a result of epidemiologic and demographic transition in the world [1]. In the beginning of the 20th Century, life expectancy was below 50 years in western countries, whereas today it is above 75 years. Populations are rapidly aging.

The diseases of the $21^{\text {st }}$ century are chronic and complex, which stem mainly from the complex interaction of the human genome with lifestyle factors. Cardiovascular and cerebrovascular diseases, cancers, diabetes, obesity, osteoporosis, neurodegenerative diseases and psychiatric diseases are among major chronic and complex diseases, which account for approximately $84 \%$ of deaths and $76 \%$ of the burden of disease in high- and uppermiddle-income countries [2]. The prevalence of chronic diseases increases significantly with age. Thus, the $21^{\text {st }}$ century brings us to an aged population living with chronic conditions, creating a huge burden on healthcare systems and society. Complex diseases are not only the problem of high-income/industrialized countries, but also low-income countries in the process of industrialization.

One of the biggest challenges of the health and social systems of the $21^{\text {st }}$ century is to add productivity and life quality to prolonged life years, while keeping the healthcare costs under control by reducing the burden of complex diseases. The solution lies in preventive interventions that start in earlier ages, much earlier than the onset of the complex diseases. As the most important preventive measure, individuals must follow an appropriate and personal lifestyle plan. Giving general information and recommendations on health issues to the public has limited effectiveness to change the lifestyle of individuals. Therefore, an effective intervention model including lifestyle changes for prevention of these diseases is urgently needed.

\section{Complex diseases, genetics $\&$ personalized healthcare}

\subsection{Complex diseases \& genetics}

Complex diseases are caused by the interaction of genetics with the lifestyle of an individual. In order to understand the genetic basis of diseases, we can visualize the diseases in a threshold model, as shown in Figure 1. Here, diseases are grouped into three major areas, according to the disease-causing factors.

The first group of diseases is caused by alterations in the genetic structure of human beings. Down's syndrome and cystic fibrosis are disorders caused by deleterious changes in the genes associated with the diseases. They constitute a small fraction of the burden of disease. External factors play little or no role in most of these diseases. With today's conditions, there is limited space for measures to prevent the disease occurrence in individuals carrying a genetic abnormality (see first column in Figure 1). 
The second group of diseases is caused directly by external factors such as infectious diseases, injuries, poisonings and so on (see second column in Figure 1). Genetics has a very limited role in these conditions. This group, especially infectious diseases within it, was the major burden of disease before the industrial revolution. The burden of disease caused by this group has reduced relatively, parallel to the developments in science and technology such as sanitation, antibiotics and vaccines.

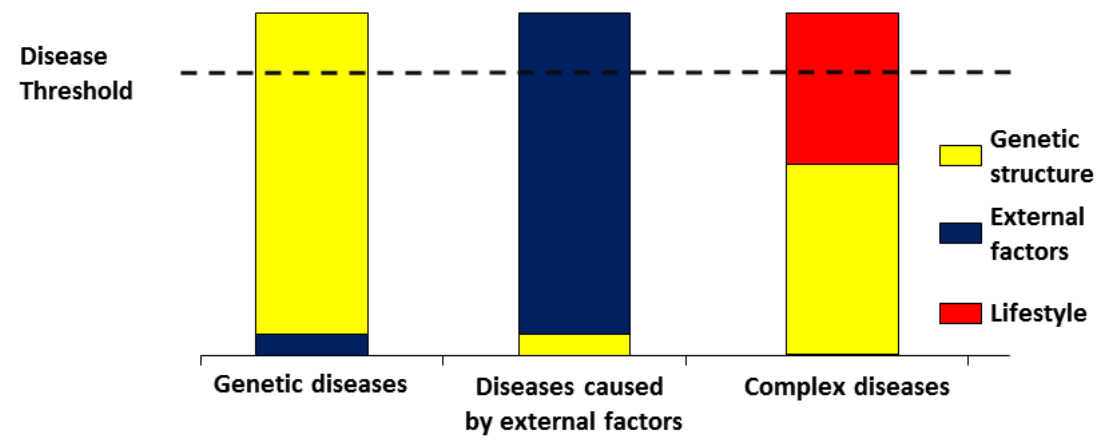

$\begin{array}{lccc}\begin{array}{l}\text { Proportion in } \\ \text { burden of disease }\end{array} & 1 \% & 39 \% & 60 \% \\ \begin{array}{l}\text { Proportion in } \\ \text { deaths }\end{array} & 1 \% & 29 \% & 70 \%\end{array}$

Figure 1: Diseases, genes, environment and lifestyle interaction from a disease threshold perspective

The third group consists of disorders where common variations in genetic structure (polymorphisms) create a predisposition to the disease, but cannot cause the disease without other factors, mainly unfavorable lifestyle factors of individuals (see third column in Figure 1). Because of this complex nature of gene-lifestyle interaction, they are called complex diseases. Main examples include cardiovascular and cerebrovascular diseases, diabetes, osteoporosis, cancers, neurological conditions and psychiatric disorders, which are mostly late-onset chronic diseases. They arise from an individual's lifestyle and environmental factors imposed on their genetic predisposition. They consist of a large proportion of burden of disease all over the world. The burden of chronic complex diseases will continue to expand steadily as a result of demographic and epidemiological transition in the next 20 years [2].

\subsection{Opportunities for the use of genetics in complex diseases}

Since the successful completion of the Human Genome Project, we have an exponentially increasing understanding of genetic factors and complex diseases. The identification of new genes and polymorphisms that have influence in diseases is helping to understand the underlying biology of the diseases, and leading to new therapeutic approaches, as well 
as understanding of how genetic variations are of influence in predisposition of different individuals to different diseases. The knowledge regarding associations of polymorphisms with complex diseases is constantly growing.

One of the success factors of nutritional interventions is prediction of the response of the individual to specific interventions. At this point, nutrigenetics is expected to play a major role. Nutrigenetics, which studies an individual's specific response to diet owing to genetic variants (polymorphisms) [3], is positioned as the emerging face of nutrition that, when considered with more classical approaches, will provide the necessary stepping stones to achieve the ambitious goal of optimizing an individual's health. Similarly, pharmacogenetics will allow us to tailor the pharmacological interventions according to the specific needs of individuals, and minimize side effects while maximizing efficacy.

The strong interaction of biological and genetic factors with lifestyle factors in the development of chronic and complex diseases has brought us to a new understanding of 'genetics', where genetics is not only related to the study of rare hereditary disorders, as understood in 'conventional' medical genetics. In the late 1990s and early 2000s, it was foreseen that genetics/genomics would revolutionize medicine [4-6], and that genetics would become a tool widely used for prediction, diagnosis and the optimization of treatment in most common diseases within the current decade $[4,5]$.

New issues and problems arise related to various aspects of this new potential practice; such as practice models of complex genetics, nutrigenetics and pharmacogenetics; clinical utility and validity of genetic tests; and ethical, legal and social aspects. They fall under the area of public health genomics, which is defined as a multidisciplinary field concerned with the effective and responsible translation of genome-based knowledge and technologies to improve population health [7]. Public health genomics uses population-based data on genetic variation and gene-environment interactions to develop evidence-based tools for improving health and preventing disease [8].

One of the most promising implication areas of genomics lies in preventive healthcare, especially for complex diseases. Applications of personalized medicine combined with the advent of health information technology in clinical practice will bring a new kind of medical care: personalized healthcare [9]. It is healthcare that works better for each patient, based partly on scientific information that is new, and partly on technology to make complex information useful [9].

\subsection{Facts \& challenges for the use of genetics in complex diseases}

As we are approaching the end of the decade, science has made important progress in the discovery of genes and polymorphisms. However, their integration into medical practice has been limited. Current examples of the usage of common polymorphisms in clinical practice are polymorphisms in F5 (Factor V), F2 (Factor II) and MTHFRfor thrombophilia 
and perinatology; $A P O E$ for cardiovascular risks and Alzheimer's disease risk; and CYP2C9, CYP2C19 and CYP2D6 for pharmacogenetics. Limited use of polymorphisms in medical practice has been the result of the fact that evidence demonstrating the effectiveness in clinical use is not fully established yet. On the other hand, the current knowledge about the above-mentioned areas already has the potential to be used for the benefit of the individual and society.

There are limitations and room for improvement for the current scientific information. Although the knowledge about associations of polymorphisms with complex diseases is constantly growing, evidence is not fully established. Current linkage analysis and genomewide association studies are focused merely on the genotype-disease relationship. Genotype information can be investigated together with other contributing factors for assessment of disease risks (lifestyle including smoking, nutrition, and exercise; personal health history; family history; environmental exposures, and so on). On the other hand, current studies investigating the interactions between genotype and lifestyle factors are bringing limited evidence owing to small sample sizes. The limited number of prospective studies demonstrates the benefit of selected nutrition or nutritional supplement use based on selected genetic structures [10]. This type of prospective intervention study is needed for various other claims regarding nutrient-genotype interactions.

Limited information suggests that personal risk assessments and personalized recommendations can be a more effective means compared with classical approaches to change the lifestyle of the individuals [11]. In addition, the current level of technology allows us to make personalized risk assessments and develop personalized recommendations based on health information of individuals, including genetic information. There are several initiatives to benefit from genetic data to make personalized risk assessments and recommendations [12-15]. However, the effectiveness of such applications has not been thoroughly evaluated (i.e., controlled prospective studies).

\section{Approach of GENAR Institute}

As the GENAR Institute for Public Health and Genomics Research (Ankara, Turkey), we have been working on the development of a practice model called Gentest ${ }^{\circledR}$, which is an endeavor to face the above-mentioned challenges as an integrative preventive model that utilizes an individual's health information, lifestyle factors, biomarkers and genotype in order to prevent and detect chronic and complex diseases early in a targeted way [16] (for information on GENAR Institute, see Box 1).

The mission of this practice model is changing the behavior and managing the health of individuals according to their health priorities.

The conceptual framework of Gentest is presented in Figure 2. Its components are described in the implementation stages below. 


\section{Box 1: GENAR Institute for Public Health and Genomics Research.}

The GENAR Institute for Public Health and Genomics Research was established in 2004, in Hacettepe University Science Park, Ankara, Turkey. It is the third public health genomics center in Europe and a cooperating institute of Public Health Genomics European Network (PHGEN) [17]. The GENAR Institute aims to transform scientific developments in the area of biotechnology, especially those in genetics and genomics, into products and services that improve human health, quality of life and performance, and extend lifespan. The GENAR Institute has a broad range of working area, mainly on chronic complex diseases. The $R \& D$ activities focus on understanding the underlying genetic and genomic basis of these conditions and developing products based on targeted prevention, early detection and treatment strategies.

The GENAR Institute is comprised of three centers: GENAR Biotechnology and Molecular Genetics Research and Diagnostic Laboratories, which is a high-throughput molecular genetic analysis laboratory; GENAR Center for Nutrigenetics and Lifestyle Research, which focuses on quantifying the nutrition and other lifestyle factors of individuals and developing models for personalized nutrition and lifestyle advice for optimization of individual needs; and the GENAR Center for Personalized Medicine and Pharmacogenetics, which aims to catalyze transfer of developments in genetics to the health of individuals with a public health vision. There has also been an implementation center in Istanbul, Turkey, to pilot the developed models.

Gentest is designed to be implemented in primary-care settings where the health professional(s) (physicians and/or dieticians) can focus on preventive healthcare interventions. For the piloting phase of the model, it is mainly practiced in the Gentest Implementation Center, which is run by GENAR. In addition, health professionals are authorized to be practitioners of Gentest after participating in training of the Turkish Society of Public Health Genomics and Nutrigenetics and the GENAR Institute.

The diseases/health areas that are undertaken in Gentest are selected based on their prevalence, the burden they create and the ability to recommend lifestyle and medical interventions to reduce the risks or delay the onset of these diseases. Currently, the following diseases and health areas are undertaken in Gentest:

- Cardiovascular and cerebrovascular diseases;

- Insulin resistance, Type 2 diabetes and obesity;

- Inflammation;

- Osteoporosis;

- Antioxidation and detoxification mechanisms;

- Cancers in general, and the five most prevalent cancers in particular, which are lung, breast, prostate, colon and stomach. 


\begin{tabular}{|c|c|c|}
\hline Inputs & Assessments & $\begin{array}{c}\text { Optimum Lifestyle and } \\
\text { Medical Plan }\end{array}$ \\
\hline $\begin{array}{l}\text { - Personal information } \\
\text { - Health information } \\
\text { - Living and working } \\
\text { conditions } \\
\text { - Lifestyle information } \\
\text { - Anthropometric and } \\
\text { bioimpedance } \\
\text { measurements } \\
\text { - Biomarkers } \\
\text { - Genotype information }\end{array}$ & $\begin{array}{l}\text { - Nutritional assessment } \\
\text { - Body composition } \\
\text { assessment } \\
\text { - Assessment of physical } \\
\text { activity and exercise } \\
\text { - Assessment of causes of } \\
\text { smoking } \\
\text { - Assessment of current } \\
\text { health information } \\
\text { - Genotype assessment } \\
\text { - Assessment of } \\
\text { biomarkers } \\
\text { - Assessment of current } \\
\text { risks of common complex } \\
\text { diseases }\end{array}$ & $\begin{array}{l}\text { - Medical evaluation } \\
\text { - Medical follow up plan } \\
\text { - Programme for optimum } \\
\text { body composition } \\
\text { - Nutritional } \\
\text { recommendations } \\
\text { - Menu plans } \\
\text { - Exchange options } \\
\text { - Physical activity and } \\
\text { exercise recommendations } \\
\text { - Exercise programmes } \\
\text { - Recommendations on } \\
\text { quitting smoking }\end{array}$ \\
\hline \multicolumn{3}{|c|}{ Follow up plan } \\
\hline \multicolumn{3}{|c|}{$\begin{array}{l}\text { - Weekly, monthly, yearly evaluations } \\
\text { - Revisions of the plans }\end{array}$} \\
\hline
\end{tabular}

Figure 2: Conceptual framework for the Gentest practice model

\subsection{Implementation stages of the practice model}

\subsubsection{Data \& information collection stage}

An individual who applies to the Gentest Implementation Center is first explained what Gentest is, and what it is not (see Table 1). The most suitable Gentest package for that individual's personal characteristics and requirements is chosen with the help of the physician.

Special attention is paid to create proper consumer expectations, as defined in Table 1. For this purpose, a sample Gentest, which is an anonym report of a real case, is presented to the individual. After oral and written acknowledgments explaining the issues summarized in Table 1, the individual signs the consent form for DNA analysis. An appointment is given to come with overnight fasting. 
Table 1: What is Gentest and what is not?

\section{What is Gentest? \\ Gentest is developed to assist health pro- \\ fessional and the individual in their efforts \\ to prevent diseases and promote health. \\ Gentest recommendations are subject to \\ the evaluation of and can be changed by \\ the health professional.}

Gentest can only be implemented through authorized health professionals (physician

and/or dieticians, according to the con-

tent of the packages).

Gentest provides information on disease predispositions and risks.

\section{What is not Gentest?}

Gentest is not a direct to consumer service.

Gentest does not diagnose diseases or give treatment advice.

Gentest does not provide deterministic information if a person will develop a disease or not.

The main purpose of the risk calculations is to provide the lifestyle and medical follow-up interventions in a targeted way. Gentest gives personal recommendations to decrease one's health risks and lead a healthier life within their own genetic Being predisposed to a disease does not mean that the person will definitely develop it. make-up.

Risk calculations are based on population studies and refer to the risk of subgroups of individuals carrying the characteristics of the individual subject to the test. The characteristics used for the calculations are age, gender, health information, biomarkers, lifestyle factors and genetic make-up.

Following the medical follow up and lifestyle recommendations given in Gentest report scientifically reduces one's disease risks.
Carrying the risk of the disease does not mean that the person will definitely develop it. 


\section{Box 2: Inputs of Gentest}

- Personal information

- Age

- Gender

- Health information

- Personal history (current and past diseases and medications)

- Family history

- Living and working conditions

- Occupation

- Past and present occupational and environmental exposures

- Lifestyle information

- Physical activity and exercise

- Smoking and drinking habits

- Supplement consumption

- Nutritional habits

- Food consumption ( $24 \mathrm{~h}$ recall and food frequency)

- Anthropometric and bioimpedance measurements

- Height, body weight and composition

- Waist circumference

- Biomarkers

- Genotype information

When the person comes to the appointment, blood and urine samples are taken. Anthropometric measurements are made using scales and a tape measure, and bioelectrical impedance is used for body composition analysis. Blood pressure and pulse is measured. The individual fills in a detailed questionnaire collecting the necessary inputs listed in Box 2 with the assistance of a health professional. Gentest Food Portion Atlas (Figure 3), which is developed by GENAR, is used during collection of food consumption data. It takes approximately $2 \mathrm{~h}$ for measurements and filling in the information form.

Blood and urine biomarker analysis are carried out by an external biochemical laboratory and the results are forwarded to the GENAR Institute. One tube of blood sample is transported to GENAR Laboratories for genetic analysis.

\subsubsection{Assessments}

The questionnaire is analyzed by the GENAR Center for Nutrigenetics and Lifestyle Research, to quantify the nutrient intake, physical activity status and causes of smoking. The current consumption status of macro- and micro-nutrients are assessed with the analysis of both food consumption records and food frequency questionnaires. 
For each macro- and micro-nutrient, a minimum and maximum level of intake recommendation is determined. These nutrients include: macronutrients such as protein, carbohydrate and fat, including different saturated/unsaturated fats and omega 6/omega 3 fatty acids; vitamins; minerals; and key functional foods. Recommended levels are based on age, gender, current diseases, genetic information and lifestyle information of the individuals. Recommendation algorithms are based on international and national guidelines on macro- and micro-nutrients and literature on nutrition and nutrigenetics research. The recommendations are presented both in tables and figures (for an example of figures, see Figure 4).

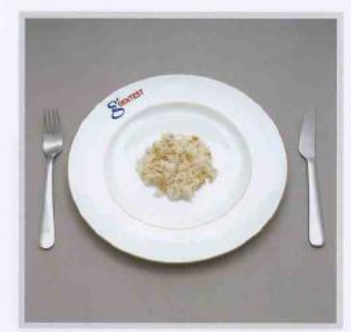

20A: Firing pilan $35 \mathrm{~g}$

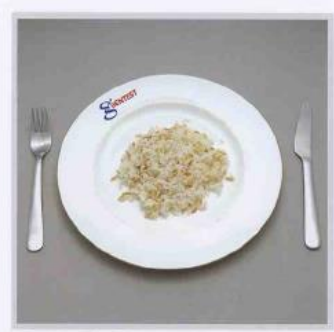

20B: Prinç plav 759

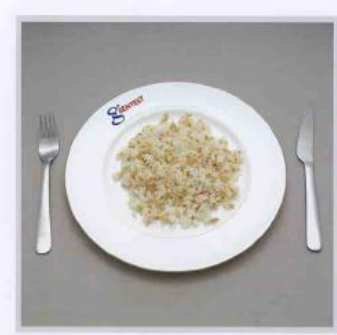

20C: Prinç plav $150 \mathrm{~g}$

Figure 3: An excerpt from the 'Food Portion Atlas'

Vitamin A

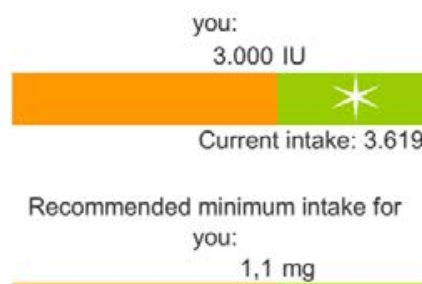

Vitamin B2

(Riboflavin)
Current intake: $1,1 \mathrm{mg}$

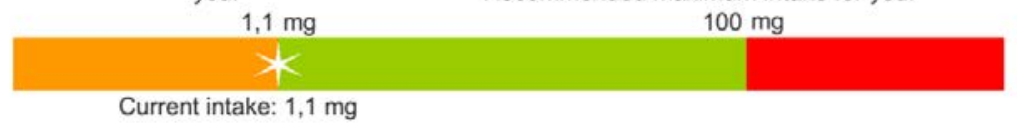

Recommended minimum intake for you:

Recommended maximum intake for you: $200 \mathrm{mg}$

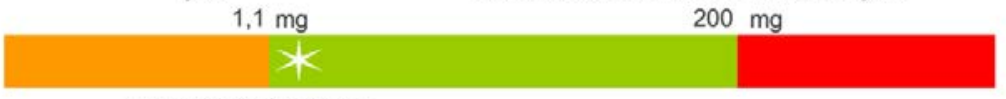

Current intake: $1,6 \mathrm{mg}$

Figure 4: An excerpt from the 'Assessment of Nutrition and Nutritional Supplements' section of a Gentest report

Assessment on body composition is made using anthropometric measurements and bioimpedance analysis (Figure 5). 


\section{Your Body Weight}

\begin{tabular}{|c|c|c|}
\hline $\begin{array}{l}\text { Lower limit } \\
\text { recommended for you: }\end{array}$ & $\begin{array}{l}\text { Upper limit } \\
\text { recommended for you: }\end{array}$ & $\begin{array}{l}\text { Obesity } \\
\text { limit: }\end{array}$ \\
\hline $49 \mathrm{~kg}$ & $66 \mathrm{~kg}$ & $79 \mathrm{~kg}$ \\
\hline
\end{tabular}

\section{Your Body Fat Ratio}

Lower limit

recommended for you:

$\% 23,9$

\section{Upper limit \\ recommended for you:}

$\% 34,9$
Obesity

limit:

$\% 41,0$

Your body fat ratio:\% 43,3

\section{Your Waist Circumference}

Upper limit

recommended for you:

$80 \mathrm{~cm}$

Your waist circumference: $100 \mathrm{~cm}$

Figure 5: An excerpt from the 'Assessment of Body Composition' section of a Gentest report

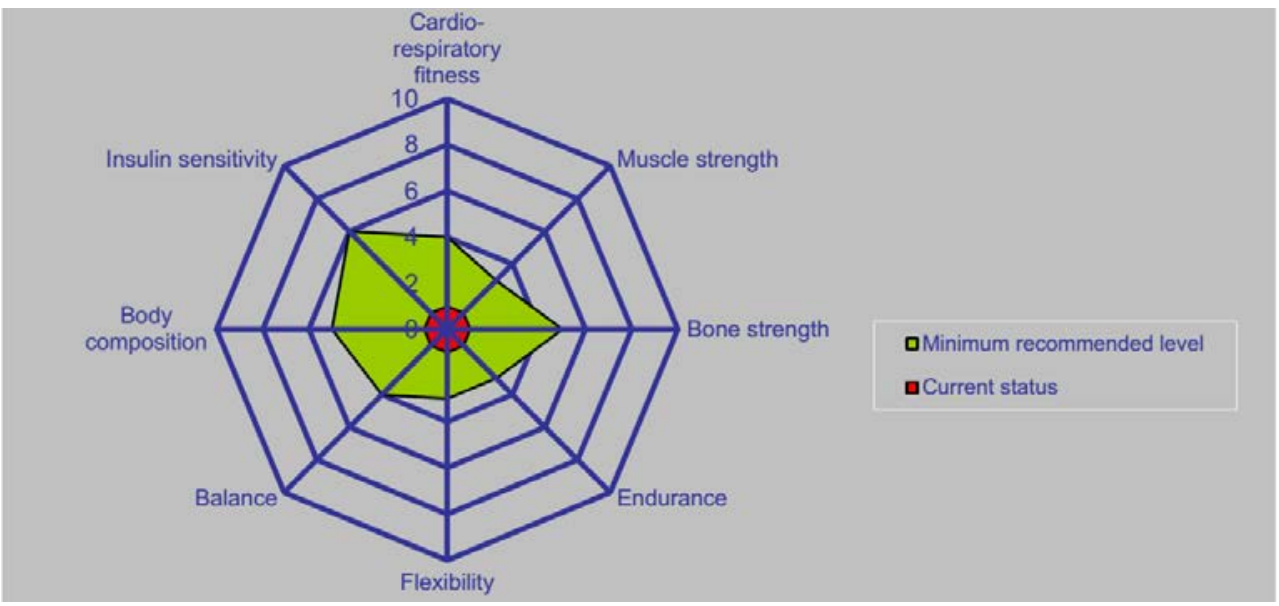

Figure 6: An excerpt from the 'Assessment of Physical Activity and Exercise Status' section of a Gentest report

Current exercise status related to eight different areas of physical fitness is assessed. These areas are cardiorespiratory fitness, muscle strength, bone strength, muscle endur- 
ance, flexibility, balance, insulin sensitivity and body composition. Currently, this assessment is based on the type, intensity, duration and frequency of the exercises carried out by the individual. More objective methods to assess the physical fitness levels of the individual are under development. The recommended level of exercise for each physical fitness area is determined according to disease risks, genetic predispositions and personal preferences (Figure 6).

If the individual is smoking, causes of smoking are also assessed using questionnaires. The types of smoking assessed are nicotine craving/physiological addiction, stimulation, relaxation/pleasure, crutch/tension reduction, habit and handling (hand contact with the cigarette).

In parallel to assessment of the lifestyle information, genetic analysis is carried out by GENAR Biotechnology and Molecular Genetics Research and Diagnostic Laboratories. Genetic analysis covers common polymorphisms related to common complex diseases and conditions listed above (see Approach of GENAR Institute section). For each health area, the results are presented in a table that presents information on the gene, function/role in health-related area, polymorphism and the genotype result of the individual, as well as what the result indicates for that disease/health area (for a sample page, see Figure 7). The number of polymorphisms studied varies from 35 to 65 in the different packages.

\section{CARDIOVASCULAR AND CEREBROVASCULAR HEALTH}

1. sign is used in case that genotype indicates predisposition to that condition, whereas $\mathbf{A}$ sign is used in case of a favorable condition and $<->$ in case of a neutral situation. If not applicable, $\varnothing$ is used. If the predisposition to the condition decreases under some certain life style modifications or this genotype specifically responds well to a certain factor, $\checkmark$ sign in used under "Response to lifestyle modif." column.

\begin{tabular}{|c|c|c|c|c|c|c|c|c|c|c|c|}
\hline \multirow[b]{2}{*}{ Gene } & \multirow[b]{2}{*}{ Full Name } & \multirow[b]{2}{*}{$\begin{array}{c}\text { Function / Role in } \\
\text { Related Health } \\
\text { Area }\end{array}$} & \multirow[b]{2}{*}{$\begin{array}{c}\text { Analyzed } \\
\text { Polymorphism }\end{array}$} & \multirow[b]{2}{*}{ Genotype } & \multirow[b]{2}{*}{$\begin{array}{c}\text { Allele } \\
\text { Structure }\end{array}$} & \multicolumn{2}{|c|}{$\begin{array}{l}\text { Atherosclerosis and } \\
\text { Atherothrombosis }\end{array}$} & \multicolumn{2}{|c|}{ Hypertension } & \multicolumn{2}{|c|}{ Venous Thrombosis } \\
\hline & & & & & & Predisposition & $\begin{array}{c}\text { Response } \\
\text { to lifestyle } \\
\text { modif. }\end{array}$ & Predisposition & $\begin{array}{c}\text { Response to } \\
\text { lifestyle } \\
\text { modif. }\end{array}$ & Predisposition & $\begin{array}{c}\text { Response } \\
\text { to lifestyle } \\
\text { modif. }\end{array}$ \\
\hline APOA1 & $\begin{array}{l}\text { Apolipoprotein } \\
\text { A-I }\end{array}$ & $\begin{array}{l}\text { HDL Structure; } \\
\text { Cholesterol } \\
\text { Metabolism }\end{array}$ & $\mathrm{G}(75) \mathrm{A}$ & Homozygous & AA & $\mathrm{MA}$ & $\sqrt{ }$ & $\varnothing$ & & $\varnothing$ & \\
\hline APOC3 & $\begin{array}{l}\text { Apolipoprotein } \\
\text { C3 }\end{array}$ & $\begin{array}{l}\text { HDL,LDL,VDLD } \\
\text { Structure; } \\
\text { Triglyceride and } \\
\text { Cholesterol } \\
\text { Metabolism }\end{array}$ & C3175G & No variation & CC & $\mathbf{\Delta}$ & & $\varnothing$ & & $\varnothing$ & \\
\hline LPL & Lipoprotein Lipase & Lipid Metabolism & C1595G & No variation & $\mathrm{CC}$ & NA & $\checkmark$ & $\varnothing$ & & $\varnothing$ & \\
\hline LIPC & Hepatic Lipase & HDL Metabolism & $C(514) T$ & Heterozygous & $\mathrm{C} / \mathrm{T}$ & $\varnothing$ & $y$ & $\varnothing$ & & $\varnothing$ & \\
\hline CETP & $\begin{array}{c}\text { Cholesterol Ester } \\
\text { Transfer Protein }\end{array}$ & $\begin{array}{l}\mathrm{HDL} \text { - LDL } \\
\text { Cholesterol } \\
\text { Balance }\end{array}$ & G279A & Heterozygous & G/A & $\Delta$ & $\sqrt{ }$ & $\varnothing$ & & $\varnothing$ & \\
\hline PON1 & Paraoxanase1 & $\begin{array}{l}\text { Lipid Oxidation } \\
\text { Mechanism }\end{array}$ & Gin192Arg & No variation & $G \ln / G \ln$ & $\mathbf{\Delta}$ & & $\varnothing$ & & $\varnothing$ & \\
\hline MMP3 & $\begin{array}{c}\text { Matrix } \\
\text { Metalloproteinase } \\
3\end{array}$ & $\begin{array}{c}\text { Matrix Deposition; } \\
\text { Atherosclerotic } \\
\text { Mechanism }\end{array}$ & $5 A \sqrt{ } 6 \mathrm{~A}$ & No variation & $5 \mathrm{~A} / 5 \mathrm{~A}$ & $\mathbf{\Delta}$ & $\sqrt{ }$ & $\varnothing$ & & $\varnothing$ & \\
\hline IL-6 & Interleukin-6 & $\begin{array}{l}\text { Inflammatory } \\
\text { Response }\end{array}$ & $G(-174) C$ & Heterozygous & $\mathrm{G} / \mathrm{C}$ & $\mathrm{NA}$ & $\sqrt{ }$ & $\varnothing$ & & $\varnothing$ & \\
\hline TNF- $\alpha$ & $\begin{array}{l}\text { Tumor Necrosis } \\
\text { Factor Alpha }\end{array}$ & $\begin{array}{l}\text { Inflammatory } \\
\text { Response }\end{array}$ & $\mathrm{G}(-308) \mathrm{A}$ & No variation & GG & $\mathbf{A}$ & & $\varnothing$ & & $\varnothing$ & \\
\hline
\end{tabular}

Figure 7: An excerpt from the 'DNA Analysis Results and Genotype Assessment Table' section of a Gentest report 
Polymorphisms in the health area of interest are selected qualitatively based on considering a number of aspects. The studies that show a positive association and no association are evaluated in their design, statistical power, presented odds ratios and $p$-values, as well as the credibility of the published journal. Frequency of the polymorphism in white Caucasians are also taken into account. Studies on gene-lifestyle interactions are also evaluated with this perspective in mind.

Biochemical markers such as blood lipids, fasting plasma glucose, liver and kidney function tests, homocysteine, high-sensitivity C-reactive protein and fibrinogen are analyzed by external clinical laboratories. The biomarkers are selected and assessment methods are developed in light of the current medical guidelines and literature.

The results of lifestyle assessment, genetic analysis and biomarker tests are gathered in the GENAR Center for Personalized Medicine and Pharmacogenetics, in order to produce the report of the individuals.

In the GENAR Center for Personalized Medicine and Pharmacogenetics, risk assessments for the most common chronic complex diseases are made. These include myocardial infarction, stroke, Type 2 diabetes, osteoporosis and the five most common cancers (lung, breast, prostate, colon and stomach). Risk assessment algorithms are based on risk factors conveyed by various epidemiological studies and risk assessment models. The genetic analysis results are also used as a factor in risk assessment, but with very small effect sizes given the limited demonstrated effect of discovered polymorphisms on overall risks of diseases. The inputs of the risk assessment algorithms are listed in Box 2.

Risks are presented for two cases: the estimated risk using the current data and the estimated risk for the case that the recommended optimum lifestyle and medical follow-up plan is followed (for examples of risk graphics, see Figures $8,9 \& 10$ ).

Risk graphics (both the current and estimated reduced risk with the optimum lifestyle and medical follow-up plan) are presented in the report for two main purposes. The first one is to provide the lifestyle and medical follow-up interventions in a targeted way. The second one is to promote lifestyle changes through creating personal vulnerability perception and individual risk perception [18]. 


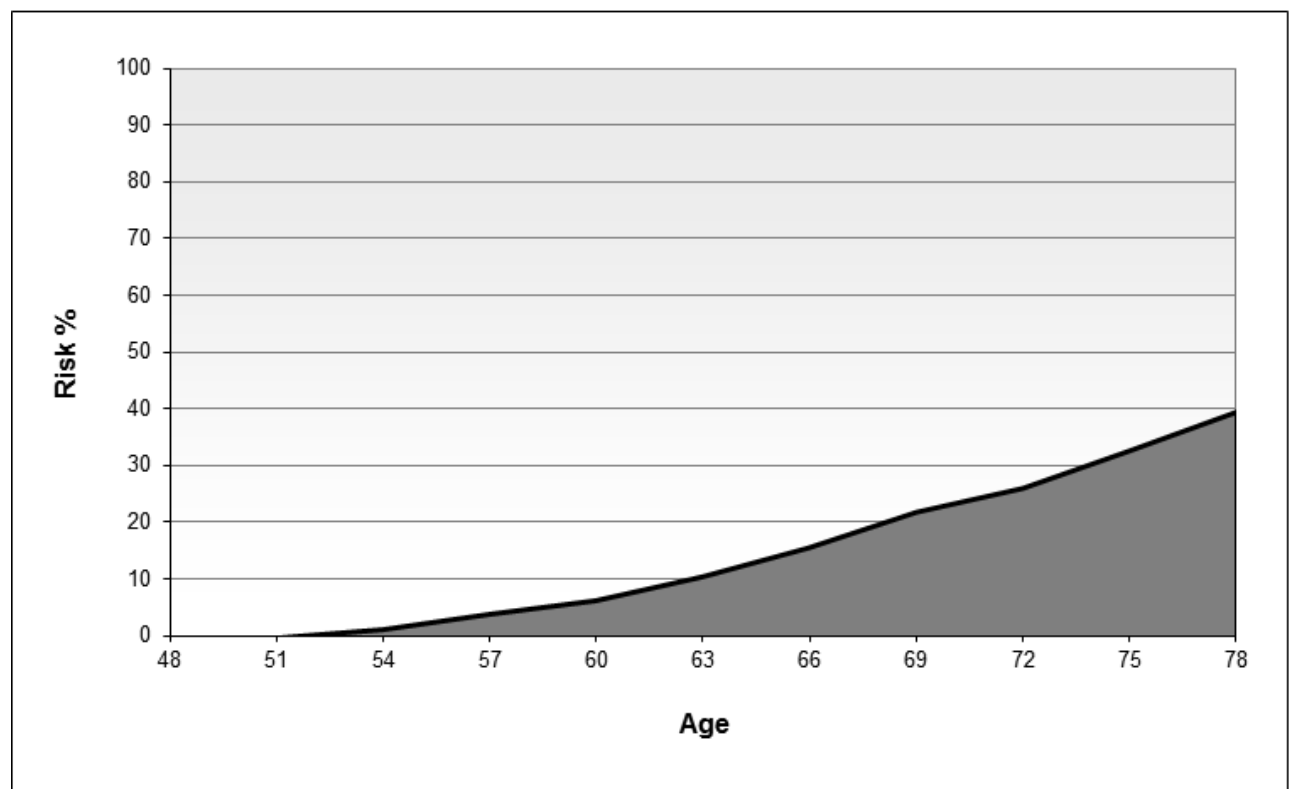

Figure 8: An excerpt from the 'Disease Risks' section of a Gentest report: 'Heart Attack Risk with Current Lifestyle in the Following 30 Years'

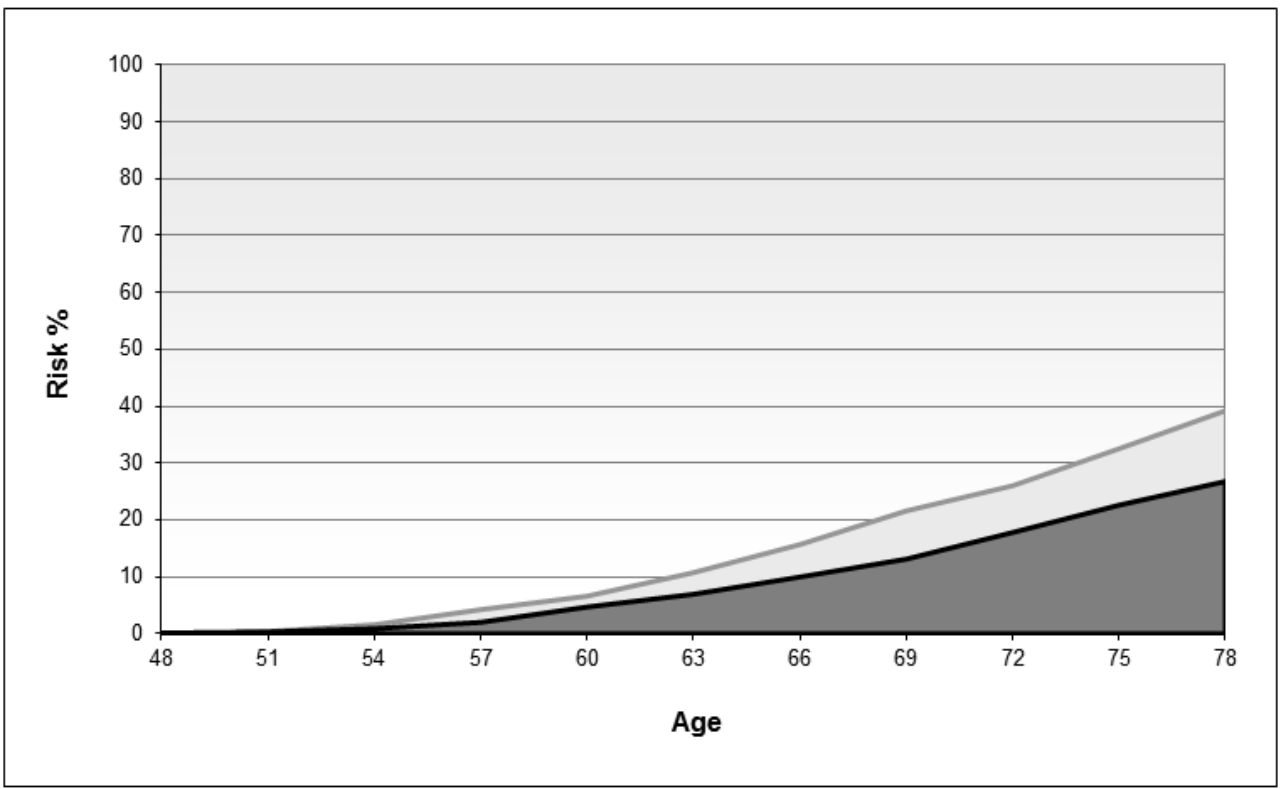

Figure 9: An excerpt from the 'Disease Risks' section of a Gentest report: 'Reduction of Heart Attack Risk in the Following 30 Years After Implementation of Optimum Lifestyle and Medical Follow-up Plan' 
Your Colon Cancer Risk: Above Average

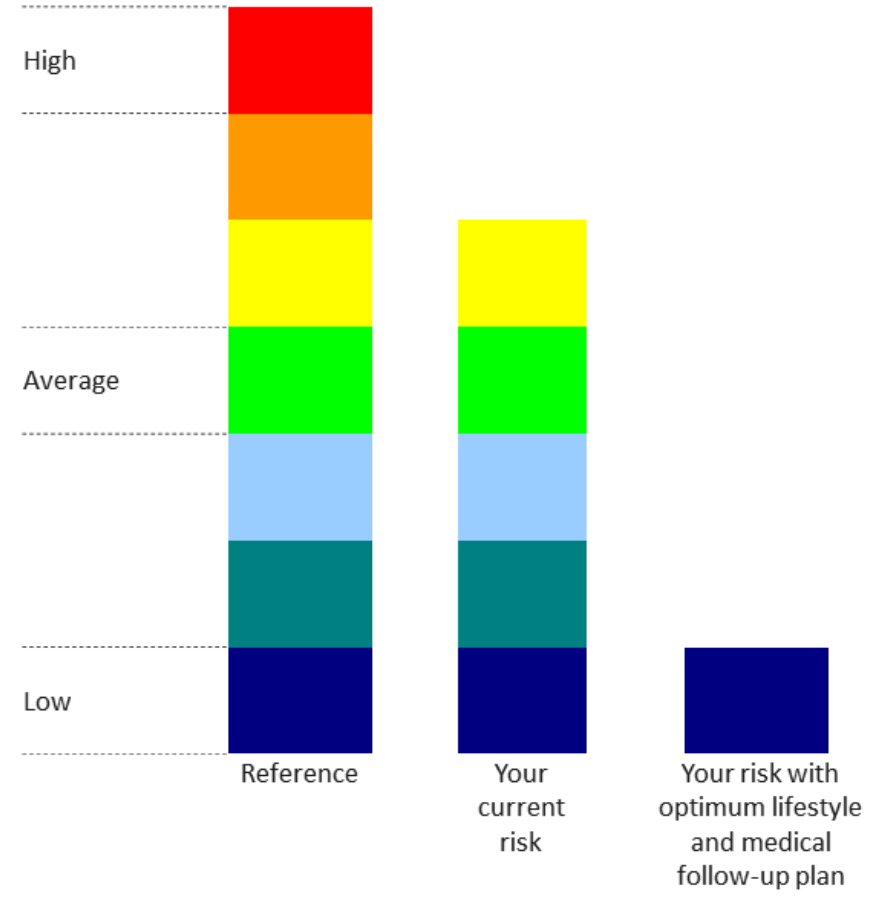

Figure 10: An excerpt from 'Disease Risks' section of a Gentest report: 'Colon Cancer Risk'

The report of an individual includes the results of the above-mentioned assessments and an 'Optimum Lifestyle Plan' developed for the individual. It includes a plan for reaching and/or maintaining optimum body composition, personal nutrition plans and food exchange lists, supplement plans and exercise plans. Smoking cessation recommendations are personalized according to the causes of smoking. Recommendations are given for medical follow-up with personalized screening plans. For all medical follow-up recommendations, it is highlighted that they are subject to evaluation of the physician and can be changed if necessary. If any medical problem that needs further investigation or curative interventions is detected, it is presented in the 'physician note', which is provided for the physician separately.

When developing methodologies for all components of Gentest, the most important decision criterion is this: for all the information and recommendations we give, there has to be good probability of benefit with no possibility of harm. This is ensured by always complying with the nutritional and medical guidelines. For example, recommendations on the upper and lower limits of the intake of a nutrient are always within the upper and lower limits that are recommended in the nutrition guidelines. Properties that can be used to 
further personalize the recommendations, but not referred in the guidelines yet, such as genetic information, are used to narrow down the general recommendation range.

\subsubsection{Counseling \& follow up}

Gentest reports are ready approximately 4-8 weeks after sample taking and filling out the questionnaire. The report is sent out to the physician who explains the report results to the individual during an hour long appointment. The nutrition program is explained by a dietician trained in nutrigenetics.

If the individual would like to have assistance in making the lifestyle changes, the followup program is started. This option is usually chosen by the overweight or obese individuals who are recommended to reach their optimum body composition.

\subsection{Experiences of GENAR}

The practice model has been in service for piloting purposes for approximately 2 years in Turkey. Approximately 500 individuals have used this pilot service. The results of this pilot implementation phase are being assessed and will be published in another manuscript. A noteworthy observation is that Gentest might have a better outcome for behavior change than providing general information on healthy behaviors. In particular, initiation of exercise, smoking cessation and weight loss has been observed. This observation surely needs to be confirmed with publication of related data.

We think that Gentest may have an important effect in creating awareness by informing individuals about their current lifestyle and genetic predispositions. Furthermore, it causes an attitude change by creating a vulnerability perception. Finally, it is observed that behavior change is achieved with the follow-up program and the trainings (Figure 11).

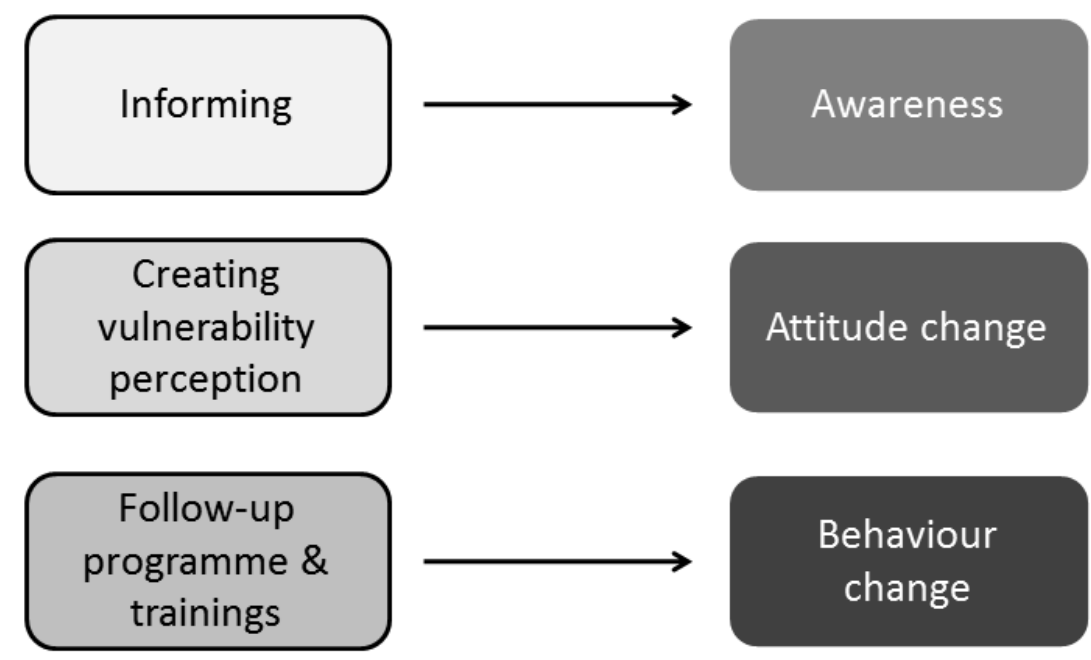

Figure 11: M odel of attitude and behavior change with Gentest 


\section{Criticisms to the approach of the GENAR Institute}

Our practice model has been presented to the scientific community at a number of occasions. These are the 1st Congress of the International Society of Nutrigenetics/Nutrigenomics (Athens, Greece, 13 November, 2007) [16], 16th European Conference on Public Health, European Public Health Association (Lisbon, Portugal, 7 November, 2008), Conference on Public Health Genomics in Europe, Public Health Genomics European Network (Istanbul, Turkey, 26 November, 2008). The practice model being comprehensive (containing several factors such as personal, medical and lifestyle information, and genetic information), multidisciplinary, prevention-orientated and implemented through health professionals have been the appraised characteristics.

On the other hand, the main criticism to this practice model has been that its effectiveness has not been demonstrated yet. Since 2004, GENAR has developed the model, piloted its components and the whole model, and further developed it with feedback. This gave GENAR the chance to see if such a model is applicable in a preventive healthcare setting, and if there is a potential benefit for the consumers. Currently, we are working on the design and implementation of research for the evaluation of the effectiveness of the proposed practice model. The effectiveness will be assessed from outcome perspective (prevention of diseases and disease complications), bioprocesses perspective (lipid profile, inflammation, glycemic control and so on), lifestyle changes and applicability. Collaboration with cohorts are also planned to further develop the algorithms.

\section{Conclusion}

Public health genomics and personalized healthcare will play major role in combating the chronic complex disease burden of the aging populations of the $21^{\text {st }}$ century. In parallel to exponentially increasing knowledge gained through research, healthcare systems need to foresee these upcoming developments and prepare for the transition. The approach of GENAR is an example of the translation of genome-based knowledge into preventive healthcare. The definition of public health genomics suggests that this translation should be effective and responsible [8]. By assuring that the information and the recommendations has good probability of benefit but has no possibility of harm, the Gentest practice model can be considered as a responsible one. However, the effectiveness has not yet been demonstrated.

\section{Future perspective}

As the populations continue to age, the burden created by complex diseases will increase. The future healthcare systems will not be able to cope with the societal and economic burden of complex diseases. An effective intervention model including lifestyle changes for prevention of these diseases is urgently needed. Like we did a century ago with the vaccines against infectious diseases, the world needs a cost-effective health intervention 
to prevent complex diseases, as well as decrease the disability and increase the life quality of aging populations.

In order to achieve this goal, the medicine of the future needs to target the individual, rather than general public or population subgroups. Thus, healthcare practice models need to be individualized, assessing different characteristics of the individuals, such as personal health data, lifestyle information and genetic information, to provide individualized interventions and recommendations. This vision is defined as 'personalized healthcare'.

Personalized healthcare holds great potential to combat the burden of complex diseases. In 5-10 years' time, personalized healthcare interventions are expected to be widely utilized in primary care settings for primary and secondary prevention of complex diseases and their complications.

\section{Executive summary}

\section{The need for effective intervention models to combat complex diseases}

- Complex diseases, which stem mainly from the complex interaction of the human genome with lifestyle and environmental factors, cause the main burden of disease in the $21^{\text {st }}$ century. Currently, the best-known prevention for complex diseases is adopting a healthy lifestyle. However, this is not achieved in many places of the world. Effective intervention models including lifestyle changes for prevention of these diseases is urgently needed.

\section{Facts \& challenges for the use of genetics in complex diseases}

- In the late 1990s and early 2000s, it was foreseen that genetics/genomics would revolutionize medicine, and genetics would become a tool widely used for prediction, diagnosis and to optimize treatment in most common diseases within the current decade. As we are approaching the end of the decade, science has made important progress to discover genes and polymorphisms. However, their integration to medical practice has been limited, owing to the fact that evidence demonstrating the effectiveness of the genomic markers in clinical use is not fully established yet. On the other hand, the current knowledge about the abovementioned areas already has the potential to be used for the benefit of the individual and society.

\section{Approach of the GENAR Institute}

- The GENAR Institute for Public Health and Genomics Research has developed a practice model called Gentest ${ }^{\circledR}$ as an integrative preventive model which utilizes individual's health information, lifestyle factors, biomarkers and genotype in order to prevent and early detect chronic and complex diseases in a targeted way. 
Based on the results of the aforementioned components, an optimum lifestyle plan is developed, including personal menu plans and exchange lists, exercise plans, smoking cessation recommendations based on the individual causes of smoking, and a medical follow-up plan.

- The mission of this practice model is changing the behavior and managing the health of individuals according to their health priorities. It is thought that the model creates awareness by informing individuals about their current lifestyle and genetic predispositions. Furthermore, it causes an attitude change by creating a vulnerability perception. Finally, it is observed that behavior change is achieved with the follow-up program and the trainings.

\section{Criticisms to the approach of GENAR Institute}

- The practice model being comprehensive (containing several factors of the individual), multidisciplinary, prevention orientated and implemented through health professionals have been the appraised characteristics. On the other hand, a critical point is raised, which is that the effectiveness of the intervention is not yet demonstrated.

- The efforts of GENAR so far has been on the development of the model and piloting its components and the whole model. The crucial future step is to conduct a research for evaluation of the effectiveness of the proposed practice model.

\section{Conclusion}

- Personalized healthcare holds a great potential to combat the burden of complex diseases, provided that the safety is ensured and the effectiveness of the utilized tests and practice models are demonstrated scientifically.

\section{Acknowledgements}

We would like to acknowledge and thank for the leadership of Dr. Serdar Savas who founded and has been leading the GENAR Institute for Public Health and Genomics Research, making it possible for GENAR to develop Gentest $₫$ and for his great contribution to the development of the model and its components.

We would like to acknowledge and thank for valuable scientific and conceptual contribution of Dr. Keith Grimaldi in the early phases of the development of Gentest. We would like to thank all the past and present personnel of the GENAR Institute for their contributions to development and pilot implementation of Gentest. We would like to thank Marco J. de Langen for his careful review and feedback on the article. 


\section{Financial \& competing interests disclosure}

Tomris Cesuroglu, Sefayet Karaca and Sema Erge are employed by GENAR Institute for Public Health and Genomics Research and worked on the development of the Gentest model. The GENAR Institute for Public Health and Genomics Research is a private institute founded by B. Serdar Savas. Some of the components of Gentest are developed as a part of the OBESIS project (Development of a Genetic and Information Technologies Assisted System on Obesity Treatment and Control), which is partially funded by Technology and Innovation Funding Programs Directorate (TEYDEB) of the Scientific and Technological Research Council of Turkey (TUBITAK) (project no: 3060480). The authors have no other relevant affiliations or financial involvement with any organization or entity with a financial interest in or financial conflict with the subject matter or materials discussed in the manuscript apart from those disclosed.

\section{References}

1. Omran AR. The Epidemiologic Transition: A Theory of the Epidemiology of Population Change. Milbank Q. 2005, first published 1971;83(4):731-57. The Milbank Memorial Fund Quarterly, Vol. 49, No. 4, Pt. 1, 1971 (pp. 509-38).

2. Projections of Mortality and Burden of Disease: World Health Organization; 2006 [1 November 2008]. Available from: http://www.who.int/healthinfo/global_burden_disease/projections2002/en/.

3. Simopoulos AP. Genetic Variation: Nutritional Implications in Nutrigenetics and Nutrigenomics. World Rev Nutr Diet. 2004;93:1-28.

4. Bell J. The new genetics in clinical practice1998 1998-02-14 08:00:00. 618-20 p.

5. van Ommen GJB, Bakker $E$, den Dunnen JT. The human genome project and the future of diagnostics, treatment, and prevention. The Lancet.354:S5-S10.

6. Collins FS, McKusick VA. Implications of the human genome project for medical science. Jama. 2001;285(5):540-4.

7. Genome-based Research and Population Health. Report of an expert workshop held at the Rockefeller Foundation Study and Conference Centre Bellagio, Italy, 14-20 April 2005: Public Health Genetics Unit, Cambridge, UK; Institute for Public Health Genetics, University of Washington, Seattle, USA; Office of Genomics \& Disease Prevention, Center for Disease Control and Prevention, Atlanta, USA 2005. Available from: http://www.phgen.eu/typo3/fileadmin/downloads/bellagio011807.pdf.

8. Centers for Disease Control and Prevention - National Office of Public Health Genomics. 10 years of public health genomics at CDC 1997-2007. Atlanta, GA: Centers for Disease Control and Prevention; 2007. Available from: www.cdc.gov/genomics/about/reports/2007/index.htm.

9. United States Department of Health and Human Services. Personalized Health Care: Opportunities, Pathways, Resources 2007. Available from: http:// www.genome.gov/Pages/Policy/PersonalizedM edicine/DHHSReportPersonalizedHealth. pdf.

10. Kornman K, Rogus J, Roh-Schmidt H, Krempin D, Davies AJ, Grann K, et al. Interleukin-1 genotype-selective inhibition of inflammatory mediators by a botanical: a nutrigenetics proof of concept. Nutrition.23(11):844-52. 
11. Arkadianos I, Valdes AM, Marinos E, Florou A, Gill RD, Grimaldi KA. Improved weight management using genetic information to personalize a calorie controlled diet. Nutrition journal. 2007;6:29.

12. 23andM e [July 2009]. Available from: www.23andme.com.

13. Navigenics [July 2009]. Available from: www.navigenics.com.

14. deCODEme deCODE Genetics; [July 2009]. Available from: www.decodeme.com.

15. EUROGENE: eTEN Project [28 August 2009]. Available from: http://eurogene.biomed.ntua.gr/.

16. Savas B. A Case in Commercial Applications of Nutrigenetics. Abstract A31. In: 1st Congress of the International Society of Nutrigenetics/Nutrigenomics (ISNN). J Nutrigenet Nutrigenomics. 2007;1(1-2):72.

17. Public Health Genomics European Network [August 2009]. Available from: http://www.phgen.eu/.

18. Renner B, Schupp H, Vollmann M, Hartung F-M, Schmälzle R, Panzer M. Risk perception, risk communication and health behavior change. Zeitschrift für Gesundheitspsychologie. 2008;16(3):150-3. 
PART III

HOW TO INTEGRATE? 
A camel is a horse designed by a committee.

Attributed to Sir Alec Issigonis 


\section{Chapter 7}

\section{A systematic approach to assess integration of a preventive personalized health care model}

into health systems

Tomris Cesuroglu, Elena Syurina, Anja Krumeich, Frans Feron and Serdar Savas

A systematic approach to integration of a preventive personalized health care model into health systems. Submitted. 


\begin{abstract}
It is a serious bottleneck to integrate 'personalized medicine/personalized health care' into health systems. To solve this, we propose applying a systematic approach to identify and assess issues related to integration. The presented case was developed on a preventive personalized health care model, but it can be applied to other practice models in different countries.
\end{abstract}


Recent developments in science and technology will allow us to shift from general recommendations and "one size fits all" strategies towards more personalized interventions throughout the whole spectrum of health care, from prevention to therapy. This shift is driven by advances in 'genome-based knowledge and technologies' and 'information and communication technologies' (ICT), as well as by the general societal trend towards 'personalization'. Although this vision is shared by various opinion leaders and institutions, the terms they use for it and their definitions of what it entails differ considerably. Nevertheless, most definitions seem to agree on the attributes of it being Predictive, Personalized, Preemptive/ Preventive, and Participatory, previously coined as the term 'P4 Medicine' by Leroy Hood $[1,2]$.

Among the variety of terms, personalized health care seems to be the most comprehensive [3] in terms of scope and depth [4] (see also Chapter 4). The term 'health care' covers not only medicine, i.e. diagnosis and therapy, but the whole spectrum of health related interventions [4]. Personalized health care utilizes, but is not restricted to genomic medicine and other technology driven personalized medicine tools [5]. Any advancement that serves to better prevention, prediction and personalization can be part of personalized health care. Most importantly, personalized health care provides a holistic approach to the individual, covering his/her different characteristics such as personal health history, family health history, lifestyle, genomics, other biomarkers, and socioeconomic factors, rather than focusing on a single determinant of health [4]. Thus, personalized health care offers a valuable opportunity to develop and deploy personalized interventions for prevention of chronic complex diseases, which create a serious burden on health systems and societies in the $21^{\text {st }}$ century $[6,7]$.

Regardless the struggle for common terminology, one problem is common across the fields: integration of 'personalized' practices into different health systems. Personalization of medicine and health care has been on the agenda of the scientific community for over a decade. Nevertheless, integration of related technologies into the health system for the benefit of the population has been slower than initially expected. To tackle this problem effectively, we need to have a holistic view of the issues to be thought of and covered. As all the topics within health systems are interrelated, this approach can be more effective than listing items individually and trying to solve them one by one, independent from each other and the general context. We must first see the broader picture.

This paper describes our attempts to obtain an overview of all issues (and their interconnections) involved to when integrating a preventive personalized health care model into health systems, developed particularly for the European context. First, we will briefly introduce the practice model for prevention of chronic diseases we used as an example and justify the need for a systematic approach for its integration. Upon introducing the overarching framework that guided our efforts, we'll explain how the systematic approach was developed and applied to identify issues around integration of the practice model in Euro- 
pean context. Then, possible uses and potential benefits of such a systematic approach will be summarized. Concluding remarks will call to focus on health rather than disease, and to establish a dialog between the fields of 'personalized medicine/personalized health care' and health systems and policies.

\section{Integration of a preventive personalized health care model}

A personalized health care model called Gentest was developed in Turkey for prevention of chronic complex diseases in primary care services [6](for more information, see Box 1). After its pilot, it was decided to look for possibilities for further expansion of the practice, targeting other European countries.

\section{Box 1: A practice model in personalized health care}

The practice model we used, Gentest, was developed by GENAR Institute for Public Health and Genomics Research in Turkey as an endeavor to face the challenge of chronic complex diseases [6]. It utilizes individuals' personal health information (clinical information including family history), detailed lifestyle analysis (nutrition, exercise and smoking), body composition, genotype, and conventional biomarkers (such as LDL cholesterol, fasting plasma glucose, etc.) in order to prevent development and progression of chronic diseases in a targeted way. Based on the analysis of the aforementioned components, risk assessments for the major chronic diseases are compiled. The range of conditions includes heart attack, stroke, type 2 diabetes, common cancers (lung, breast, prostate, colon, and gastric cancers), and osteoporosis. Diseases selected for the model represent the highest burden of diseases in Turkey. Using all assessments, an optimum lifestyle plan, including personal menu plans and food exchange lists, exercise plans, smoking cessation recommendations, and a medical follow-up plan are drawn. The model is implemented via health care professionals, in particular physicians. Its mission is to change the behavior and manage the health of individuals according to their health needs and priorities.

The purpose of Gentest model is prevention of the major chronic diseases and their complications, within the whole continuum of their development and progression, i.e. from the point where there is no disease to the point where there is fully established disease and further towards its complications. The results of the analysis and recommendations change depending on where a specific individual is on the disease development and progression line for different conditions. The general aim is to catch the individuals on as early as possible stages of disease development to slow down, delay, or if possible, reverse the progression towards the disease and its complications. For this purpose, the model aims to include as much as possible of all determinants of health to make a 360 degree assessment of health of the individual. 
Gentest was developed by GENAR Institute for Public Health and Genomics Research, which was established in 2004, in Hacettepe University Science Park, Ankara Turkey. It was identified as a best practice model for public health genomics in Europe by the Public Health Genomics European Network (PHGEN). It was piloted on more than 500 individuals in Turkey via authorized practitioners (mainly physicians) and now offered as a privately paid health service via authorized practitioners (physicians) in major cities in Turkey.

Based on the experiences with Gentest, feedback from scientific and industrial communities, and current trends and developments in the world, it was agreed that the main implementation area for such a preventive intervention would be primary care services in Europe, where it can be provided via 'general practitioners/family physicians' (will be collectively abbreviated as ' $G P^{\prime}$ '). However, there has been no example of such a preventive personalized health care model provided to large populations. Thus we were faced with questions like "What are the issues we need to look at when investigating the potential integration of Gentest into primary care services? And how can we identify them?"

To answer them, we first looked at the scientific and grey literature on 'personalized medicine/personalized health care'. We identified that the main focus in this field is on scientific and technological developments, in particular on diagnosis and therapy. Issues related to integration into health systems are mainly tackled in a piecemeal approach with bulletpoint lists on 'challenges' or 'barriers'. The topics raised usually concentrate around licensing/approval by authorities, reimbursement/finance, data use and protection, physician and patient acceptance/education, and ethical, legal and social implications [2, 8-14]. (See also Chapter 4) These indeed are important issues that need to be tackled; however it is arguable if they make a whole picture when they come together and whether their coverage will automatically improve the integration process. M oreover, these points are mostly identified for diagnostics and therapeutics scenarios, and have limited relevance to preventive models to be integrated into primary care services. In order to avoid this fragmented vision, there is a need for a systematic approach to assess how a 'personalized' practice can be integrated into a country's health system.

\section{How to analyze integration into health systems}

In search for a systematic approach, we turned to the field of health systems and policies to see what analytical approaches to health systems exist. Among the various conceptual frameworks of health systems [15-19], the one developed for World Health Organization's health system performance assessment efforts in 2000 [16] provides a comprehensive way of looking at the a health system. We used an adapted and elaborated version of this model (see Chapter 2), where a health system has four key functions: stewardship, financing, health services and resource generation (the elaboration was done in particular under 'health services'). These functions provided the overarching framework of our systematic approach (Table 1). 
Table 1: Functions of a health system

\section{A. Stewardship}

○ Priority setting

- Legislation and regulation

- Steering

B. Financing

- Administration and management

- Revenue collection

- Fund pooling

- Purchasing

C. Health services (listed in order from societal to individual and general to specialized)

- Environmental health services

- Population based health protection

- Population based health promotion

- Population based disease prevention

- Personal health promotion services

- Personal disease prevention services

- Primary diagnostic and curative services

- Primary care rehabilitative services

- Specialized care

\section{Resource generation}
- Human resources
- Physical resources
- Knowledge

Source: Adapted and elaborated version of Murray and Frenk [16] by Savas and Cesuroglu (Chapter 2)

By using this framework (Table 1) and our preventive personalized health care model (Box 1), we carried out an exercise to identify which areas need to be assessed when investigating how this practice model could be integrated into health systems in Europe. We identified 24 areas that need to be assessed under the functions and sub-functions of health systems (the first column in Table 2).

Not all of the 24 areas and numerous criteria and issues have the same importance when a practice model is integrated into a health system. Therefore, we organized the areas in three levels of importance: crucial, priority and secondary (the second column in Table 2). Crucial areas are a 'must' and should be the first areas to be investigated in a given country. If they don't yield favorable results, it is virtually impossible for the practice model to be integrated in that country and therefore, investigation of other areas would not be necessary. For example, due to the economic crisis, some countries in Europe are facing 
drastic budget cuts in public sector (Area 13 in Table 2 and Table S1 in supplementary material). In those countries where austerity policies are implemented, it is practically impossible to make a new investment such as the practice model.

Another crucial area we identified is ' 15 . Strength and place of primary care services in the health system and its relationship with other levels of care'. The beneficial effects of the practice model would be most prominent in systems where the primary care physician (GP) has the function to manage the health and risks of individuals registered to his/her practice. If the primary care services are strong and the GPs have a 'gate keeper' position regulating access to specialized care, implementation of the practice model would yield the best results. If it doesn't have the gate-keeper function, it will be very difficult for primary care settings to assume the function of managing the health and risks of individuals and, therefore, application of the practice model wouldn't yield expected benefits. Therefore, we identified that a strong primary care service structure is one of the prerequisites for implementation of this model.

Priority areas are the ones that should be 'favorable' in a given country. If assessment of a priority area doesn't yield a favorable profile, it will be difficult to integrate the practice model as envisaged in that country, even if it fits in the other areas perfectly. For example, if 'the general environment and culture of the health system' (Area 9) is not favorable for innovations, it would be very difficult to integrate the practice model.

Secondary areas are the 'complementary' ones that need to be addressed to prepare and plan for the investigation of the practice model.

In order to complete the exercise, we used examples from the Netherlands and the UK to check if and how our areas were applicable in real systems, as well as to identify new issues as necessary. We chose these two countries since we had access to information on them. In order to enrich the exercise, we used relevant examples from other European contexts as we came across with them. An example of an issue identified on a country level is a specific Dutch regulation covering 'screening' programs, which has implications for preventive health interventions. Although Gentest is not intended to be a 'screening' tool, due to various laboratory tests used, it may fall under this law (Area 3. Regulation of non-diagnostic services which aim prevention, Table S1). Thus this systemic approach helped us to identify that if this practice is integrated into primary care services in the Netherlands, it should be made clear if and what parts of it fall under this regulation. Moreover, similar regulations should be screened for in other candidate countries as well. Another prominent example is the UK's recent initiatives on using whole genome sequencing (WGS) for 'research' purposes for large number of patients (100.000), which may be considered as a pilot for the possible future services of WGS [20]. This can be a signal of a 'pro-genomics' environment in this country and facilitate the integration (Area 9. General environment and culture of the health system for innovation, Table S1). 
Table 2: Areas identified for assessment of a country's health system for integration of the practice model

\begin{tabular}{|l|l|}
\hline AREAS & IMPORTANCE \\
\hline A. STEWARDSHIP & \\
\hline Prionity setting & \\
\hline 1. Health policies, strategies and programs & Secondary \\
\hline 2. Strategies on relevant applications and technologies & Priority \\
\hline Legislation and regulations & \\
\hline 3. Regulation of non-diagnostic services which aim prevention & Secondary \\
\hline $\begin{array}{l}\text { 4. Regulation of genetic testing, including predictive testing for im- } \\
\text { plementation in primary care services }\end{array}$ & Priority \\
\hline 5. Regulations on ethical issues in terms of implementation & Secondary \\
\hline 6. Regulations on liability of the practice model & Secondary \\
\hline 7. Regulations on data protection, privacy and confidentiality & Secondary \\
\hline 8. Regulations on cross-border health care & Secondary \\
\hline Steering & \\
\hline $\begin{array}{l}\text { 9. General environment and culture of the health system for innova- } \\
\text { tion }\end{array}$ & Priority \\
\hline $\begin{array}{l}\text { 10. Stakeholders, processes and mechanisms involved in implementa- } \\
\text { tion of new applications in health care }\end{array}$ & Secondary \\
\hline Administration and management & \\
\hline $\begin{array}{l}\text { 11. Mechanisms related to evaluation of new applications and deci- } \\
\text { sion making in health care services }\end{array}$ & Priority \\
\hline B. FINANCING ${ }^{2}$ & \\
\hline 12. Health financing system of the country & Priority \\
\hline 13. General health expenditure structure of the country & Crucial ${ }^{3}$ \\
\hline 14. Payment mechanisms for primary care services, i.e. GPs \\
\hline C. HEALTH SERVICES (Primary care services) & Prucial \\
\hline $\begin{array}{l}\text { 15. Strength and place of primary care services in the health system } \\
\text { and its relationship with other levels of care }\end{array}$ & Crucial \\
\hline 16. Scope of primary care in terms of (primary) prevention & Secondary \\
\hline 17. Performance assessment in primary care services & Secondary \\
\hline 18. Service delivery in primary care services & \\
\hline
\end{tabular}




\section{AREAS}

IM PORTANCE $^{1}$

D. RESOURCE GENERATION

\begin{tabular}{l}
\hline Health workforce \\
\hline 19. Health workforce in primary care services \\
\hline 20. General environment and culture of the primary care services for \\
implementation of a new practice model
\end{tabular}

Knowledge

21. Education and training of the health workforce

22. Information flow and information systems

\begin{tabular}{|l|l|}
\hline Physical resources & \\
\hline 23. Logistical aspects & Secondary \\
\hline 24. Laboratory services & Secondary \\
\hline
\end{tabular}

Notes of the table:

${ }^{1}$ Importance in terms of the decision for integration of the practice model in the given country

${ }^{2}$ Within the general framework of functions of health systems (Table 1), financing has three sub-functions: revenue collection, fund pooling, purchasing. However, for the purpose of assessment of integration a practice model, those subdivisions were not necessary. Therefore, areas are identified for financing function in general.

${ }^{3}$ Under 'normal' circumstances, this area wouldn't be labeled as 'crucial'. However, due to 'extraordinary' economic situation in Europe, i.e. the economic crises, some countries in Europe are implementing very strict monetary policies and are having budget cuts for even regular health services. For those countries, it might be almost impossible to consider making a new investment in health care, such as the practice model. Therefore, this area needs to be investigated before moving to the other areas. See further explanation under '13. General health expenditure structure of the country' in Table $\mathbf{S} 1$ in Supplementary material.

${ }^{4}$ Within the general framework of functions of health systems, health services cover a range of services (see Table 1). Since this practice model is envisaged to be implemented in primary care services, health services heading practically cover primary care services.

Supplementary material provide the detailed results of this exercise in Table S1, including explanations for each area and examples to illustrate in what ways these are relevant for real-life settings.

Not all of the 24 areas and numerous criteria and issues have the same importance when a practice model is integrated into a health system. Therefore, we organized the areas in two levels: priority and secondary (see the second column in Table 2). If assessment of a priority area doesn't yield a favorable profile in a given country, it will be difficult to integrate the practice model as envisaged, even if it fits in the other areas perfectly. For example, if 'the general environment and culture of the health system' is not favorable for innovations, it would be very difficult to integrated the practice model.

Three of the 'priority' areas are further marked as 'crucial', being a 'must'. These should be the first areas to be investigated in a given country. If they don't yield favorable results, 
investigation of other areas would not be necessary. For example, due to the economic crisis, some countries in Europe are facing drastic budget cuts in public sector (Area 13 in Table 2 and Table S1). In those countries where austerity policies are implemented, it is practically impossible to make a new investment such as the practice model.

Another crucial area we identified is ' 15 . Strength and place of primary care services in the health system and its relationship with other levels of care'. The beneficial effects of the practice model would be most prominent in systems where the primary care physician (GP) has the function to manage the health and risks of individuals registered to his/her practice. If the primary care services are strong and the GPs have a 'gate keeper' position regulating access to specialized care, implementation of the practice model would yield the best results. If it doesn't have the gate-keeper function, it will be very difficult for primary care settings to assume the function of managing the health and risks of individuals and, therefore, application of the practice model wouldn't yield expected benefits. Therefore, we identified that a strong primary care service structure is one of the prerequisites for implementation of this model.

\section{Possible uses and potential benefits of this systematic approach}

The presented systematic approach proposed the use of health system functions as a framework to identify areas to be assessed for integration of an innovative model into a country. It was developed using a preventive personalized health care model as a casestudy. Nevertheless it can be applied to other 'personalized' practices, such as diagnostics, prognostic tools and pharmaceuticals, to be integrated into different health systems.

A comprehensive set of functions of health systems ([16] and Chapter 2) provided the overarching framework to our approach. A previous initiative used another health system framework to analyze integration of health programs in different countries [21, 22]. Although the nature of interventions they took, as well as their purpose of analysis were different, and therefore, not applicable to our case, it indicates that using health systems frameworks to assess integration issues is a credible approach.

When the presented systematic approach is applied to other 'personalized' practices, the functions and, possibly, the sub-functions that should be investigated (Table 1) will stay the same for all cases. However, as it is applied to new cases, each case will identify different set of areas, as well as the specific issues under them. The purpose (e.g. retrospective or prospective analysis), the nature of the practice (intervention), the level of progress towards its integration, and the country settings will also influence the decision on the relevance of different areas.

It might be disappointing for some to see that there is no fixed list of areas related to integration of all 'personalized' practices, which would apply to all countries. An important 
point is to realize that 'one size doesn't fit all' here, either. First of all, 'personalized' practices are not a homogeneous group. When we analyzed the content of 88 'personalized' practices, which were identified by a systematic search in the literature, we found that there are different practices (commodities in health care market or implementation models on how health services are provided), with various purposes (prevention, early detection, diagnosis, treatment, prediction of prognosis, etc.) and serving different groups (individuals/patients, health professionals, health care organizations, etc.) (see Chapter 4). M oreover, countries have diverse health systems. It is not a realistic attempt to develop a single solution that applies to all these diverse cases, purposes and settings in a uniform way. Therefore, instead of a prescriptive approach with a fixed list of issues, we are proposing a systematic approach, where areas and issues related to integration can be identified in a dynamic way, individually for each case.

If this systematic approach is picked up by others and applied on different cases, we encourage them to publish it for two purposes. First of all, exercises carried out on different cases will possibly inspire new ones while identifying areas and issues under them. Secondly, when these different exercises are analyzed in the future, patterns might emerge and enable us to see which areas are important for which cluster of 'personalized' practices. This may lead to developing a number of issues and areas that are highly relevant for certain groups of practices, which can serve as a 'starter set' for the ones to come.

An advantage of approaching the integration issues in a systematic way is that it helps to make sure that no relevant area is missed. It can also help to avoid over-representation of certain perspectives during the efforts to identify the issues and provide a more realistic, and thus possibly more useful, picture of the integration of a practice into health systems.

\section{Concluding remarks}

\section{Enlarge the focus to cover 'health', not only 'disease'}

The main focus of the 'personalized medicine/ personalized health care' field has so far been on diagnosing and treating diseases in a more precise way, with success stories mostly in cancer-related areas. Prevention has been a neglected area in this field. However, 'health' is a broader concept than medicine (diagnosis and therapy) alone. The focus should be enlarged to cover health in a more holistic way, including prevention.

It is of note that prevention is not only early detection of the disease [23] or progression towards disease. Diseases develop gradually in the long years before the onset, where no signs of the disease can be detected, not even molecular ones. Traditionally, the interventions targeting this phase are called 'primary prevention' and usually target the population collectively. However, as we are moving towards a more individualized health care, our focus for this phase should be 'personalized health management'. Innovative tools and implementation models can be used to manage the health of individuals before the onset 
of diseases. For people who have developed risk factors, same strategy can be applied as 'personalized risk management'. In the latter phases, for people who have developed the disease, we can target 'personalized disease management' to prevent complications [24]. These present a huge opportunity to tackle the burden of chronic diseases that is increasing every day.

\section{Establish a dialog between personalized health care and health policy fields}

The field of 'personalized medicine/personalized health care' is very strong in identification of scientific and technological agenda that is needed to discover and develop 'personalized' practices. However, its capacity to approach integration issues in a comprehensive and systematic way is limited. As a solution, the field of health systems and policies has much to offer in provision of the necessary theoretical frameworks and guidance in identification and addressing the issues effectively. In this paper we presented such an attempt to look at the integration issues from the perspective of the field of health systems and policies and this yielded very useful results.

To start working on integration, it is important to establish an effective dialog among the fields of 'personalized medicine/ personalized health care' and health systems and policies so that they can understand each other's worlds and provide effective solutions to the problem of integration into health systems. One way to start this dialog is by using the systematic approach presented in this paper. It may provide the common ground for these fields to be able to convene and work together. As this collaboration identifies issues related to integration of 'personalized' practices correctly in their entirety, the 'barriers' in front them can be overcome effectively.

\section{Supplementary Material}

Table S1. Details of the areas identified for assessment of a country's health system for integration of the practice model. Areas and criteria and issues under them that are identified for assessment of a country's health system for integration of the practice model, as well as comments, examples and preliminary recommendations to countries and the practice model.

\section{References}

1. Hood L, Heath JR, Phelps ME, Lin B. Systems Biology and New Technologies Enable Predictive and Preventative M edicine. Science. 2004 October 22, 2004;306(5696):640-3.

2. Green ED, Guyer MS. Charting a course for genomic medicine from base pairs to bedside. Nature. 2011 Feb 10;470(7333):204-13.

3. Pokorska-Bocci A, Stewart A, Sagoo GS, Hall A, Kroese M, Burton H. 'Personalized medicine': what's in a name? Personalized Medicine. 2014 2014/03/01;11(2):197-210. 
4. Cesuroglu T, van Ommen B, Malats N, Sudbrak R, Lehrach $H$, Brand A. Public health perspective: from personalized medicine to personal health. Personalized Medicine. 2012 2012/03/01;9(2):115-9.

5. Simmons LA, Dinan MA, Robinson TJ, Snyderman R. Personalized medicine is more than genomic medicine: confusion over terminology impedes progress towards personalized healthcare. Personalized M edicine. 2011 2012/01/01;9(1):85-91.

6. Cesuroglu T, Karaca S, Erge S. A practice model for personalized healthcare with a public health genomics perspective. Personalized M edicine. 2009 2009/09/01;6(5):567-77.

7. Snyderman R. Personalized medicine 2014: has healthcare been transformed? Personalized M edicine. 2014 2014/06/01;11(4):365-8.

8. Teng K, Eng C, Hess CA, Holt MA, Moran RT, Sharp RR, et al. Building an innovative model for personalized healthcare. Cleveland Clinic Journal of M edicine. 2012 Apr;79 Suppl 1:S1-9.

9. Overby $\mathrm{CL}$, Tarczy-Hornoch P. Personalized medicine: challenges and opportunities for translational bioinformatics. Personalized Medicine. 2013 Jul 1;10(5):453-62.

10. Jakka $S$, Rossbach $M$. An economic perspective on personalized medicine. Hugo J. 2013;7(1).

11. European Alliance for Personalized Medicine. Innovation and Patient Access to Personalised Medicine - Report from Irish Presidency Conference M arch 20th/21st 2013. Brussels: EAPM; 2013 25.06.2015]. Available from: http://euapm.eu/wp-content/uploads/2012/07/EAPM REPORT-on-Innovation-and-Patient-Access-to-Personalised-M edicine.pdf.

12. Staff NP, Runge BK, Windebank AJ. Breaking Down Translation Barriers: Investigator's Perspective. Sci Transl M ed. 2014 September 3, 2014;6(252):252cm7.

13. Personalized Medicine Coalition. The case for personalized medicine (4th Edition). Washington D.C. (US): PMC; 2014 25.06.2015]. Available from: http:// www.personalizedmedicinecoalition.org/Resources/The_Case_for_Personalized_M edicine.

14. Horgan D, Jansen M, Leyens L, Lal JA, Sudbrak R, Hackenitz E, et al. An index of barriers for the implementation of personalised medicine and pharmacogenomics in Europe. Public Health Genomics. 2014;17(5-6):287-98.

15. World Health Report 2000: Health Systems: Improving Performance. Geneva: World Health Organization; 2000. 206 p.

16. M urray C , Frenk J. A framework for assessing the performance of health systems. B World Health Organ. 2000;78(6):717-31.

17. Roberts MJ, Hsiao W, Berman P, Reich MR. Getting Health Reform Right: A Guide to Improving Performance and Equity. Oxford: Oxford University Press; 2003.

18. Everybody's business: Strengthening health systems to improve health outcomes: WHO's framew ork for action. Geneva: World Health Organization; 2007. 44 p.

19. Atun R, Menabde N. Health systems and systems thinking. In: Coker R, Atun R, McKee M, editors. Health Systems and the Challenge of Communicable Disease: Experiences from Europe and Latin America. European Observatory on Health Systems and Policies Series. Berkshire, England: Open University Press; 2008. p. 121-40.

20. Couzin-Frankel J. U.K. Unveils Plan to Sequence Whole Genomes of 100,000 Patients [News]. 2012. 10 December 2012: [Available from:

http://news.sciencemag.org/scienceinsider/2012/12/uk-unveils-plan-to-sequence-whol.html]. 
21. Atun $\mathrm{R}$, de Jongh $\mathrm{T}$, Secci $\mathrm{F}$, Ohiri $\mathrm{K}$, Adeyi $\mathrm{O}$. Integration of targeted health interventions into health systems: a conceptual framework for analysis. Health policy and planning. 2010 $M$ ar;25(2):104-11.

22. Atun $\mathrm{R}$, de Jongh $\mathrm{T}$, Secci $\mathrm{F}$, Ohiri $\mathrm{K}$, Adeyi $\mathrm{O}$. A systematic review of the evidence on integration of targeted health interventions into health systems. Health policy and planning. 2010 Jan;25(1):1-14.

23. Ilbawi AM, Anderson BO. Cancer in global health: How do prevention and early detection strategies relate? Sci Transl Med. 2015 2015-03-11;7(278):278cm1.

24. The use of health management, risk management, disease management and case management concepts as an alternative to primary, secondary and tertiary prevention classification was developed by Serdar Savas for Diyabet 2020: Vizyon ve Hedefler - Türkiye [Diabetes 2020: Vision and Targets - Turkey]. Istanbul: Turkish Diabetes Foundation, under the auspices of the Turkish $M$ inistry of Health, in collaboration with World Health Organization Regional Office for Europe and International Diabetes Foundation Europe; 2010. 


\section{Supplementary Material}

\section{Background}

A personalized health care model that aims to prevent common chronic diseases was developed by GENAR Institute for Public Health and Genomics Research in Ankara, Turkey [1]. It was identified as a best practice model by the Public Health Genomics European Network in 2008 and piloted in Turkey. It was seen that the main implementation area for such a preventive health care intervention would be wide spread approaches where it is provided within the primary care services in Europe.

However, there has been no example of such a preventive personalized health care model that has been provided to large populations. There was a need for a systematic approach to answer these questions: What are the issues we need to look at when investigating how such a practice model can be integrated to primary care services within health systems? How can we identify them?

In our efforts to answer this question in a systematic way, we used the 'health system framework' that was published earlier [2] as the underpinning of our approach. An adapted version of this framework was presented in Chapter 2. The framework included functions of a health system, which provided the overarching framework to our systematic approach. By using this framework, and our preventive personalized health care model, we carried out an exercise to identify which areas to assess when investigating (if) and how this practice model could be integrated into health systems in Europe. While doing this exercise examples from countries were needed to see what kind of issues can be covered under each area. These were mostly derived from the Netherlands and the UK, since we had access to information on these countries. We used prominent examples from other European contexts as necessary.

\section{Content}

Table S1 presents the results of this exercise. Here, we identified 24 areas that need to be assessed to see how a preventive personalized health care model called Gentest can be integrated into primary care services of health systems in Europe. They are organized under the functions (and sub-functions, as necessary) of health systems, since this had provided the overarching framework to this analysis.

In the table, for each area, first the content and/or the relevance of the area to the topic and/or the case of the practice model is briefly stated.

"Criteria and issues to be considered" list the criteria and issues identified so far by investigation of the practice model, general health system frameworks, relevant literature on related areas, as well as the country examples. This is not 'the' exhaustive list of criteria and issues, but ' $a$ ' list that has been derived so far. As the analysis expands to other coun- 
tries, or goes deeper for given a country/ countries, the list will be likely to be enriched with new items.

"Comments and examples" indicate a general view to the analysis and/or give examples from European settings, in particular from the United Kingdom (UK) and the Netherlands. These also exemplify the relevance of the criteria and issues that have been identified.

Preliminary recommendations are made based on the comments and examples taken and presented mostly in two levels. One is to improve the countries' readiness to integration of such a model, which mostly involve the health system in the macro-level. The second one is the preliminary recommendations made for planning of integration of the practice model. The depth of the recommendations varies, as does the comments and examples.

This systematic approach proposed that the health system functions can be used as a framework to identify areas that need to be assessed for integration of a practice model into a country. Thus, overall approach can be used for a given personalized practice, for its integration to a given country or set of countries. The specific areas will change from case to case and the issues and criteria identified will also change and may grow as it is applied to different country cases. The added value of this paper is not the exhaustive list, but the systematic approach used to identify the broad picture of integration issues under health domain. 
Table S1: Details of the areas identified for assessment of a country's health system for integration of the practice model: Areas and criteria and issues under them that are identified for assessment of a country's health system for integration of the practice model, as well as comments, examples and preliminary recommendations to countries and the practice model.

\section{A. STEWARDSHIP}

\section{Priority setting}

\section{Health policies, strategies and programs}

Health policies as a whole and in its components in forms of strategies must be considered when assessing a country's health system.

\begin{tabular}{|c|c|}
\hline $\begin{array}{l}\text { Criteria and } \\
\text { issues to be } \\
\text { considered }\end{array}$ & $\begin{array}{l}\text { - Existence of coherent written health policy or strategy } \\
\text { - The presence and importance of combating noncommunicable/ chronic } \\
\text { complex diseases in the health policy and strategies } \\
\text { - Orientation of the health system and policies towards prevention in individual } \\
\text { and population level } \\
\text { - Within the health policy, strategies and programs, presence and role of } \\
\text { o Personalized medicine and health care as a whole or with its compo- } \\
\text { nents } \\
\text { o Technologies that are used in the practice model, such as genomics and } \\
\text { information and communication technologies }\end{array}$ \\
\hline $\begin{array}{l}\text { Comments } \\
\text { and examples }\end{array}$ & $\begin{array}{l}\text { - The European Union (EU) doesn't have a unified single health policy that } \\
\text { covers all EU countries in all aspects. Each member state is individually re- } \\
\text { sponsible for organization and delivery of health services and medical care } \\
\text { (Lisbon Treaty, } 2007 \text { [3]). } \\
\text { - On the other hand, the EU Health Strategy document (2007) [4] highlights } \\
\text { some strategies for 'cooperative action at Community level'. "Supporting Dy- } \\
\text { namic Health Systems and New Technologies" is one of the objectives of the } \\
\text { Commission within this strategy. This highlights new technologies such as e- } \\
\text { health, genomics and biotechnologies, and addresses evaluation including } \\
\text { cost effectiveness and equity, health professionals' training and capacity im- } \\
\text { plications and possible ethical concerns. } \\
\text { The European Strategy for the Prevention and Control of Noncommunicable } \\
\text { Diseases by World Health Organization Europe (2006) [5] has indicated that } \\
\text { "The personal risk of developing disease can be dependent on the interaction } \\
\text { between the individual, his or her personal susceptibility and the wider envi- } \\
\text { ronment". The report highlights that increasing number of tests will be de- } \\
\text { veloped for genetic predisposition to common diseases and introduction of } \\
\text { these tests into health care “...is likely to have significant consequences for } \\
\text { the organization, staffing and delivery of health services, as well as to raise is- } \\
\text { sues of ethics and equity of access." }\end{array}$ \\
\hline
\end{tabular}




\begin{tabular}{|l|l|}
\hline & $\begin{array}{l}\text { Country level applications are heterogeneous. Target countries' health policy } \\
\text { should be considered individually, using various documents that indicate the } \\
\text { policy directions in the country, such as policy documents, strategies and } \\
\text { programs. }\end{array}$ \\
- $\begin{array}{l}\text { For example, in England, in the public health policy paper (Healthy Lives, } \\
\text { Healthy People: our strategy for public health in England, 2010) [6] there is no } \\
\text { reference to personalized health care, other personalized approaches or pub- } \\
\text { lic health genomics. In this document, references to modern biology are also } \\
\text { very rare [7]. } \\
\text { - Another recent example is from the Netherlands. Dutch Government has } \\
\text { identified more personal, customized services and better prevention among } \\
\text { the six tasks of the planned transformation of the social domain [8]. Other } \\
\text { tasks include, among others, 'less escalation to primary and secondary care } \\
\text { and support' and 'more integration'. These indicate the increasing emphasis } \\
\text { on prevention and personalization in the health related strategies in the } \\
\text { Netherlands. }\end{array}$ \\
\hline $\begin{array}{l}\text { Preliminary } \\
\text { recommen- } \\
\text { dations }\end{array}$ & $\begin{array}{l}\text { To improve countries' readiness: } \\
\text { In order to address the major transformation in health care that we may face } \\
\text { inth personalized health care applications, countries need to take into ac- } \\
\text { count this issue proactively in their overall health strategies [9]. } \\
\text { It is important that the country for integration is a 'breeding ground' for } \\
\text { development and implementation of personalized health care models. This } \\
\text { can be achieved by including personalized health care and its critical success } \\
\text { factors within health policies. }\end{array}$ \\
\hline
\end{tabular}

\section{Strategies on relevant applications and technologies}

Some countries may have separate strategies, programs or initiatives for relevant applications (such as practice models in personalized health care, e-health, etc.) and/or technology fields (human genomics, information and communication technologies in health care, biotechnology for health, etc.).

\begin{tabular}{|l|ll|}
\hline $\begin{array}{l}\text { Criteria and } \\
\text { issues to be } \\
\text { considered }\end{array}$ & $\begin{array}{l}\text { Presence and content of a strategy or program on one or more of the applica- } \\
\text { tion areas and/or technology fields (as described above) }\end{array}$ \\
\hline $\begin{array}{l}\text { Comments } \\
\text { and examples }\end{array}$ & $\begin{array}{l}\text { - } \begin{array}{l}\text { Stakeholder involvement in development of the strategy } \\
\text { Endorsement and/or implementation of the strategy in actual life }\end{array} \\
\text { itance - Genomic technology in healthcare" in the UK [10] is an example to } \\
\text { such policies. }\end{array}$ \\
& $\begin{array}{l}\text { The 'Health initiative' of the Luxembourg government which aims to trans- } \\
\text { form Luxembourg into a center of excellence in the area of personalized med- } \\
\text { icine [11] and in its continum, the country's vision to become "the first na- } \\
\text { tion in the world in which every person has an opportunity to avoid prevent- } \\
\text { able diseases, to access effective diagnostic and therapeutic care for curable }\end{array}$ \\
\hline
\end{tabular}




\begin{tabular}{|l|c|}
\hline & $\begin{array}{c}\text { diseases, and to adapt and self-manage when faced with unavoidable sources } \\
\text { of suffering." [12] are examples to comprehensive strategies in this area. }\end{array}$ \\
\hline $\begin{array}{l}\text { Preliminary } \\
\text { recommen- } \\
\text { dations }\end{array}$ & $\begin{array}{l}\text { To improve countries' readiness: } \\
\text { - If and when possible, creating strategic and cross-cutting programs with the } \\
\text { involvement of relevant stakeholders can enable turning research and devel- } \\
\text { opment into implementation in the fragmented environment of science, } \\
\text { technology and health care. }\end{array}$ \\
\hline
\end{tabular}

\section{Legislation and regulations}

\section{Regulation of non-diagnostic services which aim prevention}

The practice model is not a diagnostic tool since it aims to prevent diseases. Therefore, it may not fall into a clear area of regulation. For this purpose, the regulations should be investigated from different angles. One of them is to investigate if there are any regulations that cover services that aim prevention but not diagnosis.

\begin{tabular}{|c|c|}
\hline $\begin{array}{l}\text { Criteria and } \\
\text { issues to be } \\
\text { considered }\end{array}$ & $\begin{array}{l}\text { - Presence of specific regulations that regulate services or tests that aim pre- } \\
\text { diction and prevention but not diagnosis } \\
\text { - } \quad \text { If there are any restrictions in other legislative documents } \\
\text { - } \quad \text { Any related guidelines }\end{array}$ \\
\hline $\begin{array}{l}\text { Comments } \\
\text { and examples }\end{array}$ & $\begin{array}{l}\text { - In the Netherlands, "Wet op het bevolkingsonderzoek", "Act on population } \\
\text { screening" [13] regulate use of tests for screening of diseases, not diagnosis. } \\
\text { The main aim of this regulation is to prevent burden that might be brought by } \\
\text { unnecessary screening programs. } \\
\text { - The primary aim of Gentest is identification of risk factors and risks, not early } \\
\text { diagnosis of diseases. However, various components of Gentest may lead to } \\
\text { early diagnosis of diseases. For example, blood glucose levels higher than } 121 \\
\text { mg/dL will lead to, if replicated, diagnosis of diseases. Therefore, this regula- } \\
\text { tion may have various implications of how the practice model is positioned } \\
\text { and organized within health services in the Netherlands. } \\
\text { - The Royal Dutch Medical Association (KNM G) has already issued a multidisci- } \\
\text { plinary guideline on 'preventive medical examinations' in 2013, to guide doc- } \\
\text { tors on the principles of 'check-up' programs they will provide [14]. } \\
\text { Fueled by the introduction of 'total body scans' in the Netherlands, Health } \\
\text { Council of the Netherlands published an advisory report on population } \\
\text { screening, which included the criteria that must be met by the 'health } \\
\text { checks', in } 2015 \text { [15]. } \\
\text { All these have important implications on how Gentest can be offered in the } \\
\text { Netherlands. }\end{array}$ \\
\hline $\begin{array}{l}\text { Preliminary } \\
\text { recommen- } \\
\text { dations }\end{array}$ & $\begin{array}{l}\text { For integration of the practice model: } \\
\text { - With the help of public authorities and health lawyers, it should be made } \\
\text { clear if and what parts of Gentest falls into the scope of the "Act on popula- } \\
\text { tion screening" in the Netherlands. In addition, the criteria brought by the }\end{array}$ \\
\hline
\end{tabular}




\begin{tabular}{|l|l|}
\hline Health Council on heath checks must be investigated. Strategies should be \\
developed to make the implementation compliant with the law and the crite- \\
ria of the Health Council. If there are fundamental principles that explain why \\
some parts are not compliant, it should be made clear and well-articulated. \\
- Implementation of Gentest should be carried out in compliance with guide- \\
line on 'preventive medical examinations' in the Netherlands. \\
To improve countries' readiness: \\
Our practice model, Gentest, is not a conventional approach among health \\
interventions. When regulations are made, an umbrella regulation might \\
have been developed to prevent harm from specific practices; which may \\
create a barrier towards implementation of Gentest. This illustrates that \\
regulations should be made to protect the individuals, while giving the room \\
for innovations that may benefit them to be implemented.
\end{tabular}

\section{Regulation of genetic testing, including predictive testing for imple- mentation in primary care services}

Genetics is one of the inputs of the practice model. In many countries, genetic testing has a specific legislation, which mainly targets genetic diagnosis. However, the practice model in question doesn't involve diagnosis. Nevertheless, relevant regulations should be investigated to see if there are any restrictions for implementing a model which contains genetic factors as one of its components.

\begin{tabular}{|l|l|l|}
\hline $\begin{array}{l}\text { Criteria \& } \\
\text { issues to be } \\
\text { considered }\end{array}$ & $\begin{array}{l}\text { Any restriction on use of genetic data for a practice model implemented in } \\
\text { primary care }\end{array}$ \\
\hline $\begin{array}{l}\text { Comments } \\
\text { and examples }\end{array}$ & $\begin{array}{l}\text { The genetic testing for prevention of common complex diseases is mostly } \\
\text { considered to be predictive genetic testing, and due to direct-to-consumer } \\
\text { (DTC) tests in Europe and mainly in the United States of America (USA) avail- } \\
\text { able via internet, there is an increasing sensitivity in Europe on genetic tests } \\
\text { outside medical genetics. There are several reports on views on regulation of } \\
\text { DTC tests (such as European Society of Human Genetics' statement in 2010 } \\
\text { [16] and European Academies Science Advisory Council and Federation of Eu- } \\
\text { ropean Academies of Medicine's report in 2012 [17]). It should be noted that } \\
\text { the practice model in question is not a direct-to-consumer test since it will be } \\
\text { implemented by a medical doctor, i.e. a general practitioner. } \\
\text { Due to the concerns over DTC tests, it seems that more stringent regulations } \\
\text { have been entering into force in Europe in the past years. For example, in } \\
\text { Germany, diagnostic and predictive genetic tests can be conducted only by } \\
\text { medical doctors who are certified specialists in human genetics as set forth by } \\
\text { the law [18, 19]. Several aspects needs to be explored to identify if a practice } \\
\text { model which aims to prevent complex diseases and utilizes genetic factors in } \\
\text { combination with other markers in primary care services would fit in that } \\
\text { regulatory framework. An important discussion would be how the genetic in- } \\
\text { formation obtained in the context of practice model would be classified ac- }\end{array}$ \\
\hline
\end{tabular}




\begin{tabular}{|c|c|}
\hline & $\begin{array}{l}\text { cording to the laws. } \\
\text { - Target countries' legislation on genetic testing should be considered individu- } \\
\text { ally. }\end{array}$ \\
\hline $\begin{array}{l}\text { Preliminary } \\
\text { recommen- } \\
\text { dations }\end{array}$ & $\begin{array}{l}\text { To improve countries' readiness: } \\
\text { - } \\
\text { One of the foundation principles of primary care services is to provide a } \\
\text { Alma-Ata in 1978. It is the first level of contact of individuals, the family and } \\
\text { community with the national health system bringing health care as close as } \\
\text { possible to where people live and work, and constitutes the first element of a } \\
\text { continuing health care [20, 21]. Prevention is one of the core functions of } \\
\text { primary care services. Therefore, prevention of complex diseases would defi- } \\
\text { nitely fall under the scope of primary care services. In the practice model, ge- } \\
\text { netic factors function as one of the inputs, but not the main purpose. Thus, as } \\
\text { in many practices of primary care services, several different types of inputs } \\
\text { are considered. } \\
\text { If the regulatory framew ork that restricts ordering and interpretation of } \\
\text { genetic tests would be a barrier for implementation of this practice model, } \\
\text { which includes genetic factors among the several parameters, in primary } \\
\text { care, this would be contradictory to the underlying philosophy of primary } \\
\text { care services. } \\
\text { In addition, the next question would be: Why is the genetic part of the prac- } \\
\text { tice model special? As a doctor, why can the primary care physician process } \\
\text { all the relevant information of the individual to prevent the complex diseases, } \\
\text { but not the genetic factors? This also would match with the criticisms on } \\
\text { German law for practicing 'genetic exceptionalism' [22, 23]. } \\
\text { If all the aspects of the practice model would be interpreted by relevant } \\
\text { specialty fields, such as genetic aspects by medical geneticist, cardiologists in } \\
\text { charge of assessment and recommendations on cardiovascular diseases, dia- } \\
\text { betologist in charge of diabetes, etc., it would not only create fragmentation, } \\
\text { but also lead to huge inefficiency. } \\
\text { of primary care services in the overall health system. }\end{array}$ \\
\hline
\end{tabular}

\section{Regulations on ethical issues in terms of implementation}

The practice model involves risk assessments for common chronic diseases. Currently, the genetic information is not included in the risk assessment algorithms within the practice model. It is used only for prioritization and tailoring of the lifestyle factors. However, as the scientific developments progresses, it is expected that various types of genome-based information can be incorporated to risk assessment algorithms.

With or without use of genomic markers, assessment of risk of complex diseases may require ethical issues to be considered. In addition, the use of genetic information in the model requires compliance to ethical principles on genetic testing for human health. As the main principle, the 


\begin{tabular}{|c|c|}
\hline $\begin{array}{l}\text { Criteria and } \\
\text { issues to be } \\
\text { considered }\end{array}$ & $\begin{array}{l}\text { - } \quad \text { Any ethical restrictions on this kind of service } \\
\text { - } \quad \text { The need for informed consent } \\
\text { - } \quad \text { Any possible effects of family involvement }\end{array}$ \\
\hline $\begin{array}{l}\text { Comments } \\
\text { and examples }\end{array}$ & $\begin{array}{l}\text { - The informed consent must be obtained as set forth in the national regula- } \\
\text { tions of each country. For example, if the consent form must be signed in } \\
\text { presence of a doctor and/or co-signed by a doctor, this needs to be taken into } \\
\text { account. } \\
\text { - Primary care services not only provide a holistic approach to the individual, } \\
\text { but also the family as a whole. The family members not only share their DNA, } \\
\text { but also most lifestyle factors and living conditions. In addition, in terms of } \\
\text { implementation of the practice model, family involvement can play an im- } \\
\text { portant role to increase the success rate of implementing the lifestyle rec- } \\
\text { ommendations. On the other hand, the family involvement may also lead to } \\
\text { pressure on the individuals. These ethical issues must be considered when } \\
\text { outlining the service delivery models. }\end{array}$ \\
\hline $\begin{array}{l}\text { Preliminary } \\
\text { recommen- } \\
\text { dations }\end{array}$ & $\begin{array}{l}\text { For integration of the practice model: } \\
\text { - } \quad \text { since implementation of the practice model will involve large numbers of } \\
\text { people, other types of informed consent (waiver of documentation of in- } \\
\text { formed consent and implied consent) can be considered for practices that are } \\
\text { not related to diagnosis of (monogenic) diseases. } \\
\text { To improve countries' readiness: } \\
\text { - Ethical frameworks on genetic testing should consider use of genetic infor- } \\
\text { mation not only for diagnostic tests, but also models which utilize genetic in- } \\
\text { formation as one of its several components. }\end{array}$ \\
\hline
\end{tabular}

\section{Regulations on liability of the practice model}

As a report which involves health related advice, the liability of the assessments and recommendations made in the report must be ensured.

\begin{tabular}{|l|ll|}
\hline $\begin{array}{l}\text { Criteria and } \\
\text { issues to be } \\
\text { considered }\end{array}$ & $\begin{array}{l}\text { - } \begin{array}{l}\text { Any legislative framework available on liability of } \\
\text { Comments } \\
\text { and examples }\end{array} \\
\text { cow liability issues are handled in examples of other applications, such as } \\
\text { cardiovascular (CV) risk assessment programs and tele-health applications }\end{array}$ \\
& $\begin{array}{l}\text { If there is a legislative framework available in the country on the liability of } \\
\text { such practice models, assessment tools or health recommendations, that as- } \\
\text { sist medical doctors, nurses, dieticians and other health professionals, these } \\
\text { must be considered. }\end{array}$ \\
& $\begin{array}{l}\text { However, it is very likely that there is no ready clear legal framew ork for this } \\
\text { issue in many countries. In that case, the legal framework will need to be es- } \\
\text { tablished by the health authorities and decision makers. } \\
\text { If there is no legislative framework, examples of applications of CV risk as- } \\
\text { sessment and tele-health can also be investigated to see how liability issues }\end{array}$ \\
\hline
\end{tabular}




\begin{tabular}{|l|l|}
\hline & are handled. \\
\hline $\begin{array}{l}\text { Preliminary } \\
\text { recommen- }\end{array}$ & $\begin{array}{l}\text { For integration of the practice model: } \\
\text { Itions }\end{array}$ \\
& $\begin{array}{l}\text { It is important to make early contact with health authorities to start discus- } \\
\text { sion on such matters, including liability. } \\
\text { An option can be assigning the liability to the Chief M edical Officer and/or the } \\
\text { M edical Advisory Board that needs to be established within the enterprise } \\
\text { that will implement the practice model in Europe. } \\
\text { In the consent form, it should be made sure that it writes that on one hand, } \\
\text { applying the lifestyle advice doesn't eliminate the risk of diseases and on the } \\
\text { other hand, the risk assessments doesn't indicate any certainty if the person } \\
\text { will get the disease or not. }\end{array}$ \\
\hline
\end{tabular}

\section{Regulations on data protection, privacy and confidentiality}

As in all health related applications, the issues on data protection, privacy and confidentiality must be considered and safeguarded.

\begin{tabular}{|c|c|}
\hline $\begin{array}{l}\text { Criteria and } \\
\text { issues to be } \\
\text { considered }\end{array}$ & $\begin{array}{l}\text { - The general framew ork of data protection, privacy and confidentiality in } \\
\text { Europe and of the target country. Some specific aspects that need attention, } \\
\text { among others: } \\
\text { o Informed consent } \\
\text { o Data collection and use; including purpose, data storage duration } \\
\text { o Rights of the data subjects } \\
\text { - Measures required for security and confidentiality of data processing } \\
\text { - Need for data protection impact assessment and assignment of a data pro- } \\
\text { tection officer } \\
\text { Other national issues that are related to data protection, privacy and confi- } \\
\text { dentiality in health services }\end{array}$ \\
\hline $\begin{array}{l}\text { Comments } \\
\text { and examples }\end{array}$ & $\begin{array}{l}\text { - EU Directive on Data Protection [24] provides an important framework for } \\
\text { this area. On the other hand, the regulations and practices of each country } \\
\text { differ in the current situation, making it necessary to investigate each country } \\
\text { individually. } \\
\text { - The proposed regulation on Data Protection [25] may be expected to lead to } \\
\text { harmonization of data protection issues in Europe and a level playing field in } \\
\text { all member states but the issues that will come up in the actual practice after } \\
\text { the adoption of the regulation is difficult to be foreseen for each country. } \\
\text { Therefore, each country must be investigated individually. }\end{array}$ \\
\hline $\begin{array}{l}\text { Preliminary } \\
\text { recommen- } \\
\text { dations }\end{array}$ & $\begin{array}{l}\text { For integration of the practice model: } \\
\text { - The principles and obligations in relation to data protection legislation should } \\
\text { be identified for each country for provision of the practice model in primary } \\
\text { care services. } \\
\text { - The proposed regulation on Data Protection has been heavily criticized by }\end{array}$ \\
\hline
\end{tabular}




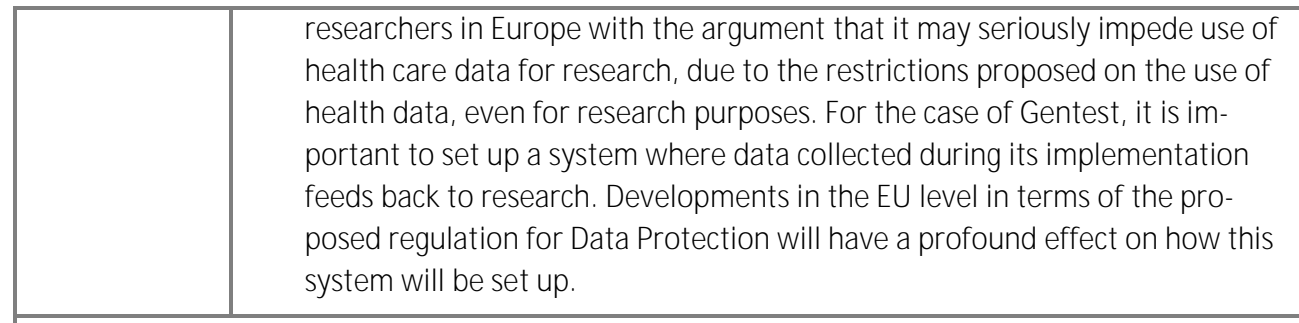

\section{Regulations on cross-border health care}

Directive on cross-border healthcare was adopted by the Council of the European Union in 2011. Member states must transpose the directive's provision into national health legislation. The directive meets the needs on patients' rights in cross-border healthcare while preserving member states' rights to organize their own health systems [26].

\begin{tabular}{|c|c|}
\hline $\begin{array}{l}\text { Criteria and } \\
\text { issues to be } \\
\text { considered }\end{array}$ & $\begin{array}{l}\text { - Possible consequences of the cross-border regulation for implementation of } \\
\text { this practice model in primary care services }\end{array}$ \\
\hline $\begin{array}{l}\text { Comments } \\
\text { and examples }\end{array}$ & $\begin{array}{l}\text { - The implementation of the practice model will be embedded in primary care } \\
\text { services. The practice model is envisaged to be an integral part of the health } \\
\text { record of the individual in primary care services. } \\
\text { - As a definition, primary care services is the first level of contact of individuals, } \\
\text { the family and community with the national health system bringing health } \\
\text { care as close as possible to where people live and work, and constitutes the } \\
\text { first element of a continuing health care process [20]. Unlike some other are- } \\
\text { as of health care, primary care services is not a one-off type of service to be } \\
\text { used. In many countries, a person must enroll to a primary care service pro- } \\
\text { vider, usually a general practitioner or family physician (GP). In line with the } \\
\text { definition of primary care services, geographical proximity and logistics plays } \\
\text { a very important role in choosing a primary care provider [27]. Although the- } \\
\text { oretically possible, it would be very questionable for individuals to enroll to a } \\
\text { GP in a cross border setting. } \\
\text { Primary care services are organized in the level of countries and, to a certain } \\
\text { extent, regions within countries (federal states or governments). It would be } \\
\text { fairly unlikely for the resident of one country to enroll to a GP in another } \\
\text { country because individuals want to be treated as close to home as possible } \\
\text { in a system they feel familiar with [28]. }\end{array}$ \\
\hline $\begin{array}{l}\text { Preliminary } \\
\text { recommen- } \\
\text { dations }\end{array}$ & $\begin{array}{l}\text { For integration of the practice model: } \\
\text { - As different cases arise in terms of implementation of this directive in Euro- } \\
\text { pean countries, their implications on the case of Gentest should be consid- } \\
\text { ered. }\end{array}$ \\
\hline
\end{tabular}




\section{Steering}

\section{General environment and culture of the health system for innovation}

The main added value of the practice model in question is not the technologies utilized (genetic technologies, information and communication technologies, tools and techniques on measurement of lifestyle factors, etc.), but how the information is analyzed and synthesized in an integrative and holistic way for the purpose of prevention of complex diseases in individual level. Thus, it should be regarded as an 'innovation', rather than a 'technology' per se.

The practice model conflicts with the current mindset in the health sector in some aspects, including the following:

- The classical paradigm stipulates that "Prevention can be done either in population level, targeting primary prevention (population strategy), or individual level, targeting secondary prevention (high-risk strategy)." (this paradigm dates back to Rose, 1985 [29]). However, the practice model brings a new approach: population wide (inclusive) prevention in individual level. This doesn't comply with the 'either-or' paradigm stated in the first sentence.

- Moreover, in the classical paradigm, primary prevention interventions almost always target the population level. The practice model also challenges this classical paradigm by providing individual level services for primary prevention. Thus, to understand and integrate the mod$\mathrm{el}$, the stakeholders and health authorities need to 'think out of the box'.

These new aspects not only require putting a new application in practice, but also some change in the perspective and mind-set of the stakeholders. In addition, reorganization or redesign of preventive services in primary care will highly likely be required for successful implementation of the model. It also requires change management and, to some extent, social engineering focusing on health sector.

\begin{tabular}{|c|c|}
\hline $\begin{array}{l}\text { Criteria and } \\
\text { issues to be } \\
\text { considered }\end{array}$ & $\begin{array}{l}\text { - The attitude of the health policies and strategies on the need for innovation } \\
\text { - The openness of health sector in general and health authorities to innovation } \\
\text { (recent new applications in health sector might be taken as a case or if there } \\
\text { are } \\
\text { - The actual implementation of innovations in health sector } \\
\text { - Barriers against implementation of innovations }\end{array}$ \\
\hline $\begin{array}{l}\text { Comments } \\
\text { and examples }\end{array}$ & $\begin{array}{l}\text { - In European level, there are programs addressing the need for innovation for } \\
\text { specific contexts, such as European Innovation Partnership on Active and } \\
\text { Healthy Ageing (established in 2011, targeting } 2020 \text { [30]). } \\
\text { - An example to country level programs is the UK's program on 'Innovation, } \\
\text { Health and Wealth, Accelerating Adoption and Diffusion in the National } \\
\text { Health Service (NHS)'. It sets out an integrated set of measures that together } \\
\text { will support the adoption and diffusion of innovation across the NHS and sets } \\
\text { a delivery agenda that will significantly ramp up the pace and scape of change } \\
\text { and innovation [31]. } \\
\text { In terms of willingness to implement new technologies, the UK's plan to make } \\
\text { whole genome sequencing on } 100.000 \text { patients with cancer and rare diseases }\end{array}$ \\
\hline
\end{tabular}




\begin{tabular}{|c|c|}
\hline & $\begin{array}{l}\text { can be taken as an example [32]. The aim is "to make UK world genetic re- } \\
\text { search leader", as announced by the Prime Minister David Cameron [33]. } \\
\text { Among the main aims of the project is to kickstart the development of a UK } \\
\text { genomics industry. These signal a 'pro-genomics' environment for integration } \\
\text { of genome-based technologies into health services. } \\
\text { - There is no available 'scale' that determines willingness to accept innovation } \\
\text { in health care sector for each country. The surveys on innovation and health } \\
\text { sector innovation can be used as data sources to some extent. } \\
\text { The countries' response to 'tele-health' applications can be considered as } \\
\text { examples that can be studied because it also requires changing the mind sets } \\
\text { and redesigning services for prevention (tertiary prevention). }\end{array}$ \\
\hline $\begin{array}{l}\text { Preliminary } \\
\text { recommen- } \\
\text { dations }\end{array}$ & $\begin{array}{l}\text { To improve countries' readiness: } \\
\text { - Understanding the need for innovation in health systems is a key for reducing } \\
\text { the costs of health care while increasing the life expectancy and quality of } \\
\text { life. }\end{array}$ \\
\hline
\end{tabular}

\section{Stakeholders, processes and mechanisms involved in implementation of new applications in health care}

Countries' health systems have different approaches to implementation of new technologies in health care with involvement of different stakeholders and processes. There is also variability in stakeholders and processes among different types of applications (prevention, diagnosis, screening, etc.) in different settings (primary care, hospitals, etc.).

As stated under ' 9 . General environment and culture of the health system for innovation', the practice model utilizes several different types of information which interact each other and analyses and synthesizes them in several layers, making it a 'complex intervention'[34]. The complexity of the intervention also leads to involvement of various disciplines and stakeholders, making the process of putting the model into practice also complex. It is important to understand the stakeholders, processes and mechanisms involved in implementation a new application such as the practice model.

It is important to note that there is no such application that has been developed and implemented in a health system in Europe, i.e. a personalized health care model utilizing several types of information on the individual in an integrative way with the purpose of prevention of complex diseases and implemented in primary care services. Therefore, cases which have some common properties can be investigated as examples. These include e-health and tele-health applications, application of services which involve genetic/genomic testing (such as pharmacogenomics), and cardiovascular (CV) risk assessment and management programs.

\begin{tabular}{|c|c|}
\hline $\begin{array}{l}\text { Criteria and } \\
\text { issues to be } \\
\text { considered }\end{array}$ & $\begin{array}{l}\text { - Stakeholder analysis } \\
\text { - If known, positions of stakeholders about various aspects of the model, } \\
\text { including service redesign in primary care services, use of individual level in- } \\
\text { terventions for primary prevention, use of genomic technologies and infor- } \\
\text { mation and communication technologies in health care } \\
\text { - Study of cases that have similarities in some aspects to the practice model (as }\end{array}$ \\
\hline
\end{tabular}




\begin{tabular}{|c|c|}
\hline & $\begin{array}{l}\text { - Stated above) } \\
\text { Study of cases on how a recently new technology with complex interventions } \\
\text { has been implemented or prepared in the health system, preferably in prima- } \\
\text { ry care services }\end{array}$ \\
\hline $\begin{array}{l}\text { Comments } \\
\text { and examples }\end{array}$ & $\begin{array}{l}\text { - Each target country should be considered individually. Focus should be on } \\
\text { examples of preventive applications (not preferably tests) in primary care } \\
\text { services. Data would not be readily available on various aspects of the crite- } \\
\text { ria. Therefore, one or more studies will need to be carried out. } \\
\text { - For example, in the UK, the case of implementation of tele-health in primary } \\
\text { care can be taken as a case example [35-39]. For the Netherlands, CV risk as- } \\
\text { sessment and management programs that have been introduced in GP prem- } \\
\text { ises can be investigated as a case example. }\end{array}$ \\
\hline $\begin{array}{l}\text { Preliminary } \\
\text { recommen- } \\
\text { dations }\end{array}$ & $\begin{array}{l}\text { For integration of the practice model: } \\
\text { - It is important to know the stakeholders, processes and mechanisms in each } \\
\text { country in general and specifically for primary care services and prevention. }\end{array}$ \\
\hline
\end{tabular}

\section{Administration and management}

\section{Mechanisms related to evaluation of new applications and decision making in health care services}

While supporting innovation, it requires a balancing act on the side of the decision makers to protect the citizens, maintain the health care budgets (of all kinds of third party payers), and allow and promote innovation in health systems. Mechanisms related to evaluation of new applications and decision making can indicate how well this balance can be achieved in the given health system.

\begin{tabular}{|c|c|}
\hline $\begin{array}{l}\text { Criteria and } \\
\text { issues to be } \\
\text { considered }\end{array}$ & $\begin{array}{l}\text { - Presence and functions of a body or mechanism for proactive horizon screen- } \\
\text { ing for scientific and technological developments in health care "Early aware- } \\
\text { ness and alert systems" (for examples, see EuroScan [40]) } \\
\text { - The process for authorization of an application for market entry } \\
\text { - The process for an application to be covered by a third party payer (If national } \\
\text { health system type finance, it is national health budget. If social health insur- } \\
\text { ance type finance, it is the budget of the each social health insurance fund } \\
\text { and/or the national reimbursement lists) } \\
\text { - Utilization of Health Technology Assessment (HTA) } \\
\text { - Existing HTA reports on applications which have some common properties, } \\
\text { such as complex interventions that require service redesign in primary care } \\
\text { services, use of individual level interventions for primary prevention, use of } \\
\text { genomic technologies and information and communication technologies in } \\
\text { health care } \\
\text { - The country's general level of 'strictness' in decision making } \\
\text { Recent decisions on implementation of new applications (as much as possible } \\
\text { which have similarities to the practice model, as pointed out in } \\
\text { 10.Stakeholders, processes and mechanisms involved in implementation of }\end{array}$ \\
\hline
\end{tabular}




\begin{tabular}{|c|c|}
\hline & $\begin{array}{l}\text { new applications in health care) and how those decisions were made } \\
\text { - } \quad \text { Evaluative framework implemented in that country } \\
\text { - } \quad \text { Economic evaluation methods utilized } \\
\text { - } \quad \text { Decision making when there is an evidence gap for promising applications } \\
\text { which are evaluated as 'safe' } \\
\text { - Decision makers early involvement in development of new technologies [41] }\end{array}$ \\
\hline $\begin{array}{l}\text { Comments } \\
\text { and examples }\end{array}$ & $\begin{array}{l}\text { - There is an International Information Network on New and Emerging Health } \\
\text { Technologies (EuroScan International Network [40]). } \\
\text { Some countries have horizon screening bodies, such as NIHR HSC (The Na- } \\
\text { tional Institute for Health Research Horizon Scanning Centre [42]), and others } \\
\text { have this function covered by other agencies such as HTA agencies. Functions } \\
\text { can be investigated for each target country. } \\
\text { The process experienced in the UK on guidance development for gene ex- } \\
\text { pression profiling and expanded immunohistochemistry tests to guide the use } \\
\text { of adjuvant chemotherapy in early breast cancer management (M ammaPrint, } \\
\text { Oncotype DX, IHC4 and Mammostrat) can be used for comparison, for exam- } \\
\text { ple. It seems that, for example, the UK may have a more strict approach on } \\
\text { evaluation of (cost-) effectiveness compared to other countries [43]. } \\
\text { Some countries have a specific evaluative framework and a specific perspec- } \\
\text { tive and method in terms of economic evaluation. For example, the UK (NICE } \\
\text { - The National Institute for Health and Care Excellence) has a clear perspec- } \\
\text { tive, which is 'health budget perspective' and doesn't consider any benefits } \\
\text { other than health at the economic analysis and decision making. In relation to } \\
\text { this, the preferred method for economic evaluation is cost-effectiveness } \\
\text { analysis. [44] } \\
\text { On the other hand, Sweden (Swiss HTA) have a prior normative commitment } \\
\text { and consider also 'social preferences' perspective such as non-discrimination, } \\
\text { protection of autonomy, equal access to appropriate health care, effectively } \\
\text { maintaining or restoring health-related quality of life, functioning and capa- } \\
\text { bilities. In relation to this, they support methodological pluralism and decide } \\
\text { the most appropriate evaluation method based on the specific research ques- } \\
\text { tion [45]. (If benefits other than health are to be included in the analysis, } \\
\text { as 'safe' [46]. (Also, amone Khourt is the most likely analysis method to be used) } \\
\text { Khoury et al, 2012 [47]). The presence of this option is very important since } \\
\text { the practice model is highly likely to fall into this category, if such a category } \\
\text { exists. }\end{array}$ \\
\hline $\begin{array}{l}\text { Preliminary } \\
\text { recommen- } \\
\text { dations }\end{array}$ & $\begin{array}{l}\text { For integration of the practice model: } \\
\text { - } \quad \text { Communication with decision makers (decision makers including public }\end{array}$ \\
\hline
\end{tabular}




\begin{tabular}{|l|l|}
\hline & $\begin{array}{l}\text { authorities and third party payers) and their involvement should be initiated } \\
\text { in the early stages of the preparations for evaluation of the practice model } \\
\text { for specific countries. }\end{array}$
\end{tabular}

\section{B. FINANCING ${ }^{1}$}

\section{Health financing system of the country}

The general picture about the financial system and expenditures of the country needs to be known.

\begin{tabular}{|c|c|}
\hline $\begin{array}{l}\text { Criteria and } \\
\text { issues to be } \\
\text { considered }\end{array}$ & $\begin{array}{l}\text { - General resource generation and financing system (national health system, } \\
\text { - Tocial health insurance, others) } \\
\text { es financing system specific to primary care services, particularly GP premis- } \\
\text { - The financing system from the perspective of prevention of complex diseases, } \\
\text { in particular, the payer and the benefiter of investments in health care ser- } \\
\text { vices }\end{array}$ \\
\hline $\begin{array}{l}\text { Comments } \\
\text { and examples }\end{array}$ & $\begin{array}{l}\text { - In terms of health systems and policies, Europe has a diverse profile. There- } \\
\text { fore, each target country must be investigated individually. In a very broad } \\
\text { sense, there are national health service (NHS) based systems which are fund- } \\
\text { ed from general taxation (such as the UK), and social health insurance (SHI) } \\
\text { based systems which are mainly funded from wage related contribution (such } \\
\text { as Germany). It is of note that, in general, there is a trend of convergence be- } \\
\text { tween NHS and SHI [48, 49]. } \\
\text { - The type of the financing of the health system can have an impact on how } \\
\text { new applications are evaluated and the decision making processes (see also } \\
\text { 11. M echanisms related to evaluation of new applications and decision mak- } \\
\text { ing in health care services). } \\
\text { From technology perspective, in principle, there should be two thresholds for } \\
\text { a technology in a health system. The first threshold is for entering the health } \\
\text { care market, indicating that a technology is safe enough to enter the market. } \\
\text { Second one is the threshold for financial coverage (reimbursement) indicating } \\
\text { that the technology is cost-effective enough for the costs to be covered by a } \\
\text { third party payer. } \\
\text { In the UK, NHS, for example, these two thresholds practically merged because } \\
\text { there is a national single payer, who also decides on cost effectiveness. If sin- } \\
\text { gle payer doesn't cover this technology due to lack of cost effectiveness, } \\
\text { practically, the technology doesn't have a place in the market. }\end{array}$ \\
\hline
\end{tabular}

\footnotetext{
${ }^{1}$ Within the general framework of functions of health systems (Table 1 in the main article), financing has three subfunctions: revenue collection, fund pooling, purchasing. However, for the purpose of assessment of integration a practice model, those subdivisions were not necessary. Therefore, areas are identified for financing function in general.
} 


\begin{tabular}{|c|c|}
\hline & $\begin{array}{l}\text { Different phases of the implementation can match with different financing } \\
\text { systems better. For example, countries with social health insurance can be a } \\
\text { better place for pilot and implementation while generating evidence (11. } \\
\text { M echanisms related to evaluation of new applications and decision making in } \\
\text { health care services), because different social health insurance schemes can } \\
\text { have different ideas for implementation, increasing the chances of implemen- } \\
\text { tation. On the other hand, once evidence is generated on health gains and } \\
\text { costs and cost effectiveness is demonstrated, single payer national systems } \\
\text { can be a better environment for wide scale implementation. } \\
\text { Another question is from prevention perspective. An important incentive for } \\
\text { preventive interventions is that the investment today can save significant } \\
\text { costs in the future. However, preventive measures usually have their effect } \\
\text { (in terms of both health outcomes and cost savings) in long time spans. So, it } \\
\text { is important to know who the (third party) payer is and who will benefit from } \\
\text { the long term cost savings gained from prevention of diseases and/or compli- } \\
\text { cations. What is the time span, horizon of the payer? For example, health in- } \\
\text { surance companies in the Netherlands seem to have a short span (possibly 3- } \\
5 \text { years), because they don't know if the person who is in their policy scheme } \\
\text { will be there after } 3 \text { years. They might be reluctant to invest in services such } \\
\text { as this practice model considering that the cost savings from the prevention } \\
\text { would happen in the longer term which is not in their horizon. }\end{array}$ \\
\hline $\begin{array}{l}\text { Preliminary } \\
\text { recommen- } \\
\text { dations }\end{array}$ & $\begin{array}{l}\text { For integration of the practice model } \\
\text { - It can be considered that different phases of evidence generation and imple- } \\
\text { mentation can be carried out in different countries, based on their ad- } \\
\text { vantages and disadvantages. } \\
\text { - Countries where third party payer for primary care services would be the } \\
\text { benefiter in the time span of the return of the investment should be pre- } \\
\text { ferred. } \\
\text { To improve countries' readiness: } \\
\text { - Preventive interventions provide a 'return on investment' in the long term. } \\
\text { This may lead to inertia in countries with a SHI system for introduction of per- } \\
\text { sonal health promotion and disease prevention services, because the finan- } \\
\text { cial benefits of these interventions might take place later than the desired pe- } \\
\text { riod by SHI funders. }\end{array}$ \\
\hline \multicolumn{2}{|c|}{$\begin{array}{l}\text { 13. General health expenditure structure of the coun } \\
\text { The general picture of health expenditures of the country is required. }\end{array}$} \\
\hline $\begin{array}{l}\text { Criteria and } \\
\text { issues to be } \\
\text { considered }\end{array}$ & $\begin{array}{l}\text { - Size and distribution of health expenditure, such as per capita health expendi- } \\
\text { ture, proportion of public expenditure in overall expenditures, if data availa- } \\
\text { ble, expenditure on preventive measures in health care, in particular on com- } \\
\text { plex diseases }\end{array}$ \\
\hline Comments & - \\
\hline
\end{tabular}




\begin{tabular}{|c|c|}
\hline and examples & $\begin{array}{l}\text { - In general in the whole Europe, health care costs are rising and systems are } \\
\text { looking for ways to reduce health care costs. The financial crisis has made it } \\
\text { even worse; "Health systems require predictable sources of revenue with } \\
\text { which to plan investment, determine budgets and purchase goods and ser- } \\
\text { vices. Sudden interruptions to public revenue streams can make it difficult to } \\
\text { maintain necessary levels of health care." [50] } \\
\text { - In particular, austerity policies in response to financial crises can be a hinder- } \\
\text { ing factor for new investments. Countries implementing strict monetary poli- } \\
\text { cies, especially ones that are subject to loans of the Troika, are having budget } \\
\text { cuts for even regular health services. It might be almost impossible to make a } \\
\text { new investment such as the practice model. } \\
\text { Countries with higher public expenditure can be a better option because, in } \\
\text { principle, public funders can have a longer time span for expecting the return } \\
\text { of the investments than private funders (see also } 12 \text {. Health financing system } \\
\text { of the country) } \\
\text { In addition, the non-health benefits of implementation of new preventive } \\
\text { measures can be more appreciated within the publicly funded health systems } \\
\text { (increased productivity, etc.). }\end{array}$ \\
\hline $\begin{array}{l}\text { Preliminary } \\
\text { recommen- } \\
\text { dations }\end{array}$ & $\begin{array}{l}\text { For integration of the practice model: } \\
\text { - Countries with short and medium term budget cuts due to the financial crises } \\
\text { would have difficulty for making new investments in health care. Therefore, } \\
\text { those countries are not good candidates for integration of this practice mod- } \\
\text { el. } \\
\text { - Countries with dominant public health expenditure might be preferred. }\end{array}$ \\
\hline \multicolumn{2}{|c|}{$\begin{array}{l}\text { 14. Payment mechanisms for primary care services, i.e. GPs } \\
\text { Payment systems have an important impact on the behavior of primary care doctors [51]. The } \\
\text { payment mechanisms can have an important effect on acceptance and/or applicability new appli- } \\
\text { cations which target prevention in primary care services. }\end{array}$} \\
\hline $\begin{array}{l}\text { Criteria and } \\
\text { issues to be } \\
\text { considered }\end{array}$ & $\begin{array}{l}\text { - The payment mechanism for primary care services, i.e. GPs } \\
\text { - Underlying principles for payment, i.e. fee-for-service, capitation, fee-for- } \\
\text { performance }\end{array}$ \\
\hline $\begin{array}{l}\text { Comments } \\
\text { and examples }\end{array}$ & $\begin{array}{l}\text { - } \\
\text { - If the system is process and output oriented, the payment for prevention is } \\
\text { difficult, because what is most important in prevention is not the activity it- } \\
\text { self, but its outcome. Therefore, outcome oriented health systems, especially } \\
\text { in primary care services, would invest more in preventive applications. (see } \\
\text { also 17. Performance assessment in primary care services) } \\
\text { - If the payment principle is mainly 'fee-for-service', the practice model is less } \\
\text { likely to be accepted by the GPs. If it is a 'fee-for-performance' system, espe- } \\
\text { cially where the performance is indicated by outcome measures, instead of } \\
\text { outputs and processes, it is more likely for GPs to accept and adopt the prac- }\end{array}$ \\
\hline
\end{tabular}




\begin{tabular}{|l|l|}
\hline & $\begin{array}{l}\text { tice model. } \\
\text { - }\end{array}$ \\
& $\begin{array}{l}\text { As an example, the UK has a payment system where performance related } \\
\text { payment play an important role and GPs are incentivized to maintain the } \\
\text { health of the individuals and a number of performance criteria are used. On } \\
\text { the other hand, in the Netherlands, there is a mixture of capitation fee per } \\
\text { registered patient and fee-for-service [52]. It can be speculated that the GPs } \\
\text { would be more willing to adopt a practice model on prevention when the } \\
\text { model is linked with their performance criteria, such as the example of the } \\
\text { UK. On the other hand, this wouldn't be the case for GPs in the Netherlands } \\
\text { who are already overloaded with patient care in their practices. (see also 17. } \\
\begin{array}{l}\text { Performance assessment in primary care services) } \\
\text { Preliminary } \\
\text { recommen- } \\
\text { dations }\end{array}\end{array} \quad \begin{array}{l}\text { For integration of the practice model: } \\
\text { Countries where GPs are paid (also) for performance, rather than only ser- } \\
\text { vices should be preferred. }\end{array}$ \\
\hline
\end{tabular}

\section{HEALTH SERVICES (Primary care services) ${ }^{2}$}

\section{Strength and place of primary care services in the health system and its relationship with other levels of care}

The beneficial effects of the practice model would be most prominent in systems where the primary care physician has the function to manage the health and risks of individuals registered to his/her practice. The GP can assume this function only if he/she has a 'gate keeper' role. If the primary care services are strong and the GPs actually have a 'gate keeper' position which regulates access to specialized care, then implementation of the practice model would yield best results. If there is no gate keeping role of the GP, this means that the primary care cannot have the function to manage the health and risks of individuals and, therefore, application of the practice model wouldn't yield expected benefits. Therefore, there is a need for strong primary care system for the follow-up program to be successfully implemented.

\begin{tabular}{|l|ll|}
\hline $\begin{array}{l}\text { Criteria and } \\
\text { issues to be } \\
\text { considered }\end{array}$ & $\begin{array}{l}\text { - The position and strength of primary care services within the health system } \\
\text { - }\end{array}$ & $\begin{array}{l}\text { Health care budget spent on primary care services } \\
\text { - }\end{array}$ \\
\hline $\begin{array}{l}\text { Comments } \\
\text { and examples }\end{array}$ & $\begin{array}{l}\text { Relationship of primary care services with the whole spectrum of health } \\
\text { services }\end{array}$ \\
& $\begin{array}{l}\text { Each target country should be considered individually. } \\
\text { In countries such as the UK, where the primary care has the actual gate } \\
\text { keeping role, the practice model could play a central role on management of } \\
\text { the health, risks, and diseases of the individuals. The report generated and }\end{array}$ \\
\hline
\end{tabular}

${ }^{2}$ In the general framework of functions of health systems, health services cover a range of services (see Table 1 of the main article). Since this practice model is envisaged to be implemented in primary care services, health services heading practically cover primary care services. 


\begin{tabular}{|c|c|}
\hline & $\begin{array}{l}\text { updated by the practice model can be a crucial part of the health records of } \\
\text { the individual, which may allow the GP to manage the health of the individual } \\
\text { effectively and efficiently. } \\
\text { - If a country has a weak primary care, such as Spain, the benefits of the prac- } \\
\text { tice model would be minimal, because the follow-up would not be effective, } \\
\text { leading to diminished success in terms of the outcomes (health gains) of the } \\
\text { model. } \\
\text { On the other hand, if there are plans for strengthening or restructuring the } \\
\text { primary care services within a health reform program, it can be considered as } \\
\text { a good opportunity to structure the GP's services in a way that includes the } \\
\text { practice model. } \\
\text { If the primary care system has good links with other levels of care, i.e. special- } \\
\text { ized care, the effectiveness of the system would be enhanced. In the ideal } \\
\text { system, the reports of the practice model should follow the patient from pri- } \\
\text { mary care to other settings. Therefore, it is also important if the patient rec- } \\
\text { ords already have a continuum from primary care to specialized services and } \\
\text { other settings of health system. (see also } 22 \text {. Information flow and infor- } \\
\text { mation systems) }\end{array}$ \\
\hline $\begin{array}{l}\text { Preliminary } \\
\text { recommen- } \\
\text { dations }\end{array}$ & $\begin{array}{l}\text { For integration of the practice model and improving countries' readiness: } \\
\text { - The success of this application model lies in being implemented in a strong } \\
\text { primary care service system. Therefore, a strong primary care service struc- } \\
\text { ture is one of the prerequisites for implementation of this model. } \\
\text { - Countries with weak primary care services wouldn't be a suitable place for } \\
\text { implementation, unless it is considered to be embedded within a program on } \\
\text { restructuring and strengthening of primary care services. }\end{array}$ \\
\hline
\end{tabular}

\section{Scope of primary care in terms of (primary) prevention}

Although traditionally primary care services have prevention, including primary prevention, within their scope, the practice might be different due to several factors (see also 14. Payment mechanisms for primary care services, i.e. GPs, 15 . Strength and place of primary care services in the health system and its relationship with other levels of care, 17. Performance assessment in primary care services)

In many countries, primary prevention is in the scope of both population based services (also referred as 'public health' in the sense of health services provided to the public in general, disambiguation addressed in Chapter 2 ) and primary care services. However, in different countries, these two layers have different roles and different weights in terms of prevention of complex diseases.

\begin{tabular}{l|ll}
$\begin{array}{l}\text { Criteria and } \\
\text { issues to be } \\
\text { considered }\end{array}$ & $\begin{array}{l}\text { The primary care service provider's focus and attitudes towards prevention, } \\
\text { in particular, primary prevention }\end{array}$ \\
$\begin{array}{l}\text { Strength of the relationship between primary care services and population } \\
\text { based services }\end{array}$ \\
$\begin{array}{l}\text { If primary care have a mandate to improve the health of the people in their } \\
\text { areas or if their tasks are limited to diagnostic and curative services (scope of }\end{array}$
\end{tabular}




\begin{tabular}{|c|c|}
\hline & $\begin{array}{l}\text { the doctor) } \\
\text { - Presence of examples of risk assessment and disease prevention practices in } \\
\text { primary care, such as CV risk assessment and prevention programs (see also } \\
\text { 18. Service delivery in primary care services) }\end{array}$ \\
\hline $\begin{array}{l}\text { Comments } \\
\text { and examples }\end{array}$ & $\begin{array}{l}\text { - In the Netherlands, the scope of GP premises start from secondary preven- } \\
\text { tion and get more intense on diagnostic and curative services and tertiary } \\
\text { prevention. However, activities related to primary prevention are not gener- } \\
\text { ally in the scope of GPs. This perception likely has its roots at the perfor- } \\
\text { mance assessment and payment system of the GPs (see also 14. Payment } \\
\text { mechanisms for primary care services, i.e. GPs, 15. Strength and place of pri- } \\
\text { mary care services in the health system and its relationship with other levels } \\
\text { of care, 17. Performance assessment in primary care services). On the other } \\
\text { hand, in the UK, the role of GP for primary prevention is more clear and visi- } \\
\text { ble. } \\
\text { In parallel to this perception, primary care service providers' attitude towards } \\
\text { prevention of complex diseases might be different. For example, it seems that } \\
\text { the GPs in the UK have a proactive attitude towards prevention, inviting peo- } \\
\text { ple in their area for CV risk assessment and smoking cessation advice proac- } \\
\text { tively. } \\
\text { In the Netherlands, CV risk screening program was introduced within GP } \\
\text { premises in the last decade. These settings employ a practice nurse for man- } \\
\text { agement of chronic diseases and they also carry out the CV risk screening } \\
\text { program under supervision of the GP. The majority (82\%) of the Dutch popu- } \\
\text { lation as well as } 94 \% \text { of GPs consider general practice to be the preferred lo- } \\
\text { cation for cardiovascular risk detection [53, 54]. However, this acceptance } \\
\text { level might not hold true for primary prevention that involve lifestyle inter- } \\
\text { ventions. } \\
\text { In addition, the relationship of the primary care with population based ser- } \\
\text { vices seems to be weak in the Netherlands, whereas stronger in the UK. } \\
\text { Each target country should be considered individually. Data may not be } \\
\text { readily available on this issue for the target countries. It would be preferable } \\
\text { to conduct a separate study, but a literature review may also give some indi- } \\
\text { cation. }\end{array}$ \\
\hline $\begin{array}{l}\text { Preliminary } \\
\text { recommen- } \\
\text { dations }\end{array}$ & $\begin{array}{l}\text { For integration of the practice model and to improve countries' readiness: } \\
\text { - The practice model can more easily be implemented in countries where the } \\
\text { primary care practitioners consider prevention, in particular primary preven- } \\
\text { tion in the scope of their practice. } \\
\text { - The role of primary care services in prevention of complex diseases must be } \\
\text { strengthened, in particular in terms of personal health promotion and per- } \\
\text { sonal disease prevention services. } \\
\text { - If primary care services don't have a strong focus on (primary) prevention, } \\
\text { integration of this practice model can be used as an opportunity to expand }\end{array}$ \\
\hline
\end{tabular}


the scope of primary care services towards primary prevention.

\section{Performance assessment in primary care services}

Health systems and, as a part of it, primary care services can be oriented towards processes and outputs, which means 'doing more procedures' is rewarded, or outcomes, which means 'delivering better outcomes' is rewarded. This orientation can be visible in (presence of) the performance assessment system (if there is any).

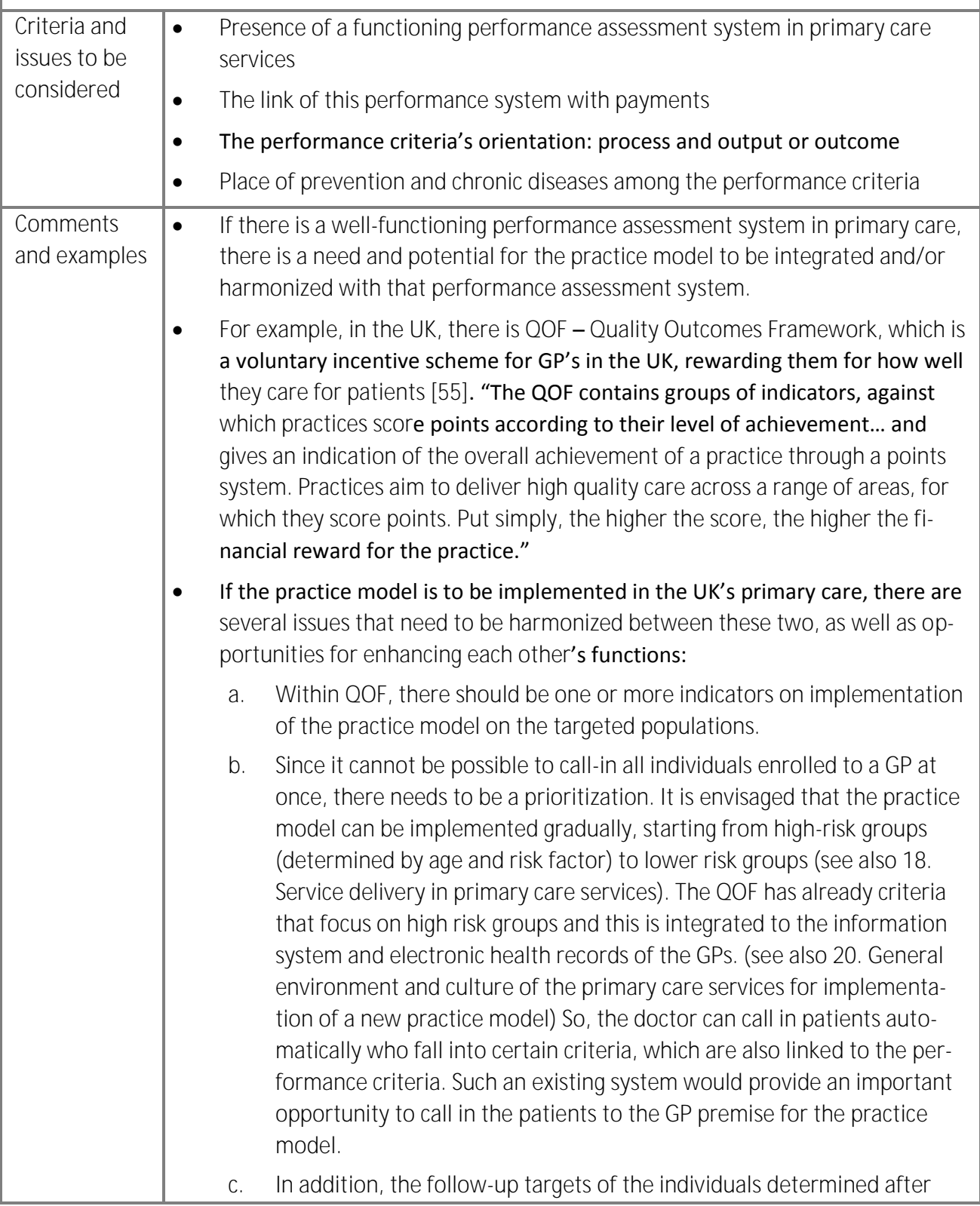




\begin{tabular}{|c|c|}
\hline & $\begin{array}{l}\text { the implementation of the practice model can be integrated in the per- } \\
\text { formance assessment system, which will incentivize reaching the tar- } \\
\text { gets. }\end{array}$ \\
\hline $\begin{array}{l}\text { Preliminary } \\
\text { recommen- } \\
\text { dations }\end{array}$ & $\begin{array}{l}\text { For integration of the practice model: } \\
\text { - Presence of a performance assessment system that is linked with the patient } \\
\text { records provides an important opportunity for implementation of the prac- } \\
\text { tice model. } \\
\text { - The practice model and the performance assessment system should be har- } \\
\text { monized with each other for optimum functioning and creating incentives to } \\
\text { reach results. }\end{array}$ \\
\hline
\end{tabular}

\section{Service delivery in primary care services}

The practice model is a personal health promotion and disease prevention intervention that is planned to be applied in wide populations. There is no early example of this yet: a comprehensive model covering primary, secondary and tertiary prevention in several health areas, which is an individual level intervention but can target the whole population. (See also 9. General environment and culture of the health system for innovation)

$\mathrm{CV}$ risk assessment programs that are being implemented recently, and which mainly focus on the people who might be in risk groups or have certain conditions (e.g. hypertension or hypercholesterolemia) is an example of a high risk approach. Although it doesn't exactly match the practice model, since there are no examples, the service delivery model can be considered somewhat similar to CV risk assessment programs, but more comprehensive.

\begin{tabular}{|c|c|}
\hline $\begin{array}{l}\text { Criteria and } \\
\text { issues to be } \\
\text { considered }\end{array}$ & 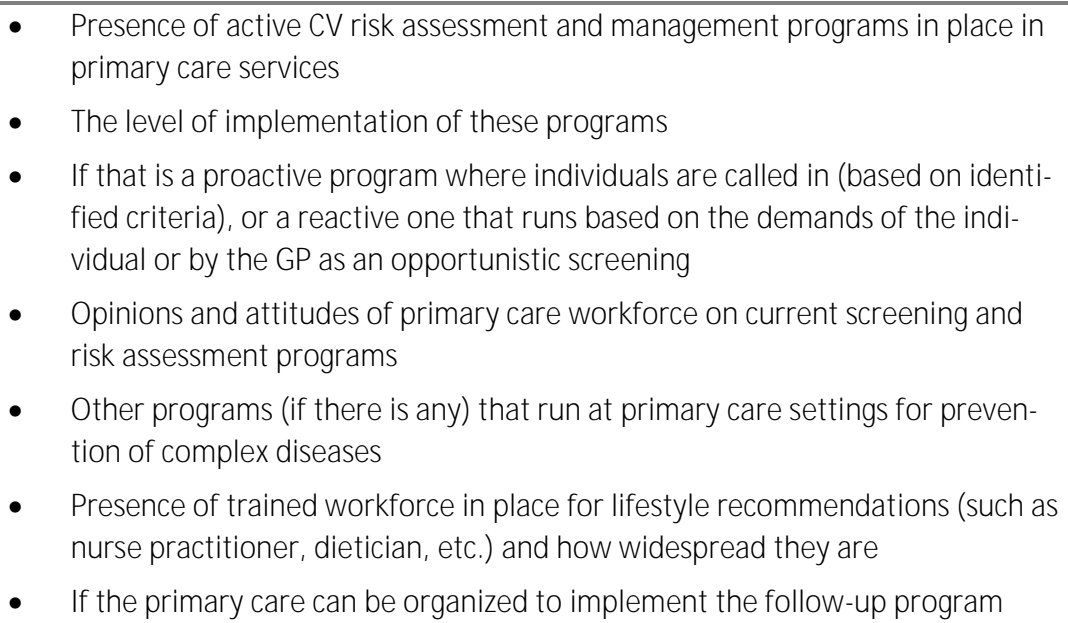 \\
\hline $\begin{array}{l}\text { Comments } \\
\text { and examples }\end{array}$ & $\begin{array}{l}\text { - } \\
\text { - } \quad \text { As an example, the CV risk assessment program in the UK is a part of the GP } \\
\text { services, where people are called in proactively by the GP's team based on } \\
\text { their risk profile (identified from the electronic health records) for risk as- } \\
\text { sessments by the nurse practitioner. The CV risk assessment is a part of the } \\
\text { performance assessment program (17. Performance assessment in primary }\end{array}$ \\
\hline
\end{tabular}




\begin{tabular}{|c|c|}
\hline & $\begin{array}{l}\text { care services) and brings a financial reward. The lifestyle advice is given by } \\
\text { the nurse practitioner and in some GP premises, dieticians are also employed. } \\
\text { - On the other hand, some GPs have a critical approach to screening programs } \\
\text { that run in GP level [56]. } \\
\text { - In the Netherlands, the CV risk assessment program is newly introduced. It is } \\
\text { not a proactive one yet, only individuals who demand it can receive it, and al- } \\
\text { so, possibly the individuals referred by the GP. The risk assessment program } \\
\text { is remunerated as a 'fee-for-service'. }\end{array}$ \\
\hline $\begin{array}{l}\text { Preliminary } \\
\text { recommen- } \\
\text { dations }\end{array}$ & $\begin{array}{l}\text { For integration of the practice model: } \\
\text { - The practice model can be introduced to the primary care services as a new } \\
\text { program and in time, it must become an integral part of the primary care } \\
\text { practice to help the GP and other professionals manage the health, risk fac- } \\
\text { tors and diseases in the context of main chronic complex diseases of the indi- } \\
\text { viduals enrolled to their practices. If there are existing CV assessment practic- } \\
\text { es, it can facilitate implementation of the practice model. } \\
\text { - There should be a system to invite people in to the program on implementa- } \\
\text { tion of the practice model. To be able to manage the resource requirements, } \\
\text { target population must gradually be expanded starting from high-risk groups } \\
\text { (determined by age and risk factor) to lower risk groups }\end{array}$ \\
\hline
\end{tabular}

\section{RESOURCE GENERATION}

\section{Health workforce}

\section{Health workforce in primary care services}

The human interaction is crucial in order to effectively deliver the health care and make it 'personalized' to the individual. However, human workforce is expensive. Therefore, a balance needs to be sought.

The health workforce related functions of the practice model is as follows:

a. Explain the individuals the scope, process, result and the limitations to create realistic expectations and avoid undue anxiety

b. Assistance in signing of the informed consent (the type of health professional can change based on the informed consent requirements of the country, see also 5 . Regulations on ethical issues in terms of implementation)

c. Assistance in filling in the (online) questionnaire and answer possible questions

d. Answer questions on current situation findings to the individual

e. Explain the risk assessment graphics to the individual

f. Explain the lifestyle program to the individual, make revisions if and when necessary and set targets with him/her for the follow-up period

g. Explain the medical follow-up program with the individual and make revisions if and when necessary

h. Carry out the follow-up program on lifestyle factors, using different methods such as coach- 
ing, goal setting, motivational interviewing, etc.

i. Carry out the medical follow-up program (integration to the usual practice of GP premise)

These functions can be covered by various professionals in primary care services, depending on the country setting.

\begin{tabular}{|c|c|}
\hline $\begin{array}{l}\text { Criteria and } \\
\text { issues to be } \\
\text { considered }\end{array}$ & $\begin{array}{l}\text { - The existing composition of health professionals that are present or can be } \\
\text { hired at GP premises } \\
\text { - } \quad \text { Presence of culture of multidisciplinary teams in primary care services, in } \\
\text { particular, role of nurse practitioners. } \\
\text { - } \quad \text { Opportunities to incorporate dieticians and other personnel on lifestyle } \\
\text { modification } \\
\text { - Baseline knowledge and skills of the primary care team on the issues listed } \\
\text { above } \\
\text { - How the above listed tasks can be distributed to the present workforce in GP } \\
\text { premises } \\
\text { If there are any functions that are not met with the present system or can be } \\
\text { met with a cheaper workforce type that is not currently present in the health } \\
\text { system } \\
\text { The presence of in-service training platforms for GPs, practice nurses and } \\
\text { other professionals in primary care services }\end{array}$ \\
\hline $\begin{array}{l}\text { Comments } \\
\text { and examples }\end{array}$ & $\begin{array}{l}\text { - In most primary care systems, GPs function together with practice nurses. } \\
\text { However, the rest of the composition can change in different target coun- } \\
\text { tries. } \\
\text { For example, nurse practitioners run the CV risk assessment programs in the } \\
\text { UK and the Netherlands. GP premises can employ dieticians in both countries, } \\
\text { but it would be questionable, and must be investigated, what percentage of } \\
\text { GP premises employ a dietician. } \\
\text { It must be investigated how the listed tasks can be distributed to the present } \\
\text { workforce in GP premises and the required human resources in terms of } \\
\text { numbers (assumptions can be made). It can be estimated that the practice } \\
\text { model will create a serious workload in GP premises and the overall health } \\
\text { system in the early years. On the other hand, if the practice model becomes } \\
\text { successful in prevention of complex diseases and help reduce their burden, in } \\
\text { the medium and longer term it will have a favorable impact on the need for } \\
\text { health workforce. } \\
\text { There is a shortage of health workforce in Europe, especially nurses, and as } \\
\text { the population is getting older, the shortage will increase because the num- } \\
\text { ber of new graduates to be employed in health sector is reducing, whereas } \\
\text { the number of old people to be cared for are increasing. Therefore, creative } \\
\text { solutions need to be found for meeting the health workforce requirements of } \\
\text { the practice model. }\end{array}$ \\
\hline
\end{tabular}




\begin{tabular}{l|l}
$\begin{array}{l}\text { Preliminary } \\
\text { recommen- } \\
\text { dations }\end{array}$ & $\begin{array}{l}\text { For integration of the practice model: } \\
\text { - }\end{array}$ \\
$\begin{array}{l}\text { With additional training, present primary care teams can probably meet the } \\
\text { functions required for implementation of the practice model. However, the } \\
\text { quantity of the workforce required can be difficult to be met. }\end{array}$ \\
$\begin{array}{l}\text { As a solution, human interaction should be kept at crucial points where the } \\
\text { presence of the health professional would make a real difference for further } \\
\text { personalization of the model, rather than repeating all what is written in the } \\
\text { report. } \\
\text { Individuals who will be enrolled to the program can attend group trainings to } \\
\text { get general information on the process, results and limitations. }\end{array}$
\end{tabular}

\section{General environment and culture of the primary care services for im- plementation of a new practice model}

Various factors may play a role on GPs attitude towards new practices in the health system.

\begin{tabular}{|c|c|}
\hline $\begin{array}{l}\text { Criteria and } \\
\text { issues to be } \\
\text { considered }\end{array}$ & $\begin{array}{l}\text { - Job satisfaction levels of the primary care team members, in particular GPs } \\
\text { and nurse practitioners } \\
\text { - Current workload of GPs } \\
\text { - } \quad \text { Opinions and attitudes of GPs to new applications, in particular their attitudes } \\
\text { towards health authorities and decision makers } \\
\text { - Professional liability insurances and possible effects of the practice model on } \\
\text { them }\end{array}$ \\
\hline $\begin{array}{l}\text { Comments } \\
\text { and examples }\end{array}$ & $\begin{array}{l}\text { - In the Netherlands, there is a general perception among GPs that after the } \\
\text { introduction of the Dutch Health Insurance Reform, their workload became } \\
\text { very high [57, 58]. Any additional task in their job description might cause re- } \\
\text { actions since they already feel overloaded and overwhelmed. } \\
\text { - GPs in the UK who might be reactive towards the screening programs that } \\
\text { run in primary care [56]. (see also 18. Service delivery in primary care ser- } \\
\text { vices) } \\
\text { - If the country has professional liability insurance schemes, the practice of the } \\
\text { model in primary care services may have an impact on the scope and premi- } \\
\text { ums of the insurance scheme. Such effects may influence the willingness of } \\
\text { the GPs to adopt the model in their practices. }\end{array}$ \\
\hline $\begin{array}{l}\text { Preliminary } \\
\text { recommen- } \\
\text { dations }\end{array}$ & $\begin{array}{l}\text { For integration of the practice model: } \\
\text { - The practice model should be implemented in a way that doesn't increase the } \\
\text { work load of the GP (and also the other primary care team members) and/or } \\
\text { remunerate the additional labor required. } \\
\text { To improve countries' readiness: } \\
\text { - Job satisfaction of primary care workforce is important for every country } \\
\text { since it might affect the openness of the workforce to innovations and devel- } \\
\text { opments in the health system, as well as their specific practices. }\end{array}$ \\
\hline
\end{tabular}




\section{Knowledge}

\section{Education and training of the health workforce}

Information contained in the report of the practice model should follow the individual in all relevant layers and settings of the health services. Therefore, all health professionals, including health service managers will need some sort of training on the practice model so that they can understand the consequences of the report. This training must be tailored to the baseline level of the health workforce. Therefore, current knowledge of the workforce is important.

Education of the new cohorts of physicians, nurses and other personnel is crucial. The implementation of the practice model should be gradually introduced to the population, starting from high risk and older groups going to younger age groups gradually, which will probably take 10-15 years' time. The education of new cohorts of health professionals will help meeting the need for more trained workforce in the future years.

As new technologies such as next generation sequencing enters into health services, such technologies can also be incorporated to the practice model. The human workforce requirement for that future should also be considered.

\begin{tabular}{|c|c|}
\hline $\begin{array}{l}\text { Criteria and } \\
\text { issues to be } \\
\text { considered }\end{array}$ & $\begin{array}{l}\text { - The profile of the current primary care team and their level of knowledge on } \\
\text { the role of genetics in complex diseases, risk assessment approaches, lifestyle } \\
\text { factors, skills that are required for assisting individuals in lifestyle changes, } \\
\text { such as coaching, motivational interviewing, etc. } \\
\text { - The place of the above mentioned areas in the curricula of the health profes- } \\
\text { sionals } \\
\text { - The training needs for primary care team, specialists and health managers } \\
\text { and other support personnel } \\
\text { - Human workforce requirement for the future of genome-based technologies }\end{array}$ \\
\hline $\begin{array}{l}\text { Comments } \\
\text { and examples }\end{array}$ & $\begin{array}{l}\text { - In the UK, a report by PHG Foundation lays out the importance and responsi- } \\
\text { bilities of bioinformaticians for services related to next generation sequencing } \\
\text { [59]. } \\
\text { - Education and training curricula will depend on the planned roles for differ- } \\
\text { ent professionals. For example, GPs might be in charge of medical aspects, } \\
\text { whereas nurse practitioners may focus on lifestyle aspects. }\end{array}$ \\
\hline $\begin{array}{l}\text { Preliminary } \\
\text { recommen- } \\
\text { dations }\end{array}$ & $\begin{array}{l}\text { For integration of the practice model and to improve countries' readiness: } \\
\text { - Different health systems may have different roles for the primary care team. } \\
\text { Considering an average primary care team in Europe, the following options } \\
\text { can be explored: } \\
\text { - The program manager of this practice model within the primary care setting } \\
\text { must be the practice nurse for the following reasons: The model doesn't only } \\
\text { involve people with risk factors or existing diseases. It involves individuals } \\
\text { without health problems. Therefore, the role of the doctor should be limited, } \\
\text { because involvement of the doctor 'medicalizes' the process and creates 'pa- } \\
\text { tients'. This holds a threat of turning every individual into potential 'patients'. }\end{array}$ \\
\hline
\end{tabular}




\begin{tabular}{|c|c|}
\hline & $\begin{array}{l}\text { Therefore, the nurse should be in central position, rather than the doctor, } \\
\text { during the implementation. } \\
\text { M anagement of lifestyle factors is crucial for the success of the practice } \\
\text { model. Coaching is one of the methods for this purpose. The best person to } \\
\text { do the follow-ups for lifestyle change in the current system seems to be the } \\
\text { practice nurse. Therefore, she needs to be educated and trained for that in } \\
\text { nursing schools. Or perhaps, an additional training can be given to the nurses } \\
\text { who want to do this type of work. Coaching lifestyle change is the key. } \\
\text { Considering the shortage of dieticians and their specific training for providing } \\
\text { nutritional plans for 'patients', role of dietician can be focused on people who } \\
\text { need certain dietary advice due to a disease - i.e. practicing dietetics. In this } \\
\text { case, nurse can focus on 'coaching' for lifestyle change. } \\
\text { If nurses are too expensive or not available, a new type of health care worker } \\
\text { can be identified: 'lifestyle coaches'. The education for this type of health } \\
\text { workforce can cover a technical education in higher education system. Unlike } \\
\text { dieticians, their focus should not only be nutrition, but also exercise and } \\
\text { smoking cessation. Alternatively, 'lifestyle coaching' can be a certificate pro- } \\
\text { gram for people with basic training in health related fields (such as certifica- } \\
\text { tion of 'lactation consultants'). } \\
\text { Until 'lifestyle coaches' are trained as new cohort of professionals, nurses and } \\
\text { dieticians would be the best type of professionals to run the follow-up pro- } \\
\text { gram for management of lifestyle factors. } \\
\text { Bioinformaticians are a new and important type of professionals for two } \\
\text { reasons: 1) For analysis of data collected for research and development pur- } \\
\text { poses, bioinformaticians are needed. } 2 \text { ) For new technologies such as next } \\
\text { gen sequencing, we need to understand the meaning and value of muta- } \\
\text { tions/ polymorphisms that is not defined before, also for service provision. } \\
\text { Therefore, bioinformaticians are important workforce need. (see 'Next steps } \\
\text { in the sequence' by PHG Foundation, the Uk [59]) }\end{array}$ \\
\hline & $\begin{array}{l}\text { rmation systems in primary care, in particular effective use of electronic health } \\
\text { rovide opportunities as well as challenges. }\end{array}$ \\
\hline $\begin{array}{l}\text { Criteria and } \\
\text { issues to be } \\
\text { considered }\end{array}$ & $\begin{array}{l}\text { - Established information systems in primary care, in particular effective use of } \\
\text { electronic patient/health records in primary care services } \\
\text { - } \quad \text { Integration of information systems between different layers and settings of } \\
\text { health care services, including the link betw een primary care services to hos- } \\
\text { pitals (is the data following patient?) } \\
\text { - Interoperability between the electronic patient/health records and the prac- } \\
\text { tice model } \\
\text { Possibilities on data to be securely stored and processed (also linked to } 7 . \\
\text { Regulations on data protection, privacy and confidentiality) }\end{array}$ \\
\hline
\end{tabular}




\begin{tabular}{|l|l|l|}
\hline $\begin{array}{l}\text { Comments } \\
\text { and examples }\end{array}$ & \begin{tabular}{l} 
- $\begin{array}{l}\text { Presence of electronic patient records in primary care would be an important } \\
\text { asset since the primary care team would be used to implementing such sys- } \\
\text { tems. On the other hand, the interoperability issues between the electronic } \\
\text { health record and the practice model can be a challenge. }\end{array}$ \\
\hline $\begin{array}{l}\text { Preliminary } \\
\text { recommen- } \\
\text { dations }\end{array}$
\end{tabular}$\quad \begin{array}{l}\text { For integration of the practice model and improve countries' readiness } \\
\text { - Interoperability with electronic health records must be considered when the } \\
\text { software for the practice model is being developed. }\end{array}$ \\
\hline $\begin{array}{l}\text { If there is no widespread use of electronic patient records in primary care, } \\
\text { implementation of the practice model can be used as an opportunity for in- } \\
\text { troducing it as well. }\end{array}$ \\
\hline Physical resources
\end{tabular}

\section{Logistical aspects}

Application of the practice model requires a system for collection of samples from primary care practices to be delivered to relevant medical laboratories. Physical infrastructure is needed for space and information and communication technologies in the GP premises.

\begin{tabular}{|c|c|}
\hline $\begin{array}{l}\text { Criteria and } \\
\text { issues to be } \\
\text { considered }\end{array}$ & $\begin{array}{l}\text { - Present systems for collecting samples from primary care practices and } \\
\text { sending them to laboratories } \\
\text { - Space and infrastructure (computers, online connection, etc.) available at } \\
\text { primary care settings }\end{array}$ \\
\hline $\begin{array}{l}\text { Comments } \\
\text { and examples }\end{array}$ & $\begin{array}{l}\text { - If there is an established logistics system for collection of samples from GP } \\
\text { premises and delivery to the relevant laboratories, it creates an advantage. } \\
\text { - The GP premise must be equipped with relevant infrastructure for ICT, as well } \\
\text { as an active electronic patient record system. }\end{array}$ \\
\hline $\begin{array}{l}\text { Preliminary } \\
\text { recommen- } \\
\text { dations }\end{array}$ & $\begin{array}{l}\text { For integration of the practice model and improve countries' readiness } \\
\text { - If there is an established logistics system for collection of samples from GP } \\
\text { premises and delivery to the relevant laboratories, it creates an advantage. } \\
\text { - The GP premise must be equipped with relevant infrastructure for ICT, as well } \\
\text { as an active electronic patient record system. } \\
\text { - If the GP premises don't have the required ICT infrastructure, implementation } \\
\text { of the practice model can be used as an opportunity to establish this infra- } \\
\text { structure which is required also for several other purposes in contemporary } \\
\text { practice of medicine. }\end{array}$ \\
\hline
\end{tabular}

\section{Laboratory services}

Countries have different arrangements for laboratory services in general and specific to genotyping.

In the near future, whole genome / exome sequencing can become a part of health services. Therefore, this future possibility also needs to be investigated. 


\begin{tabular}{|c|c|}
\hline $\begin{array}{l}\text { Criteria and } \\
\text { issues to be } \\
\text { considered }\end{array}$ & $\begin{array}{l}\text { - Facilities for high-throughput genotyping at local, regional or national levels, } \\
\text { which are licensed (or can be licensed) for carrying out genetic tests for medi- } \\
\text { cal purposes } \\
\text { Possibilities on infrastructure and expertise for the future scenarios, including } \\
\text { use of other levels of genome-based information (such as whole genome se- } \\
\text { quencing) }\end{array}$ \\
\hline $\begin{array}{l}\text { Comments } \\
\text { and examples }\end{array}$ & $\begin{array}{l}\text { - Each target country must be investigated for the arrangement of laboratories } \\
\text { in general and specific to genotyping facilities. Especially, the capacity of the } \\
\text { available local, regional and national laboratories that can provide the re- } \\
\text { quired high-throughput services must be investigated. These laboratories } \\
\text { must be licensed for carrying out genetic tests for medical purposes. (also } \\
\text { linked to 4. Regulation of genetic testing, including predictive testing for im- } \\
\text { plementation in primary care services). } \\
\text { - In the UK, there are preparations for using whole genome sequencing for } \\
\text { 'research' purposes for a very large number of patients (100.000), which may } \\
\text { be considered as a pilot for the possible future services [32]. The relevant re- } \\
\text { ports highlight the requirements and possibilities for implementation of next } \\
\text { generation sequencing in the UK [59]. }\end{array}$ \\
\hline $\begin{array}{l}\text { Preliminary } \\
\text { recommen- } \\
\text { dations }\end{array}$ & $\begin{array}{l}\text { For integration of the practice model } \\
\text { - Presence of functioning high throughput genotyping laboratories and possi- } \\
\text { bilities to use upcoming genome-based technologies are advantages for the } \\
\text { implementation phase. } \\
\text { - In many countries, current framework and regulations for genetic testing } \\
\text { laboratories is based on the 'diagnosis of monogenic diseases' paradigm. This } \\
\text { can create some problems in the practice of this model. If the genotyping } \\
\text { service providing laboratory is licensed for providing genetic testing services } \\
\text { for medical purposes and complying the relevant legislation, in principle no } \\
\text { legislative problem is expected. }\end{array}$ \\
\hline
\end{tabular}

\section{Abbreviations:}

CV: Cardiovascular

DTC: Direct-to-consumer

EU: European Union

GP: General practitioner / Family physician (main physicians who provide primary care services)

QOF: Quality Outcomes Framework

ICT: Information and communication technologies

NHS: National Health Services

SHI: Social health insurance

UK: United Kingdom 


\section{References}

1. Cesuroglu T, Karaca S, Erge S. A practice model for personalized healthcare with a public health genomics perspective. Personalized M edicine. 2009 2009/09/01;6(5):567-77.

2. M urray $\mathrm{C}$, Frenk J. A framework for assessing the performance of health systems. B World Health Organ. 2000;78(6):717-31.

3. Treaty of Lisbon amending the Treaty on European Union and the Treaty establishing the European Community, signed at Lisbon, 13 December 2007, 2007/C 306/01 (2007).

4. Together for Health: A Strategic Approach for the EU 2008-2013. Brussels: Commission of the European Communities; 2007.

5. Gaining health. The European Strategy for the Prevention and Control of Noncommunicable Diseases. Copenhagen: World Health Organization Regional Office for Europe; 2006.

6. Healthy Lives, Healthy People: our strategy for public health in England, 2010. HM Government. London: 2010.

7. Zimmern RL. Genomics and individuals in public health practice: are we luddites or can we meet the challenge? J Public Health (Oxf). 2011 Dec;33(4):477-82.

8. Transformatie in het sociaal domein [Transformation in the social domain]: Government of the Netherlands; 2015. Available from: http://www.rijksoverheid.nl/documenten-enpublicaties/kamerstukken/2015/04/07/kamerbrief-over-de-overgang-van-bevoegdheden-naar-degemeenten.html.

9. Savas BS, Cesuroglu T. Health for America: European Arguments for a Paradigm Shift. In: Shah A, Colombano J, editors. Learning from the World: New Ideas to Redevelop America Palgrave Macmillan; 2013. p. 167-84.

10. Building on our inheritance - Genomic technology in healthcare. A report by the Human Genomics Strategy Group. 2012.

11. Personalising Medical Care: Universite du Luxembourg; [cited 2013 01.07.2013]. Available from: http:// wwwde.uni.lu/recherche/focus_areas/personalising_medical_care.

12. Luxembourg Ministry of Health and the Personalized Medicine Consortium (PMC) of Luxembourg. Luxembourg Health Summit 2012 [cited 2013 01.07.2013]. Available from: http://www.sommetsante.lu/en/.

13. Wet op het bevolkingsonderzoek [Act on population screening]: Dutch Government; 1992. Available from: http://wetten.overheid.nl/BWBR0005699/geldigheidsdatum_18-06-2015.

14. Multidisciplinaire Richtlijn Preventief Medisch Onderzoek [Multidisciplinary Guideline for Preventive M edical Examinations]. Utrecht: Koninklijke Nederlandsche Maatschappij tot bevordering der Geneeskunst (KNMG) / The Royal Dutch Medical Association; 2013. Available from: http://knmg.artsennet.nl/Publicaties/KNM Gpublicatie/133282/M ultidisciplinaire-RichtlijnPreventief-M edisch-Onderzoek-2013.htm.

15. Doorlichten doorgelicht:gepast gebruik van health checks [Checking checked: appropriate use of health checks]. the Hague, the Netherlands: Gezondheidsraad / Health Council of the Netherlands; 2015. Available from: http://www.gezondheidsraad.nl/nl/taakwerkwijze/werkterrein/preventie/doorlichten-doorgelicht-gepast-gebruik-van-health-checks.

16. European Society of Human Genetics. Statement of the ESHG on direct-to-consumer genetic testing for health-related purposes. Eur J Hum Genet. 2010 Dec;18(12):1271-3. 
17. EASAC, FEAM. Direct-to-consumer genetic testing for health-related purposes in the European Union EASAC European Academies Science Advisory Council \& Federation of European Academies of M edicine; 2012 July 2012.

18. Soini S. Genetic testing legislation in Western Europe-a fluctuating regulatory target. J Community Genet. 2012;3:143-53.

19. Enactment of the German Federal Parliament (Bundestag) Human Genetic Examination Act (Genetic Diagnosis Act - GenDG), 374/09 (2009).

20. Western Pacific regional strategy for health systems based on the values of primary health care: World Health Organization, Western Pacific Region; 2010.

21. People at the center of health care: harmonizing mind and body, people and systems: World Health Organization South-East Asia and Western Pacific Bi-regional Publication; 2007.

22. Ban on direct-to-consumer genetic tests in Germany: PHG Foundation; [updated 28 April 200901.08.2013]. Available from: http:// www.phgfoundation.org/news/4562/.

23. Clark D. Genetic Exceptionalism and Paternalism Themes in New German Legislation: Genomics Law Report,Robinson Bradshaw \& Hinson 2009 [updated 2 September 200901.08.2013].

24. Directive 95/46/EC of the European Parliament and of the Council of 24 October 1995 on the protection of individuals with regard to the processing of personal data and on the free movement of such data, (1995).

25. Proposal for a Regulation of the European Parliament and of the Council on the protection of individuals with regard to the processing of personal data and on the free movement of such data (General Data Protection Regulation, 25.1.2012), 2012/0011 (COD) (2012).

26. Public Health: Commission proposes effective measures to better protect citizens from a wide range of cross-border health threats. - IP/11/1516 08/12/2011. Available at http://europa.eu/rapid/press-release_IP-11-1516_en.htm?locale=en, accessed on 01.08.03. [Press Release]: European Commission; $20 \overline{11}$ 01.08.2013]. Available from: http://europa.eu/rapid/pressrelease_IP-11-1516_en.htm?locale=en.

27. Dixon A, Robertson R, Appleby J, Burge P, Devlin N, Magee H. Patient Choice: How patients choose and how providers respond: the King's Fund; 2010.

28. Glinos IA, Baeten R. A Literature Review of Cross-Border Patient Mobility in the European Union. 2006 September 2006.

29. Rose G. Sick Individuals and Sick Populations. Int J Epidemiol. 1985 March 1, 1985;14(1):32-8.

30. Steering Group on Strategic Implementation Plan for the European Innovation Partnership on Active and Healthy Ageing. Stratgic Plan 2011. Available from: http://ec.europa.eu/research/innovation-union/pdf/active-healthy-ageing/steeringgroup/implementation_plan.pdf.

31. Department of Health UK. Innovation, Health and Wealth, Accelerating Adoption and Diffusion in the NHS. UK: Department of Health; 2011. 34 p.

32. Couzin-Frankel J. U.K. Unveils Plan to Sequence Whole Genomes of 100,000 Patients. Sciencelnsider. 2012.

33. Gallagher JE, Zheng W, Rong X, Miranda N, Lin Z, Dunn B, et al. Divergence in a master variator generates distinct phenotypes and transcriptional responses. Genes \& development. 2014 Feb 15;28(4):409-21. 
34. Craig P, Dieppe P, Macintyre S, Michie S, Nazareth I, Petticrew M. Developing and evaluating complex interventions: new guidance.: Medical Research Council; 2008. Available from: www.mrc.ac.uk/complexinterventionsguidance.

35. Whole system demonstrator programme: Headline findings - December 2011: Department of Health.; 2011 [updated 5 December 201115.05.2013]. Available from: https:// www.gov.uk/government/news/whole-system-demonstrator-programme-headline-findingsdecember-2011.

36. Whole System Demonstrator Programme - Headline Findings: Department of Health, UK; 2011 15.05.2013]. Available from:

https://www.gov.uk/government/uploads/system/uploads/attachment_data/file/215264/dh_1316 89.pdf.

37. Gornall J. Does telemedicine deserve the green light? Bmj. 2012 2012-07-10 11:37:18;345.

38. A concordat between the Department of Health and the telehealth and telecare industry: Department of Health; 2012 29.10.2013]. Available from: https:// www.gov.uk/government/uploads/system/uploads/attachment_data/file/216757/Concorda t-3-million-lives.pdf.

39. 3millionlives Background: 3millionlives; [29.10.2013]. Available from: http://3millionlives.co.uk/about-3ml\#background.

40. Simpson S, Hiller J, Gutierrez-Ibarluzea I, Kearney B, Norderhaug I, Fay A, et al. A toolkit for the identification and assessment of new and emerging health technologies. Birmingham: EuroScan, 2009 June 2009. Report No.

41. Lal JA, Schulte In den Baumen T, Morre SA, Brand A. Public health and valorization of genome-based technologies: a new model. J Transl Med. 2011;9:207.

42. NIHR Horizon Scanning Centre: University of Birmingham; 2013 [01.07.2013]. Available from: http://www.hsric.nihr.ac.uk/.

43. Markopoulos C. Overview of the use of Oncotype DX®as an additional treatment decision tool in early breast cancer. Expert Review of Anticancer Therapy. 2013 2013/02/01;13(2):179-94.

44. NICE. Guide to the methods of technology appraisal 2013 [Process and methods guides]: National Institute for Health and Care Excellence; 2013 18.10.2013]. Available from: http://publications.nice.org.uk/pmg9.

45. Schlander M, Affolter C, Sandmeier H, Brügger U, Cao C, Cueni T, et al. "Value \& Valuation of Health Technologies" Swiss HTA Consensus, Guiding Principles. Final Version FV, March 13, 2012 ed. Basel, Bern, Solothurn and Wiesbaden: InnoVal - Institute for Innovation \& Valuation in Health Care; 201213 March 2012.

46. Menon D, Stafinski T, Nardelli A, Jackson T, Jhamandas J. Access with evidence development: an approach to introducing promising new technologies into healthcare. Healthcare management forum / Canadian College of Health Service Executives = Forum gestion des soins de sante / College canadien des directeurs de services de sante. 2011 Summer;24(2):42-56.

47. Khoury MJ, Coates RJ, Evans JP. Evidence-based classification of recommendations on use of genomic tests in clinical practice: Dealing with insufficient evidence. Genet M ed. 2010;12(11):6803.

48. Kutzin J. Bismarck vs. Beveridge: is there increasing convergence between health financing systems? (1st annual meeting of SBO network on health expenditure, 21-22 November 2011, Paris, OECD): OECD; 2011 [01.07.2013]. Available from:

http://www.oecd.org/gov/budgeting/ 49095378.pdf. 
49. Busse R. From Bismarck and Beveridge to "Bisridge » type: developments and convergence in EU health systems (European Public Health Conference, 20 years of the Maastricht Treaty - Turning past experiences into visions, 22-23 May 2013, M aastricht). 2013.

50. M ladovsky P, Srivastava D, Cylus J, Karanikolos M, Evetovits T, Thomson S, et al., editors. Health policy responses to the financial crisis in Europe: World Health Organization \& European Observatory on Health Systems and Policies; 2012.

51. Gress S, Denoij DMJ, Groenewegen PP. Managing primary care behaviour through payment systems and financial incentives. In: Saltman RB, Rico A, Boerma W, editors. Primary care in the driver's seat -Organizational reform in European primary care. European Observatory on Health Systems and Policies series: Open University Press; 2006. p. 184-200.

52. Schäfer W, Kroneman M, Boerma W, van den Berg M, Westert G, Devillé W, et al. The Netherlands: Health system review. Health Systems in Transition. 2010;12(1):229.

53. Gelissen R, Jonkers R. Nulmeting Gezondheidscheck CMR Uitkomsten van onderzoek onder de Nederlandse bevolking van 45-74 jaar in het kader van het project Checkstandaard Cardiometabool Risico: LekkerLangLeven.nl; 2009. Available from: http:// www.lekkerlangleven.nl/l/library/download/2113.

54. Nielen MM, Assendelft WJ, Drenthen AJ, van den Hombergh P, van Dis I, Schellevis FG. Primary prevention of cardio-metabolic diseases in general practice: a Dutch survey of attitudes and working methods of general practitioners. The European journal of general practice. 2010 Sep;16(3):139-42.

55. About the Quality and Outcomes Framework (QOF): NICE - National Institute for Health and Care Excellence; 2013 [updated 27 August 201320.01.2013]. Available from: http://www.nice.org.uk/aboutnice/qof.

56. M M Cartney M. The Patient Paradox: Why Sexed Up M edicine is Bad for Your Health Pinter \& Martin Ltd.; 2012.

57. Lako $C$, van den Hombergh $P$, Honingh $M E$, Janssen P. Huisartsen over marktwerking, werkdruk en kwaliteit van zorg. Tijds gezondheidswetenschappen. 2014 2014/01/01;92(1):19-21. Dutch.

58. van de Streek J. Werkdruk en financiering staan nazorg bij huisarts in de weg. Mednet [Internet]. 201418 June 2015. Available from:

http://www.mednet.nl/wosmedia/2265/oncologie_onderzoek.pdf.

59. Wright C, Burton H, Hall A, M oorthie S, Pokorska-Bocci A, Sagoo G, et al. Next steps in the sequence. The implications of whole genome sequencing for health in the UK. Cambridge: PHG Foundation; 2011 October 2011. 
You shall not accept any information, unless you verify it for yourself. I have given you the hearing, the eyesight, and the brain, and you are responsible for using them.

Quran, Al-Isra, 36 
Chapter 8

General Discussion 


\section{Contents}

1. Interaction of health systems and personalized health care practices for effective prevention of chronic complex diseases

1.1. Setting the scene: from Health for All to 'tailored' and 'personalized' approaches 248

1.2. The need to innovate 250

1.3. Personalized health care in public health and health systems; an overview of relevant concepts 252

1.3.1. Looking forward: innovation for prevention of chronic diseases 252

1.3.2. Back to basics: primary care services for prevention of chronic diseases ... 263

1.3.3. Properties of health systems that would better facilitate the integration of a preventive personalized health care model 266

1.4. Innovations for personalized health care in light of health systems 268

1.4.1. The need for innovation for 'personalized' practices 269

1.4.2. Innovation to adapt to target countries and health systems 270

1.4.3. 'Personalized' practices in health system contexts ...................................2 275

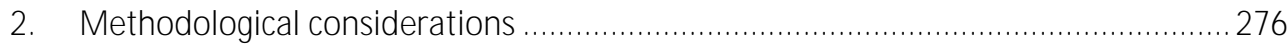

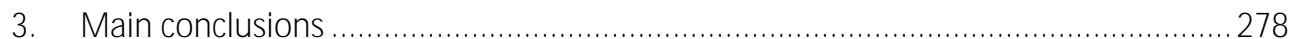

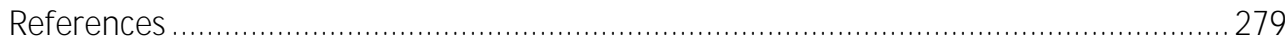


The starting point of this $\mathrm{PhD}$ research was a serious societal problem that creates a huge burden and economic toll on health systems and societies: chronic complex diseases. Driven by scientific and technological developments, personalized medicine and health care have the potential to provide solutions for effective prevention of chronic diseases. However, integration of 'personalized' practices into health services has been a challenging process [1, 2]. This dissertation addressed this challenge and took a journey through the fields of personalized medicine and health care, and health systems and policies. Gentest, a preventive personalized health care model, has been my 'vehicle' in this journey.

The aim of this dissertation was to investigate how a personalized health care model, such as Gentest, can be integrated in primary care services in European health systems, for prevention of chronic diseases. To answer that, I asked three sub-questions. 1. Where will the practice model be integrated?; 2. What will be integrated?; and 3. How can the integration take place?

Part I answered 'where' the integration will take place and described the setting, i.e. health system in its wider context of public health. Part II answered the 'what' question by first describing the broader context of personalized medicine and personalized health care and then introducing Gentest, which is an example of a practice model to be integrated into the health systems in Europe. Part III presented a systematic approach to assess 'how' this practice model can be integrated in primary care services in European health systems. It exhibited an inventory of issues that may be involved in the integration process of the practice model we took as our example.

This last chapter will take a step back and try to reflect on the interaction between the contexts of the health systems and personalized health care practices (section 1). I will discuss what adaptations are needed within the health system to successfully integrate personalized medicine and health care, as well as how personalized practices need to change in order to fit in the health systems. An additional section will reflect on our use of a concrete, real life case in this dissertation and how it may contribute to research in this field (section 2).

\section{Interaction of health systems and personalized health care practices for effective prevention of chronic complex dis- eases}

This section will first take a look at the past 40 years of public health to see very briefly how approaches to disease prevention and health promotion evolved. Following this scene setting, a case for 'co-evolution' of innovations on both health systems and personalized health care practices side will be created. Then, I will look at health system and personalized health care innovations from each other's perspective respectively. 


\subsection{Setting the scene: from Health for All to 'tailored' and 'person- alized' approaches}

When the vision of 'Health for All' (HFA) was born in the World Health Assembly in 1977 as "the attainment by all the people of the world by the year 2000 of a level of health that will permit them to lead a socially and economically productive life" [3], it breathed new life into public health discourse. As introduced briefly in Chapter 2, HFA was a revolutionary paradigm shift to bring World Health Organization (WHO) and member states from a 'combating disease' approach to one that considered health and health policy in the broadest sense.

The three words of 'Health for All' title reflect the essence of this paradigm shift. With the word 'health', HFA moved the focus from 'processes' towards 'outcomes': governments were to be held accountable for the 'health' of their citizens, not just for the 'health services' they provided [4]. Moreover, health wasn't seen as merely 'the absence of disease' anymore. It was a state of well-being physically, mentally and socially.

With the phrase 'for all', HFA addressed the value of 'equity', emphasizing that everyone had the right to attain their full health potential [5] (see also Chapter 2). Additionally, HFA clarified that it is not only the task of ministries of health or the health sector to attain this goal. 'All' sectors, including the ones other than health, had a responsibility and role to achieve HFA.

How can countries around the world achieve HFA? The primary intent of the International Conference on Primary Health Care, in Alma-Ata, in 1978, was to put forward the strategies to realize HFA across the globe. The declaration proposed 'Primary Health Care' as the model to reach HFA around the world ${ }^{1}$.

In an effort to develop the required strategies to realize the HFA vision in the European region, in 1984, WHO Europe developed and adopted the 38 targets for 'Health for All by the year 2000' [6]. This policy document made a great contribution to the spread and recognition of a broad understanding of health promotion [4]. Soon after, Ottawa Charter for Health Promotion was adopted in 1986 at an international conference held in Ottawa, Canada, under the leadership of WHO. It framed health as a 'resource for everyday life' and defined health promotion as the process of enabling people to increase control over, and to improve, their health. It emphasized "Health promotion is not just the responsibility of the health sector, but goes beyond healthy life-styles to well-being". Ottawa Charter proposed 5 key action areas that involve all levels that have an impact on the individual's health, from macro to micro: 1) building healthy public policy, 2) creating supportive envi-

\footnotetext{
1 'Primary health care' and 'primary care services' are different concepts, as summarized in Chapter 2.
} 
ronments, 3) strengthening community actions, 4) developing personal skills, and 5) reorienting health services.

In late 80 s, as a response to interventions that focus on only the 'individual' and informing them to change their lifestyle, without considering the context they live in, an 'ecological' approach started to evolve in the field of health promotion. Sole focus on individual lifestyle changes was criticized to be a 'victim-blaming ideology' because it neglected the importance of social influences on health and disease [7]. People's lifestyle is not determined only by the decisions of the individuals. It is shaped by the social, economical and physical opportunities and constraints of the environment they live in. Ecological models proposed to take this into account and assume that "appropriate changes in the social environment will produce changes in individuals, and that the support of individuals in the population is essential for implementing environmental changes."[7]

Thus, Ottawa Charter and the ecological approach concurrently brought into attention the environment (or the context) in which individuals live in as an important determinant that underlies behavior and lifestyle. They proposed to target this environment, among others, to allow for healthy behavior and positive health outcomes.

It was very important at that point in time to understand that the context of the individual is a conglomeration of interacting determinants that shapes individual behavior and lifestyle choices. The elements that constitute the environment are shared to a certain extent. However, in each individual context they may interact in slightly different ways, shaping the health environment of each individual in a unique way. In particular for interventions that aim to change the individual's behavior, this requires different communication and behavior change strategies to reach different individuals. Realization of this fact triggered moving away from the 'one-size fits all' approaches and initiated 'targeted' and 'tailored' approaches in health education and promotion in 1990s and 2000s [8]. Here, targeting means that a single intervention is developed for a specific, clearly defined target population (for example, 40-65 year old black women in the whole country or bluecollar man in a certain workplace), whereas tailoring aims to reach each person with information or change strategies based on the unique way in which that individual's characteristics and environment are interlinked.

Interestingly, late 1990s and early 2000s is also the time when the 'personalized' approaches to medicine and health care started to be discussed in the literature and scientific fora. In the earlier phase, the term 'personalized' and 'individualized' was used mostly for genome-based and information/ communication technologies (ICT) driven applications, but later these terms broadened to cover various other ones, including practices that have no technological component (see Chapter 4). Concurrent development of 'personalization' and 'tailoring' concepts in different fields points that there has possibly been a general societal trend that drove these. 
Chapter 6 introduced a personalized health care practice that aimed to prevent common chronic diseases in primary care settings. This practice model, Gentest, utilizes individuals' personal health information, detailed lifestyle analysis, body composition, genotype, and other biomarkers in order to prevent the onset or progression of major chronic diseases in a targeted way. Such comprehensive personalized health care practices have the potential to integrate different 'personalized' and 'tailored' tools into a single practice model that can be used to manage the 'health' of the individuals in a holistic way and lead to effective lifestyle changes. Nevertheless, this potential can be achieved only if the health system is ready to host them.

\subsection{The need to innovate}

In spite of the reorientation from disease to health, from cure to prevention, and from uniform to tailor-made approaches in the past 4 decades, even 15 years after the target year of 2000, we still haven't completed the paradigm shift towards HFA. A large part of our health budgets is still spent on diagnosing and curing 'diseases', rather than on promotion of 'health' and prevention of 'diseases'. Additionally, the translation of health promoting efforts into policies and actions in countries is progressing slowly. Following the footsteps of Ottawa charter, serious efforts have been spent to involve sectors other than health into health policy, in particular with the 'governance for health' concept and health in all policies [9-11]. However, in many countries around the globe, health is still mainly seen as a function/outcome of health services.

Thus, the 'old' HFA vision is not only still valid, but also still provides a coherent overarching framework for the overall goal of public health, as we've set it in Chapter 2. It would thus provide an excellent 'base' for innovative technologies and could rejuvenate old ideas concerning a holistic and context-based approach to health.

If we look at the health systems more closely, to attain HFA, we need to shift towards proactive and health oriented services focused on prevention as well as chronic cure and care, covering the whole spectrum of health care (not only medicine). There are the countless efforts to develop intervention models or tools to prevent disease and/or promote health, however, they often focus on specific diseases or specific intervention areas (such as smoking cessation, healthy nutrition and physical activity). An integrated approach to prevention ${ }^{2}$, as well as diffusion of the existing individual interventions in the health system, although presented often on paper and in policies, are still largely lacking in reality. To attain the desired paradigm shift at the system level, we need think outside the box; we need to innovate.

\footnotetext{
${ }^{2}$ i.e. an approach covering each individual from birth till death, involving the entire health spectrum of that individual, and taking the unique constellation of biological as well as lifestyle and environmental determinants of that individual into consideration
} 
In the health sector, the term 'innovation' is mostly perceived as equivalent to 'new technologies', such as development of pharmaceutical and other therapies, as well as medical devices such as diagnostics [12]. We also see this tendency in the field of personalized medicine, as raised earlier in Chapter 4. However, technical and technological innovation at the level of health interventions, such as 'personalized' practices like pharmacogenomics or tumor genomics, is not enough. They alone cannot have a profound impact on the burden of chronic diseases. It is not enough to simply release them, as the system, where they will be used, also needs to be innovated to be able to effectively adopt them.

In Chapter 4, this issue was taken more specifically, for the case of the interaction among 'personalized' innovations and the health practices. It was emphasized that innovations on the science and technology side are not enough. To realize the vision of personalization, they need to be adapted and further developed on the health practice side. At the end of the day, those practices will actually construct how personalized approaches will take place in medicine and health care, not only specific techniques or technologies.

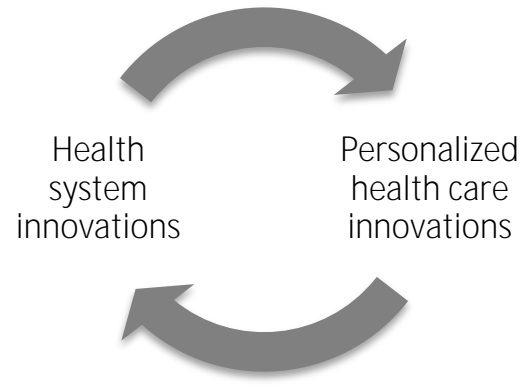

\section{Figure 1: Co-evolution of personalized health care and health system innovations}

Health systems need to innovate to better host personalized medicine and health care innovations and personalized innovations need to innovate again to adapt to the health system. This creates a cycle of co-evolution of innovation, which helps to elevate each other to a higher level to better meet the needs of the population.

If we enlarge our focus from health practices towards capturing the health system in its entirety, we see that what was set forth in Chapter 4 is also true for the interaction among the innovations in personalized health care and innovations in the health system. To innovate in the health system, we should look at the entirety of the health system as a 'complex-adaptive' system $[13,14]$. There is a need to re-think how health systems, as well as health services as a part of it, are organized and to investigate what kind of innovations are needed at the systems level to enable and host the upcoming innovative interventions, such as personalized medicine and health care, in the best possible way. If we are really aiming to achieve the paradigm shift that is required to tackle the burden of chronic diseases, we need to look at both areas, instead of focusing on only one of them. We need 
to initiate a process of co-evolution (or termed as 'co-production' in current science, technology and society studies [15]), in which 'personalized' practices adapt in order to fit to the health system, and in which health system changes to make the innovative practices of personalized medicine and health care fit. (See Figure 1)

The following two headings will look at this interaction from two perspectives. First one is looking at health systems and required innovations from the perspective of personalized medicine and health care. The other one is looking at 'personalized' practices and efforts in this field from the perspective of health systems and policies. This will be done in light of the HFA vision, since it was proposed as the overarching framework for public health in Chapter 2. As it was throughout this dissertation, Gentest, the practice model chosen as the example, will be the 'vehicle' to make this discussion effectively.

\subsection{Personalized health care in public health and health systems; an overview of relevant concepts}

The practice model introduced in Chapter 6 (Gentest) is a comprehensive and integrative model of personalized health care for prevention of common chronic diseases. It includes a wide range of tools, which, in combination, help the individual achieve a healthy lifestyle and manage his/her health according to individual health priorities.

Following its pilot in Turkey and upon being identified as a 'best practice model' by Public Health Genomics European Network (PHGEN) in 2008, it was seen that the main implementation area for such a preventive health care product would be primary care services in Europe, where it can be provided via general practitioners/ family physicians.

This sub-section will propose to revisit some of the public health concepts, including health systems, in light of the developments in the personalized medicine and health care field, in particular our practice model. I will first shortly go through a few conventional approaches in public health and prevention and question if they are ready for the upcoming practices of personalized health care. The practice model will be used as a starting point for contemplation. As the concepts are revisited, I will make proposals for alternatives to some of them (looking forward). Then, I will go back to basics to emphasize the transformation needed within primary care services for effective prevention of chronic diseases, and show how our practice model could be instrumental for that. Lastly, the answer to question that was already covered in Part III (Chapter 7) will be summarized, but this time in a more explicit way: what kind of health systems would facilitate the integration of the practice model in the best possible way?

\subsubsection{Looking forward: innovation for prevention of chronic diseases}

Chapter 1 introduced the 'disease development curve' and pleaded to focus on earlier phases of diseases development (Phase I in Figure 2-a. in this chapter) in order to prevent chronic diseases effectively. The introduction of 'personalized' practices at the early phas- 
es will have a profound effect on how we approach 'prevention' in the context of chronic diseases, as well as public health concepts.

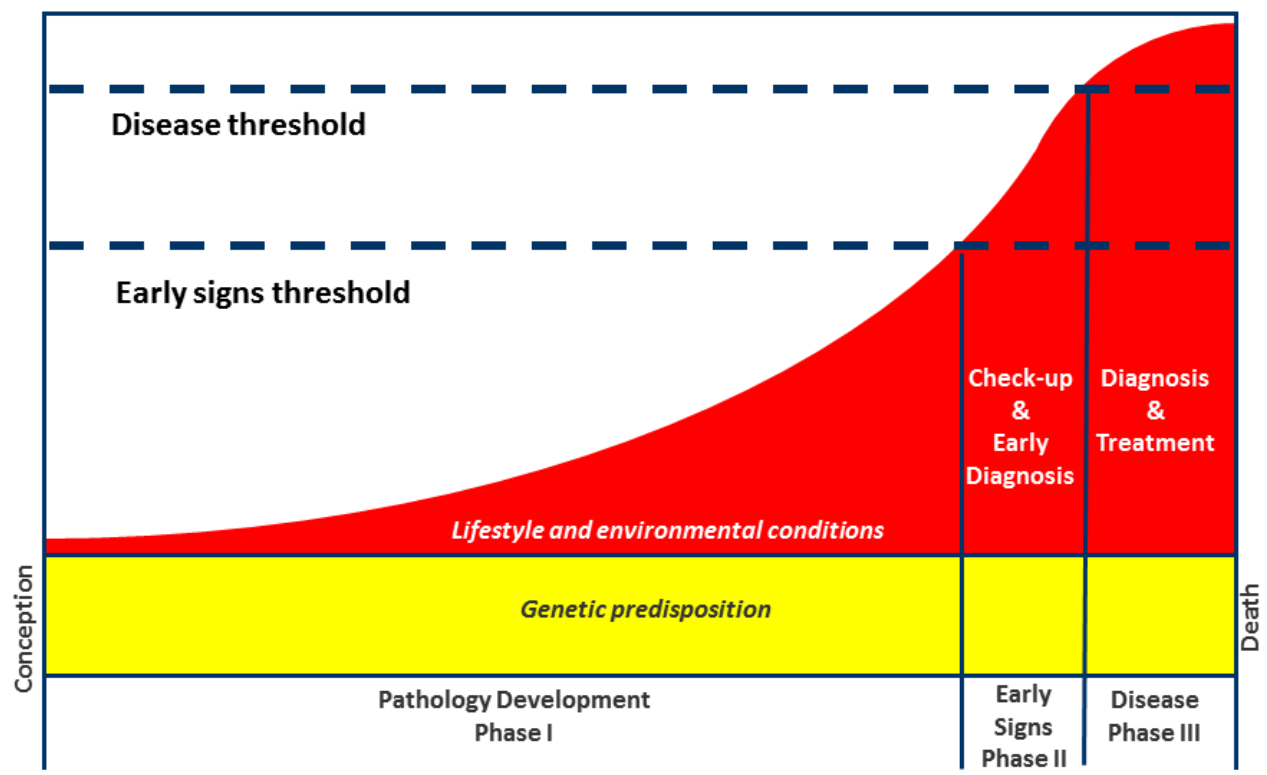

a. Disease development curve

\section{Personal health management}

Personal risk management

Personal disease

management

\section{b. Preventive approaches in different phases of the disease development}

Figure 2: 'Disease development curve' - (a) Development of chronic diseases in a time perspective and (b) preventive approaches for different phases of disease development

Source: The 'disease development curve' was developed by Serdar Savas at GENAR Institute for Public Health and Genomics Research, Ankara, Turkey, in 2008, inspired by the disease progression curve of Snyderman [16] to a certain extent. The concept of proposing health management, risk management and disease management as preventive approaches in different phases of disease development was developed by Serdar Savas for Diabetes 2020: Vision and Targets project [17].

In Chapter 7 (Part III) we identified 24 areas that should to be investigated to assess how our practice model can be integrated into a health system. One of them briefly introduced in which areas the practice model could challenge the conventional mindsets in health 
sector ('9. General environment and culture of the health system for innovation' in Supplementary Material of Chapter 7). The approach to prevention was one of them. This section will take this further and attempt to revisit some rather conventional approaches and classifications and/or fuel already existing debates.

\section{Personal vs. population based strategies for prevention}

The classical prevention approach, which dates back to Rose, 1985, suggests that there are two strategies to prevention: population based and individual. The first seeks to control the determinants of disease in the population as a whole. Population based mass interventions make small benefits for individuals, but their cumulative effect on the burden of disease on the population level is substantial. Within the second strategy, individual interventions for prevention of diseases are reserved for high-risk susceptible individuals. This strategy seeks to identify and offer high-risk individuals some protection $[18,19]$. One of the main reasons for proposing a population based approach to prevention by Rose has been that approaching each and every individually for prevention with the tools and resources available in that period would not have been possible.

While several scholars still support Rose's 'population based vs. high-risk individual' prevention strategies, there are some calls to revisit the dichotomy for a number of reasons. Firstly, identification of high-risk individuals has improved in accuracy and some believe that this obviates the need for population-wide prevention strategies [20, 21]. Additionally, the population based approach was criticized for its potential to exacerbate disparities. To prevent that, Frolich and Potvin proposed a vulnerable population approach to complement the population based ones [22]. More recently, Burton et al. proposed that another strategy would be segmenting the population by risk in to a number of strata, to each of which differential interventions may be applied. While determining the strata, genetic factors might be used. This is called 'stratified prevention' and they argue that such an approach will lead to consequential advantages in efficiency, effectiveness and harm minimization [23].

Before presenting my point about revisiting the 'population based vs. high-risk' dichotomy, it is worth to discuss what is meant by 'population based' interventions. Roughly, preventive interventions may be seen as representing two extremes of a spectrum, referred to with different terms by different authors, theories or perspectives. These are for instance "superficial" and "radical" [19, 24], "agentic" and "structural", founded on the agency-structure sociological theory [25-27], and "downstream" and "upstream", from the perspective of socioeconomic determinants of health [28, 29]. Although they are driven by different theories, there is substantial overlap, which indicates a similar division. On one hand, there are interventions that aim to change the behavior of the individual by informing him/her, relying on the capacity of individuals to make independent, purposive choices (superficial, agentic or downstream). On the other hand, there are interventions towards the structure underlying individual behavior and lifestyle. This structure then involves 
social, economic, political, and material contexts within which behavior occurs (radical, structural or upstream).

Ecological models in health promotion provide a wider perspective and claim that there are actually more levels between the individual and the whole population [7]. Rather than targeting solely the structure or solely the individual behavior, ecological models stress that there are intermediate levels in between that interact. The factors range from the biological and physiological processes taking place within the body to individual characteristics such as age, race, sex, behaviors, family, social and community networks, living and working conditions, broad social, economic, cultural, health and environmental conditions and policies. All these levels are linked, interact, and come together in the individual in variety of ways (Figure 3). Which elements play a dominant role and how they interact, however, differs from one individual to the other, requiring not only a needs and risk assessment at individual basis, but also intervention at personalized level. Holistic ecological models for health promotion, in other words, offer the potential for a tailor-made intervention at the level of the individual.

Figure 3 introduces a proposal for illustration of how individuals' biopsychosocial and ecological factors interact to explain health and health related behavior. It is based on the "Biopsychosocial ecological system" model of Sameroff [30]. The visualization of the ecological context is additionally enriched using the model of the Institute of M edicine (2003) [31], which provided an elaborated approach to multiple layers of determinants of health. Such holistic approaches towards understanding individuals' health may start a debate around them and facilitate different ways of thinking to develop holistic personalized health care practices.

Ecological models stress the importance of structural interventions at population base because they target the deep roots of the chronic diseases, such as the social, economic, political and material contexts and, therefore, can produce effective results. For example, prohibiting sales of tobacco products to people under 18, creating smoke-free environments, raising the price of tobacco products through tax and other policies, restricting/banning tobacco advertisement, countering advertising with prevention and cessation messages, and plain-packaging are among the structural interventions have been proven to be powerful methods to reduce smoking rates [32, 33]. However, such interventions are hard to achieve, since tackling those deep roots of chronic diseases require a strong commitment on the side of the politicians and decision makers. This is one of the main reasons why structural interventions in other much needed areas, such as decreasing sugar and unhealthy fat consumption and increasing physical activity, are developing at a very slow pace. Implementation of effective policies to tackle them requires a strong will on the decision makers' side since the interventions will involve agricultural policies at large and the food industry [34-37]. 


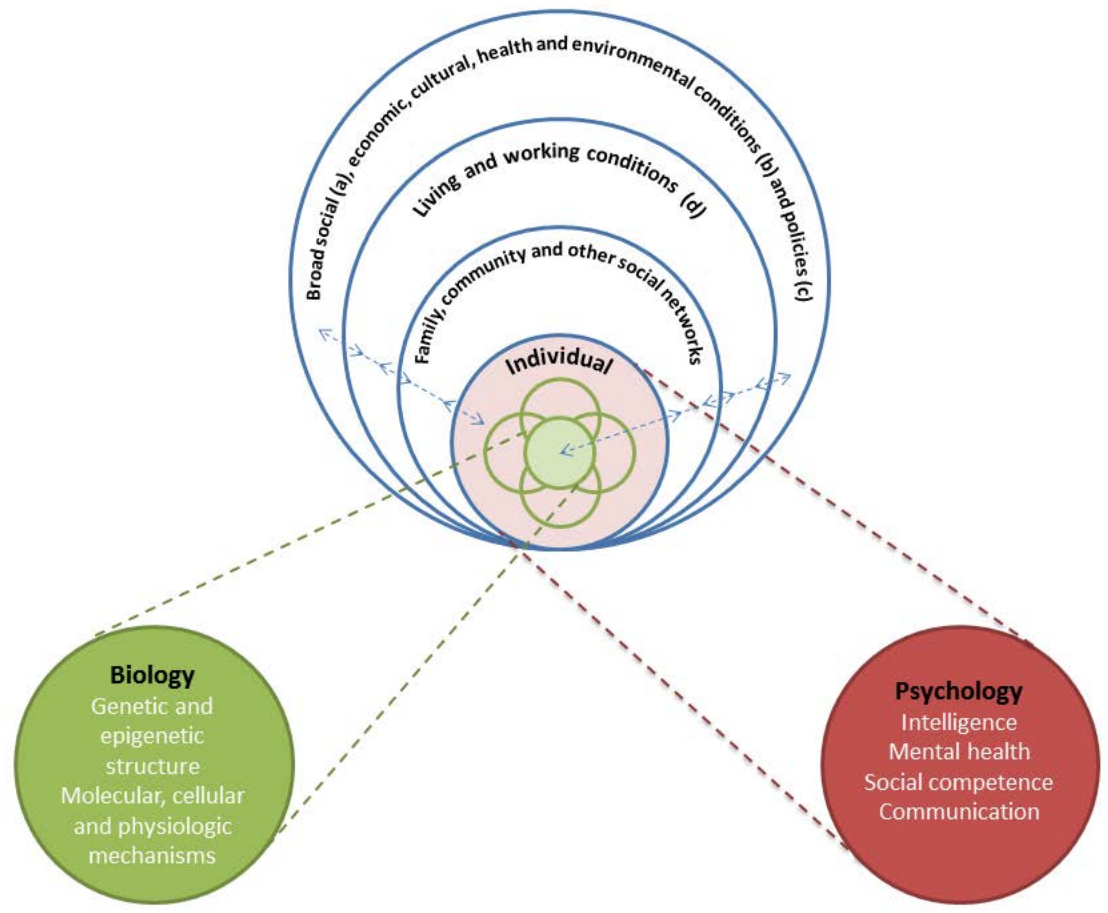

Figure 3: A proposal for conceptualization of an integrated bio-psycho-socio-ecological approach to health

Notes of the figure:

1. This is a proposal for conceptualization of an integrated bio-psycho-socio-ecological approach to health. It aims to explain health and health related behavior. For this model, Sameroff's "Biopsychosocial ecological system" model (2010) [30] and the multilayered approach to population health by Institute of Medicine (2003) [31] were integrated into a single illustration.

2. (a) Social conditions include, but are not limited to: economic inequality, urbanization, mobility, cultural values, attitudes and policies related to discrimination and intolerance on the basis of race, gender, and other differences.

(b) Other conditions at the national level might include major sociopolitical shifts, such as recession, war, and governmental collapse.

(c) Policies at the global, regional, national, state/provincial and local levels

(d) Living and working conditions may include: Psychosocial factors, employment status and occupational factors, socioeconomic status (income, education, occupation), the natural and built environments (transportation, water and sanitation, housing, and other dimensions of urban planning), and health services (population based and individual).

In the light of the complexity and slow pace of structural changes, individualized prevention, however is still needed. It is for this reason that holistic, ecological approaches propose to include these levels as well. This calls for strategies that couple structural interventions at the population level with personal level interventions that motivate and 
empower people to adopt healthy lifestyles, in particular with 'tailored' and/or 'personalized' interventions (see 1.1. Setting the scene: from Health for All to 'tailored' and 'personalized' approaches).

In the case of smoking, for example, those who have already started smoking would be more motivated by personal reasons (such as seeing the increase in cardiovascular risk visually, as well as the potential decrease that will happen when they stop smoking, or monetary savings they will gain). They would thus be more empowered by personalized recommendations based on an analysis of their underlying causes of smoking, instead of generic and usual advice of "you should not smoke because it is bad for your health".

Because of their comprehensive nature, personalized health care practices can become important tools in ecological approaches to health promotion. They provide an excellent opportunity to integrate biological characteristics (at genetic and other physiological levels), and other personal characteristics (age, gender, ethnicity, lifestyle, socioeconomic status, living and working conditions, environmental conditions, culture, etc.) into 'tailored' and/or 'personalized' tools that can be used in practice. Thus, ecological models provide the approach and tools we need in order to identify what the individual's context is like. Once the context is known, then 'personalized' or 'tailored' interventions can be developed.

If we look at our example, Gentest, we see that a main part of its mission is (see Chapter 6) to change the behavior of the individuals according to their health priorities. And, to do that, it incorporates various elements of the individual context. In this sense, it shares a similar approach with the 'tailored' health promotion interventions that are designed to address individual contexts. On the other hand, the second part of its mission is managing the health of the individual according to their health priorities. For both components of the mission, various assessments are made on the personal health history, family health history, lifestyle factors including nutrition, exercise and smoking cessation, as well as on the genetic structure of the individual. In this sense, Gentest is in line with 'personalized health care', as it has the holistic approach to health that ecological models in health promotion call for. (For a more detailed discussion on what 'personalized health care' is, see Chapter 4 and 5)

However, personalized health care does not only provide tools that can become central to ecological approaches for particular health risks (cardiovascular diseases, diabetes, cancers, osteoporosis, chronic obstructive lung diseases, etc.). Unlike many health promotion interventions, personalized health care practices, such as Gentest, can be used to monitor the health of the entire population. Moreover, it is not a programmatic approach that focuses on particular health risk or disease, or on particular high-risk populations. Instead, it can monitor our health from birth till the end of life and claims to include most of the major health threats. With the effective use of ICT and decreasing costs of genetic tech- 
nologies, the intervention can be delivered to large population clusters, not only high-risk groups. This has three implications. First of all, the individualized lifestyle recommendations can slow down the progression of the disease development curve, and thus may help to delay or prevent the development of chronic complex diseases to a certain extent in the population. Secondly, as Burton et al. raised earlier, such a model allows stratification of the individuals into several risk groups and delivering appropriate preventive interventions for each stratum, not only for high-risk strata [23]. Lastly, different strata can be approached with individual level interventions, as well as population-based interventions, depending on the intervention. Thus, personalized health care models may lead to questioning the classical 'population based vs. individual (high risk) prevention' strategy and help us approach prevention in different ways.

Targeting the whole (relevant) population with individual interventions is not a totally new concept. Preventive Child Health Services in the Netherlands have already been doing this. Although the scope of the dissertation was drawn as 'major adult onset chronic diseases', it is worth to take a brief look at this example in the wider context of this general discussion. Preventive Child Health Services offered by well-baby clinics (Consultatiebureaus) for the ages of 0-4 and regional public health centers (Gemeentelijke Gezondheidsdiensten, GGD) for 4-18 include both individual and collective services [38]. In the individual level, children are invited for standardized health examinations at specific intervals: 15 times between 0 and 4 years of age and 3 times in the ages of 4-18. They are screened for a number of physical, social and psychological problems, the growth is monitored, and parents are provided advice based on specific needs, which are all interventions in line with the 'health oriented' paradigm. Nevertheless, several of the Preventive Child Health services are still 'disease oriented'. The approach to psychosocial problems of children is an example to this. If the symptoms of the child lead to a diagnosis, further investigation and therapy is offered. However, if the symptoms don't cluster into certain diagnostic criteria at that moment, the child and the family might not get the help they need. This 'disease oriented' paradigm can create a hurdle for early interventions to neurodevelopmental problems.

The underlying routine screening structure with standardized measures provides a valuable opportunity for the Dutch Preventive Child Health Services to monitor the child in a prospective way and predict the child's physical and psychosocial development, as well as any potential problems in those areas. In addition, effective integration of different assessment components can enable looking at the child in a holistic way. Thus, Dutch Preventive Child Health Services hold a huge potential to transform into a more predictive, preventive, participatory and 'personalized health care' model [39]. Personalized health care practices such as Gentest might inspire such transformations in the whole spectrum of health care, including child health services. 
The idea of targeting the whole population for primary prevention with individual level interventions seems to be also gaining its place in the literature [40, 41]. As they argue for the case of stroke prevention, by using ICT, it is easier to reach masses while individualizing the intervention.

Considering the promising role personalized health care models such as Gentest can play in reviving non-vertical comprehensive HFA strategies and considering the co-productive nature of innovation and health system as discussed above, one can conclude that, if we want to introduce practice models such as Gentest, we will need a health system that is ready to host comprehensive, prevention based approaches to health, risk and disease. So, when considering the introduction of personalized health care into the health system of a particular country in Europe we need to also assess its readiness for such an approach.

\section{Primary, secondary and tertiary prevention}

Classically, primary prevention is the effort to prevent a healthy person from falling ill. Secondary prevention entails the efforts to early detect a disease, so that the progression can be arrested and if possible the disease can be eradicated. Primary prevention is practiced prior to the biological origin of the disease, where secondary is practiced after the disease can be recognized, but before it has caused symptoms for the individual [42]. Tertiary prevention is aimed at arresting the progress of established disease. The target group for primary prevention includes those that are healthy, with respect to a target disease. Secondary prevention targets people who are already ill without being aware of it. Tertiary prevention is directed towards people who are already known to have a disease. Therefore, actually it is a form of care.[43]

Approaching prevention at primary and secondary levels started with the Commission on Chronic Illness in 1957 and later, the tertiary prevention is added to them. In 1983, Gordon published a critique and an alternative classification. In his critique, he reminded that the classification of primary, secondary and tertiary prevention arose in an era when concepts of health and disease were principally mechanistic [42]. In this way, this classification seems to be in line with the reductionist approach dominant in that era, which assumed 'single cause -> single disease -> single treatment', which was briefly introduced in Chapter 1. Primary, secondary and tertiary classification is mostly useful in responding to acute diseases because one can specifically tell the time of their onset. However, in chronic complex diseases, the disease progression is gradual; one cannot tell the exact time of the onset of, for example, diabetes of a person. This creates a problem in differentiating 'primary' and 'secondary' prevention. Moreover, for example, the end-organ damage already starts even before the onset of the diabetes; higher than normal blood glucose (not diabetic yet) initiates the end-organ damage. So, there is a continuum in development of chronic diseases that is dispersed in decades, which cannot be captured by the classical reductionist approach. 
With practical considerations that govern proper application of preventive interventions by health authorities, Gordon proposed an alternative 'operational classification of disease prevention'. Here, 'Universal prevention' is a measure that is for the general population regardless of the risk profile, 'selective prevention' is for sub-groups who have increased risk, and indicated prevention is for individuals who have found to be at sufficiently high risk to require preventive intervention [42]. As Gordon's classification is also about whom to approach individually and whom to approach collectively, based on the arguments conveyed earlier, it is not applicable to the case of a personalized health care model that aims to provide individual level prevention to the population at large. We need an approach that indicates the point and nature of intervention in the continuum of disease development, as depicted in Figure 2-a.

If we look at our case of Gentest from the classical primary, secondary, tertiary prevention approach, we see that it has the potential to cover the whole prevention spectrum. The purpose of this practice model is prevention of the major complex diseases and their complications, within the whole continuum (from the point where there is no disease to the point where there is fully established disease). Based on the case it is applied to, it can serve as a primary, secondary or tertiary prevention tool.

When implemented on a healthy individual, the practice model serves as a primary prevention tool since it aims to prevent development of the disease. If there are no risk factors, the individual receives nutrition and exercise recommendations based on his/her health priorities with the practice model.

If the disease hasn't fully developed yet, but there is a progression to the disease and individual has properties that put him/her into a 'risk group'; for example for diabetes such as the weight, waist circumference, age, some laboratory tests such as plasma glucose and LDL-cholesterol; the practice model serves as a secondary prevention tool. It can also be considered as a 'risk screening' tool.

If the disease has developed, then the practice model will serve to prevention of the complications of the disease, such as prevention of cardiovascular and kidney diseases for diabetics.

The extent of the benefits gained by the practice model is expected to diminish from primary towards tertiary prevention. Thus, it would likely be most beneficial for people without disease, and help prevention of and/or delaying development of diseases. It would probably be less effective for individuals with risk factors, compared to people without any disease, but it is still very beneficial to manage their risks. The recommendations may help stopping the progression of or delaying the onset of the disease, such as diabetes or heart attack. For people with developed disease, the benefits would be less, compared to people without the disease but it is still a valuable tool to support the management of the 
disease. With this practice model, for example, the primary care physician can see the risk of chronic kidney disease of a diabetic. For a cancer patient, the practice model will advise on diet during and after treatment. For a person with ischemic heart disease, the omega-3 fatty acid need will be calculated. So, at whichever point the progression of the disease is caught by the practice model, it is beneficial, but, if the person would have got it done in earlier phases, it would have probably been more beneficial.

It should also be noted that since the practice model Gentest has multiple diseases in its scope, it can be a primary prevention tool for disease A, while it is a secondary or tertiary prevention tool for disease B. For instance, for a patient with cardiovascular disease, it will serve as a tertiary prevention tool for cardiovascular disease whereas the nutritional advice will help for the primary prevention of cancer. Overall, it aims to prevent the onset and/or progression of the risk factors and diseases, in the whole continuum.

Thus, this practice model serves to 'personal health management' of individuals with no health problem, 'risk management' of individuals with risk factors, and 'disease management' of individuals with a manifested disease. As further tools that approach individuals in a comprehensive way for prevention of complex diseases emerge, the 'primary, secondary and tertiary prevention' terminology can be challenged and perhaps gradually replaced by the 'personal health management', 'risk management' and 'disease management' terminology, which suits better to the case of prevention of chronic complex diseases. The timing of these preventive approaches in relation to the 'disease development curve' is visualized in Figure 2-b.

Until now, I proposed to revisit two concepts that can be regarded as 'classical' in prevention. First one is that population based and high-risk individual prevention strategies are not the only options for prevention because with the introduction of tools such as Gentest, individual level interventions can be provided to large population groups. Secondly, I proposed that considering the continuum of development of chronic diseases, 'personal health management', 'personal risk management' and 'personal disease management' can be an alternative to primary, secondary and tertiary prevention classification. Although they are not traditional, they are still compatible with HFA, since these are different ways of ensuring to maintain and promote health, instead of providing solely disease oriented approaches.

After reviewing of both concepts, it is seen that an ecological approach to health is required to address all relevant determinants of health with 'tailored' and/or 'personalized' practices. Health systems need to innovate to accommodate to this ecological approach. Instead of focusing on "one problem at one visit" in a reactive manner, the services should approach the individuals proactively and provide 'tailored' health promotion and prevention interventions under integrated health management, risk management and disease management programs. Such personalized health care practices should cover the health 
of the individual in a holistic way, rather than focusing on specific lifestyle or disease area, or starting at a certain level of progression towards the disease (such as practices that target only people with risk factors or only people with certain diseases). The use of technologies such as ICT will very soon allow us to provide such services to the whole population. But, to enable that, health systems must move away from the dichotomy of 'population based vs. high risk individual' interventions and embrace proactive personalized health care approaches that can target the whole population to manage each individual's health, risk factors and diseases.

\section{The concept of 'public health' and place of 'personal' and 'population based' health services within 'public health'}

Chapter 2 (Part I) stated that 'public health' is a contested term and has different connotations, mainly depending on the use of the word 'public'. Some use it to refer to 'public health services', meaning either as 'publicly provided health services' as opposed to privately provided ones (such as public health insurance vs. private health insurance), or 'services provided to public, i.e. population, as opposed to the ones that are provided at the personal level. In either way, using the term public health to cover a set of services would make its scope narrow and demean it. Instead, we proposed, public health term must be defined and used in its broadest sense, i.e. "the attainment by all peoples of the highest possible level of health", which is the objective of HFA, as well as the WHO as described in its Constitution. Thus, everything that relates to a society's health should be categorized as a part of public health. This allowed us to frame every effort for health under the broad scope of 'public health', including health system, as well as the personal and population based services under it. The overall conceptual framework was called 'Health Globe'.

Personalized health care practices, such as Gentest, will enable us to manage the health of all, or at least a large part of, the individual citizens of a particular society, thus reaching beyond the population versus individual controversy. Moreover, Health Globe considers all health interventions a part of 'public health'. It uses this term in the broadest sense: everything that relates to a society's health should be a part of public health, in the sense that they aim "the attainment by all peoples of the highest possible level of health". Thus, developments in personalized health care would fit the Health Globe framework without any problems. However, if public health is used to cover only a set of services, positioning of an individual level intervention that targets large population groups, such as Gentest, would be disappointing because it will not make the fullest use of such practices and will miss a good opportunity to progress on the road to the long delayed HFA. 


\subsubsection{Back to basics: primary care services for prevention of chronic dis- eases}

The earlier pages emphasized the importance of changing mindsets and adapting health systems to support potential solutions to reduce burden of chronic diseases. Nevertheless, in order to approach individuals within their ecological contexts in a comprehensive way and manage their health, risks and diseases effectively, there is one area where we need to go back to decades old recommendations: strengthening primary care services, while emphasizing the need for innovation there.

Primary care is the most suitable line of health services to prevent chronic complex diseases with a comprehensive, proactive and personalized approach [44]. Within the Health Globe framework (Chapter 2), health services were described in 9 levels of services. Personal health promotion, personal disease prevention, primary diagnostic and curative and primary care rehabilitative services were defined to be the services provided by 'primary care services' (Figure 4). Here, practice model Gentest can serve as a useful tool for personal health promotion and disease prevention services.

Primary care services in their current form are not strong enough to provide services for prevention of chronic diseases effectively. Their strengthening has been among the recommendations of various major health policy documents since 1980s. It is known that countries with stronger primary care services have less rates of all cause mortality and all cause premature mortality, in particular mortality cause by chronic diseases [45]. The importance of primary care services was also emphasized in Chapter 3 , in the context of the U.S. health system, which has a weak foundation for primary care, as compared to other high-income countries [46].

To strengthen primary care services, we need to innovate and develop new models of delivery. This need is emphasized by various recent reports around the globe [44, 47-49]. They address the need for more proactive, preventive and personalized services provided by multidisciplinary teams, as are the needs of the overall health system. They also ask to approach individuals considering their social and ecological contexts. Among those reports, a comprehensive view to primary care services was introduced by WHO in World Health Report 2008 under 'people centered primary care' [47]. The properties of this approach in comparison with conventional medical care and programs that are oriented towards controlling diseases are presented in Table 1. 


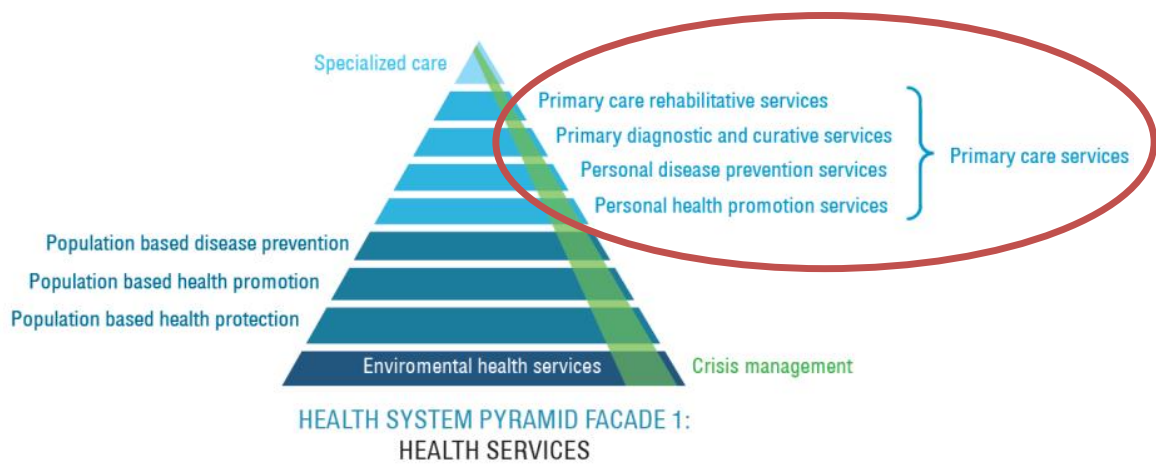

Figure 4: Health Services Facade (Facade 1) of the health system pyramid within the Health Globe framework

Primary care services are highlighted with a red circle to emphasize where Gentest will be integrated.

Table 1: Aspects of care that distinguish conventional health care from people-centered primary care

\begin{tabular}{|c|c|c|}
\hline $\begin{array}{l}\text { Conventional ambulatory } \\
\text { medical care in clinics or } \\
\text { outpatient departments }\end{array}$ & Disease control programmes & People-centred primary care \\
\hline Focus on illness and cure & Focus on priority diseases & Focus on health needs \\
\hline $\begin{array}{l}\text { Relationship limited to the moment of } \\
\text { consultation }\end{array}$ & $\begin{array}{l}\text { Relationship limited to programme } \\
\text { implementation }\end{array}$ & Enduring personal relationship \\
\hline Episodic curative care & $\begin{array}{l}\text { Programme-defined disease control } \\
\text { interventions }\end{array}$ & $\begin{array}{l}\text { Comprehensive, continuous and person- } \\
\text { centred care }\end{array}$ \\
\hline $\begin{array}{l}\text { Responsibility limited to effective } \\
\text { and safe advice to the patient at the } \\
\text { moment of consultation }\end{array}$ & $\begin{array}{l}\text { Responsibility for disease-control } \\
\text { targets among the target population }\end{array}$ & $\begin{array}{l}\text { Responsibility for the health of all in } \\
\text { the community along the life cycle; } \\
\text { responsibility for tackling determinants } \\
\text { of ill-health }\end{array}$ \\
\hline $\begin{array}{l}\text { Users are consumers of the care they } \\
\text { purchase }\end{array}$ & $\begin{array}{l}\text { Population groups are targets of } \\
\text { disease-control interventions }\end{array}$ & $\begin{array}{l}\text { People are partners in managing their } \\
\text { own health and that of their community }\end{array}$ \\
\hline
\end{tabular}

Source: World Health Report 2008: "Now more than ever" [47].

Our practice model Gentest can potentially be a tool that supports and empowers 'people-centered primary care' in many of the aspects listed in Table 1. These will be introduced briefly below. 
Focus on health needs: The mission of our practice model is to change the behavior of the individual and allow the primary care physician (general practitioner/ family physician, abbreviated as GP) to manage the health of the individual according to his/her priorities. This allows the primary care team and the individual to tailor the lifestyle and medical follow-up plans according to the individual's health needs.

Enduring personal relationships: At a first glance, it might look counterintuitive that the practice model may improve 'relationships', since the Gentest report will be produced by software and will be communicated, partly, via computers. However, if used effectively by the primary care providers, it actually may provide a communication ground on the personal conditions and various lifestyle habits of the individuals.

Comprehensive, continuous and person-centered, as opposed to program-defined 'disease control' interventions: Programs that focus on prevention of chronic diseases have been mostly following a model orientated towards a single specific health issue. Each major disease or risk condition has been seen as the starting point for the development and implementation of a specific prevention program, separate from other diseases or conditions and therefore known as a vertical program ${ }^{3}$. These vertical programs are thus not integrated and this is reflected in the multiplicity of chronic disease prevention initiatives that do not necessarily relate to one another. However, integrated approaches are needed to maximize the benefits of investing in prevention and lead to more effective results. [50]

Our practice model may serve as a powerful approach to integrate the prevention efforts in primary care services, with a focus on the diseases and risk conditions that cause the highest burden in the society. Instead of running separate programs such as cardiometabolic risk assessment and management, healthy nutrition (for example increasing fruit and vegetable consumption), smoking cessation, and physical activity and exercise, a single tool can be used to help manage main lifestyle factors and disease risks. If integrated as envisaged in the ideal case, i.e. by becoming an integral part of the health records of the individual and with the follow-up program, it can become a tool for the GP to manage the health of people that are registered to his/her practice in a continuum.

People are partners in managing their own health: The participation of the individuals in the follow-up program with online tools is likely to empower and engage individuals to take control of their health.

\footnotetext{
${ }^{3}$ Vertical programs reflect the 'combating disease paradigm', which was the focus of public health interventions in the last century, in particular the period before the HFA approach was introduced (see sub-section 1.1 in this chapter and Chapter 2).
} 
As seen above, the practice model can be a useful tool for the primary care services to provide the 'people-centered' care, as envisaged in the World Health Report 2008. On the other hand, accommodating to host personalized health care practices such as our model, might help primary care services evolve towards a 'people-centered care', signifying the 'co-evolution' of the system and the practice (Figure 1).

It is of note that in the recent years, publications have started emerging in the literature for reporting implementation of cardiovascular risk assessment and management programs in primary care services as personalized preventive interventions [51-54]. This also shows that there is need for timely and valid introduction of Gentest into primary care services.

At this point, it is important to note a perception of primary care to be often seen synonymous with simple and low-tech services provided by not so skilled professionals, particularly in case of low-resourced settings/ low income countries. This is a dangerous oversimplification, as the World Health Report 2008 warns [47]. As the primary care services are strengthened, their resources, i.e. infrastructure, human resources and knowledge, also need to be improved and developed. In terms of the costs, what matters should not simply be the cost of the intervention, but the health gains achieved with the costs, i.e. costeffectiveness. ICT can provide important tools that help to improve efficiency and effectiveness in primary care. As the cost of genomic technologies is rapidly decreasing, their usage may become cost effective in population wide strategies.

\subsubsection{Properties of health systems that would better facilitate the integra- tion of a preventive personalized health care model}

Chapter 7 proposed a systematic approach to assess how the practice model Gentest can be integrated into a health system. For this purpose, 24 areas of assessment, as well as numerous issues and criteria under them, were identified. Additionally, examples from the Netherlands and the UK, as well as other relevant European settings, were provided to illustrate these areas. This work also included several directions important to answering the question 'What kind of health systems would better facilitate the integration of this practice model?'. The answer to the question that was already covered in Chapter 7 will be summarized here in a more explicit way.

- Progress in the paradigm shift

As highlighted in Chapter 1, as well as the first pages of this chapter, health systems need a paradigm shift from reactive, disease oriented, acute diagnostic and curative services focusing on medical services towards proactive, preventive, health oriented, focused on chronic cure and care covering the whole spectrum of health care. This also calls for an ecological approach to health, which aims to understand and tailor the services according to the context of the individuals. Our practice model provides an example to this new 
paradigm. It approaches individuals in a comprehensive manner and aims to cover a wide range of determinants of health. It aims to prevent the development and progression of chronic diseases in a proactive way.

For successful integration, the health system that will host the practice model should already have started its transformation towards the new paradigm, or at least have strong willingness to do so.

- A strong primary care system and its scope in terms of (primary) prevention

A strong primary care system with a good focus on prevention is a prerequisite for its effective integration. If the GP is not a 'gate keeper' in the health system, its effectiveness is very likely to be diminished. If the GPs don't have the mandate to manage the health of the individuals, don't have prevention among its tasks, and focus only diagnosis and treatment (reactive approach), their willingness to implement it in their practice would probably be low. Countries with weak primary care services wouldn't be a suitable place for implementation of the practice model, unless it is considered to be embedded within a program on restructuring and strengthening of primary care services.

Thus, a strong primary care is a prerequisite, but its not enough. Primary care should also have a mandate towards preventing chronic diseases, and not focus only on medical interventions, i.e. but also be ready to embrace to providing lifestyle interventions.

- $\quad$ Financing system towards awarding outcomes

The financing system should be more towards awarding health outcomes, rather than processes or outputs, so that the GPs can be incentivized with the possible health gains due to the practice model.

- $\quad$ The positive environment towards change

Integration of the practice model will require changing of conventional mindsets in the health system. As raised in earlier sections of this chapter, classically, interventions for primary prevention of chronic diseases have been assumed to target the population level. As covered earlier, the practice model challenges this approach by providing individual level services for primary prevention, while still targeting the whole population. Thus, to understand the model and make an informed decision on its integration, the stakeholders and health authorities will first need to 'think out of the box'.

Moreover, reorganization and redesign of preventive services in primary care will highly likely be required for successful implementation of the model, signifying the importance of 'co-evolution' conveyed in Figure 1. It also requires change management and, to some extent, social engineering focusing on health sector. Thus, the attitude and openness of 
the health sector as a whole towards innovations is an important factor in the integration of the practice model.

In some cases, there might be willingness to attain the paradigm shift and integrate the practice model to the health system, but the system might not be in the desired status. In these cases, the practice model can also be used as one of the vehicles to carry out the desired transformation. For example, if the country doesn't have a strong primary care system, but there is a willingness to transform towards that, our practice model can be embedded within a program on restructuring and strengthening of primary care services. Such cases would create concrete examples of the co-evolution of the health system with a personalized health care practice (Figure 1).

Overall, the example of our practice model indicates that developments in the field of personalized medicine and health care will have a profound effect on how services are delivered, and could eventually lead to service redesign and reorganization. Such applications may actually be used as a practical opportunity to realize the paradigm shift required in the health services, i.e. towards services that are proactive, health oriented, focused on chronic cure and care covering the whole spectrum of health care. This means, the health system and the personalized health care innovations need to evolve together, leveraging one another (Figure 1) towards the goal of 'attainment by all peoples of the highest possible level of health', i.e. HFA.

\subsection{Innovations for personalized health care in light of health sys- tems}

In sub-section 1.2, the co-evolvement concept was introduced: health systems must innovate to better host 'personalized' practices and 'personalized' practices must innovate to better fit into the health system context (Figure 1). In line with it, the previous section looked at the potential areas of innovation for the health system. Now, it is turn of personalized medicine and health care to feed into co-evolvement introduced in Figure 1. The first heading of this sub-section will briefly discuss how 'personalized' practices should innovate to serve to the goal of HFA. Then, the room for innovation for a specific personalized health care model (Gentest) will be presented.

Chapter 4 provided examples of personalized practices from USA, France and Taiwan to show how health system context matters when it comes to their integration into health services. Chapter 7 took it further and provided a systematic approach to identification and assessment of integration issues. The last heading in this sub-section will provide additional argumentations to emphasize why the field of personalized medicine and health care needs to be aware of the importance of the health system context to reach successful integration of the tools they are developing, into health systems. 


\subsubsection{The need for innovation for 'personalized' practices}

Just like health systems, personalized practices also need to innovate towards proactive, preventive, health oriented, focused on chronic cure and care covering the whole spectrum of health care, to serve to the goal of HFA. But, how can they do that?

To understand which direction 'personalized' practices should innovate towards, we need to understand what 'personalized' practices are. Chapter 4 presented a study that identified 88 'personalized practices' in the scientific literature with a systematic search and inclusion strategy, and analyzed their content. The results showed that 'personalized' practices by no means are a homogeneous group that can be described in a standard way. They contain different practices (commodities in health care market or implementation models on how health services are provided), with various purposes (prevention, early detection, diagnosis, treatment, prediction of prognosis, etc.) and serving different groups (individuals/patients, health professionals, health care organizations, etc.). To 'personalize' medicine and/or health care, some of them focus mainly on technologic aspects, such as genomic technologies or ICT, while some of them only use behavioral aspects. In particular, many of the ones that used genomic technologies to 'personalize' the services focused on a test (which may include single or multiple markers) that leads to a specific decision in medical processes such as using a drug or not, without taking into account other determinants of health. This reflects a 'single test -> single intervention' understanding. Chapter 1, pointed that an earlier reductionist approach in medicine and health care assumed 'single cause -> single diseases -> single cure' [55], and emphasized that health systems must transform towards a more proactive one that takes a holistic and integrative approach towards health and disease. The 'single test -> single intervention' couples, in a way, also exhibit a similar understanding in the sense that they try to reduce the complexity of health and disease into a single test and single intervention. Is that the 'personalization' we'd like to achieve? Is every 'test' that uses the genome based information of a person means 'personalizing' medicine and health care?

As was conveyed in Chapter 4, excessive technological focus, in particular for genomebased technologies, might actually be hampering the progress towards 'personalization' vision for two reasons. First of all, it creates attribution of currently unfulfilled expectations of genomic medicine to the broader application of 'personalization' vision. Secondly, as also raised in Chapter 5, genomics is one of the components, but it is not the only factor that makes individuals unique or medicine/ health care personalized.

However, focusing on only 'non-biological', or behavioral factors to 'personalize' health and health care would also not be a truly holistic approach, since, as suggested in Figure 3, all levels including biology, psychology and social and ecological context interact.

Within the 'personalization' vision, it is very important to realize that 'personalization' can only be achieved when we understand and use the individual contexts in a holistic way, 
rather than focusing on a single factor, be it high-tech or low-tech, biological or nonbiological. Each person is unique within their own context that is constituted by multiple (levels of) determinants of health, as presented in Figure 3. To truly personalize health and health care, we need to develop comprehensive approaches that take this unique context into consideration. This means, personalized health care practices should ideally consider all available/possible determinants of health that can be used for a specific purpose, as shown in Figure 3. By careful analysis of each case, it can be possible to identify which interventions might be most effective for the individual context. Moreover, instead of thinking merely in singular interventions, multiple ones should be provided, as much as possible, in an integrative manner towards the desired effect. All these call for being comprehensive in terms of the integrated bio-psycho-socio-ecological approach to health, while personalizing and integrating the health care.

If we look at the 88 practices identified for the study in Chapter 4 , it is seen that only a limited number of them involved multiple determinants of health, and among them, only a handful had some sort of holistic approach towards health, which considered the unique context of individuals that are constituted by multiple (levels of) determinants, at least to a certain extent, and claimed to provide multiple interventions for them [56-62]. This shows that, just like health systems, personalized medicine and health care innovations also need to work hard to evolve towards a holistic, comprehensive and integrative approach to personalize health care.

\subsubsection{Innovation to adapt to target countries and health systems}

'Personalized' practices need to continue to innovate in order to adapt to the needs and conditions of the health system. Thus, as was introduced in Chapter 4, innovation does not follow a simplified linear process or a 'pipeline', which ends when the 'product' is out in the market. 'Personalized' practices and the health systems continue to co-evolve (Figure $1)$.

Gentest was developed and is currently provided in Turkey as a service to individuals who are willing to get it and are eager and able to pay for it. The way it is implemented in Turkey has been briefly described in Chapter 6 . But, how can that be a part of primary care services? How would it be implemented there? To help us imagine that, a brief vignette is presented in Box 1 on a fictitious individual and a primary care setting.

\section{Box 1: Vignette for the practice model implemented in a primary care setting}

Mr. Yilmaz, who is a 50-year-old man, receives a letter from his GP to participate a new health check, with some leaflets. He reads that this check will evaluate him 360 degrees with his personal health information, lifestyle factors (nutrition, exercise and smoking), body composition, genetic structure and other biomarkers (cholesterol, glucose, etc.). He is asked to devote a good two hours' time for the first appointment, as it involves filling in 
a detailed online questionnaire with team of his GP. He should also drop by another time after an overnight fasting to give blood and urine sample. He makes an appointment to do both on Monday morning. For more information, there is a brochure he is encouraged to read until the appointment.

On M onday morning when he goes to the GP, he is taken to a room where he watches a video of about 10 minutes that explains all the procedure, what the results will tell and how they will look like. This is in line with the information in the brochures. Then, the nurse practitioner comes in to ask him if he has any questions. After answering his, it is the nurse's turn to ask him questions to validate that he understands the procedure. Then, he reads and signs a consent form.

The nurse draws blood (for conventional biomarkers and genetic testing) and then makes several measurements, including weight, height, waist circumference, fat ratio (bioimpedance measurement), pulse and blood pressure. Afterwards, he sits in front of a big screen computer to fill in a detailed questionnaire including these areas:

- Personal data

- Medical history

- Family history

- $\quad$ Living and working conditions

- $\quad$ Physical activity and exercise

- $\quad$ Smoking and drinking habits

- Supplement consumption

- Nutritional habits

- Food consumption

While filling in the food consumption data, he answers the questions using the photographs on the portion sizes of different food items. He is also offered a choice to make a record of all he eats for three days using an app for his smartphone. At the end of the appointment, he is curious what will come as a report.

The information retrieved today will be an integral part of Mr. Yilmaz's patient profile and thus, his GP will use the inputs, as well as the report he will get, to manage his health and any upcoming disease.

The information collected, including the measurements and questionnaires, goes to a central server. The blood sample is analyzed in a local laboratory for conventional biomarkers, whereas an additional tube goes to a central lab for genotyping. Results of these tests also go to the central server. 

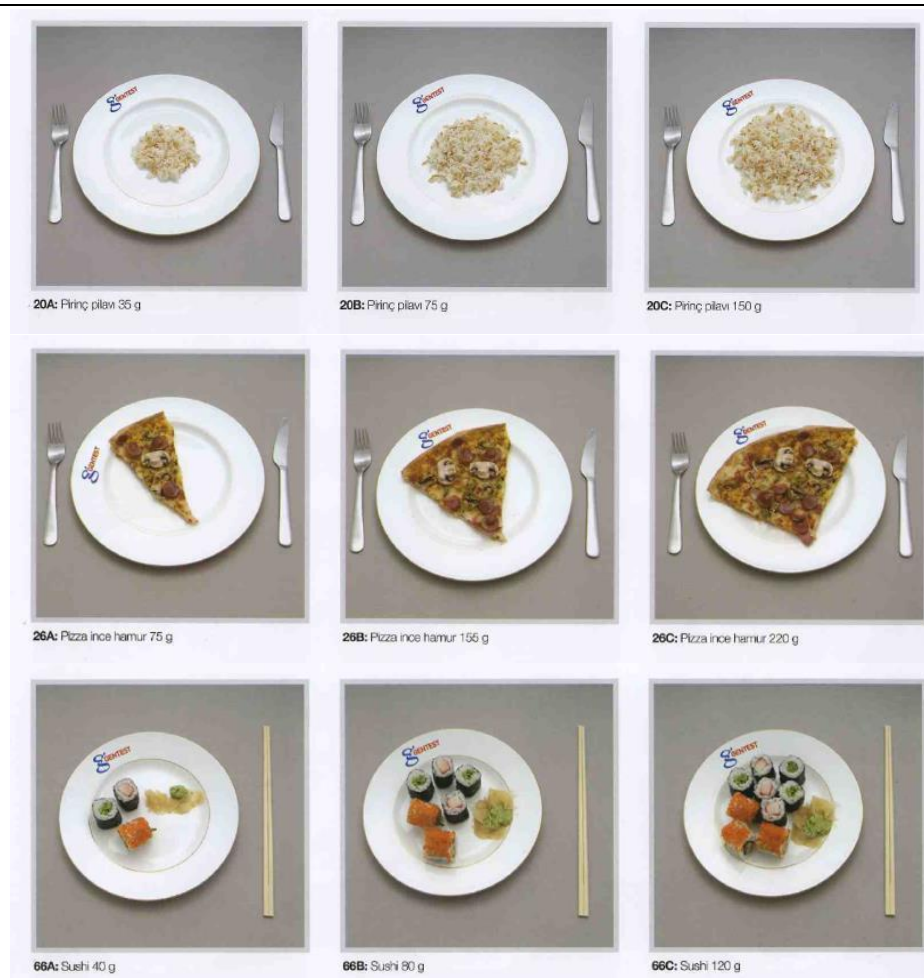

20B: Prinç pliaw 75

20C: Prinç plav 150
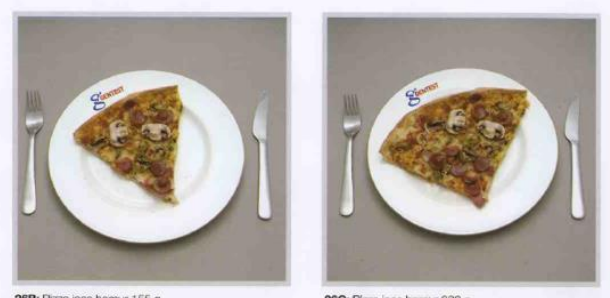

268: Pliza inoe hamur $156 \mathrm{~g}$

26C: Piza ince hermo 220
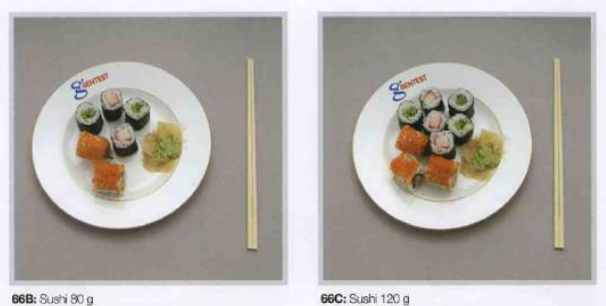

Figure 5: Images from the food portion size atlas

All the data is processed to produce a 'health check' report in the central server. Here, his current status in terms of body composition, nutrition, exercise, smoking (including causes of smoking), health information (including blood pressure, pulse, and laboratory test results), and genetic markers are summarized. Then, risk assessments for most common chronic complex diseases are presented. These include myocardial infarction, stroke, type 2 diabetes, osteoporosis and most prevalent cancers (lung, breast, prostate, colon and stomach). This includes the current status of his risk profile, as well as potential decreases if he adopts the optimum lifestyle and medical follow-up plan. The report of an individual includes the results of the analysis and an optimum lifestyle plan developed for this individual. It includes a plan for reaching and/or maintaining optimum body composition, menu plans and exchange lists, a supplement plan, and an exercise plan. Personalized smoking cessation recommendations are given based on the individual causes of smoking. Recommendations are given for medical follow-up with personalized screening plans. Each report can be checked and modified by the GP, if she deems necessary.

In the 4 weeks period when his report is prepared by the central server, Mr. Yilmaz goes to a seminar organized by his GP practice. They are offered once every week to all people who are participating the 'health check'. The seminar covers various topics needed to 
understand the results of the report, such as how genetic structure interacts with lifestyle factors, what 'risk' means for different diseases, how they are calculated, why healthy lifestyle is important for healthy and long life, etc.

When his report is ready, Mr. Yilmaz is invited to visit his GP for an hour. At the appointment, he first shortly visits his GP, who presents his printed report to him. She provides an overview of the results to Mr. Yilmaz and any issues that have medical significance. With his report, he then moves to another room to meet with the nurse practitioner to talk about optimum lifestyle and medical follow-up plans in more details. The nurse makes some modifications in the lifestyle plan according to his wishes and possibilities.

Since he doesn't have serious risk factors (such as obesity or other risk factors that require a more intensive lifestyle program), his follow-up visit with the nurse practitioner is planned for 6 months later. Meanwhile, he can record his food consumption and exercise online to see if he is reaching his goals. He can use a smartphone app for these too. Nevertheless, he can call or take an appointment from the nurse when he has questions, or contact her online, which might be more practical.

This vignette presents very briefly how one can imagine Gentest taking place in a primary care setting. While reading it, one would start asking several questions on how things should be arranged, such as the data collection and storage, ethical issues, the role of different personnel working in a GP premise, how they will be trained, the additional workload that will come with such an application, the required infrastructure, how the GP will be paid for this, if the GP (and the individuals) would like to implement it, etc. The answer to them, and several more, would depend on where it will be used/ practiced. That's why, a systematic approach was proposed in Chapter 7 to identify and assess these issues and others.

In the example of our practice model, the health system context will probably need to innovate to be able to host it and yield most effective results. On the other hand, the practice model also needs to innovate to adapt to the specific setting. Simply translating the questionnaire and reports will definitely not be enough. For example, the tools used to measure lifestyle need to be adapted for each target country. For each country or group of countries, new nutritional questionnaires and food portion size atlases must be developed, or adapted from already existing ones. As it needs to be optimized at primary care settings, the current questionnaire will probably needed shortening, as well as the report. For standard information that the individual needs to use to understand their reports, educational videos can be prepared to save the time of the staff at the primary care setting. Seminars can also be carried out in certain days of the month to convey such standard information. Further tailoring of reports for different health literacy levels might be considered. Most importantly, the knowhow behind the practice model needs to be trans- 
lated into a fully operational software to enable its 'mass production' in large population groups.

As any other intervention, our practice model also needs to be revised and accommodated to use the latest developments in the scientific and technological fields involved. The advantage of the practice model is that its components can be updated while maintaining the integrative approach behind it. The main innovation lies in the comprehensive approach that takes the health and behavior of the individual as a whole. So, instead of having separate programs for healthy diet, exercise, smoking cessation, etc. in terms of lifestyle, and cardiovascular risk management, diabetes risk management, cancer risk management in terms of diseases; this practice model can be implemented as a single program to manage the health of individuals in primary care services.

The update would involve contemporary approaches in all relevant fields, including genomics, lifestyle assessment methods, lifestyle interventions, in particular the ones developed with 'tailored' approach in health promotion and education, disease risk assessment, screening and follow-up guidelines, and many others.

It is of note that effectiveness and cost-effectiveness of the practice model hasn't been demonstrated yet. Chapter 6 stated that designing and conducting a study that would collect data on the effectiveness of the model was envisaged after its pilot. However, due to external conditions and constraints of the funding environment in Turkey, it was not possible to carry it out. For the case of integration of Gentest in Europe, after the adaption and update, as summarized above, the next step needs to be a pilot and, then, evaluation study in a country where there is willingness to integrate this practice model. Medical Research Council of UK provides valuable guides for evaluation of complex interventions, such as our practice model [63-65]. Since this practice model is not only a complex intervention but also one that require service re-design and re-organization while it is integrated, the pilot and evaluation sites will need to go through important changes in their organization and service delivery. As we've briefly listed above, the practice model also needs to evolve, to accommodate to the specific case of the health system, leading to their coevolution (Figure 1). Thus, the results of the evaluation would depend on not only the intrinsic qualities of the practice model, but also how well the context is adopted for its integration. Therefore, the pilot and evaluation process would probably not only evaluate the practice model, but also the whole co-evolved program with newly designed services and organization to a large extent.

A strategy to facilitate decision-making could be to pilot the program in which the practice model is implemented in a limited geographical area, and then, to gradually expand towards a larger area as the collected data indicates favorable results. The short-term evaluation could be made on surrogate markers and as decisions are made for gradual expansion, long-term outcome data would become available. Since it will not be possible for 
decision makers to have conclusive decisions while on surrogate markers, an option for financing would be 'access with evidence development', or in other words, conditional coverage [66]. This is a reimbursement policy option that go beyond the traditional 'yes', 'no', or 'yes with restrictions' options. 'Access with evidence development' models serve to promoting research and development [67], while reducing the risk for the health system payers.

In the further future, data generated with this population wide application in primary care services can create a feedback loop to research with large amounts of data ('big data'), providing valuable insights to health and diseases. The mining of this data can help to better define health and disease, discover who is more likely to respond to which lifestyle interventions and develop more accurate risk assessment tools.

\subsection{3. 'Personalized' practices in health system contexts}

Chapter 7 reported that the documents, reports and discussions on integration of 'personalized' practices into health services mostly focus on diagnostics and pharmaceuticals and provide a bullet list of 'barriers' or 'challenges' that need to be overcome to deliver personalized medicine and health care to the society. An important point here is to realize that the problems of the health systems are actually reflected in those reports and discussions in a profound way. What would happen if we remove the words 'personalized medicine' from the titles of the lists of 'barriers' or 'challenges'? It is highly likely that those 'barriers' will hold valid for any innovative practice introduced in those health systems, because those 'barriers' are probably the weaknesses of the system in its wider context, not specific to the 'personalized' practices. However, the field of personalized medicine and health care, which mostly consist of people from science and technology domains, spend considerable effort to find solutions to them while preparing those documents, as well as at various meetings, conferences, etc. At this point, it might be wise to turn to the field of health systems and policies to understand the underlying reasons and develop solutions to those problems, as was done in this dissertation.

Chapter 4 gave the example of USA, France and Taiwan as very different cases of implementing 'personalized' practices in countries. Chapter 3 summarized the problems of the U.S. health system in comparison to other high-income countries, independent from the purpose of integrating 'personalized' practices: low effectiveness, low efficiency and serious problems with inequity [46, 68-70]. As can be seen in Chapter 4, these problems have a profound effect on how 'personalized' practices are implemented in the USA.

Leroy Hood, an important opinion leader who has been pushing the field of personalized medicine and health care forward for more than a decade proposed that P4 medicine (a form of personalized medicine/ health care) would eventually lead to a universal democratization of health care by increasing the effectiveness of preventive and curative interventions, and reducing the costs, thus lessening the social and financial burden of diseases 
$[71,72]$. Indeed, as conveyed earlier in Figure 1, personalized health care can be used as a tool to innovate health systems. However, while envisaging that, there are two important issues that need to be taken into account.

First of all, in line with the 'co-evolution' concept presented earlier (Figure 1), personalized practices can help to transform health care and, thus, the health system, but alone, they cannot change the inherent weaknesses of a health system. Those weaknesses must be recognized and targeted with structural reforms, such as the ones recommended for the USA in Chapter 3. The other way of looking at the issue is probably also true. A strong health system, i.e. an effective, efficient and equitable one, will probably integrate innovations more easily and effectively. For example, in the example of France in Chapter 4, we see that France, whose health system satisfies these conditions to a large extent, has achieved implementation of a country-wide program for cancer genomics.

Secondly, if 'personalized' practices are to be used for transformation of a health system, a systematic approach towards health system must be taken. The one developed and presented in Chapter 7 is an example to that because it can also be used to map where our practice model can be used to facilitate the paradigm shift in the health system, as well as to pinpoint the underlying problems which probably cannot be overcome while integrating the practice model.

All in all, the field of health systems and policies has much to offer to the field of personalized medicine and health care to have a comprehensive approach to integration of 'personalized' practices. As raised in Chapters 4 and 7, effective communication, collaboration and interaction among different fields are crucially required to reach a more personalized, predictive, preventive and participatory health care.

\section{Methodological considerations}

Present research took place in a changing environment of the personalized medicine and health care field and was fed by the new emerging dimensions and visions. In addition, there have been no specific examples on analysis of integration of preventive 'personalized' practices in health systems. Therefore, rather than having a fixed PhD roadmap, this research evolved gradually and each stage of the research brought new questions and dimensions to address in the next stage(s) in order to meet the dissertation aim effectively. Some chapters evolved together, such as Chapter 7 and this chapter of General Discussion. Therefore, many of the concluding remarks were already highlighted in Chapter 7 against the recent literature. In this section, I will reflect on my PhD journey retrospectively. 
This journey started with the practice model Gentest, which I used as my vehicle. It helped to keep the research focused on a real life example, rather than floating on hypothetical cases or generic uses of genome based knowledge and technologies.

In this sense, the research approach used in this PhD is broadly in line with constructive research approach that is mainly seen in business administration and accounting, as well as engineering fields. Constructive research aims "...producing innovative constructions, intended to solve problems faced in the real world and, by that means, to make a contribution to the theory of the discipline in which it is applied." [73] It can be regarded as a specific approach under 'case study method' in social sciences. The critical point here is to focus on a real world problem and by doing that developing theoretically grounded solutions for practical purposes.

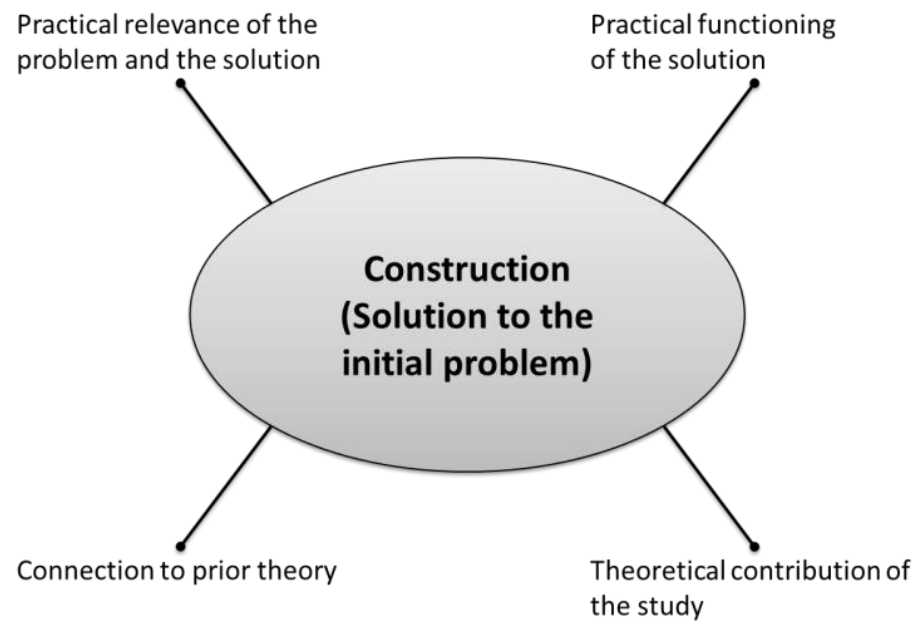

Figure 6: The central elements of the constructive research approach

Source: 'What is a constructive research approach?' by Lukka [73].

The PhD research didn't follow the specific process of constructive research, which had been described in particular for business administration and accounting field in a step-bystep manner [73]. Nevertheless, looking at my research retrospectively, I see that the approach used in this dissertation is generally in line with several central elements of constructive research (Figure 6). The starting point of this research was a real life problem (integrating Gentest to health systems) that is relevant to be solved in practice (the case is introduced in its larger context in Chapters 4-6) and produced an innovative construction to solve the initial world problem (as proposed in Chapter 7), which is linked to prior theoretical knowledge (Chapters 2 and 3 ) and has the potential to contribute to the theory in this field (Chapter 7). 
Use of a real life case example has been a very useful approach in this $\mathrm{PhD}$ research. First of all, it would not be possible to address 'integration' issues of all potential health care practices that are linked to personalized medicine and health care with a single approach. As seen in Chapter 4, the practices presented under personalized medicine and health care are very heterogeneous. There is no unified definition for them, nor a classification can be made using conventional frameworks. It is not possible to reach a solution that applies to all these diverse practices in a uniform way. Therefore, this research had to take a concrete case example.

Secondly, starting the $\mathrm{PhD}$ research with a real life example and its problem, i.e. integration into health systems, enabled the research to be more concrete, down to earth and practice oriented, rather than being theoretical.

Thirdly, while trying to solve the integration 'problem' for our practice model, we've developed a systematic approach that might be useful for other 'personalized' practices, as well as the innovations in health care in the broader sense. Using health systems framework to approach integration issues in a systematic way is an innovative solution and has also the potential to contribute to the theory in this field.

\section{Main conclusions}

This dissertation took a journey involving health systems and policies, prevention of chronic diseases, and personalized medicine and health care. Main conclusions also span in these diverse fields.

- The 'Health for All' (HFA) vision, i.e. "the attainment by all peoples of the highest possible level of health", still hold valid and provide a coherent overarching framework to the overall goal of public health. Public health is what we do to reach this goal. Since a health system is the main actor that serves to this goal, but not the only one, it is a part of 'public health'.

- To reach HFA vision, health systems must shift from being reactive, disease oriented, focusing on acute diagnostic and curative services with medical processes towards proactive and health oriented services focusing on prevention as well as chronic cure and care, covering the whole spectrum of health care (not only medicine).

- Personalized health care offers important opportunities to combat the increasing burden of chronic complex diseases while serving to the required paradigm shift.

- Innovations for personalized medicine and health care, and health systems and policies need to co-evolve, adapting themselves to the needs of each other while leveraging one another. 
- $\quad$ For successful integration of personalized health care practices, issues related to integration needs to be approached systematically, not in a piecemeal way. Health system frameworks can provide the required analytical frameworks to identify and assess issues related to integration of those practices.

- 'Personalized' practices are not a homogeneous group. They contain different practices (commodities in health care market or implementation models on how health services are provided), with various purposes (prevention, early detection, diagnosis, treatment, prediction of prognosis, etc.) and serving different groups (individuals/patients, health professionals, health care organizations, etc.). Therefore, it is necessary to focus on a specific case when aiming to identify integration issues.

- $\quad$ For successful integration of personalized health care practices such as Gentest, we need to overcome some traditional boundaries in terms of our approach to prevention.

- $\quad$ As in the whole health system, primary care services need to innovate to attain the HFA vision, towards the required paradigm shift, and to better host personalized preventive practices.

- $\quad$ 'Personalization' means providing a comprehensive approach to the individual within his/her unique context.

- $\quad$ Personalized practices such as Gentest also need to innovate to adapt to the health system where it will be integrated.

- $\quad$ An effective dialog among the fields of personalized medicine and health care and health systems and policies may facilitate better and faster integration of 'personalized' practices into health systems.

\section{References}

1. Paving the Way for Personalized Medicine: FDA's Role in a New Era of Medical Product Development: FDA - U.S. Food and Drug Administration; 2013. Available from: http://www.fda.gov/downloads/ScienceResearch/SpecialTopics/PersonalizedM edicine/UCM 372421 .pdf.

2. Personalised medicine: Opportunities and challenges for European healthcare - Workshop report, 13th European Health Forum Gastein, 7 October 2010: European Commission; 2010. Available from: http://ec.europa.eu/research/health/pdf/13th-european-health-forum-workshopreport_en.pdf.

3. Global Strategy for Health for All by the Year 2000. Geneva: World Health Organization 1981.

4. Kickbusch I. The Contribution of the World Health Organization to a New Public Health and Health Promotion. Am J Public Health. 2003 10/30/accepted;93(3):383-8.

5. Whitehead $M$. The concepts and principles of equity and health (EUR/ICP/RPD 414, 7734r). Copenhagen: World Health Organization Regional Office for Europe; 1990.

6. Targets for health for all: Targets in support of the European regional strategy for health for all. Copenhagen: World Health Organization Regional Office for Europe; 1985. 
7. M cLeroy KR, Bibeau D, Steckler A, Glanz K. An Ecological Perspective on Health Promotion Programs. Health Education \& Behavior. 1988 December 1, 1988;15(4):351-77.

8. Kreuter MW, Skinner CS. Tailoring: what's in a name? Health Education Research. 2000 February 1, 2000;15(1):1-4.

9. Kickbusch I, Gleicher D. Governance for health in the 21st century Copenhagen: World Health Organization Regional Office for Europe; 2012. 128 p.

10. M CQueen DV, Wismar M, Lin V, Jones CM, Davies M. Intersectoral Governance for Health in All Policies. Copenhagen: World Health Organization on behalf of the European Observatory on Health Systems and Policies; 2012. 206 p.

11. Health 2020. A European policy framework and strategy for the 21st century. Copenhagen: World Health Organization Regional Office for Europe; 2013.

12. Chin WW, Hammermesh RG, Huckman RG, McNeil BJ, Newhouse JP. Forum on Healthcare Innovation: 5 Imperatives Addressing Healthcare's Innovation Challenge: Harvard Business School \& Harvard M edical School; 2012. Available from: http://www.hbs.edu/healthcare/Documents/Forumon-Healthcare-Innovation-5-Imperatives. pdf.

13. Swanson RC, Cattaneo A, Bradley E, Chunharas S, Atun R, Abbas KM, et al. Rethinking health systems strengthening: key systems thinking tools and strategies for transformational change. Health policy and planning. 2012 Oct;27 Suppl 4:iv54-61.

14. Russell E, Swanson RC, Atun R, Nishtar S, Chunharas S. Systems thinking for the post-2015 agenda. Lancet. 2014 Jun 21;383(9935):2124-5.

15. Jasanoff $\mathrm{S}$, editor. States of Knowledge: the Co-production of Science and Social Order. New York: Routledge; 2004.

16. Snyderman R, Yoediono Z. Prospective care: a personalized, preventative approach to medicine. Pharmacogenomics. 2006 2006/01/01;7(1):5-9.

17. Diyabet 2020: Vizyon ve Hedefler - Türkiye [Diabetes 2020: Vision and Targets - Turkey]. Istanbul: Turkish Diabetes Foundation, under the auspices of the Turkish Ministry of Health, in collaboration with World Health Organization Regional Office for Europe and International Diabetes Foundation Europe, 2010.

18. Rose G. Sick Individuals and Sick Populations. Int J Epidemiol. 1985 March 1, 1985;14(1):32-8.

19. Rose G. The Strategy of Preventive Medicine. New York: Oxford University Press; 1994.

20. Manuel DG, Lim J, Tanuseputro P, Anderson GM, Alter DA, Laupacis A, et al. Revisiting Rose: strategies for reducing coronary heart disease. BM J. 2006;332(7542):659-62.

21. Zulman DM, Vijan S, Omenn GS, Hayward RA. The Relative Merits of Population-Based and Targeted Prevention Strategies. Milbank Q. 2008;86(4):557-80.

22. Frohlich KL, Potvin L. Transcending the Known in Public Health Practice: The Inequality Paradox: The Population Approach and Vulnerable Populations. Am J Public Health. 2008;98(2):21621.

23. Burton H, Sagoo GS, Pharoah P, Zimmern RL. Time to revisit Geoffrey Rose: strategies for prevention in the genomic era? Italian Journal of Public Health. 2012;9(4):e8665-1-9.

24. MCLaren L, Mclntyre L, Kirkpatrick S. Rose's population strategy of prevention need not increase social inequalities in health. Int J Epidemiol. 2010 April 1, 2010;39(2):372-7.

25. Frohlich $\mathrm{KL}$, Corin E, Potvin L. A theoretical proposal for the relationship between context and disease. Sociology of Health \& IIIness. 2001;23(6):776-97. 
26. Frohlich KL, Potvin L. Commentary: Structure or agency? The importance of both for addressing social inequalities in health. Int J Epidemiol. 2010 April 1, 2010;39(2):378-9.

27. Backholer K, Beauchamp A, Ball K, Turrell G, Martin J, Woods J, et al. A Framework for Evaluating the Impact of Obesity Prevention Strategies on Socioeconomic Inequalities in Weight. Am J Public Health. 2014 2014/10/01;104(10):e43-e50.

28. Turrell G, Oldenburg B, McGuffog I, Dent R. Socioeconomic determinants of health: towards a national research program and a policy and intervention agenda. Canberra, Australia: Queensland University of Technology, School of Public Health Research; 1999. Available from: http://eprints.qut.edu.au/585/1/turrell_health_inequalities.pdf.

29. Lorenc $T$, Petticrew $M$, Welch $V$, Tugwell $P$. What types of interventions generate inequalities? Evidence from systematic reviews. Journal of epidemiology and community health. 2012 August 8, 2012;2(67):190-3.

30. Sameroff A. A Unified Theory of Development: A Dialectic Integration of Nature and Nurture. Child Development. 2010;81(1):6-22.

31. The Future of the Public's Health in the 21st Century: The National Academies Press; 2003.

32. Policy Strategies: A Tobacco Control Guide. St. Louis: Center for Public Health Systems Science, George Warren Brown School of Social Work at Washington University in St. Louis and the Tobacco Control Legal Consortium; 2014.

33. Intergovernmental Committee on Drugs. National Tobacco Strategy 2012-2018: Commonwealth of Australia; 2012 [21 June 2015]. Available from: http://www.nationaldrugstrategy.gov.au/internet/drugstrategy/publishing.nsf/Content/ national_ts _2012_2018_html.

34. Simopoulos AP, Bourne PG, Faergeman O. Bellagio Report on Healthy Agriculture, Healthy Nutrition, Healthy People. Nutrients. 2013;5(2):411-23.

35. Simopoulos AP. The Impact of the Bellagio Report on Healthy Agriculture, Healthy Nutrition, Healthy People: Scientific and Policy Aspects and the International Network of Centers for Genetics, Nutrition and Fitness for Health. J Nutrigenet Nutrige. 2014;7(4-6):191-211.

36. Dobbs R, Sawers C, Thompson F, Manyika J, Woetzel J, Child P, et al. Overcoming obesity: An initial economic analysis - Discussion paper: McKinsey Global Institute; 2014. Available from: http://www.mckinsey.com/insights/economic_studies/how_the_world_could_better_fight_obesity.

37. From Commitments to Action: 'Second International Conference on Nutrition, Rome, 1921 November 2014, Conference Outcome Document: Framework for Action' [Conference Outcome Document]: Food and Agriculture Organization of the United Nations (FAO) and Word Health Organization (WHO); 2014. Available from: http://www.fao.org/3/a-mm215e.pdf.

38. Vogels AGC. Introduction and outline of this thesis. The identification by Dutch preventive child health care of children with psychosocial problems: do short questionnaires help? (PhD dissertation at Groningen University): University of Groningen; 2008. p. 5-18.

39. Syurina EV. Integrating personalised perspectives into Child and Youth Health Care: A long and winding road? (PhD dissertation at Maastricht University). 's-Hertogenbosch, the Netherlands: BOXPress; 2014.

40. Feigin VL, Norrving B. A new paradigm for primary prevention strategy in people with elevated risk of stroke. International Journal of Stroke. 2014;9(5):624-6.

41. Feigin VL, Wang W, Fu H, Liu L, Krishnamurthi R, Bhattacharjee R, et al. Primary stroke prevention in China - a new approach. Neurological Research. 2015;37(5):378-80. 
42. Gordon RS. An operational classification of disease prevention. Public health reports. 1983 Mar-Apr;98(2):107-9.

43. Busse R, Blümel M, Scheller-Kreinsen D, Zentner A. Strategies against chronic disease: what is being done? Tackling chronic disease in Europe: strategies, interventions and challenges. Copenhagen: World Health Organization, on behalf of the European Observatory on Health Systems and Policies; 2010.

44. Edwards N, Smith J, Rosen R. The primary care paradox: New designs and models: KPM G International; 2013. Available from:

http://www.kpmg.com/Global/en/IssuesAndlnsights/ArticlesPublications/primary-careparadox/Documents/primary-care-paradox.pdf.

45. Macinko J, Starfield B, Shi L. The contribution of primary care systems to health outcomes within Organization for Economic Cooperation and Development (OECD) countries, 1970-1998. Health Serv Res. 2003 Jun;38(3):831-65.

46. U.S. Health in International Perspective: Shorter Lives, Poorer Health. Washington, D.C.: The National Academies Press; 2013. Available from: http:// www.nap.edu/catalog/13497/us-healthin-international-perspective-shorter-lives-poorer-health.

47. The World Health Report 2008 - Primary Health Care Now More Than Ever. Geneva, Switzerland: World Health Organization; 2008. Available from: http://www.who.int/whr/2008/en/.

48. Western Pacific regional strategy for health systems based on the values of primary health care: World Health Organization, Western Pacific Region; 2010.

49. Transforming Primary Care: Safe, proactive, personalised care for those who need it most: Department of Health, UK; 2014. Available from:

https:// www.gov.uk/government/uploads/system/uploads/attachment_data/file/304139/Transfor ming_primary_care.pdf.

50. A strategy to prevent chronic disease in Europe - A focus on public health action: The CINDI vision. Copenhagen, Denmark: World Health Organization - Regional Office for Europe; 2004. Available from: http://www.euro.who.int/__data/assets/pdf_file/0010/134848/E83057.pdf.

51. Cox JL, Carr B, Vallis TM, Szpilfogel C, O'Neill BJ. A Novel Approach to Cardiovascular Health by Optimizing Risk Management (ANCHOR): A Primary Prevention Initiative Examining the Impact of Health Risk Factor Assessment and Management on Cardiac Wellness. Canadian Journal of Cardiology. 2011 11//;27(6):809-17.

52. Cox JL, Vallis TM, Pfammatter A, Szpilfogel C, Carr B, O'Neill BJ. A Novel Approach to Cardiovascular Health By Optimizing Risk Management (ANCHOR): Behavioural Modification in Primary Care Effectively Reduces Global Risk. Canadian Journal of Cardiology. 2013 11//;29(11):1400-7.

53. Badenbroek I, Stol D, Nielen M, Hollander M, Kraaijenhagen R, de Wit G, et al. Design of the INTEGRATE study: effectiveness and cost-effectiveness of a cardiometabolic risk assessment and treatment program integrated in primary care. BM C family practice. 2014;15(1):90.

54. van den Brekel-Dijkstra K, Rengers AH, Niessen MA, de Wit NJ, Kraaijenhagen RA. Personalized prevention approach with use of a web-based cardiovascular risk assessment with tailored lifestyle follow-up in primary care practice - a pilot study. European Journal of Preventive Cardiology. 2015 June 16, 2015.

55. Plochg T, Klazinga NS, Schoenstein M, Starfield B. Reconfiguring health professions in times of multimorbidity: Eight recommendations for change. 2011. In: Health Reform: Meeting the Challenge of Ageing and Multiple Morbidities [Internet]. OECD Publishing. Available from: http://dx.doi.org/10.1787/9789264122314-7-en. 
56. Cesuroglu T, Karaca S, Erge S. A practice model for personalized healthcare with a public health genomics perspective. Personalized Medicine. 2009;6(5):567-77.

57. Burnette R, Simmons LA, Snyderman R. Personalized health care as a pathway for the adoption of genomic medicine. Journal of personalized medicine. 2012 December;2(4):232-40.

58. Chang H-L, Shaw MJ, Lai F, Ko W-J, Ho Y-L, Chen H-S, et al. U-Health: an example of a highquality individualized healthcare service. Personalized M edicine. 2010 2010/11/01;7(6):677-87.

59. Vorderstrasse AA, Ginsburg GS, Kraus WE, Maldonado MC, Wolever RQ. Health coaching and genomics-potential avenues to elicit behavior change in those at risk for chronic disease: protocol for personalized medicine effectiveness study in air force primary care. Global advances in health and medicine : improving healthcare outcomes worldwide. $2013 \mathrm{M}$ ay;2(3):26-38.

60. Patel Cl, Sivadas A, Tabassum R, Preeprem T, Zhao J, Arafat D, et al. Whole genome sequencing in support of wellness and health maintenance. Genome M ed. 2013 Jun 27;5(6):58.

61. Kanodia AK, Kim I, Sturmberg JP. A personalized systems medicine approach to refractory rumination. Journal of evaluation in clinical practice. 2011 Jun;17(3):515-9.

62. Smarr L. Quantifying your body: a how-to guide from a systems biology perspective. Biotechnology journal. 2012 Aug; 7(8):980-91.

63. Craig P, Dieppe P, Macintyre S, Michie S, Nazareth I, Petticrew M. Developing and evaluating complex interventions: new guidance.: Medical Research Council; 2008. Available from: www.mrc.ac.uk/complexinterventionsguidance.

64. Moore GF, Audrey S, Barker M, Bond L, Bonell C, Hardeman W, et al. Process evaluation of complex interventions: M edical Research Council guidance. BMJ. 2015;350(h1258):1-7.

65. Moore G, Audrey S, Barker M, Bond L, Bonell C, Hardeman W, et al. Process Evaluation of Complex Interventions Guidance: UK Medical Research Council (MRC) guidance: MRC Population Health Science Research Network; 2014. Available from: http://www.populationhealthsciences.org/Process-Evaluation-Guidance.html.

66. Stafinski T, Menon D, Davis C, McCabe C. Role of centralized review processes for making reimbursement decisions on new health technologies in Europe. ClinicoEconomics and outcomes research : CEOR. 2011;3:117-86.

67. Towse A. Value based pricing, research and development, and patient access schemes. Will the United Kingdom get it right or wrong? British journal of clinical pharmacology. 2010 Sep;70(3):360-6.

68. Why Not the Best? Results from the National Scorecard on U.S. Health System Performance, 2011. The Commonwealth Fund, 2011 October 18, 2011. Report No.: 1500.

69. Murray CJL, Frenk J. Ranking 37th - Measuring the Performance of the U.S. Health Care System. New Engl J M ed. 2010;362(2):98-9.

70. Davis K, Stremikis K, Squires D, Schoen C. Mirror, M irror on the Wall, 2014 Update: How the U.S. Health Care System Compares Internationally: The Commonwealth Fund; 2014.

71. Hood L. A Doctor's Vision of the Future of Medicine. Newsweek. 2009 Jul 13, 2009.

72. Hood L, Price ND. Demystifying Disease, Democratizing Health Care. Sci Transl Med. 2014 February 26, 2014;6(225):225ed5.

73. Lukka K. What is a constructive research approach? [29.06.2015]. Available from: http://www.metodix.com/en/sisallys/01_menetelmat/02_metodiartikkelit/lukka_const_research_a pp/02_mita_konst_tut_tark. 
Declare the past, diagnose the present, foretell the future.

Hippocrates 
Appendix

\section{Additional information on}

Gentest 
This dissertation aimed to explore how a personalized health care model, such as Gentest, can be integrated into primary care services in European health systems for prevention of chronic diseases. Thus, the main question of the dissertation is 'how to integrate?', not 'if to integrate?'. It is indeed a crucial point to know if this practice model is worth integrating. However, evaluation of the content of Gentest wasn't covered in the scope of the dissertation, since it requires other research questions and a project that runs with dedicated funds. Moreover, it is not feasible to answer both questions at a single dissertation. The starting point of this dissertation was that Gentest had been piloted in Turkey and identified as a best practice model by Public Health Genomics European Network.

However, readers of this dissertation will probably have several questions raised in their minds about what Gentest is and how it has been implemented in Turkey. M ain properties and practice principles were already covered in Chapter 1 - Introduction and Chapter 6, the paper dedicated to Gentest. This appendix complements them with additional information in order to help the readers of this dissertation get a better picture on what Gentest is and what it is not. Particular attention was paid to cover the answers to the most frequently asked questions at scientific and technological platforms where Gentest was presented. (Main occasions where Gentest was presented were mentioned in Chapter 6 and a list of additional presentations is provided under 'List of publications and presentations' section).

This appendix contains two sections. First one is a summary of the timeline for development and implementation of Gentest. Then, a SWOT analysis for Gentest, which was originally carried out for another project, is presented.

\section{A summary of research, development and implementation history of Gentest}

GENAR Institute for Public Health and Genomics Research was founded by Serdar Savas, $M D$ and Gentest was conceived and developed under his leadership. The story of development of Gentest dates back to 1998-1999 when Dr. Savas was serving World Health Organization Europe as the Director for Programme Management / Deputy Regional Director in Copenhagen. During those years, he realized that developments in genetics could lead to solutions for the burden of chronic complex diseases. Then, he started monitoring scientific developments in genetics and exploring how these can be used for prevention of chronic diseases (idea development phase, 1998-199).

In 2000, Dr. Savas returned to Turkey and established BSS - United Health Systems consultancy, where he started planning for a center working on prevention of chronic diseases using genetic technologies, as well as inputs from other disciplines (planning phase). After the preparations, in 2004, it was established in Hacettepe University Science part as 
'GENAR', which was a privately funded research facility (small and medium size enterprise/ SME). Its first unit was 'GENAR Laboratory for biotechnology and Molecular Genetics'. At the time of its establishment, GENAR Laboratory was the highest throughput genotyping laboratory in Turkey. Later, GENAR Center for Nutrigenetics and Lifestyle Research and GENAR Center for Personalized Medicine and Pharmacogenetics joined to the Laboratory, and all became "GENAR Institute for Public Health and Genomics Research". In those years, GENAR was the third center working in Europe on the newly developing concept of public health genomics. The author of this dissertation, Tomris Cesuroglu, MD, served as the Research and Development Coordinator of GENAR Institute.

GENAR carried out various projects funded by national and international research and development funds, such as Framework Programme 6 of the European Commission and the Scientific and Technological Research Council of Turkey (TUBITAK). A major output of the research and development efforts of GENAR has been the practice model Gentest. It is a personalized health care practice that aimed to prevent common chronic diseases in primary care settings. It approaches health in a comprehensive manner and utilizes individuals' personal health information, detailed lifestyle analysis, body composition, genotype, and other biomarkers in order to prevent the onset or progression of major chronic diseases in a targeted way. [1, 2] (Research and development phase 2004-2008)

In 2008, Gentest was identified as a 'Best Practice Model' for public health genomics in Europe by the Public Health Genomics European Network (PHGEN I), based on the characteristics of the practice model being comprehensive (containing various factors such as personal, medical, lifestyle and genetic information), multidisciplinary, prevention orientated and implemented through health professionals [3]. It was piloted by authorized practitioners (physicians/or dieticians) as a service to 500 individuals in 2008-2009. At that period, the model was mainly practiced in the Gentest Implementation Center, which was run by GENAR. Genotyping was carried out at GENAR's high-throughput genotyping laboratories. After the pilot, designing and conducting a study that would collect data on the effectiveness of the model was envisaged, as conveyed in Chapter 6 . However, due to external conditions and constraints of the funding environment in Turkey, it was not possible to carry it out. (Pilot implementation phase 2008-2009)

After completion of the research and development phase of Gentest, GENAR has closed down its facilities at Hacettepe Science Park, as well as its Gentest implementation center in 2009. Since then, Gentest has been continued as a service provided through a number of authorized practitioners (physicians) in major cities in Turkey. Individuals who would like to get the Gentest pay for the service out-of-their pockets. The genotyping services for Gentest are purchased from a third party laboratory. (implementation phase since 2009)

Based on the current trends and developments in the world (such as demographic and epidemiologic transition and the need of health systems to reduce morbidity, mortality 
and costs related to complex diseases), feedback of scientific and industrial communities on the model, and past experiences with Gentest, it was identified that the main implementation area for such a preventive health care product would be wide spread population based approaches where Gentest is provided via primary care services in Europe.

Towards this vision, Tomris Cesuroglu received a M arie Curie Fellowship from the European Commission under Framework Programme 7. During her fellowship, she worked at the Institute for Public Health Genomics at Maastricht University to carry out the project "P4MEDICINE@EU" Evaluation of a Best Practice Model (Gentest) for Personalized Health Care within Public Health Genomics" (2011-2014). Within the P4-M EDICINE@EU project, among other tasks, she did an extensive literature review and carried out semi-structured interviews with various opinion leaders and experts around the world to explore opinions and topics regarding the implementation of personalized health care. This dissertation was carried out independent from the Marie Curie project and the interview data wasn't used for the articles of this dissertation. Nevertheless the overall fellowship process, including the literature review, the interviews and the conferences attended, helped her to gain valuable insights to the various topics in the field of personalized health care.

\section{SWOT analysis of Gentest}

Among the various tasks of the above mentioned Marie Curie project (P4-MEDICINE@EU), a SWOT analysis was carried out. This aimed to assess the strengths, weaknesses, opportunities and threats of/for the Gentest model from the perspective of its integration into Europe. In this this section, a modified version of the results of the SWOT analysis is presented. Many of these issues have already been reflected to different sections of the General Discussion of this dissertation (Chapter 8). Nevertheless, below analysis offers a concise overview of all strengths, weaknesses, opportunities and threats of/for Gentest in a single section.

The data source of the SWOT analysis was the publication on Gentest [2], sample questionnaire and report of Gentest, the proposal of the P4-MEDICINE@EU project, and internal and external documents of GENAR (such as business plans). In addition, the results of other tasks carried out under the P4-M EDICINE@EU project were considered.

\subsection{Strengths}

\section{- It is a health service model.}

Gentest is an integrative health service model, not a test. Being a health service model, it contains not only the 'tests' and 'assessments' (i.e. analysis) but also recommendations that include comprehensive lifestyle and follow-up plans designed for the needs of the individual (i.e. synthesis). Additionally, this service model contains tools to empower the 
health professionals to implement the model as well as manage the health of the individuals based on their health priorities.

- It is to be implemented inside health services, via trained health professionals.

The pilot in Turkey, as well as the envisaged implementation model in Europe (primary care setting), involves application of the model via trained health professionals (mainly physicians, as well as the dietitians). In the current practice in Turkey, the training for the health professionals is carried out in collaboration with Turkish Society of Public Health Genomics and Personalized Medicine (TOGEN). Only health professionals who complete the training successfully are authorized to become 'authorized practitioners of Gentest application'.

When compared with some direct-to-consumer applications, application via health professionals is an important strength.

- It is a comprehensive model that utilizes a large spectrum of inputs.

Gentest is based on the determinants of health model and aims to include relevant factors in all determinant areas as much as possible, including biological factors, lifestyle and environmental factors, socioeconomical factors and health systems. It makes a 360 degrees assessment of an individual using personal information (age, gender), detailed health information (personal health history including diseases and medications and family health history), living and working conditions, lifestyle information, including physical activity and exercise, smoking and drinking habits, supplement consumption, nutritional habits, food consumption ( $24 \mathrm{~h}$ recall and food frequency) using Gentest Food Portion Size Atlas (all these collected through a questionnaire (44 pages)); anthropometric (height, body weight, waist circumference), bioimpedance (body composition), pulse and blood pressure measurements; biomarkers (common markers such as LDL-, HDL- and total cholesterol, fasting plasma glucose, triglycerides, etc.); and genotype information (currently 65 polymorphisms).

- It is a model that assesses these inputs in an integrative way.

Gentest has an integrative approach which incorporates the above mentioned inputs for assessment of current status and development of personal medical follow-up and lifestyle plans. The assessments include, nutritional assessment (quantification of intake of all nutrients based on the information in the questionnaire and comparison with the recommended minimum and maximum intake levels), body composition assessment, assessment of physical activity and exercise (assessment of the current status and comparison with the recommended level), assessment of causes of smoking, assessment of current health information, genotype assessment, assessment of biomarkers, and assessment of the risks of major complex diseases: heart attack, stroke, type 2 diabetes, osteoporosis, and most common cancers (lung, breast, prostate, colon and stomach). 
- It has a holistic approach to health, by addressing major chronic complex diseases in a single service model.

Gentest covers major chronic complex diseases where lifestyle interventions play a very important role in development: cardiovascular diseases, diabetes, obesity, cancers and osteoporosis. Nevertheless, the lifestyle plan is expected to provide benefit in the whole spectrum of the health of the individual and has the potential to increase the quality of life.

There are programs on risk assessment and disease management of specific health areas in the world, such as cardiometabolic risk management programs or diabetes disease management programs. However, Gentest model has an inclusive approach to health to cover the broadest spectrum for managment of the health of the individual.

- It is a dynamic model which is open to addition of new types of inputs and assessments, as well as modification of prior ones based on new scientific developments.

Gentest is open to addition of new inputs, assessments, and recommendations, which can be derived from the scientific developments. It is not a static model. Developments can be incorporated to the model.

- It is a multidisciplinary approach.

Gentest aims to bring the knowledge from different disciplines in a way that complements each other. Multidisciplinary work is usually a very challenging task since the language, knowledge, skills and priorities of different disciplines vary, making it a complex task to deliver a multidisciplinary work. Gentest model has accomplished to combine the expertise from different disciplines, including medicine (as a whole and with its different specialties), nutrition \& dietetics, exercise physiology, epidemiology, (bio)informatics, life sciences (including genomics), behavioral sciences, public health, and others.

- It addresses a very important challenge in prevention of complex diseases: behavior change.

Currently, the best known prevention for complex diseases is adopting a healthy lifestyle. However, this is not achieved in many places of the world. Effective intervention models including lifestyle changes for prevention of these diseases is urgently needed. Gentest aims to address this need. Risks are identified individually, therefore Gentest creates personal vulnerability and risk perception and motivate individuals to implement the advices of the report. 
- It addresses a very important challenge in prevention of complex diseases: a holistic tool for the health professionals for management of the health of the individual according to health priorities.

Gentest empowers the health professionals (in particular primary care practices) by providing a holistic program on how to follow-up the individual based on their needs and health priorities. Thus, the primary care practice can have a program for each individual in his/her catchment area.

- Design and content of reports

The unique design of reports makes the report visually nice looking. Reports convey the message to the consumer in a simplistic way and also show how the results would be changed based on the behavior of the individuals to motivate the individual towards behavior change.

- Promoting personal autonomy in health

Gentest empowers the individuals with knowledge and tools to manage their health by making required lifestyle changes, and help them take the lead in management of their health.

On the other hand, the report might be perceived to restrict the lifestyle choices an individual can make (see 2.4. Threats, "Concerns on restriction of personal autonomy").

- Use of data collected for Gentest for research purposes.

The data collected for the implementation of the practice model can be used for research purposes as a feedback for further research and development. This data can be used to improve the algorithms of Gentest, making predictions and recommendations more accurate.

On the other hand, this use is also linked to a threat, which is ensuring compliance with the data protection legislations (see 2.4. Threats, "Data protection" in this appendix and the supplementary material of Chapter 7 )

\subsection{Weaknesses}

- Not scalable from the perspectives of production capacity and economic feasibility

Gentest reports are generated based on sets of algorithms and tools, however, these are not translated to a software. Reports are prepared by qualified human resources who can use these algorithms and tools. Preparation of each report requires time and effort of qualified labor. This limits the number of reports that can be generated per day and also increases the cost of the model. 
For the model to be implemented in a large scale in primary care services a software must be developed. This will reduce the costs as well as turnaround time. Additionally, as the volume increases, the cost of genotyping will decrease, making the model economically more feasible. (See also 2.3. Opportunities, "Development and use of software for collection of information, processing and reporting." and "Cost of genetic analysis is decreasing exponentially.")

- Challenges in assessment of nutritional status

Currently, assessment of nutritional status is carried out based on the reported consumption by each individual. This requires recall of food consumption by the individual (24 hours food consumption and food frequency questionnaire), which are dependent on the recall ability of the individual. Discoveries on biological markers of assessment of nutritional status would provide much more robust results. (See also 2.3. Opportunities, "Use of biomarkers for assessment of nutritional status") Also, development of smart phone apps using the food portion size atlas or use of existing apps for collection of 24-hour food consumption data is an important opportunity.

\section{- Data collection consumes time.}

Gentest is a comprehensive model, which includes personal information, health information, living and working conditions, lifestyle information (including very detailed food consumption information) using a questionnaire (see 2.1. Strengths, "It is a comprehensive model that utilizes a large spectrum of inputs."). Collection of data and information consumes considerable amount of time (around 1 hour) of the health professional or his/her trained assistant, as well as the individual.

Putting the questionnaire into a software in a way that can be filled by the individual more rapidly would decrease related time and costs. (See also 2.3. Opportunities, "Development and use of software for collection of information, processing and reporting.") Also, prioritization can be done on the extent of the analysis and thus the type and amount of the data to be collected.

\section{- Effectiveness and cost-effectiveness not demonstrated yet}

The effectiveness and cost-effectiveness of the model hasn't been demonstrated yet.

The effectiveness and cost effectiveness are closely related to the context where the implementation will take place (see also Chapter 8 - General Discussion). Therefore, studies on effectiveness and cost-effectiveness of the model must take place in the setting where the model will eventually be implemented. 
The following are update and further development areas that are currently not present in the model. Their addition will improve the model significantly.

- Genetic markers

As scientific publications provide new insights to genetic basis of diseases and response to lifestyle factors, the genetic markers (currently polymorphisms) used in Gentest need to be updated as well. An update on the list of polymorphisms is currently underway, which is envisaged to be completed by the end of 2015. Newly developing opportunities on other biological markers such as epigenetics, expression profiling and metabolomics should also be explored in the following periods.

- Assessment of functional physical fitness is not present.

Currently, the model assesses the physical activity and exercise status of the individuals based on the type, duration and intensity of the exercises they report, in six physical fitness areas. Actual fitness status is not assessed and this should be added to provide a more comprehensive picture on the individual. For measurement of physical fitness, measurement of VO2max for cardiorespiratory fitness (by measuring oxygen consumption during exercise in a controlled clinical environment); measurement of hand grip as an indication of muscle strength; and tests for measurement of flexibility can be used.

- Basal metabolic rate measurement is not present.

The basal metabolic rate that is used for calculation of recommended calorie intake and macronutrients is currently estimated with calculations, not measured. Measurement of basal metabolic rate in controlled environment (or resting metabolic rate) would enable the results be more personalized.

- Assessments of psychological and functional status are not present.

The psychological aspects of health are not currently included in the model. Mental health should be included in the model with appropriate questionnaires. In particular, assessment of stress levels should be made and algorithms for stress reduction recommendations should be developed.

For an overall assessment of functional physical and mental health, a questionnaire such as the Short Form 36 Health Survey can be incorporated (it includes following scales: physical functioning, physical role, bodily pain, general health, vitality, social functioning, emotional role, mental health). 
Before implementing to new countries adaption is needed in following areas:

- Methodology for assessment of food consumption needs to be adapted for each country.

The current food consumption questionnaire and the food portion size atlas are developed for Turkey. These need to be adapted for each country (or groups of countries) that are targeted for implementation.

In addition, currently an international food composition database is used. For each new country (or groups of countries) their own food composition database (if present) must be used for calculation of nutrient intakes. (see also Chapter 8 - General Discussion).

- Risk assessment algorithms need to be adapted for each country. Currently, international risk assessment algorithms are used. However, accuracy of risk assessment algorithms varies from country to country. Therefore, if existing, risk assessment algorithms of the target country must be adopted in the model. (see also Chapter 8 General Discussion).

- Medical, nutritional and other algorithms need to be adapted for each country, if national consensus guidelines exist in those areas.

Currently, international and Turkish guidelines are used in the model. If the target country has national guidelines, the algorithms on medical assessment and recommendations, nutritional assessment and recommendations, and others need to be adapted to be in line with them. (see also Chapter 8 - General Discussion).

- The reports must be adapted to the health literacy level of the target population.

Current report targets people with medium health literacy. The reports must be adapted to different health literacy levels of the target population. This should be done both in country level and for different socioeconomic levels in a country (as an indicator of health literacy). Different report templates may need to be produced for targeting different socioeconomic levels. (see also Chapter 8 - General Discussion).

\subsection{Opportunities}

- Scientific developments in the whole spectrum of genome-based knowledge and technologies.

At the time of the development of Gentest model, the most prominent markers on development of complex diseases were polymorphisms. Due to scientific developments, new parameters may be derived from the whole spectrum of omics, i.e. epigenetics, gene expression, proteomics and metabolomics. Their incorporation to Gentest model provides an opportunity. 
- Development and use of software for collection of information, processing and reporting.

When a software is developed for the model, it will be possible to collect information, process and generate the Gentest report using the software. This will not only increase the production capacity, but also lower the costs.

A major challenge for development of a software for this model is its dynamic content (see 2.1. Strengths, "It is a dynamic model which is open to addition of new types of inputs and assessments, as well as modification of prior ones based on new scientific developments."). The developed software should also allow the model to be dynamically updated. As software development approaches and methods improve in time, this need will be better met.

- Provision of the model to the whole world via internet.

By using a web portal, the components (such as food consumption methods including the food portion size atlases) or the whole of the model can be provided as a service to the whole world.

- Cost of genetic analysis is decreasing exponentially.

The cost of genetic analysis has been decreasing rapidly. This provides an important opportunity for Gentest to decrease the costs while increasing the number of genetic markers.

- Use of whole genome/ exome sequencing in health care.

The cost of sequencing a whole genome has been decreasing exponentially, in particular after the development of second generation DNA sequencing technologies in 2008 [4]. The use of whole genome or exome sequencing (WGS/WES) in newborn screening is also debated. Wide use of WGS/WES has the opportunity to include an enormous amount of genetic information in the model.

UK prime minister announced in December 2012 their plans to carry out whole genome sequencing in 100.000 patients with cancers and rare diseases within UK's National Health Services. This indicates that decision makers can be willing to use genetic information in the mainstream of the health services, and provides an additional opportunity for the future. [5]

- Use of biomarkers for assessment of nutritional status

The scientific developments in the fields of proteomics and metabolomics are expected to allow assessment of nutritional status, as well as other lifestyle factors, using biomarkers. This will help to standardize these assessments and help create more robust results for nutritional assessments. 


\section{- The pressure for innovation in health}

Health systems are under pressure to find solutions to the problems of the aging population and ever increasing burden of chronic complex diseases which require long term care. Innovation is addressed as one of the key solutions to approach these problems effectively.[6] "...But now and for the foreseeable future we must meet these demands from within our current real terms funding, while at the same time improving quality. This means that simply doing more of what we have always done is no longer an option. We need to do things differently. We need to radically transform the way we deliver services. Innovation is the way - the only way - we can meet these challenges. Innovation must become core business for the NHS"[7]. (see also Chapter 8 - General Discussion)

Gentest is an innovation addressing the major challenges in prevention of complex diseases, i.e. behavior change and management of health together with the health professional. It is an opportunity for Gentest to be implemented in an environment where decision makers have understood the need for innovation effectively. (see 2.4. Threats, "Resistance to change the current paradigm in health care services" in this chapter and Chapter 8 - General Discussion)

\section{- The pressure for changing the current paradigm in health services}

Due to the same reasons laid down in the previous item (The pressure for innovation in health) there is a need for paradigm shift in health care services. Currently, the services are reactive, disease oriented, focused on acute and medical services. However, to tackle with the challenge of complex diseases, the services should be proactive, health oriented, focused on chronic cure and care covering the whole spectrum of health care, not only medicine.

Gentest model targets health care services within this shifted paradigm. If the need for the shift is well understood, it will provide a very positive environment for the implementation of Gentest. If the need for the paradigm shift is not in the agenda of the health system of the country, or overall Europe, it is very difficult to implement Gentest (see 2.4. Threats, "Resistance to change the current paradigm in health care services" in this chapter and Chapter 8 - General Discussion).

\section{- Use in other countries}

Gentest model can be adapted and implemented in various other countries, as it is the subject to this dissertation is to investigate how it can be integrated into countries in Europe. (For some specific aspects on adaptation to new countries, see 2.2. Weaknesses "Before implementing to new countries adaption is needed in following areas:") 
- The trends of incorporating risk assessment and management programs into primary care services

In the recent years, increasing number of risk assessment and management programs for primary care services and/or primary prevention are being developed and reported in the literature [8-13]. This validates that it is a timely and valid case to introduce Gentest into primary care services.

\subsection{Threats}

- Potential misuse by health insurances and employers

There is a potential that Gentest report can be misused by health insurances and current and potential employers. Legislative precautions must be in place to provide security to the individuals.

- Potential misuse within the family

There is a potential that Gentest can be misused in the family context. Genotype data can be used to assess or verify the biological parental relationships. The risk profile and recommended lifestyle changes can create pressure on family members, or the family members can create pressure on the individuals.

It is not possible to address these points by legislations. Other methods must be found to minimize threats that may come from them.

\section{- Data protection}

The Data Protection Directive (Directive 95/46/EC on the protection of individuals with regard to the processing of personal data and on the free movement of such data) provides the general framework on data protection issues in EU countries. However, legal and administrative requirements for data protection vary in practice among EU countries. This creates a challenge since Gentest must comply with the regulatory and administrative framework of each country individually.

A proposal for General Data Protection Regulation was released in 2012 to unify data protection within EU. The adoption is aimed for in 2014 and it is planned that the regulation takes effect in 2016. However, discussions on the content of the proposed regulations still continue. Use of personal data for unspecified future research purposes is a major topic debated under data protection issues. (See also 2.1. Strengths, "Use of data collected for Gentest for research purposes." In this appendix and the supplementary material in Chapter 7)

\section{- Security of the data stored}

When Gentest is implemented on wide populations, an enormous size of data will be generated for all enrolled individuals. This data needs to be securely stored, which creates a 
challenge, in particular from the perspective of protection of unauthorized access to the data.

- $\quad$ Effects on health disparities

There is a possibility that uptake of the messages of Gentest report might be better in higher socio-economical status (SES) groups and worse for lower SES groups. This may increase the health disparities among different groups. This must be addressed by adaption of the reports to different health literacy levels within the target populations (see also 2.2. Weaknesses, "The reports must be adapted to the health literacy level of the target population.")

- Limited knowledge and skills of health professionals on genomics

The knowledge and skills of the health workforce (physicians including general practitioners, nurses, dietitians, etc.) on genomics is limited. Health professionals may have difficulty in comprehending the role of genomics in this model. Therefore, appropriate training programs for health professionals must be developed.

\section{- Possible concerns of the primary care workforce}

Even if data collection is streamlined using a software, it will still consume time (of practice nurse and/or physician assistant). Additionally, the report must be explained to the individual by a health professional (physician, practice nurse and dietitian). These are likely to be major concerns of the workforce for implementation of the model, in particular considering the current organization of the family physician settings and their work load. (See also the supplementary material in Chapter 7)

One solution can be prioritization of different components of the model based on the potential benefit they provide and the time they consume. In this approach, only the components that have high added benefit when compared to the time they consume would be included and components with less added value (compared to the time they consume) would be left out.

- $\quad$ Resistance to change the current paradigm in health care services

Currently, the health care services are reactive, disease oriented, focused on acute and medical services. However, to tackle with the challenge of complex diseases, the services should be proactive, health oriented, focused on chronic cure and care covering the whole spectrum of health care, not only medicine. (see also 2.3. Opportunities, "The pressure for changing the current paradigm in health services" in this appendix and the supplementary material of Chapter 7)

Gentest model targets health care services within this shifted paradigm. Successful implementation of the model requires shifting the mindsets of the players within a health system and, which is a serious challenge. It will be very difficult to implement Gentest in 
an environment where players have a resistance for changing their paradigm on health care services.

\section{- Concerns on restriction of personal autonomy}

The model is envisaged to promote the personal autonomy of individuals to manage their health (see 2.1. Strengths, "Promoting personal autonomy in health"). However, there is also a possibility that the report might be perceived to restrict the lifestyle choices an individual can make, i.e. "being told what to do". On the other hand, the individual is empowered by the information she/he gains from the report, and it is up to his/her free will to implement it or not.

\section{- Competition with other applications}

Under 2.3. Opportunities, it was reported that increasing number of risk assessment and management programs for primary care services are being developed and reported in the literature [8-13]. This may create a competition for Gentest. Nevertheless, it should be noted that the reported programs are vertical one which focus on a single disease/ health area (cardiovascular/ cardiometabolic risk/health), whereas Gentest integrates lifestyle interventions for major chronic diseases that create the biggest burden of disease on the society.

\subsection{Overview}

Overall, Gentest provide a very different profile than commonly known consumer genomics products which use genetic information to make 'personalized' recommendations, such as 23andMe. First of all, unlike 23andMe, Gentest is not a 'test'; it is a service model. Moreover, it is provided via health professionals, not directly to consumer. Finally, it is not a service focused on genetics. It is a comprehensive approach to health and genetics is one of the lines of the inputs. These properties indicate that Gentest is not comparable to consumer genomics products such as 23 andM e.

Overall, addressing major chronic complex diseases in a single test combining genetic information, anthropometric and lifestyle factors etc., and potentially empowering individuals to manage their health are among the main strengths of Gentest. Lack of effectiveness/ cost-effectiveness data seems to be its main weakness. Some ideas on how the practice model can be evaluated were presented in Chapter 8 (General Discussion). The areas which need to be updated and/ or adapted to target countries can be regarded as both weaknesses and opportunities for further improvement. One of the main opportunities is development of a software to collect information and generate 'Gentest' reports. Also, data generated with its potential population wide application in primary care can create a feedback loop to research providing valuable insights to health and diseases. $A$ main threat is the limited knowledge and skills of the health workforce on genomics and its contribution to more targeted health interventions.

An overview of the SWOT analysis is presented in Figure 1. 


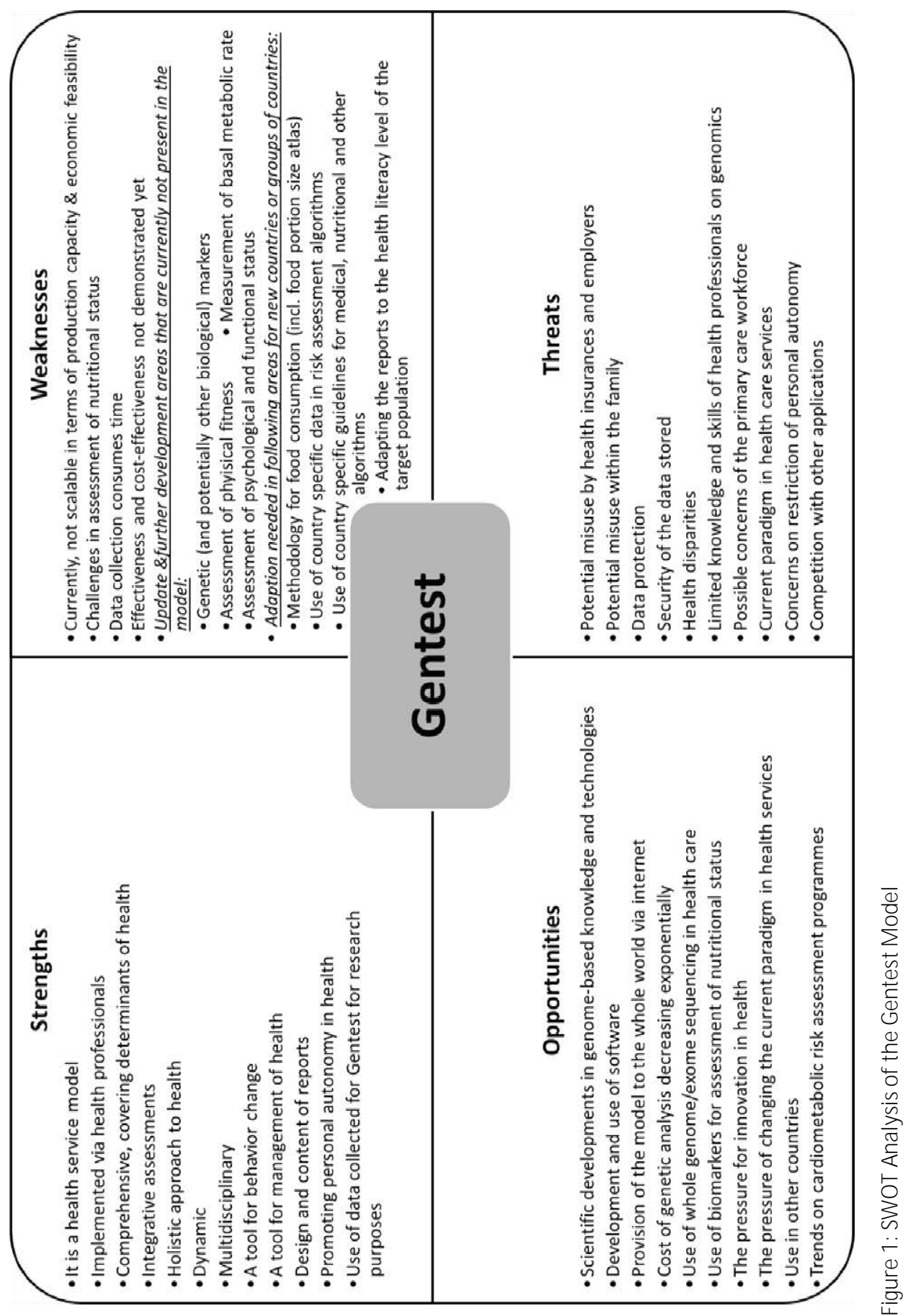




\section{References}

1. Savas B. A Case in Commercial Applications of Nutrigenetics. Abstract A31. In: 1st Congress of the International Society of Nutrigenetics/Nutrigenomics (ISNN). J Nutrigenet Nutrigenomics. 2007;1(1-2):72.

2. Cesuroglu T, Karaca S, Erge S. A practice model for personalized healthcare with a public health genomics perspective. Personalized M edicine. 2009;6(5):567-77.

3. P4Medicine@EU - Evaluation of a Best Practice Model for Personalized Health Care within Public Health Genomics - Final report summary (Project no: 272563, Funded under FP7-PEOPLE): European Commission - CORDIS; 2013. Available from: http://cordis.europa.eu/projects/rcn/98922_en.html.

4. Wetterstrand K. DNA Sequencing Costs: Data from the NHGRI Genome Sequencing Program (GSP): National Human Genome Research Institute (NHGRI), NIH; [updated July 16, 2013; cited 2013 28.09.2013]. Available from: www.genome.gov/ sequencingcosts.

5. Cressey D. Nature News Blog [Internet]: Nature Publishing Group. 2012. [cited 2013]. Available from: http://blogs.nature.com/news/2012/12/uk-pushes-whole-genomesequencing-into-clinical-practice.html.

6. $\quad$ Steering Group on Strategic Implementation Plan for the European Innovation Partnership on Active and Healthy Ageing. Stratgic Plan 2011. Available from: http://ec.europa.eu/research/innovation-union/pdf/active-healthy-ageing/steeringgroup/implementation_plan.pdf.

7. Department of Health UK. Innovation, Health and Wealth, Accelerating Adoption and Diffusion in the NHS. UK: Department of Health; 2011. $34 \mathrm{p.}$

8. Cox JL, Carr B, Vallis TM, Szpilfogel C, O'Neill BJ. A Novel Approach to Cardiovascular Health by Optimizing Risk Management (ANCHOR): A Primary Prevention Initiative Examining the Impact of Health Risk Factor Assessment and Management on Cardiac Wellness. Canadian Journal of Cardiology. 2011 11//;27(6):809-17.

9. Cox JL, Vallis TM, Pfammatter A, Szpilfogel C, Carr B, O'Neill BJ. A Novel Approach to Cardiovascular Health By Optimizing Risk Management (ANCHOR): Behavioural Modification in Primary Care Effectively Reduces Global Risk. Canadian Journal of Cardiology. 2013 11//;29(11):1400-7.

10. Badenbroek I, Stol D, Nielen M, Hollander M, Kraaijenhagen R, de Wit G, et al. Design of the INTEGRATE study: effectiveness and cost-effectiveness of a cardiometabolic risk assessment and treatment program integrated in primary care. BMC family practice. 2014;15(1):90.

11. van den Brekel-Dijkstra K, Rengers AH, Niessen MA, de Wit NJ, Kraaijenhagen RA. Personalized prevention approach with use of a web-based cardiovascular risk assessment with tailored lifestyle follow-up in primary care practice - a pilot study. European Journal of Preventive Cardiology. 2015 June 16, 2015.

12. Feigin VL, Norrving B. A new paradigm for primary prevention strategy in people with elevated risk of stroke. International Journal of Stroke. 2014;9(5):624-6.

13. Feigin VL, Wang W, Fu H, Liu L, Krishnamurthi R, Bhattacharjee R, et al. Primary stroke prevention in China - a new approach. Neurological Research. 2015;37(5):378-80. 
This universe is not outside of you.

Look inside yourself; everything that you want, you are already that.

M evlana Rumi 
Executive Summary

Samenvatting | Özet 


\section{Summary}

Chronic complex diseases create a huge burden and economic toll on health systems and societies. Driven by scientific and technological developments, personalized medicine and personalized health care have a potential to provide solutions for effective prevention of chronic diseases. However, integration of 'personalized' practices into health services has been a challenging process.

This dissertation used a preventive personalized health care model called 'Gentest' as a practical example to address the integration issues effectively. Studying how it can be integrated into health systems helped us to identify directions required to assess and address integration issues effectively, as well as drawing lessons for other 'personalized' practices. Thus, the aim of this dissertation has been to see how a personalized health care model, such as Gentest, can be integrated into primary care services in the European health systems, for prevention of chronic diseases. To answer that, three sub-questions were asked: 1 . Where will the practice model be integrated?; 2 . What will be integrated?; and 3. How can the integration take place?

The dissertation took a journey through the fields of personalized medicine and health care, and health systems and policies, using Gentest as its vehicle. Part I answered 'where' the integration will take place and described the setting, i.e. health system in its wider context of public health. Part II answered the 'what' question by first describing the broader context of personalized medicine and health care and then introducing Gentest as an example of a practice model to be integrated into the European health systems. Part III presented a systematic approach to assess 'how' this practice model can be integrated into primary care services around Europe. It provided an inventory of areas and issues that can be involved in the integration process of the practice model. More specifically, the dissertation journey provided the following chapters as its output.

Chapter 1 showed an urgent need to develop solutions to reduce the burden of chronic complex diseases that reached an epidemic level globally. To achieve that, health systems need a paradigm shift towards a more proactive, health oriented and preventive services that are better focused on chronic cure and care covering the whole spectrum of health care (not only medicine). Personalized health care practices can provide important opportunities to facilitate this shift and contribute to prevention of chronic complex diseases. The example we used is Gentest, a practice model which provides a comprehensive approach to major chronic diseases and related risk factors to prevent development and progression of diseases in a targeted way. Upon its pilot in Turkey and having been identified as a 'best practice model' in Europe, it was identified that the main implementation area for such a preventive intervention should be primary care services in Europe, where it can be provided via general practitioners/ family physicians. However, there has been no successful example of integration of such a preventive personalized health care model 
provided to large populations. Therefore, we needed to explore how to integrate Gentest into primary care services in Europe. This would be done by answering the where, what and how questions, as laid above.

Part I aimed to describe the setting where the practice model will be integrated into. It was seen that the whole debate on where to integrate needed to build on the concepts of 'public health' and 'health systems'. However, there is confusion around these concepts, and how they are interrelated. As a solution, in Chapter 2, we took the endeavor to integrate concepts of public health, including health system and its components, into a single coherent framework called 'Health Globe'. This served to the purpose of achieving a coherent terminology for policy makers and those who guide them internationally. Health Globe uses the World Health Organization's 'Health for All' vision, introduced in 1977, as its overarching framework. The broad approach to health brought by 'Health for All' was defined as public health. Thus, all activities that directly or indirectly affect individuals' health, should fall within the scope of 'public health'. This includes medical and nonmedical interventions, governmental decisions and practices, and activities of the public sector, the private sector and NGOs. Health system is a part of the Health Globe. It consists of activities under the mandate of the ministry of health. As a result of this exercise, a framework for the objectives and functions of a health system was introduced. This included a description of different layers of health services. The specific setting where our practice model will be integrated into is primary care services. Therefore, within the description of health services, the scope and position of the primary care services was clearly depicted.

The USA has a leading position regarding development of the concepts and practices of personalized medicine and health care, as most of the existing examples and publications are originating from there. Chapter $\mathbf{3}$ investigated the U.S. health system to understand the context of these 'personalized' practices and learn lessons from their situation when discussing how to and how not to integrate personalized practices in Europe. This chapter showed that what USA has is hardly a 'system' designed in a coherent way. Moreover, in terms of policy, USA has prioritized 'freedom' and 'free market' over 'equity' as policy values. These have led to serious shortcomings in terms of the main outcomes of a health system, i.e. effectiveness, efficiency and equity. After conveying the problems and underlying reasons, this chapter proposed how to develop American health policy and health system to increase its effectiveness and efficiency, while reducing inequity. The recommended solutions were heavily drawn on the experiences of European countries.

Part II of the dissertation aimed to explore what will be integrated by describing the broad context of personalized medicine and health care, as well as our specific case example Gentest. Chapter 4 showed that the future vision of personalization of medicine and health care cannot be uniformly defined using standard definitions. M oreover, the terms used now have been defined in a top-down manner by influential people or committees 
and may not adequately reflect the real-life field. Chapter 4 provided a different angle with a 'practice-based perspective' (or a 'bottom-up perspective') to the debate by analyzing the content of 'personalized' practices published in the literature. These practices were identified with a systematic search in the scientific literature. The content analysis indicated that the definition of the personalized approaches is problematic and the 'personalized' practices are not a homogeneous group. In terms of the application form and context, a 'personalized' practice can be a commodity in health care market, a way how health services are provided, or a keyword for emerging applications. A 'personalized' practice can serve various groups for different purposes: It can help individuals/patients to empower them to gain control of their health, health professionals to provide better services, health care organizations to increase effectiveness and efficiency of health care delivery in the competitive environment, or national health systems to increase performance in terms of not only effectiveness and efficiency but also equity. Additionally, using three country examples (USA, France and Taiwan), it was identified that for successful integration of the practices into health services, not only the attitude of the country towards innovations, but also the health system and policies context is crucially important.

Chapter 4 showed the various facets of the personalized approaches but it was Chapter $\mathbf{5}$ where a working definition of personalized health care was made. The article clarified what commonly used terms in the field, such as personalized medicine, personalized health care and individualized medicine, mean to their authors. Initially, 'personalized medicine' was considered as equal to 'genomic medicine'. However, our genome is not the only factor that makes us unique. Individuals must be considered in their entire context, including exposure to pathogens or toxins, socioeconomic factors, physical activity, nutrition and other lifestyle factors, which are constantly interacting with each other, as well as with the genomes. Thus the article called for an integrative approach covering various determinants of health for both the scientific discoveries and the personalized approaches in practice. In its conclusion, it urged to prepare health systems and policy makers in a timely manner for the paradigm shift towards the personalized approaches.

Having set the scene of personalized medicine and health care in Chapters 4 and 5, Chapter 6 provided a concrete example of personalized health care used in this dissertation: Gentest. It is a comprehensive and integrative model of personalized health care for prevention of common chronic diseases. It utilizes individuals' personal health information, detailed lifestyle analysis, body composition, genotype, and other biomarkers in order to prevent the onset or progression of major chronic diseases in a targeted way. The mission of this practice model is changing the behavior and managing the health of individuals according to their health priorities. It was developed by GENAR Institute for Public Health and Genomics Research in Ankara, Turkey. The article conveyed how the (pilot) implementation was provided to over 500 individuals via authorized practitioners, i.e. health 
professionals (mainly physicians) who completed a training programme for this purpose. The positive and negative criticisms towards this practice model were also briefly covered.

After Part I, which described the setting, i.e. health systems, and Part II, which described the personalized preventive practice model in its wider context of personalized health care, Part III explored how the practice model can be integrated into the into the setting, i.e. health system.

If we want to integrate models such as Gentest into primary care services in Europe, what are the issues that we should investigate? How can we approach this in a systematic and analytical way? What areas of the health system of the country should be investigated? Chapter 7 aimed to answer these questions. To identify all relevant integration issues, a systematic approach was developed using the health systems framework, which was introduced in Chapter 2. Thus, the functions of the health system provided the overarching framework to this systematic approach. As was shown in Chapter 4, 'personalized' practices are diverse and cannot be covered with a generic approach. Therefore, Gentest was used in this exercise for identification of the issues to be covered. As a result, 24 areas were compiled to be assessed under the functions of health systems. The application of the systematic approach was enriched with examples provided from the Netherlands and the UK, as well as other European settings. A prioritization among these 24 areas were made to identify which ones are crucial and with high priority when a practice model is integrated into a health system. In general, Chapter 7 conveyed a systematic approach to identify and assess issues related to integration of the practice model into primary care services in European health systems. Since this method can be applied to other practices and countries, it provides a solution to the broader problem of the integration of personalized medicine and health care practices into health systems.

Chapter 8 took a step back and reflected on the interaction between the contexts of health systems and personalized health care practices. While setting the scene, it was conveyed that the Health for All vision, born in 1977, eventually led to development of ecological approaches to health, which emphasize the importance of understanding the bio-psycho-socio-ecological context of individuals. As each individual has slightly differing contexts, understanding the individual context and designing interventions that are tailored/ personalized to it provide an important opportunity to approach health in a comprehensive way. Moreover, with integrative personalized health care models that address health, risks and diseases of individuals holistically, such as Gentest, we may progress towards attaining the goal of 'Health for All'. However, in order to host such practices effectively, as well as to attain the 'Health for All' goal, health systems need to innovate to shift towards proactive and health oriented services focused on prevention as well as chronic cure and care, covering the whole spectrum of health care (not only medicine). This also calls for revisiting conventional concepts in public health, such as the dichotomy of 'population based vs. high risk individual'. We need to move towards a preventive par- 
adigm which understands that personalized approaches may help to target the population with individual level interventions. Moreover, considering the continuum of development of chronic diseases, 'personal health management', 'personal risk management' and 'personal disease management' can be considered as a new classification of preventive interventions. Another important area of innovation is strengthening the primary care services so that they can provide such preventive and personalized approaches to the population in a proactive way. The practice models for personalized health care, such as Gentest, may also be used as a practical opportunity to realize the paradigm shift within the health systems.

Those innovations on the side of health systems must be coupled by efforts to innovate the personalized health care practices. First of all, innovation is needed to bring a comprehensive approach to so-called 'personalized' practices. Can we regard practices that use a single test, whose result leads to a single intervention for diagnosis or therapy of a disease, as a 'personalized'? 'Personalization' can only be achieved when we understand and use the individual contexts in a holistic way, rather than focusing on a single factor, be it high-tech or low-tech, biological or non-biological. Moreover, to adapt to the target countries and health systems, personalized health care practices, such as Gentest, need to continue to innovate. Eventually, the field of personalized medicine and health care needs to realize that the discoveries they are making and technologies and tools they are developing cannot have a profound effect on the society unless a comprehensive approach to their integration into health systems is developed.

In terms of methodological considerations, Chapter 8, reflected on the usefulness of using a concrete, real life case in this dissertation and how it may contribute to research in this field.

Chapter 8 ended with listing the main conclusions derived from this dissertation. Very briefly, it was shown that personalized health care offers valuable opportunities to combat the increasing burden of chronic complex diseases while serving to the required paradigm shift. To achieve this shift and to attain the goal of 'Health for All', innovations for personalized medicine and health care, and health systems and policies need to co-evolve, adapting themselves to the needs of each other while leveraging one another. For successful integration of personalized health care practices, issues related to integration need to be approached systematically, not in a piecemeal way. Health system frameworks can provide the required analytical frameworks to identify and assess issues related to integration of those practices. An effective dialog among the fields of personalized medicine and health care and health systems and policies may facilitate this integration process to be better and faster. 


\section{Samenvatting}

Complexe, chronische ziekten veroorzaken een behoorlijke belasting voor en economische druk op onze gezondheidssector en de maatschappij. Vanwege de wetenschappelijke en technologische ontwikkelingen heeft 'personalized medicine/ personalized health care' een hoog potentieel om deze complexe, chronische ziekten te bestrijden. 'Personalized medicine/ personalized health care', wat vertaald kan worden als 'gepersonaliseerde geneeskunde/ gepersonaliseerde gezondheidszorg', kan gezien worden als behandeling / zorg die afgestemd is op de specifieke karakteristieken van de patiënt. Gepersonaliseerde geneeskunde/ gepersonaliseerde zorg is veelbelovend en biedt nieuwe uitdagingen voor de praktijk.

Aan de hand van het gezondheidsmodel Gentest wordt in dit proefschrift beschreven hoe integratie van gepersonaliseerde zorg geëffectueerd kan worden respectievelijk wat wij kunnen leren van de praktijk van gepersonaliseerde zorg. Het doel van dit proefschrift is om te kijken hoe een gepersonaliseerd model, zoals Gentest, kan worden geïntegreerd in Europese gezondheidssystemen. Om deze vraag te beantwoorden wordt er gekeken naar drie sub-vragen: 1 . Waar zal het model worden geïntegreerd?; 2 . Wat zal er worden geïntegreerd?; 3. Hoe kan de integratie tot stand komen?

In Deel I van het proefschrift wordt besproken 'waar' integratie van gepersonaliseerde zorg plaats zal vinden en de samenhang binnen de bredere context van publieke gezondheid. In Deel II wordt uitgelegd 'wat' gepersonaliseerde zorg precies inhoudt en met Gentest als voorbeeld. Ten slotte wordt in Deel III uiteengezet 'hoe' dit model in de eerstelijns zorg binnen Europa kan worden geïntegreerd. Verder is het proefschrift verdeeld in hoofdstukken waarin de verschillende onderwerpen meer in detail worden besproken.

Hoofdstuk 1 toont de noodzaak tot oplossingen voor de belasting die chronische ziekten leggen op onze maatschappij. Om chronische ziekten in een eerder stadium aan te kunnen pakken, is er een paradigmaverschuiving in de gezondheidszorg vereist. Tegenwoordig wordt er nog veel nadruk gelegd op curatie (het genezen van mensen die al ziek zijn) binnen de gezondheidszorg, maar, in het continuüm tussen vroeg preventief en hoog gespecialiseerde curatieve zorg, zou meer accent moeten liggen op vroeg preventief. In dit opzicht kan gepersonaliseerde zorg een grote rol spelen. Gentest is hierbij als voorbeeld gebruikt. Gentest is een praktijk model dat focust op de belangrijkste complexe, chronische ziektebeelden. Hierbij kunnen er aan de hand van risicotaxaties uitspraken worden gedaan over de kans op een bepaalde ziekte en kan er worden geprobeerd te voorkomen dat deze mensen ziek worden door ze persoonlijke geneeskunde aan te bieden. Gedurende de proefperiode van Gentest in Turkije is het uitgeroepen als het 'beste praktijk model' in Europa. Omdat er nog echter geen soortgelijke modellen zijn geïntegreerd in Europa, was het belangrijk om uit te zoeken hoe een integratie van een model als Gentest kan 
plaatsvinden en welke facetten hierbij een rol speelt. Om dit uit te zoeken zijn de 'waar', 'wat' en 'hoe' vragen gebruikt.

In deel I wordt de omgeving besproken waarin Gentest geïntegreerd kan worden binnen de bredere context van 'public health' (publieke gezondheid) en 'health systems' (zorgstelsels). In hoofdstuk 2 wordt een samenhangend kader gepresenteerd, 'Health Globe' (Wereldbol van de Gezondheid), om meer eenheid van taal te bevorderen voor beleidsmakers in de gezondheidszorg. Hierdoor wordt een coherente terminologie voor beleidsmakers op internationaal niveau toegankelijker gemaakt. De 'Health Globe' maakt gebruik van de visie 'Gezondheid voor iedereen' en werd geïntroduceerd in 1977 door de Wereldgezondheidsorganisatie (WHO). Binnen deze brede benadering van gezondheid speelt naast de individuele gezondheidszorg een belangrijke rol, maar ook collectief gerichte publieke sector inclusief de overheid.

In hoofdstuk 3 wordt het gezondheidssysteem in de Verenigde Staten besproken aangezien het Amerikaanse systeem een voorloper is wat betreft gepersonaliseerde geneeskunde/ gepersonaliseerde gezondheidszorg. Van het Amerikaanse systeem kunnen we leren wat de stimulerende en belemmerende factoren zijn met betrekking tot een succesvolle integratie van gepersonaliseerde zorg in Europa te komen. In het Amerikaanse systeem staan 'keuzevrijheid' resp. "marktwerking" in de zorg beleidsmatig meer op de voorgrond dan "het principe van rechtvaardigheid en billijkheid". Dit kan leiden tot tekortkomingen in de gezondheidszorg waar het gaat om doelmatigheid, doeltreffendheid en rechtvaardigheid. Op basis van een nadere analyse van de mogelijke onderliggende oorzaken van deze tekortkomingen in, worden voorstellen geformuleerd om efficiëntie en effectiviteit te bevorderen en de onbillijkheid te reduceren in relatie tot de integratie van een gepersonaliseerd zorgmodel in Europa.

Zoals eerder vermeld, beschrijft deel II van het proefschrift de inhoudelijke aspecten van gepersonaliseerde geneeskunde/ gepersonaliseerde gezondheidszorg en wordt ook een specifiek praktijk model, Gentest, nader beschreven. Hoofdstuk 4 presenteert een toekomstvisie over gepersonaliseerde geneeskunde/ gepersonaliseerde gezondheidszorg, vanuit een praktijkgestuurd "bottom-up" perspectief, als tegenhanger van "top-down" processen. Op basis van literatuuronderzoek wordt dit 'praktijkgestuurd perspectief' nader beschouwd en wordt er gekeken naar de uitvoeringspraktijken van gepersonaliseerde geneeskunde/ gepersonaliseerde gezondheidszorg. Gepersonaliseerde geneeskunde/ gepersonaliseerde zorg kan immers voor verschillende doeleinden worden ingezet: om mensen zelf meer controle te geven over hun gezondheid, om de gezondheidszorg beter en efficiënter te maken en om iedereen gelijke toegang tot de gezondheidszorg aan te kunnen bieden. Bovendien toont dit hoofdstuk aan de hand van drie voorbeelden (Amerika, Frankrijk en Taiwan) aan dat niet alleen de visie van de overheid (op gepersonaliseerde geneeskunde/ gepersonaliseerde gezondheidszorg) een rol speelt bij de integratie, maar ook de verschillen in gezondheidssystemen en wetgeving in de verschillende landen. 
Waar in hoofdstuk 4 de verschillende facetten worden besproken voor de mogelijkheden tot persoonlijke geneeskunde, wordt in hoofdstuk $\mathbf{5}$ gepersonaliseerde zorg nader gedefinieerd in relatie tot begrippen als gepersonaliseerde geneeskunde, individuele geneeskunde. Aanvankelijk werd gepersonaliseerde geneeskunde synoniem gebruikt voor 'genomic medicine' (geneeskunde die zich enkel richt op de genetische (genoom) eigenschappen van een patiënt). Het is echter niet alleen ons genoom (DNA) dat ons uniek maakt en een rol speelt bij onze gezondheid. Factoren zoals fysieke beweging, voeding, leefstijl en blootstelling aan omgevingsfactoren (zoals rook of andere schadelijke stoffen) hebben immers eveneens een grote invloed op onze gezondheid. Een integrale benadering van de diversiteit aan gezondheidsdeterminanten wordt geplaatst in het perspectief van een paradigmaverschuiving naar een gepersonaliseerde zorg.

In hoofdstuk 6 wordt een concreet voorbeeld gegeven van toepassing van gepersonaliseerde zorg in de praktijk: Gentest. Gentest is een uitgebreid model dat rekening houdt met verschillende factoren en zo een risicotaxatie geeft met betrekking tot de kans om de meest voorkomende chronische, complexe ziekten te ontwikkelen. Om deze risicotaxatie te geven, maakt Gentest gebruik van persoonlijke gezondheidsinformatie, gedetailleerde leefstijl analyse, lichaamssamenstelling, genotype (DNA) en biomarkers die voorspellend zijn. Het model is oorspronkelijk ontwikkeld door het GENAR instituut in Ankara, Turkije. In Turkije is dit model al toegepast bij 500 personen. De positieve en negatieve punten die hierbij geleerd zijn, worden ook kort besproken in dit hoofdstuk.

In deel III wordt uitgezocht hoe een dergelijk model nu geïntegreerd kan worden in het Europees gezondheidssysteem.

Als we Gentest willen integreren in de eerstelijns gezondheidszorg in Europa, waar moeten we dan rekening mee houden? Hoe bepalen we de factoren waar we rekening mee moeten houden? Welke onderdelen van de gezondheidszorg en van het specifieke land moeten we onderzoeken? Hoofdstuk 7 richt zich op het beantwoorden van deze vragen. Om alle factoren te bepalen die een rol spelen bij de integratie, werd het 'Health Globe'kader uit Hoofdstuk 2 gebruikt. Zo werden 24 deelgebieden onderscheiden en vervolgens geordend om te identificeren welk van die deelgebieden cruciaal zijn voor een succesvolle integratie van een model als Gentest, met voorbeelden uit diverse Europese landen. M eer algemeen omvat Hoofdstuk 7 analyse van de belangrijkste factoren die hierbij een rol spelen

Hoofdstuk 8 beschouwt de interactie tussen de verschillende onderdelen van de gezondheidszorg en toepassingen van gepersonaliseerde zorg. Zoals eerder besproken is het de visie 'Health for All' van de Wereldgezondheidsorganisatie (WHO) die in 1977 het belang benadrukte van een bio-psycho-sociaal-ecologisch denkkader, ook op individueel niveau. Vanwege de interindividuele verschillen is het belangrijk om gepersonaliseerde geneeskunde / gepersonaliseerde zorg aan te kunnen bieden. Gentest is een model dat hieraan 
bijdraagt en wellicht kan zorgen voor 'Gezondheid voor iedereen'. Zulke modellen zullen echter alleen efficiënt blijven als de gedachtegang van de gezondheidszorg en beleidmakers proactief blijft en zich niet alleen op behandelen richt, maar ook op (vormen van) preventie van ziekte.

Naast de verandering in gedachtegang, wordt er in Hoofdstuk 8 ook een praktisch voorbeeld besproken hoe Gentest er in de praktijk uit kan zien.

Ten slotte worden in hoofdstuk 8 de belangrijkste conclusies van dit proefschrift weergegeven. Kort samengevat, dit proefschrift toont dat gepersonaliseerde geneeskunde / gepersonaliseerde gezondheidszorg een belangrijke rol kan spelen om chronische, complexe ziekten aan te pakken. Hiervoor is er wel een paradigma shift nodig en moet er meer nadruk komen te liggen op preventie. Om deze gedachtegang te bewerkstelligen en toch het 'Gezondheid voor iedereen'-principe te behouden, is het wenselijk dat innovatieve ontwikkelingen op het gebied van gepersonaliseerde zorg, gezondheidszorg en gezondheidsbeleid samengaan en op elkaar worden afgestemd. Voor een succesvolle integratie van een gepersonaliseerd zorg model zoals Gentest, moet de onderlinge relatie tussen de verschillende domeinen in acht worden genomen en als één geheel worden beschouwd. Hierin kan een kader zoals de 'Health Globe' ondersteuning bieden. Effectieve communicatie tussen de gebieden van de gepersonaliseerde geneeskunde, de gezondheidszorg en de beleidsmakers, kan het integratieproces wellicht verbeteren en versnellen. 


\section{Özet}

Kronik kompleks hastalıklar sağlık sistemleri üzerinde muazzam bir toplumsal ve finansal yük oluşturmaktadır. Bilimsel ve teknolojik gelişmeler sonucunda ortaya çıkan kişiye özel tıp ve kişiye özel sağlık hizmetleri kronik hastalıkların önlenmesi konusunda önemli fırsatlar sunmaktadır. Ancak 'kişiye özel' uygulamaların sağlık hizmetlerinin bir parçası haline getirilmesi, yani sağlık sistemlerine entegrasyonu önünde ciddi engeller bulunmaktadır.

Bu tezde kişiye özel sağlık uygulamalarının sağlık hizmetlerine entegrasyonu konusu ele alınmıştır. Konuyu etkili bir şekilde inceleyebilmek için kişiye özel koruyucu bir sağlık uygulaması olan 'Gentest' tezin vaka örneği olarak kullanılmıştır. Gentest gibi uygulamaya geçmiş, gerçek hayattan bir örneğin kullanılması, tez kapsamında incelenen konunun farklı boyutlarını ortaya koyabilir ve diğer kişiye özel uygulamalar için de dersler çıkarmayı sağlayabilir. Dolayısıyla bu tezin amacı, Gentest gibi kronik hastalıklardan korumaya yönelik bir kişiye özel sağlık uygulamasının Avrupa sağlık sistemlerinde birinci basamak hizmetlere nasıl entegre edilebileceğini incelemektir. Cevaba ulaşabilmek için tez kapsamında üç alt soru sorulmuştur: 1. Nereye entegre edilecektir? 2. Ne entegre edilecektir? 3. Entegrasyon nasıl gerçekleşebilir?

Tez, Gentest'i bir araç olarak kullanarak kişiye özel tıp, kişiye özel sağlık, ve sağlık sistemleri ve politikaları alanlarında bir seyahate çıkmıştır. I. Kısım entegrasyonun 'nerede' gerçekleşeceği sorusunu cevaplamış ve konumu tanımlamıştır: sağıık sistemleri. Bu bölümde sağlık sistemleri daha geniş bağlamı olan halk sağlığı kapsamında ele alınmıştır. II. Kısım 'ne' sorusunu cevaplamış ve öncelikle kişiye özel tıp ve sağlık alanını tanımlamıştır. Ardından, Avrupa'da sağlık sistemlerine entegre edilmesi düşünülen bir kişiye özel sağıı uygulama modeli olan Gentest'i tanıtmıştır. III. Kısım Gentest'in Avrupa'da birinci basmak sağlık hizmetlerine 'nasıl' entegre edilebileceği konusunda sistematik bir yaklaşım sunmuştur. Bu şekilde, söz konusu uygulama modelinin entegrasyonu sürecinde ele alınması gereken alanların ve konuların kapsamlı bir çerçevesini belirlemiştir. Bu üç kısımda yer alan bölümlerin özeti aşağıda sunulmuştur.

Bölüm 1'de kronik kompleks hastalıkların dünya çapında nasıl bir 'salgına' dönüştüğünü aktararak başlamıştır. Bu hastalıkların yükünün azaltılması için acilen çözümler geliştirilmesi gereklidir. Bunun için sağlık sistemlerinde bir paradigma değişimine gidilmesi gereklidir: daha proaktif, hastalıklardan ziyade yerine sağlığa ve sağlığı korumaya odaklanan, aynı zamanda kronik tedavi ve bakım hizmetleri veren ve sadece tıbbi hizmetleri değil, sağlık hizmetlerinin bütününü ele alan bir sisteme ihtiyaç vardır. Kişiye özel sağlık hizmetleri sağlık sistemlerinin bu yöndeki dönüşümünü desteklerken kronik hastalıklardan korunma açısından önemli fırsatlar sunmaktadır. Bu tezde kullanılan vaka örneği hastalık yükünün büyük kısmından sorumlu kronik hastalıklardan ve risk faktörlerinden korunma konusunda kapsamlı bir yaklaşım sunan ve kişiye özel bir sağlık 
uygulama modeli olan Gentest'tir. Bu model kronik hastalıkların oluşması, gelişmesi ve ilerlemesinin kişiye özel bir şekilde önlemeyi hedeflemektedir. Türkiye'deki pilot uygulamanın ardından Gentest Avrupa'da 'en iyi uygulama modeli' olarak belirlenmiştir. Bu noktada, Gentest gibi bir koruyucu sağlık uygulamasının esas uygulama alanının Avrupa'daki aile hekimliği/ birinci basamak sağlık hizmetleri, olduğu görülmüştür. Ancak, kişiye özel koruyucu sağlık modellerinin geniş toplum kesimlerine uygulanması konusunda başarılı örnekler henüz bulunmamaktadır. Bu nedenle, Gentest'in Avrupa'da birinci basamak sağlık hizmetlerine nasıl entegre edilebileceğinin incelenmesi gereklidir. Bunun için de yukarda sunulan 'nerde', 'ne' ve 'nasıl' sorularının cevapları aranmalıdır.

I. Kısım uygulama modelinin entegre edileceği konumu tanımlamayı amaçlamıştır. Öncelikle, bu tartışmanın bütünü ile 'halk sağlığı' (public health) ve 'sağlık sistemleri' kavramları üzerine inşa edilmesi gerektiği görülmüştür. Ancak bu kavramlar ve bunların birbiri ile ilişkileri konusunda netlik bulunmamaktadır. Bu nedenle, Bölüm $\mathbf{2}$ 'halk sağlığı' le ilişkili kavramları ve birbiri ile ilişkilerini tutarı bir şekilde açıklayan bir kavramsal çerçeve sunmuştur. 'Health Globe', yani 'Sağlık Küresi' adı verilen bu çerçevede sağlık sistemi ve bileşenleri de içerilmiştir. Bu girişimin amacı, üst düzey sağlık yöneticileri (politika üreticiler) ve onlara uluslararası platformlarda rehberlik edenler için tutarlı bir terminoloji sunmaktır. Dünya Sağlık Örgütü'nün 1977'de başlattığı 'Herkes için Sağlık' (Health for All) vizyonu 'Sağıık Küresi'nin kapsayıcı kavramsal çerçevesini oluşturmaktadır. 'Herkes için Sağlık' şiarı tarafından sağlığa getirilen geniş yaklaşım 'halk sağlığı' olarak tanımlanmıştır. Böylece, bireylerin sağlığını doğrudan ya da dolaylı bir şekilde etkileyen her türlü faaliyet 'halk sağlığı'nın kapsamına dahil olmaktadır. Bu, tıbbi ve tıbbi olmayan tüm hizmetler, devletlerin/hükümetlerin kararları ve uygulamaları ve kamu, özel ve sivil toplum sektörlerinin faaliyetlerini kapsamaktadır. Sağlık sistemi, 'Sağlık Küresi'nin bir parçasıdır ve sağlık bakanlığının sorumluluk alanına giren tüm faaliyetleri kapsar. Bu bölümde sağlık sisteminin amaçları ve işlevleri konusunda bir çerçeve de sunulmuştur. Gentest'in entegre edileceği bağlam birinci basamak sağlık hizmetleri olduğu için sağlık hizmetleri tanımlanırken birinci basamağın yeri ve kapsamı net bir şekilde gösterilmiştir.

Amerika Birleşik Devletleri ( $A B D$ ) kişiye özel tıp ve kişiye özel sağlık kavramları ve uygulamaları konusunda dünyanın önde gelen ülkelerinden biridir. Bu konudaki örneklerin ve yayınların büyük kısmı ABD kökenlidir. Bölüm 3'te, 'kişiye özel' uygulamaların ortaya çıktığı bağlamı anlamak ve Avrupa'da entegrasyonun nasıl yapılması ve yapılmaması gerektiği konusunda dersler çıkartmak için ABD sağlık sistemi incelenmiştir. Bu bölümde $A B D$ 'nin tutarlı bir şekilde tasarlanmış bir sağlık 'sistemi'ne sahip olmadığını görülmüştür. Politika (policy) değerleri açısından ABD 'özgürlük' ile 'serbest piyasa'yı 'hakkaniyet' değerinin üzerinde tutmuştur. Bu durum sağlık sisteminin temel sonuçları olan etkililik, verimlilik ve hakkaniyet açısından ciddi sorunlara neden olmuştur. Bu bölümde temel sorunlar ve altında yatan nedenler ortaya konduktan sonra Amerika'da sağlıkta etkililik ve verimliliği artırmak ve eşitsizlikleri azaltmak için nasıl bir sağık politikası ve sağlık sistemi 
geliştirilmesi gerektiği konusunda bir çerçeve sunulmuştur. Verilen öneriler ağırlıklı olarak Avrupa ülkelerinin tecrübelerine dayanmıştır.

II. Kısım hem kişiye özel tıp ve kişiye özel sağlık hizmetleri bağlamını hem de vaka örneğimiz olan Gentest'i tanımlayarak neyin entegre edileceğini ortaya koymayı amaçlamıştır. İlk olarak, Bölüm 4'te tıpta ve sağlıkta görülen 'kişiye özelleşme' vizyonunun farklı kişi ve kurumlar tarafından farklı şekillerde tanımlandığını aktarmıştır. Bu konuda standart tek bir tanım bulunmamaktadır. Ayrıca, tanımlamalar alanında sözü geçen kişiler ve komiteler tarafından yapılmıştır. Dolayısıyla, gerçek hayatta, yani sağlık hizmetleri sahasında ne olup bittiğini yeterince yansıtmıyor olabilir. Bu tartışmaya farklı bir açıdan yaklaşmak için Bölüm 4'te 'uygulamaya dayalı bakış acısı' (ya da 'tabandan yaklaşım') kullanılmıştır. Bilimsel literatürün sistematik bir şekilde taranması sonucunda belirlenmiştir yayımlanmış 'kişiye özel' uygulamalar belirlenmiş, daha sonra da bunların içeriği analiz edilmiştir. Sonuç olarak 'kişiye özel' uygulamaların çok çeşitli olduğu ve 'kişiye özel' kavramının tanımında ciddi farklılıklar bulunduğu görülmüştür. Uygulama şekli ve bağlamı açısından bakıldığında, 'kişiye özel' bir uygulama sağlık pazarında bir meta ya da sağlık hizmetlerinde bir sunum şekli olabileceği gibi yeni geliştirilen teknolojik uygulamalarla ilgili bir anahtar kelime olarak de kullanılabilmektedir. 'Kişiye özel' uygulamalar çok çeşitli gruplara farklı amaçlar için hizmet edebilir. Bu geniş yelpazede bireyleri hedefleyen uygulamalar (sağlıklarını bireysel olarak kontrol etmeye başlamaları için), sağlık kurumlarının uygulamaları (artan rekabet ortamında sağlık hizmetlerinde etkililik ve verimliliklerini artırabilmeleri için) veya ulusal sağlık sistemlerinin programları (etkililik ve verimliliğin yanı sıra hakkaniyeti de içerecek şekilde performanslarını artırabilmeleri için) yer almaktadır. Ayrıca, üç ülke örneği incelendiğinde (ABD, Fransa ve Tayvan), uygulamaların sağlık hizmetlerine başarılı bir şekilde entegre edilebilmesi için sağlıkla ilgili yeniliklere (innovasyon) karşı ülkedeki tutumun yanı sıra sağlık sistemi ve politikaları bağlamının da kritik önem taşıdığı görülmüştür.

Bölüm 4'te mevcutta yer alan kişiye özel uygulamaların çeşitli boyutları ortaya konduktan sonra Bölüm 5'te bu tez kapsamında 'kişiye özel sağlık hizmetleri'nin ne olduğu tanımlanmıştır. Bu alanda sıkça kullanılan 'kişiye özel tıp', 'kişiye özel sağlık', 'bireysel tıp' gibi kavramların bu makalenin (bölümün) yazarları için ne ifade ettiği ortaya konmuştur. Kavramların gelişmeye başladığı ilk dönemlerde 'kişiye özel tıp' ve 'genomik tıp' kavramları eş kabul edilmiştir. Ancak genom bireyleri özel ve biricik yapan tek faktör değildir. Genom, patojenler ve toksinlere maruziyet, sosyoekonomik faktörler ve fiziksel aktivite, beslenme ve diğer yaşam tarzı faktörleri sürekli olarak birbiri ile etkileşmektedir. Bireyler bunları kapsayacak şekilde tüm bağlamları içinde ele alınmalıdır. Bu nedenle hem bilimsel çalışmalar, hem de kişiye özel sağlık hizmetleri bağlamında sağlığın çeşitli belirleyicilerini kapsayan, bütünleştirici bir yaklaşım uygulanması gereklidir. Bu bölümün sonucunda sağlık sistemleri ve politikalarının kişiye özel yaklaşımlara yönelik dönüşüm için hazırlanması gerektiği de vurgulanmıştır. 
Bölüm 4 ve 5 'te kişiye özel tıp ve kişiye özel sağlık uygulamaları zemini ortaya konduktan sonra Bölüm 6'da bu tez kapsamında kullanılan kişiye özel tıp uygulaması tanıtılmıştır: Gentest. Gentest sık görülen kronik hastalıkların önlenmesini hedefleyen kapsamlı ve bütüncül bir kişiye özel sağlık hizmeti modelidir. Bireylerin kişisel sağlık bilgilerini, ayrıntılı yaşam tarzı analizlerini, vücut bileşimlerini, genotiplerini ve diğer biyomarkerlarını kapsayan bu uygulama sık görülen kronik hastalıkların ortaya çıkması veya ilerlemesini kişiye özel bir şekilde engellemeyi hedeflemektedir. Gentest'in misyonu bireylerde davranış değişikliğini sağlamak ve bireylerin sağığını onların sağlık önceliklerine göre yönetmektir. GENAR Toplum Sağlığı ve Genombilim Araştırmaları Enstitüsü tarafından Ankara'da geliştirilmiştir. Bu bölümde Gentest'in yetkili uygulayıcılar tarafından 500'ün üzerinde bireye hangi modelle uygulandığı aktarılmıştır. Yetkili uygulayıcı olmak için sağlık profesyonelleri (ağırlıklı olarak hekimler) özel bir eğitim programı tamamlamışlardır. Bu bölümde ayrıca Gentest'e yönelik olumlu ve olumsuz eleştirilere de kısaca yer verilmiştir.

I. Kısım konumu, yani sağlık sistemlerini, II. Kısım ise kişiye özel koruyucu uygulamayı (Gentest) 'kişiye özel sağlık' bağlamında tanımlamıştır. Bunlara dayanarak III. Kısım, bu uygulama modelinin (Gentest) konuma, yani sağlık sistemlerine nasıl entegre edilebileceğini incelemiştir.

Eğer Gentest gibi bir uygulama modelini Avrupa'da birinci basamak sağlık hizmetlerine entegre etmek istiyorsak hangi konuları incelememiz gerekir? Bunları sistematik ve analitik bir şekilde nasıl belirleyebiliriz? Bir ülkenin sağıı sisteminde hangi alanların incelenmesi gerekir? Bölüm $\mathbf{7}$ bu soruları cevaplamayı amaçlamıştır. Entegrasyonla ilgili tüm konuların belirlenebilmesi için 2. Bölüm'de sunulmuş olan 'sağlık sistemleri çerçevesi'ne dayanarak bu bölümde sistematik bir yaklaşım geliştirilmiştir. Bu kapsamda, sağlık sisteminin işlevleri bu sistematik yaklaşımın kavramsal çerçevesini oluşturmuştur. Bölüm 4'te aktarıldığı üzere 'kişiye özel' uygulamalar çok çeşitli olup tek genel bir hizmet sınıfı altında toplanması mümkün değildir. O nedenle bu bölümde Gentest'in Avrupa'da birinci basamak sağlık hizmetlerine entegrasyonu örneği kullanılmıştır. Sonuç olarak sağlık sisteminin işlevleri altında 24 alan belirlenmiştir. Bu sistematik yaklaşım başta Hollanda ve İngiltere olmak üzere çeşitli Avrupa ülkelerinden örneklerle zenginleştirilmiştir. Daha sonra, bu 24 alandan hangilerinin entegrasyon için 'kritik' ve hangilerinin 'yüksek öncelikte' olduğu belirlenmiştir. Özetle Bölüm 7, bir uygulama modelinin Avrupa sağlık sistemlerinde birinci basamak hizmetlere entegrasyonu ile ilgili konuların belirlenmesi ve değerlendirilmesi için sistematik bir yaklaşım ortaya koymuştur. Bu yöntem farklı uygulama modelleri ve ülkelere uygulanabileceğinden kişiye özel tıp ve kişiye özel sağlık uygulamalarının sağlık sistemlerine entegrasyonu sorununa kapsamlı bir çözüm önerisi getirmektedir.

Bölüm 8 konuya daha yukardan bakarak sağlık sistemleri ve kişiye özel sağlık uygulamaları bağlamları arasındaki etkileşimle ilgili bazı görüşler ortaya koymuştur. 1977'de ortaya konan 'Herkes için Sağlık' vizyonu bireylerin biyo-psiko-sosyo-ekolojik bağlamını 
anlamanın önemini vurgulayan 'ekolojik' yaklaşımın gelişmesini sağlamıştır. Her bireyin bağlamı diğerlerinden az ya da çok farklıdır. Bireysel bağlamı anlayıp buna göre müdahaleler geliştirmek sağlığa kapsamlı bir şekilde yaklaşma konusunda önemli fırsatlar sunmaktadır. Gentest gibi bireylerin sağlığını, risklerini ve hastalıklarını kapsamlı bir şekilde ele alan bütüncül ve kişiye özel sağlık uygulamaları ile 'Herkes için Sağlık' hedefine yönelik önemli mesafeler kat edilebilir. Ancak bu tür uygulamaları bünyesine başarılı bir şekilde dahil edebilmesi ve 'Herkes için Sağlık' hedefine ulaşabilmesi için sağlık sistemlerinin daha proaktif, hastalıklardan ziyade yerine sağlığa ve sağlığı korumaya odaklanan, aynı zamanda kronik tedavi ve bakım hizmetleri veren ve sadece tıbbi hizmetleri değil, sağlık hizmetlerinin bütününü ele alan bir sisteme evrilmesine, bunun için de yenilikler yapılmasına ihtiyaç vardır. Bunun için 'topluma dayalı koruyucu hizmetler mi, yüksek riskli bireylere yönelik koruyucu hizmetler mi?' gibi bazı geleneksel kavramların da yeniden gözden geçirilmesi gereklidir. Ayrıca, kronik hastalıkların gelişimindeki süreklilik göz önüne alındığında koruyucu müdahaleler için yeni bir sınıflama olarak 'kişisel sağlık yönetimi', 'kişisel risk yönetimi' ve 'kişisel hastalık yönetimi' kavramları gündeme girebilir. Bu tür koruyucu kişiye özel sağlık uygulamalarının topluma proaktif bir şekilde sunulabilmesi için birinci basamak hizmetlerinde yeniliklere ve güçlendirmeye ihtiyaç vardır. Gentest gibi kişiye özel sağık uygulamaları sağlık sistemlerindeki paradigma değişimini gerçekleştirmek için pratik bir fırsat olarak da kullanılabilir.

Sağlık sistemlerindeki yenilikler tek başına yeterli değildir; kişiye özel sağlık hizmetlerinde de sürekli yenilenmesi gerekir. Öncelikle, 'kişiye özel' uygulamaların kapsamlı bir nitelik kazanması için yeniliğe ihtiyaç vardır. Bir laboratuvar analizi gibi tek bir testi kullanan, tek bir hastalığın tanısı veya tedavisi ile ilgili bir uygulama 'kişiye özel' olabilir mi? İster yüksek ister düşük teknolojik olsun, ister biyolojik, ister biyolojik olmayan bir faktör olsun, tek bir faktörü ele alan bir uygulama ne kadar 'kişiye özel' olabilir? Bir uygulamanın 'kişiye özel' olması için bireysel bağlamın bütüncül bir şekilde anlaşılması ve kullanılması gereklidir. Ayrıca, Gentest gibi kişiye özel sağlık uygulamalarının hedeflenen ülkelere ve sağlık sistemlerine uygun hale gelmesi için sürekli olarak yenilenmesi gerekir. Kişiye özel tıp ve kişiye özel sağlık alanında çalışanların şunun farkına varmaları gerekir: gerçekleştirdikleri bilimsel keşifler ile geliştirdikleri teknolojiler ve araçların toplumda belirgin bir etki yapabilmesi için bu uygulamaların sağlık sistemlerine entegre olması konusunda kapsamlı bir yaklaşım gerekir.

Tezin metoduyla ilgili olarak Bölüm 8'de yapılan tartışmada gerçek hayatta kullanılan somut bir uygulamanın bu tezde ele alınmasının avantajları ve bu alandaki araştırmalara nasıl bir katkıda bulunabileceği aktarılmıştır.

Bölüm 8 bu tezin temel sonuçları ile bitirilmiştir. Özetle, kişiye özel sağlık uygulamaları hızla artan kronik kompleks hastalık yükü ile mücadele için önemli fırsatlar sunmaktadır. Aynı zamanda, sağlık sistemlerinde ihtiyaç duyulan paradigma değişimine de hizmet etmektedir. Bu değişimi gerçekleştirmek ve 'Herkes için Sağlık' amacına ulaşabilmek için 
kişiye özel tıp ve kişiye özel sağlık alanındaki yenilikler ile sağlık sistemleri ve politikaları alanındaki yenilikler birlikte evrilmeli, bu iki alan etkileşim içinde olmalıdır. Bu şekilde yeniliklerin birbirlerinin ihtiyaçlarına göre şekillenmesi mümkün olabilir. Kişiye özel sağlık uygulamalarının başarılı bir şekilde hizmete geçmesi için entegrasyon ile ilgili konular parça parça değil, sistematik bir şekilde ele alınmalıdır. Sağlık sistemi kavramsal çerçevesi, entegrasyon ile ilgili konuların belirlenmesi ve değerlendirilmesi için gereken analitik çerçeveyi sunmaktadır. Kişiye özel tıp ve kişiye özel sağlık alanı ile sağlık sistemleri ve politikaları alanı arasında etkili bir diyalog kurulması entegrasyon sürecinin daha etkili ve hızlı olmasına yardımcı olacaktır. 
Valorization 
The dissertation started with the following paragraph:

"Every day, we read about new scientific discoveries and technological advancements in the newspapers. The news are very promising for medicine and health, in particular in reference to 'genetics' in the past 15-20 years: "Diabetes gene is found!", "Heart attack gene is found!", "Cancer gene is found", "We'll be able to prevent diabetes in 10 years!"... However, we don't see these 'genes' in health services. Somehow, they don't complete their journey from the laboratory to the society"

This journey is actually a winding road, as it involves in science, technology, health and society domains and various hurdles in each. The innovations are usually envisaged to be transferred from one domain to the other in this order. However, as presented in Chapter 4 , innovation does not follow a simplified linear process or a 'pipeline', which ends when the 'product' is out in the market. Innovations co-evolve with the involvement of different domains.

The health domain, which includes the health system, plays a crucial role in effective integration of innovations. Therefore, this PhD research focused on this domain, and tried to answer the question how a personalized health care model can be integrated into health systems in Europe. While answering it, a real life practice model, i.e. Gentest, was used as the case. Thus, the topic of this dissertation was involved with developing guidance for valorization of scientific and technological developments in the field of personalized medicine and personalized health care.

Use of a case to investigate integration issues provided a novel approach that hasn't been applied in this field before. As the starting point was a real life example that was seeking for integration into new health systems, the research was highly practice oriented rather than being solely a theoretical one. Thus, the 'valorization' has already been an inherent priority of this research.

The implications of the dissertation are two folds. On one hand, the dissertation may facilitate the fields of personalized medicine and health care and health systems to learn to talk to each other and work together. On the other hand, the dissertation provides a useful tool, i.e. the systematic approach presented in Chapter 7, to guide integration of personalized practices into different health systems.

\section{Implications for the fields of personalized medicine and health care and health systems}

As the topic of the dissertation was involved with valorization of personalized practices, several implications have been presented in Chapters 7 and 8 . Here, these will not be 
repeated, but concrete messages for the fields of personalized medicine and health care and health systems will be put forward.

Health systems are facing a huge problem with the rapidly increasing burden of chronic complex diseases. They need to shift towards proactive and health oriented services focused on prevention as well as chronic cure and care, covering the whole spectrum of health care (not only medicine). Rather than running separate programs for each lifestyle/ disease area, holistic approaches to disease prevention and health promotion should be developed. Personalized health care practices can be the answer of health services to these needs. At this point, the practice model used in this dissertation, Gentest, can inspire integrative and holistic programmes not only for prevention of late onset chronic diseases, but also in other fields such as child and youth health care, with a life course perspective.

To achieve integrative and holistic approaches to health, innovations in the fields of personalized medicine and health care, and health systems need to co-evolve. To do that, people working in these fields should first start talking to each other, learning one another's language, and developing ways to collaborate. This doesn't simply mean making conferences or meetings that bring people from these fields together, and provide 'networking' opportunities. Moreover, many of the so-called 'collaborative' projects that bring different fields together do not always help them collaborate. Most of such projects assign specific tasks or work packages to groups that are from the same discipline or field. Thus, each discipline does its own work; they don't necessarily work together in a crossdisciplinary way.

To facilitate co-evolution, a coherent and concrete agenda should be provided to people in the fields of personalized medicine and health care, and health systems. They should first start working on smaller and simpler projects, where they can start learning each other's languages and priorities. Then, they can move towards bigger and more complex projects.

These fields should also be made aware of the differences in their perspectives and jargons. As provided in Chapter 4 with a bottom-up approach, personalization may mean different things for the practices. Therefore, special effort should be paid towards harmonization of the language. As provided in Chapter 8, personalization cannot simply mean use of molecular technologies, or any technology. The harmonization of the language should include clarification of the purpose and means of personalization. 


\section{Implications for personalized practices which will be integrated into different health systems}

The $\mathrm{PhD}$ research has implications for personalized practices in general, as well as the specific practice model that was used in this dissertation: Gentest.

In general, the science and technology (including academia and industry) domains seem to have a quest for using the knowledge and tools they are developing to personalize medicine and health care. These domains take leadership roles as thought leaders or entrepreneurs. To successfully integrate the knowledge and tools they generate, they first need to understand that 'personalization' is not only about genetics, or technology. It involves 'practices', as well as health systems that surround them. At this point, the dissertation provides a valuable perspective to them to understand what is relevant in health systems.

For personalized practices which seek for integration into health systems, the dissertation provides a systematic approach (Chapter 7) which can be applied to different cases. It can be used not only for 'personalized medicine and health care', but also in general for innovations in health. The author of the dissertation is currently presenting this approach to various organizations involved in scientific and technological innovations in health. When it is picked up by others and applied on different cases, it may evolve into a practical tool for investigation of integration issues for innovations in health.

The dissertation also has several implications for the case of Gentest, as provided in Chapters 7 and 8 . For successful valorization of Gentest, it is important to find a country where the health system supports the crucial areas, as well as most of the priority areas, described in Chapter 7. Then, right partnerships should be developed within the academia, (local) health authorities, industry, and civil society, possibly leading to a public private partnership initiative. This partnership should agree on a roadmap which includes adaptation and further development of the model within the target country, software development, piloting, and evaluation phases.

Overall, this dissertation has large potential for valorization. It can be used to facilitate coevolution of health systems and personalized health care practices. The research also has profound implications on integration of personalized practices into health systems. The practice model used in the dissertation, i.e. Gentest, can inspire holistic and integrative programmes not only for prevention of chronic diseases, but also for other health fields such as child and youth health care with a life course perspective. 
ilim ilim bilmektir Ilim kendin bilmektir Sen kendini bilmezsen

Ya nice okumaktır

Okumaktan mana ne

Kişi Hakk'ı bilmektir

Çün okudun bilmezsin

Ha bir kuru emektir

Okudum bildim deme

Çok taat kıldım deme

Eri Hak bilmez isen

Abes yere yelmektir

Dört kitabın manası

Bellidir bir elifte

Sen elif dersin hoca

Manası ne demektir

Yunus Emre der hoca

Gerekse var bin hacca

Hepsinden iyice

Bir gönüle girmektir
Knowledge should mean a full grasp of the path to knowledge Knowledge means to know yourself, heart and soul.

If you have failed to understand yourself, Then all of your reading has missed its call.

What is the purpose of reading those books?

So that Man can know the God/ the Truth

If you have read, but failed to understand,

Then your efforts are just a barren soil.

Don't boast of reading, mastering science

Or of all your prayers and obeisance.

If you don't identify M an as God,

All your learning is of no use at all.

The true meaning of the four holy books

Is found in the alphabet's first letter.

You talk about that first letter, teacher;

What is the meaning of that - could you tell?

Yunus Emre says to you, teacher, Make the holy pilgrimage if need be A hundred times - but if you ask me, To win a heart is the best of all.

Yunus Emre ${ }^{1}$

\footnotetext{
${ }^{1}$ Yunus Emre was a Turkish poet and Sufi mystic who lived in the 13-14th centuries in Anatolia.
} 
Acknowledgements

Dankwoord | Teșekkürler 
"PhD" is an individual degree. Nevertheless, it cannot be achieved without people guiding and supporting the PhD candidate. Here, I would like to thank everybody who has supported me conducting the research and writing this dissertation.

First, and foremost, when doing a PhD, it is very important to have the right team around you. It took me some time to find the right one, but eventually, I worked with a fantastic team: Prof. Dr. Frans Feron as my supervisor, and Prof. Dr. Anja Krumeich and Dr. Elena Syurina as my co-supervisors. They were dedicated to provide all the support I needed to complete my PhD. Likewise, Dr. Serdar Savaş, who has been supporting many parts of my PhD work and process externally, provided very valuable input. I wholeheartedly appreciate the support and guidance they have provided me.

I would like to convey my gratitude to Professor Frans Feron for being the supervisor of my PhD. It was a great pleasure to work with someone who shares the passion for transformation of health and health services towards a more preventive, predictive, participatory and personalized approach. He supervised me with the balance of methodological scrutiny and practice orientation. As I worked more with Frans, I saw that he embraced the holistic and 'personalized' approach not only in health services, but also when supervising researchers who worked with him. With his 'human centered' approach, he changed my life drastically. I owe him a lot.

It has been a great experience to work with Professor Anja Krumeich as my co-supervisor. Working with her and learning from her introduced me new dimensions in health and health practices, as well as their interrelations with science, technology and society. With her holistic approach, she provided critical guidance in structuring this dissertation and completing it. I deeply thank her for all the guidance and contribution she made during the research and writing phases.

I enjoyed very much working closely with Doctor Elena Syurina as my co-supervisor. It was a pleasure to work with such a systematic thinker and a result oriented person. She has been very responsive to all my inquiries and provided full support during various phases of the publications. I deeply thank her for this great support, as well as the encouragement and motivation she gave in the moments I most needed.

I would like to thank the members of the assessment committee, Prof. Dr. Nanne de Vries, Prof. Dr. David Townend, Dr. Petra Verdonk and Dr. Heidi C. Howard for reading my dissertation and their presence during the PhD ceremony. It is a great honor to have experts of such caliber as the part of my assessment committee.

I am very grateful to Doctor Serdar Savaş for supporting my PhD as an external project team member. It was a privilege to learn from him what 'Health for All' means in theory and practice during the long years we have worked together. This eventually allowed me 
to orient my PhD work towards 'Health for All' vision. During the PhD, he provided valuable guidance on health systems and policies aspects of the dissertation with his deep experience in this field.

I would like to thank very much Prof. Dr. Albert Scherpbier and Prof. Dr. Nanne de Vries in their capacities as the Dean and Vice-Dean of the Faculty of Health, Medicine and Life Sciences, for placing me at the Department of Social M edicine.

I thank to the Department of Social Medicine very much for giving me a warm welcome and a peaceful environment for my research. It was a great experience to be a part of this department, which included Hans Bosma, Nicole Boumans, Karin Braeken, Elisabeth Dorant, Yvonne Goertz, Inge Houkes, Marleen Janssen, Gonnie Klabbers, Annemarie Koster, Isel van Noppen, Katerina Putnik, Cobie Martens, Angelique de Rijk, Bram Rooijackers, Audrey Simons, Pien Struijk, Marie-Jose Theunissen, Rineke Vasse, and all other colleagues. I will remember very well our annual days out, doing a variety of things as a team. In particular, I thank Danielle Groffen and Nicole Hoefsmit for the warm welcome they gave to me at our room in the office. I thank all my office mates for keeping my days lively: Anne, Anniek, Belle, Brenda, Dominique, Fiona, Karina (in the next door), Maren, Marit, Naomi, and Romy.

Gentest provided the real life example that was used in this PhD research. Therefore, I would like to convey my gratitude to all colleagues at GENAR Institute for Public Health and Genomics Research. It was a great pleasure to work with Dr. Sema Erge, Dr. Şefayet Karaca and other members of our multidisciplinary team, under the leadership of Dr. Serdar Savaş.

During my years at Maastricht University, I've met with valuable professionals. I'd like to expand my thanks to them as well, since they supported and encouraged me in different ways: Elena Ambrosino, Ivan Brankovic, Kristine Sorensen, Bahar Taneri, Erik Haakma and Willem Wolters. In particular, I am grateful to Petra Verdonk for her support at the difficult times.

Juggling professional and family responsibilities is not easy while doing a PhD. I sincerely thank our family in Turkey: my parents Çelik and Hülya Cesuroğlu, my brother Sungur Cesuroğlu, my grandfather Ali Cesuroğlu, my aunt Sevda Örten; our family in Utrecht: my parents-in-law Jan and Ton de Langen, my brothers- and sisters-in-law Robert de Langen, Miranda van Spelde, Michel de Langen and Marije Kolkman, and all Tantes and Ooms; our neighbor Mariella Naranjo; and all friends from near and far, in particular Marianne Linthorst, Emre Almac, Melike Yanev, Deniz Arik Binbay, Tolga Binbay, and Eren Kaya. Anneciğim, babacığım, kardeşim, dedeciğim ve teyzeciğim; tüm destekleriniz için sizlere çok teşekkür ederim! Ma en $\mathrm{Pa}$, Robert, Miranda, M ichel, Marije, onze tantes en ooms, en Mariella; heel erg bedankt voor jullie steun! 
Special thanks go to Ivan Brankovic and Emre Almac for accepting to be my paranymphs, as well as my friend Serenad Yilmaz for designing the cover of this book.

You must have heard of the cliché: "Every successful man has a woman behind". The correct version, in my opinion, should be: "Every successful person has a person next to him/her". Without Marco standing by me, I wouldn't have overcome all the obstacles and succeeded in this endeavor. Our children Yunus, M ina and Alanur gave me the strength to endure the long and winding road I've been through. I thank my husband and children from the bottom of my heart and dedicate this manuscript to them. 
Perform anonymous service. Whenever we do good for others anonymously, our sense of intrinsic worth and self-respect increases. ... Selfless service has always been one of the most powerful methods of influence.

Stephen Covey 
CV \& Publications 


\section{Curriculum Vitae}

Tomris Cesuroglu was born in 1978 in Ankara, Turkey and grew up in Mersin, which is a town on the south coast of Turkey. She graduated from içel Anatolian High School in 1996 and was admitted to Hacettepe University Faculty of M edicine in Ankara. During her studies, she carried out and/or led various volunteered projects on medical education and reproductive health at national (Turkish Medical Students' International Committee TurkM SIC) and international level (International Federation of M edical Students' Associations - IFMSA).

She obtained her doctor of medicine (MD) degree in 2002. The same year she started working in Istanbul at BSS - United Health Systems consultancy which operated on various areas including health systems and policies, Turkish health reforms, health services planning, reproductive health, continuous quality improvement in health care organizations and feasibility studies. At BSS, she worked on various projects on the above mentioned areas and that served to a large clientele including UN agencies, M inistry of Health, World Bank, health care providers, pharmaceutical sector, and others.

She worked in the establishment of GENAR Institute for Public Health and Genomics Research in Ankara (2004), which consisted of a high-throughput genotyping laboratory, a center for lifestyle and nutrigenetics research, and a center for personalized medicine and pharmacogenetics. She headed the last center and was GENAR's Research and Development Coordinator. She executed various scientific and technical activities related to genetics of complex diseases, pharmacogenomics, personalized medicine, personalized health care and public health genomics as a researcher or principle investigator. She made a major contribution to development of Gentest, which is an integrative and personalized health care model for prevention of complex diseases. She also coordinated GENAR's research and $R \& D$ projects funded by European Commission and national funds.

In 2010, she moved to Maastricht University since she was awarded with the Marie Curie Fellowship of the European Commission (Intra-European Fellowship) to carry out policy research on implementation of the Gentest model in health systems in Europe at the Institute for Public Health Genomics (2011-2014). In 2014, she started working as a researcher at the Department of Social Medicine, Maastricht University, and continued her research on integration of innovations into health systems.

She is one of the founding members of Turkish Society for Public Health Genomics and Personalized M edicine (TOGEN, 2005) and currently its vice president. She organized and participated numerous international scientific events in her area and gave various lectures at universities and national and international platforms. She has also been serving as an expert evaluator to European Commission for Framework Programme 7 and Horizon 2020.

All these activities allowed her to experience the full breadth of innovations in health care from research and development to business aspects, and from policy to health care prac- 
tice. She is currently exploring projects where she can use this multidisciplinary and intersectoral experience involving science, technology, health policy and practice, and society domains. This also involves application of the systematic approach presented in this book to different innovations for their integration into health systems.

As physician, researcher and mother of three children, Tomris Cesuroglu writes blog articles on breastfeeding and other child health and development issues in a popular and well-respected parenting blog in Turkish (Bebek Yapim Bakim Onarim). As a volunteered work for public interest, she has been supporting mothers on breastfeeding issues through social media extensively, as well as trying to develop systems to support them. She is involved with promotion of breastfeeding and advocacy for effective implementation of the International Code of Marketing of Breast-M ilk Substitutes in Turkey. She is a member of IBFAN - International Baby Food Action Network and a new civil society movement in Turkey called Virtuous Citizenry Initiative (Erdemli Yurttaş Hareketi).

She is married to M arco de Langen and they have three children: Yunus (6), M ina (6) and Alanur (3). 


\section{List of publications and presentations}

\section{Publications of the present dissertation \\ Chapter 2:}

Savas BS, Cesuroglu T. Health Globe: A Proposal for a Coherent Terminology of Public Health Concepts. Submitted.

\section{Chapter 3:}

Savas BS, Cesuroglu T. Health for America: European Arguments for a Paradigm Shift. In: Shah A, Colombano J, editors. Learning from the World: New Ideas to Redevelop America. Palgrave Macmillan; 2013. p. 169-86.

\section{Chapter 4:}

Cesuroglu T, Syurina E, Feron F, Krumeich A. The Other Side of the Coin for Personalized Medicine and Health Care: Content Analysis of 'Personalized' Practices in the Literature. Submitted.

\section{Chapter 5:}

Cesuroglu T, van Ommen B, Malats N, Sudbrak R, Lehrach H, Brand A. Public health perspective: from personalized medicine to personal health. Personalized Medicine. 2012;9(2):115-9.

\section{Chapter 6:}

Cesuroglu T, Karaca S, Erge S. A practice model for personalized healthcare with a public health genomics perspective. Personalized Medicine. 2009;6(5):567-77.

\section{Chapter 7:}

Cesuroglu T, Syurina E, Krumeich A, Feron F, Savaş S. A systematic approach to integration of a preventive personalized health care model into health systems. Submitted.

\section{Publications outside this dissertation}

Personalized medicine and health care, and genetics of complex diseases related publications

\section{Articles in scientificjournals}

Karaca S, Erge S, Cesuroglu T, Polimanti R. Nutritional habits, lifestyle, and genetic predisposition in cardiovascular and metabolic traits in Turkish population. Nutrition. Epub 2015 Dec 29.

Karaca S, Bozkurt NC, Cesuroglu T, Karaca M, Bozkurt M, Eskioglu E, et al. International warfarin genotype-guided dosing algorithms in the Turkish population and their 
preventive effects on major and life-threatening hemorrhagic events. Pharmacogenomics. 2015;16(10):1109-18.

Karaca S, Cesuroglu T, Karaca M, Erge S, Polimanti R. Genetic diversity of diseaseassociated loci in Turkish population. Journal of Human Genetics. 2015;4(60):193-8.

Karaca S, Karaca M, Cesuroglu T, Erge S, Polimanti R. GSTM 1, GSTP1, and GSTT1 genetic variability in Turkish and worldwide populations. American Journal of Human Biology. 2014;27(3):310-6.

\section{Abstracts}

Erge S, Karaca S, Aksoy N, Kankilic T, Cesuroglu T. "The Role of Functional Polymorphisms Involved in Homocystein Metabolism" in $22^{\text {nd }}$ International IFCC Congress, 22-26 Jun. 2012, Istanbul, Turkey. Clinical Chemistry and Laboratory Medicine (CCLM). 2014;52(Supplement):818.

Cesuroglu T, Savas S, Morré S, Brand A, editors. Implementation of Personalized Health Care in Health Systems. Genetica Retraite; 2012 15-16 M ar. 2012; Rolduc-Kerkrade: M aastricht University; 2012.

Kaymaz A, Karaca S, Cesuroglu T, Kankilic T, De Langen M. "Allelic Frequency of Genetic Variants Associated with Hypertension in Turkish Population" in $24^{\text {th }}$ National Biochemistry Congress, 25-28 Sep. 2012, Konya, Turkey. Turkish Journal of Biochemistry. 2012;37(S1):156.

Karaca S, Cesuroglu T, Kankilic T, Aksoy NH. "Allelic frequency of single nucleotide polymorphisms involved in cancer related bioprocesses" in $37^{\text {th }}$ International FEBS \& $22^{\text {nd }}$ IUBM B Congress, 4-9 Sep. 2012, Sevilla, Spain. FEBS J. 2012;279:322.

Cesuroglu T, Brand A. "Implementation of Personalized Healthcare in Health Services in the Context of Public Health Genomics-Looking for the Broader Picture" in EPM AWorld Congress 2011 in Bonn, Germany. The EPMA journal. 2011;2(Suppl 1):79-80.

Karaca S, CesurogluT, Kocaman E. "M ALDI-TOF MS Based Genotyping of Single-Nucleotide Variations Predisposing to Cardiovascular Diseases" in IX. National Medical Genetics Congress 01-05 Dec. 2010, Istanbul, Turkey. Clin Genet. 2010;78(s1):57. doi: 10.1111/j.1399-0004.2010.01559.x.

Karaca S, Cesuroglu T, Kaymaz A. "Allelic Frequencies of Genetic Variants Associated with Bone Mineral Density, in Turkish Population" in IX. National Medical Genetics Congress, 01-05 Dec. 2010, Istanbul, Turkey. Clin Genet. 2010;78(s1):42.

Cesuroglu T, Karaca S, De Langen M. "Frequency of some genetic variation, known as risk factors for insulin resistance, type II diabetes and obesity, in Turkey" in $22^{\text {nd }}$ National 
Biochemistry Congress, 27-30 Oct. 2010, Eskisehir, Turkey. Turkish Journal of Biochemistry. 2010;35(S1):172.

Cesuroglu T. "Chronic Complex Diseases and Genetics" in Turkish Society of Molecular Medicine, $3^{\text {rd }}$ International Congress of Molecular Medicine, May 5-8, 2009, Istanbul, Turkey. IUBM B Life. 2009;61(3):303-.

Cesuroğlu T. "The Relationship Between Nutrition, Disease \& Aging: A Review" in the Congress of the Association of Anti-Aging and Esthetic Medicine, 22-25 Oct. 2009, Izmir, Turkey. Turkiye Klinikleri Journal of M edical Sciences. 2009;29(5(s1)):45.

\section{Book chapter}

Grech G, Scerri C, Scerri J, Cesuroglu T. Preventive and Predictive Genetics: A perspective. In: Grech G, Grossman I, editors. Preventive and Predictive Genetics: Towards Personalised Medicine. Advances in Predictive, Preventive and Personalised M edicine. 9: Springer International Publishing; 2015. p. 7-41.

\section{Health systems and policies, and health management related publications}

\section{Bookand bookchapters}

Savas BS, Cesuroglu T, editors. Sağlık Yöneticileri için Üreme Sağlı̆̆ı Hizmetleri Yönetimi [Management of Reproductive Health Services for Health Managers]: Birlesik Saglik Sistemleri Ltd. (United Health Systems Ltd.); 2004.

Cesuroglu T. Giriş - Üreme Sağlığında Sürekli Kalite Gelişimi [Introduction - Continuous Quality Improvement in Reproductive Health]. In: Savas BS, Cesuroglu T, editors. Sağlık Yöneticileri için Üreme Sağlığı Hizmetleri Yönetimi [Management of Reproductive Health Services for Health Managers]: Developed and published by BSS- United Health Systems Ltd. for United Nations Population Fund \& M inistry of Health of Turkey; 2004.

Cesuroglu T. Üreme Sağlığı Hizmetleri için Sürekli Kalite Gelişimi Uygulamaları ve Araçları [Continuous Quality Improvement Cycle Implications and Tools for Reproductive Health]. In: Savas BS, Cesuroglu T, editors. Sağıık Yöneticileri için Üreme Sağlığı Hizmetleri Yönetimi [Management of Reproductive Health Services for Health Managers]: Developed and published by BSS- United Health Systems Ltd. for United Nations Population Fund \& M inistry of Health of Turkey; 2004.

\section{Abstract}

Savas S, Cesuroglu T. An integrated approach to reach quality in health services: Clinical Work Flows. In: Kose A, Yarar O, eds. 2nd National Congress on Management of Health Care Institutions; 2004 March 04-07; Pamukkale, Denizli/Turkey. p 93-103. 


\section{Other publications}

\section{Article in scientific journal}

Aslan D, Altintas H, Emri S, Cesuroglu T, Kotan O, Koyuncu S, et al. Self-evaluations of tuberculosis patients about their illnesses at Ankara Ataturk Sanatorium Training and Research Hospital, Turkey. Respir M ed. 2004;98(7):626-31.

\section{Abstract}

Cesuroglu T, Ozvaris SB, Tureli D, Sancak O, Sozener U. Determination of Opinions and Contentment of Preclinical Students in Hacettepe University Faculty of M edicine. In: Abstract Book of the International Public Health Congress - "Health 21 in Action"; 2000 October 8-12; Istanbul, Turkey. p. 10.

\section{Contributions to Conferences and Symposia}

Cesuroglu T \& Savas S. 'Gentest Personalized Preventive Health Care Model' at Dutch Life Sciences Conference at Novio Tech Campus, 19 Nov. 2014, Nijmegen/ the Netherlands.

Cesuroglu T. 'Gentest Personalized Preventive Health Care Model' at the First Annual M eeting of the Directors of The Centers for Genetics Nutrition and Fitness for Health, 5 Nov. 2014, Costa Navarino/ Greece.

Cesuroglu T. "Implementation of Personalized Health Care in Health Systems" at Genetica Retreat organized by Maastricht University, 15 M ar. 2012, Rolduc/ the Netherlands.

Cesuroglu T. "Implementation of Personalized Health Care in Health Services in the Context of Public Health Genomics" at 1st World Conference of the European Association for Predictive, Preventive and Personalised Medicine (EPMA), 17 Sep. 2011, Bonn/ Germany.

Cesuroglu T. 'Public Health Genomics in Europe', in the mini-symposium on 'Public Health Genomics in the Netherlands', at Netherlands Public Health Congress, 6-7 Apr. 2011, Amsterdam/ the Netherlands.

Cesuroglu T. 'Perspectives in Personalized Health Care' at Post Academic Training Course on Youth Health, theme: Public Health Genomics, 25 Jan. 2011, Maastricht/ the Netherlands.

Cesuroglu T. 'Public Health Genomics and Reflections for Atherosclerosis' at the Symposium on Atherosclerosis and Its Complications in Clinical Practice, organized by Atherosclerosis Society of Turkey, International Atherosclerosis Society (IAS) and European Atherosclerosis Society (EAS), 11-13 Sep. 2009, Cesme/ Turkey.

Cesuroglu T. 'Public Health Genomics in Practice' at 'Public Health Genomics Symposium' at Dr. Andrija Stampar Institute of Public Health, 7 Jul. 2009, Zagreb/ Croatia. 
Cesuroglu T. 'Role of Nutrigenetics in Prevention, Risk Assessment and Management of Diabetes' at the $44^{\text {th }}$ National Diabetes Congress, 20-24 M ay 2009, Antalya/ Turkey.

Cesuroglu T. 'Chronic Complex Diseases and Genetics' at the Symposium on Public Health Genomics and Nutrigenetics, at the $3^{\text {rd }}$ International Congress on Molecular Medicine, 5-8 May 2009, Istanbul/ Turkey.

Cesuroglu T. 'OBESIS Project' at the $2^{\text {nd }}$ Istanbul International Symposium on Public Health Genomics - 'Chronic-Complex Diseases and Genetics', 26-27 Mar. 2009, Istanbul/ Turkey.

Cesuroglu T. 'Public Health Genomics, International Activities and a Practice Model for Personalized Health Care', at the $12^{\text {th }}$ World Public Health Congress, organized by World Federation of Public Health Associations, 1 M ay 2009, Istanbul/ Turkey.

Cesuroglu T. 'Molecular Genetics in Applications of Personalized Medicine', at 'Importance of Pharmacology in Clinical Applications Panels'; 5 Apr. 2006, Gaziantep University Faculty of Medicine, Gaziantep/Turkey, and 9 Jun. 2006, Cukurova University Faculty of M edicine, Adana/ Turkey. 
